\title{
FLUORESCENCE STUDIES OF AMINE-SUBSTITUTED AzaAnThracene Metal CompleXeS
}

\author{
Dissertation \\ zur Erlangung des mathematisch-naturwissenschaftlichen Doktorgrades \\ „Doctor rerum naturalium“ \\ der Georg-August-Universität Göttingen \\ im Promotionsprogramm Chemie \\ der Georg-August University School of Science (GAUSS)
}

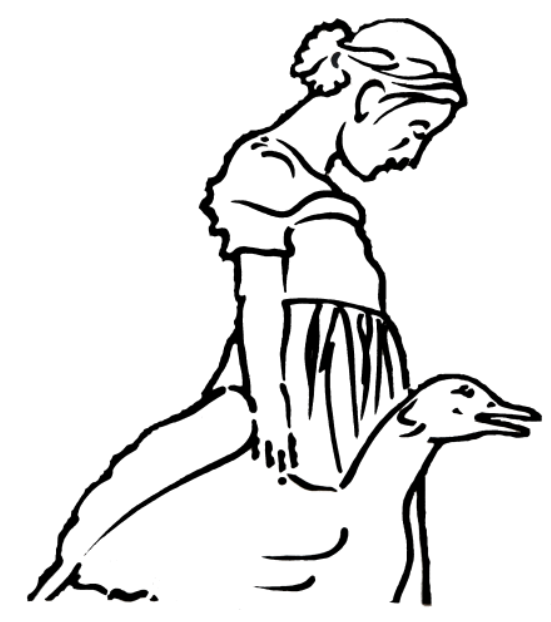

vorgelegt von Arne Visscher

aus Burgwedel

Göttingen, 2016 



\section{Betreuungsausschuss}

Prof. Dr. Dietmar Stalke, Institut für Anorganische Chemie

Prof. Dr. Franc Meyer, Institut für Anorganische Chemie

\section{Mitglieder der Prüfungskommission}

Referent: Prof. Dr. Dietmar Stalke

Korreferent: Prof. Dr. Franc Meyer

weitere Mitglieder der Prüfungskommission

Prof. Dr. Ricardo A. Mata, Institut für Physikalische Chemie

Prof. Dr. Konrad Koszinowski, Institut für Organische und Biomolekulare Chemie

Prof. Dr. Thomas Waitz, Institut für Anorganische Chemie

Dr. Michael John, Institut für Organische und Biomolekulare Chemie

Tag der mündlichen Prüfung: 17.06.2016 



\section{DANKSAGUNG}

Mein erster Dank gilt meinem Promotionsbetreuer Prof. Dr. Dietmar Stalke für die langjährige Unterstützung dieser Arbeit. Es war spannend, das im Arbeitskreis neue Thema der AcridinFluorophore frei erarbeiten $\mathrm{zu}$ dürfen und somit eine Alternative für das schon ausgiebig untersuchte Anthracen aufzeigen zu können.

Herrn Prof. Dr. Franc Meyer möchte ich für die freundliche Übernahme des Korreferats danken. Den weiteren Mitgliedern der Prüfungskommission danke ich ebenfalls für die Zeit, die sie sich genommen haben, meine Arbeit zu bewerten.

Meinen beiden langjährigen Labormitstreitern, David Dauer und Sebastian Wandtke, danke ich für die sehr angenehme Arbeitsatmosphäre und die vielen Gespräche auch außerhalb des Chemieuniversums. Dabei danke ich Sebastian für die immerwährenden Motivationsschübe, stets pünktlich im Labor zu erscheinen und David für die gegenseitige Unterstützung, die vorliegenden Ergebnisse auch endlich mal zu Papier zu bringen.

Meinen AC-F Praktikanten Grigory Shevchenko, Benjamin Gerdes, Michaela Bauer, Sayaka Shoji und Matheus Bukala danke ich für die synthetische Unterstützung im Labor.

Damit diese Dissertation verständlich und somit druckreif werden konnte, haben Lennard Krause, Thomas Niklas, Alexander Paesch und Timo Schillmöller ganze Arbeit geleistet. Vor allem Lennard und Thomas hatten scheinbar sehr viel Spaß den Verbrauch an roten Stiften bei Frau Gaugel zu erhöhen. Danke dafür!

Die Aufnahme von wirklich interessanten NMR Spektren war nur möglich, mit der fleißigen Hilfe von unserem NMR-Experten Sebastian Bachmann. Danke für die vielen abendlichen Messungen sowie auch für die Erklärung der Ergebnisse. Christoph Schnegelsberg danke ich für die superempfindlichen ESI-TOF Messungen meiner kaum löslichen Produkte. Bei Thomas Niklas bedanke ich mich für die wesentlich besseren ORCA-Rechnungen, so dass mir die Benutzung vom minütlich abstürzenden Avogadro weitestgehend erspart blieb.

Weiterhin bedanken möchte ich mich bei Thorsten Teuteberg und Ricardo Mata, die für mich sehr komplexe Rechnungen zu meinem „Super-Sensor“ durchgeführt haben. Deren Ergebnisse haben erheblich zum Verständnis der Acridin-Metall-Wechselwirkungen beigetragen. Erfreulicherweise hat Thorsten mir die Wechselwirkungen nicht nur anhand von Tabellen erklärt, sondern hat auch anschauliche Grafiken dazu angefertigt.

Was wäre unser Arbeitskreis ohne die vier durchweg laufenden Diffraktometer? Damit dies zumindest der Fall war, wenn meine Kristalle rotierten, möchte ich mich bei den fleißigen Maschinenschraubern bedanken. Besonders hervorzuheben sind dabei Felix Engelhardt und Lennard Krause, die zusammen mit Reent Michel auch meinen vielen kristallographischen Fragen standgehalten haben. Und wenn selbst deren Verfeinerungs-Knowhow irgendwann mal zu Ende ging, so konnte ich sicher sein, dass Regine Herbst-Irmer auch die kleinste Restelektronendichte noch zu verbessern wusste.

Dass ich diesem Arbeitskreis so lange treu geblieben bin, liegt unter anderem auch an der super Atmosphäre in dieser Gruppe. Immer wieder motivierend waren die vielen Kuchen mit noch mehr Kaffee, die sportlichen Einlagen am Kickertisch sowie auch das eine oder andere Feierabendbier 
in Peters Pinte. In diesen kurz gehaltenen Pausen konnte man überaus wichtige Diskussionen über interdisziplinäre Themen führen.

Während des langjährigen Studiums wurden aus Kommilitonen schnell gute Freunde. Danke für die gemeinsame Zeit, die wir in Göttingen verbracht haben. Ich hoffe, dass diese Freundschaften auch noch lange nach der Uni-Ära anhalten werden.

Einen besonderen Dank möchte ich meinen Eltern aussprechen, die mich immer in jeder Hinsicht unterstützt haben. Danke, dass ihr so seid, wie ihr seid.

Zuletzt, und daher von größtem Stellenwert, danke ich meiner Frau Isis-Helena. Ich danke dir für deine Geduld, deine Unterstützung und deine Liebe. Und dafür, dass du mich geheiratet hast. Der Titel Ehemann macht einen weitaus glücklicher als der, den ich durch diese Arbeit erhalten werde. 
Für meine Eltern

„Alle sagten: Das geht nicht.

Dann kam einer, der wusste das nicht und hat's gemacht. “

- o. $V$. 



\section{TABLE OF CONTENTS}

1. Introduction

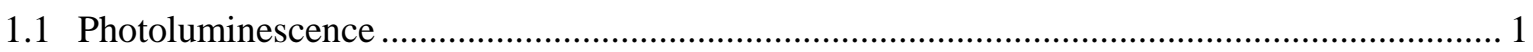

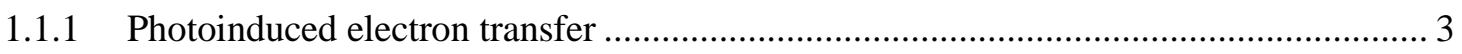

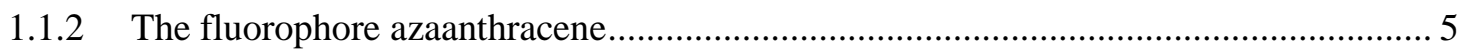

1.1.3 The detection of metal ions using acridine systems................................................. 9

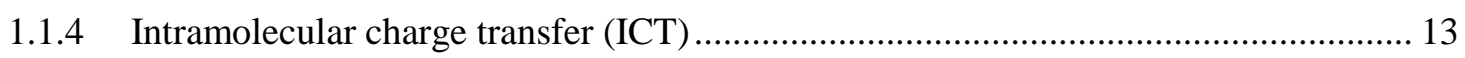

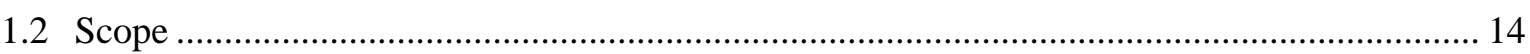

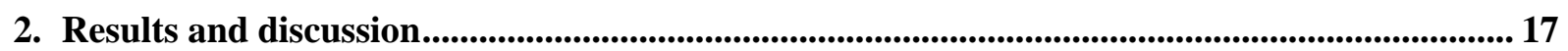

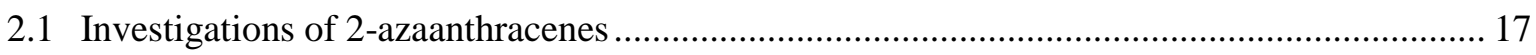

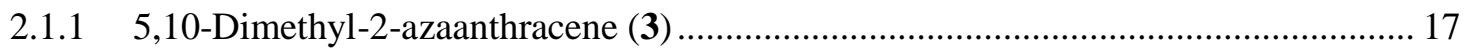

2.1.2 Investigations to the bromination of 5,10-dimethyl-2-azaanthracene (3)................... 19

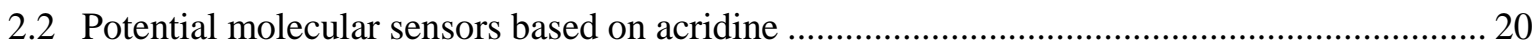

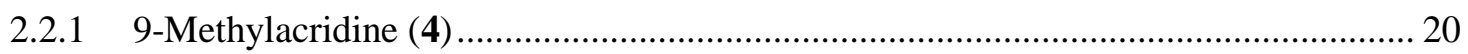

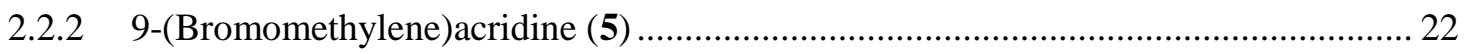

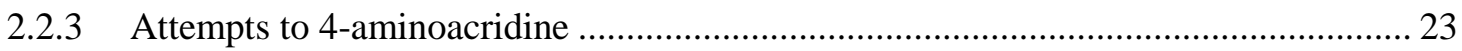

2.2.4 4,5-Bis(bromomethylene)acridine (8) and 4,5-bis(bromomethylene)-9-methyl-

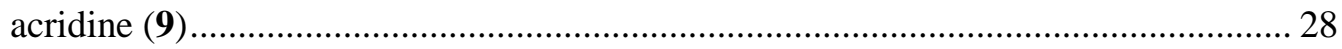

2.2.5 4,5-Bis(di(2'-methylthiobenzyl)aminemethylene)-9-methylacridine (10) ................... 32

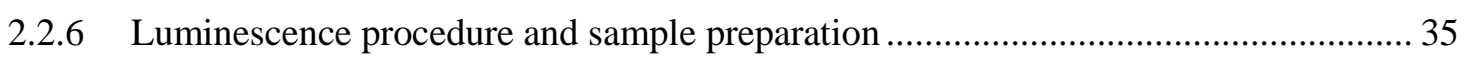

2.2.7 Luminescence properties of 4,5-bis(di(2'-methylthiobenzyl)aminemethylene)-

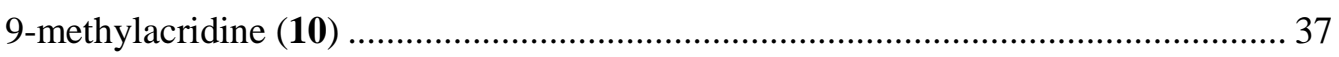

2.2.8 4,5-Bis $\left(N, N^{\prime}, N^{\prime}\right.$-trimethylethylenediaminemethylene)acridine (11) and its 9methyl derivative (12).

2.2.9 Luminescence properties of 4,5-bis $\left(N, N^{\prime}, N^{\prime}\right.$-trimethylethylenediamine-

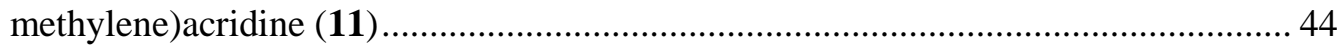

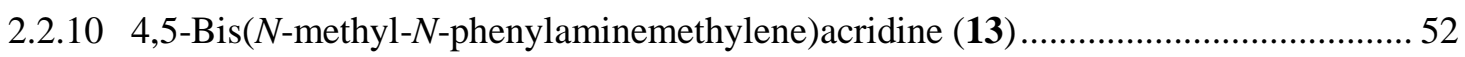

2.2.11 Luminescence properties of 4,5-bis( $N$-methyl- $N$-phenylaminemethylene)-

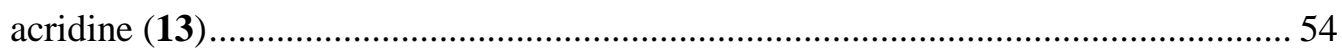

2.2.12 4,5-Bis $(N$-methyl- $N$-phenylaminemethylene)acridine hydrochloride (14) ................... 60

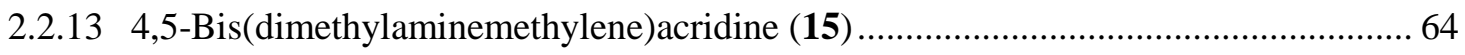

2.2.14 Luminescence properties of 4,5-bis(dimethylaminemethylene)acridine (15) ..............6 64

2.2.15 4,5-Bis(dimethylaminemethylene)acridine hydrochloride (16)................................ 74

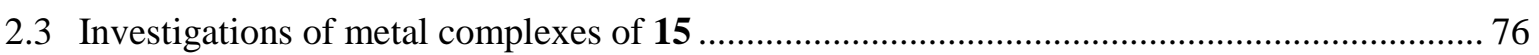

2.3.1 4,5-Bis(dimethylaminemethylene)acridine dibromido zinc(II) (17), dibromido cadmium(II) (19), and tetrakis( $\mu$-acetate) bis(acetate) zinc(II) (20) ........................... 76

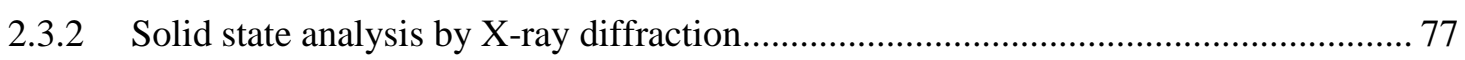

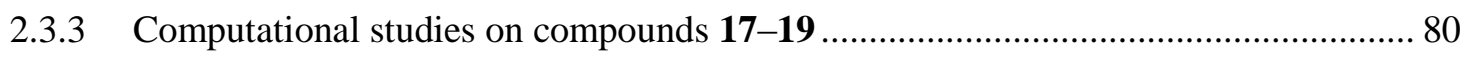

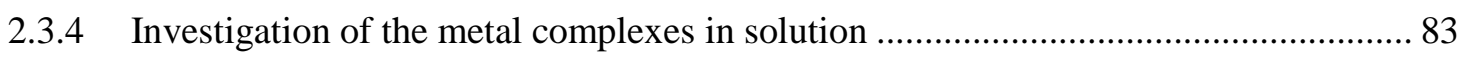




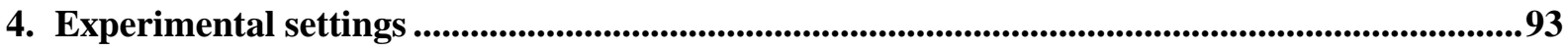

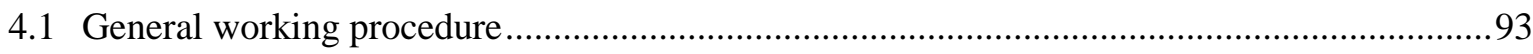

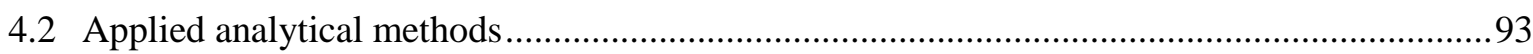

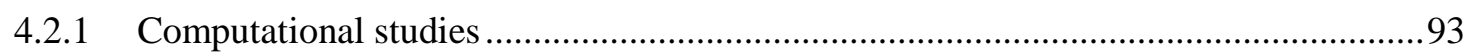

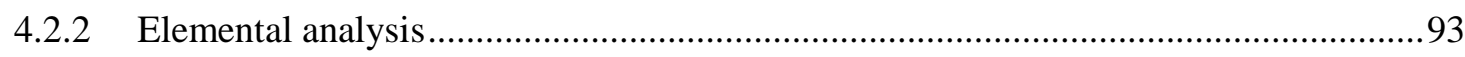

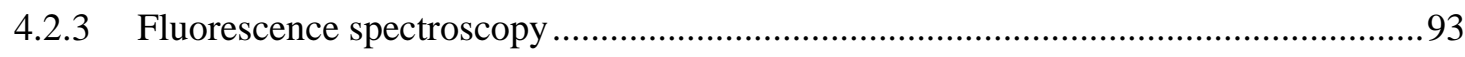

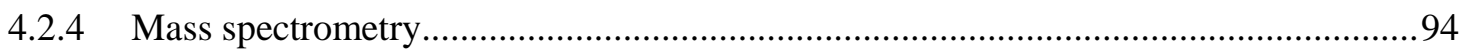

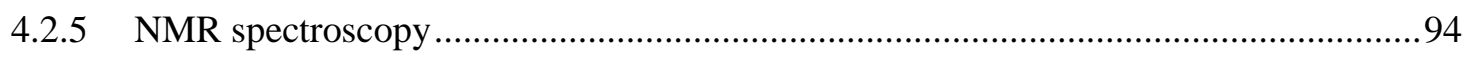

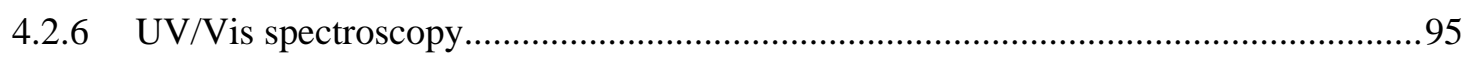

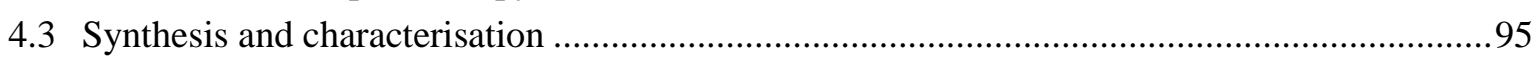

4.3.1 Synthesis of 1-phenoxycarbonyl-3-acetyl-4-(phenylethyl)-1,4-dihydro-

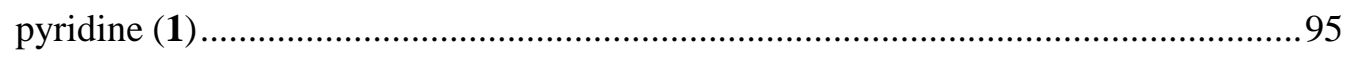

4.3.2 Synthesis of 3-acetyl-4-(phenylethyl)pyridine (2) …................................................96

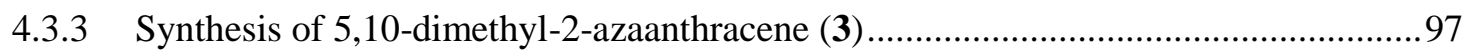

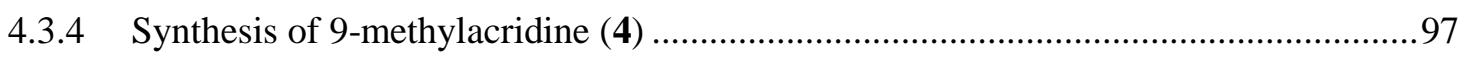

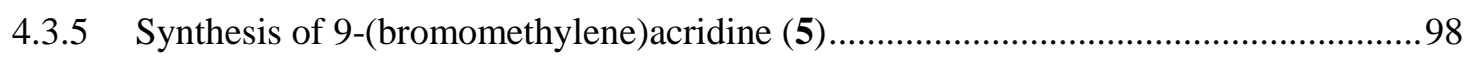

4.3.6 Synthesis of 2-bis( $p$-nitrobenzenesulfonyl)aminodiphenylamine (6) .........................99

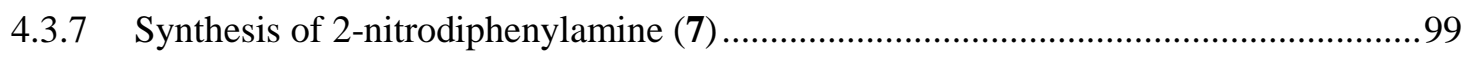

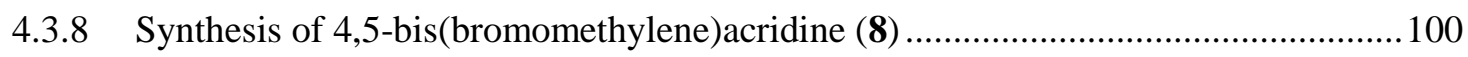

4.3.9 Synthesis of 4,5-bis(bromomethylene)-9-methylacridine (9) ................................... 101

4.3.10 Synthesis of 4,5-bis(di(2'-methylthiobenzyl)aminemethylene)-9-methyl-

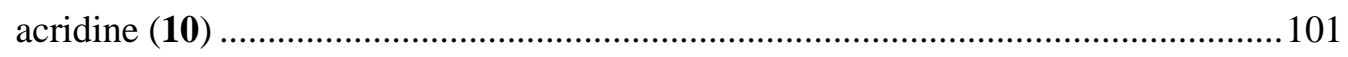

4.3.11 Synthesis of 4,5-bis $\left(N, N^{\prime}, N^{\prime}\right.$-trimethylethylenediaminemethylene)acridine

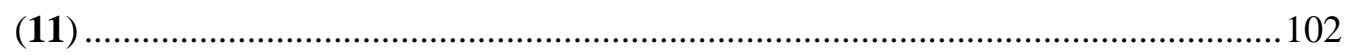

4.3.12 Synthesis of 4,5-bis $\left(N, N^{\prime}, N^{\prime}\right.$-trimethylethylenediaminemethylene)-9-methyl-

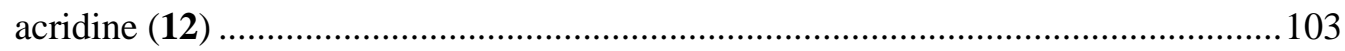

4.3.13 Synthesis of 4,5-bis $(N$-methyl- $N$-phenylaminemethylene)acridine (13).................... 104

4.3.14 Synthesis of 4,5-bis ( $N$-methyl- $N$-phenylaminemethylene)acridine

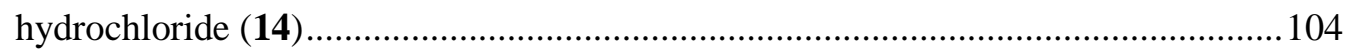

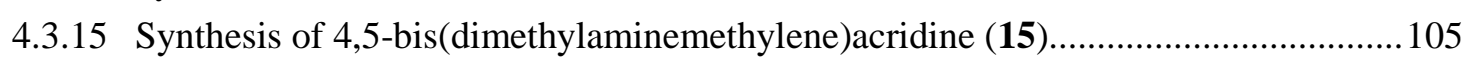

4.3.16 Synthesis of 4,5-bis(dimethylaminemethylene)acridine hydrochloride (16) ..............106

4.3.17 Synthesis of 4,5-bis(dimethylaminemethylene)acridine dibromido zinc(II) (17) ......107

4.3.18 Synthesis of 4,5-bis(dimethylaminemethylene)acridine dibromido

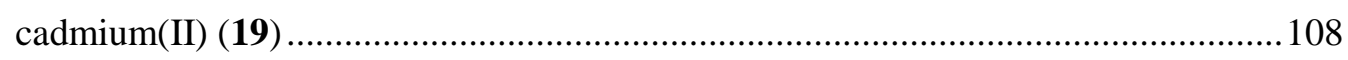

4.3.19 Synthesis of 4,5-bis(dimethylaminemethylene)acridine tetrakis( $\mu$-acetate)

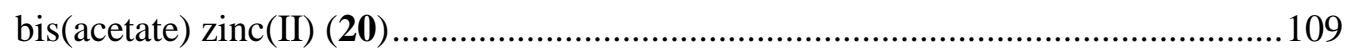

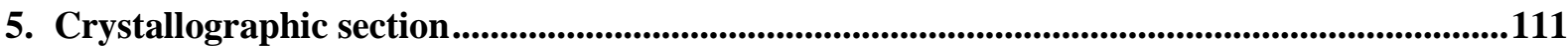

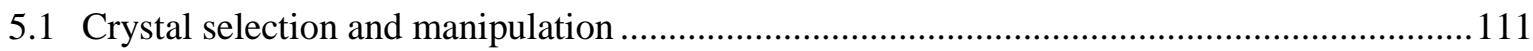

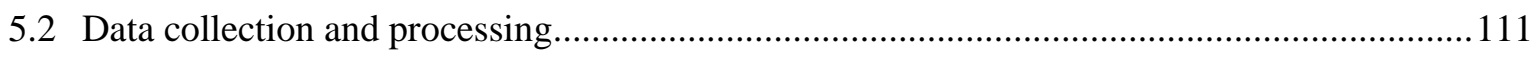

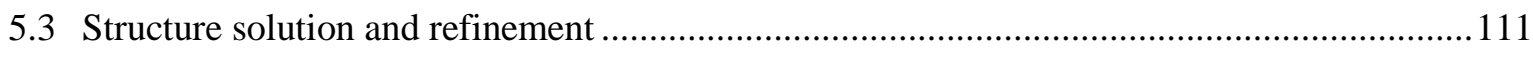

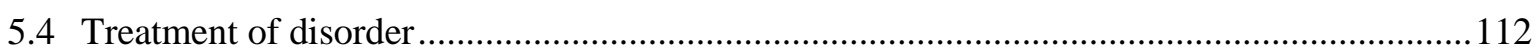

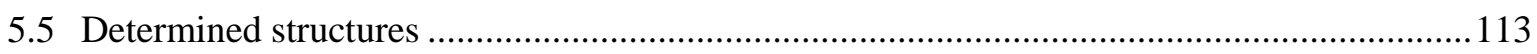

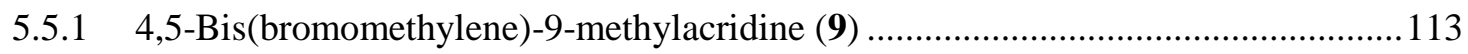


5.5.2 4,5-Bis(di(2'-methylthiobenzyl)aminemethylene)-9-methylacridine (10) ................. 114

5.5.3 4,5-Bis $(N$-methyl- $N$-phenylaminemethylene)acridine hydrochloride (14)................ 115

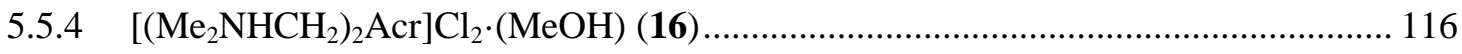

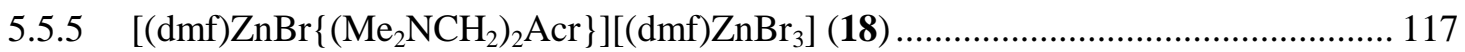

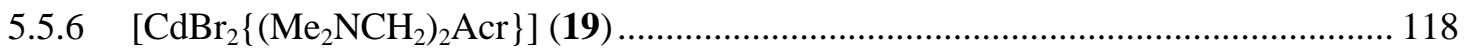

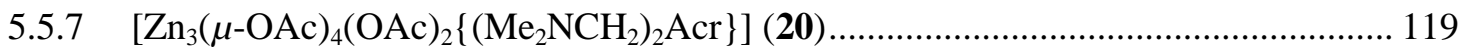

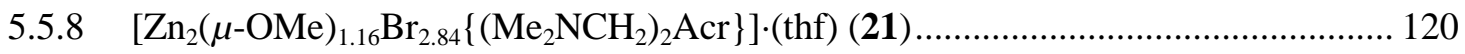

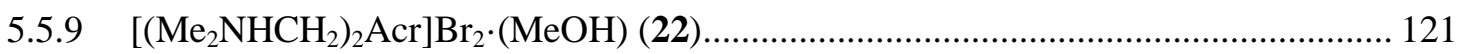

6. Crystal structure determination in collaborations........................................................................... 122

6.1 Structures determined for Mykyta Tretiakov (Prof. Dr. H. W. Roesky) ................................. 122

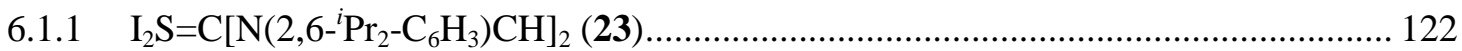

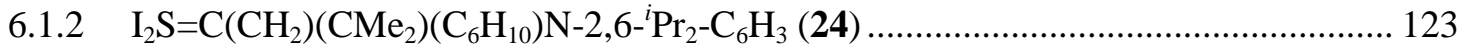

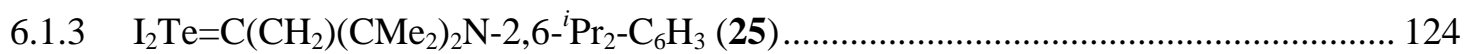

6.2 Structures determined for Bastian Milde (Prof. Dr. D. B. Werz) ……..................................... 125

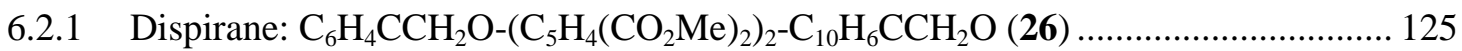

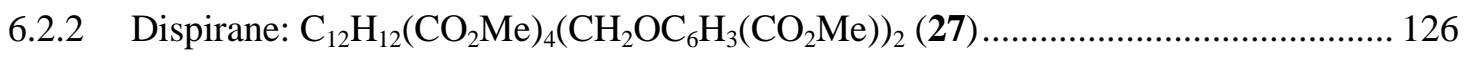

6.3 Structures determined for Tobias Schneider (Prof. Dr. D. B. Werz) ....................................... 127

6.3.1 Spiro[anthracene-9(10H), 1'(3' $H$ )-isobenzofuran]-3'10-dione (28) ............................. 127

6.3.2 10,15-Dihydro-5H-tribenzo[ $a, d, g]$ cyclononen-5-ol (29) ........................................ 128

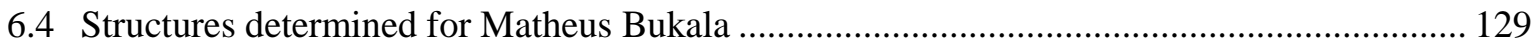

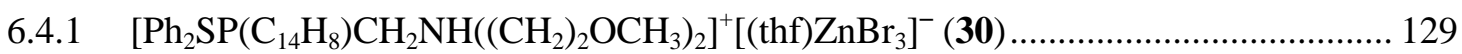

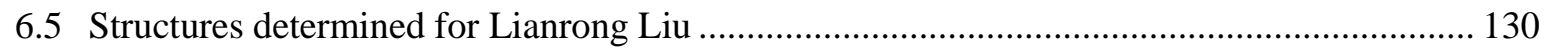

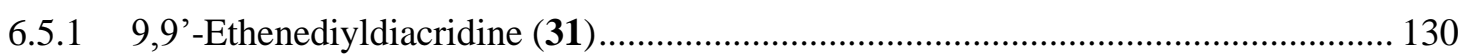

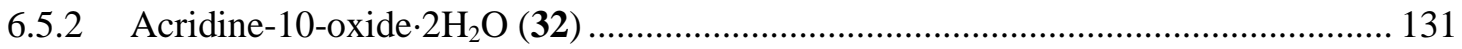

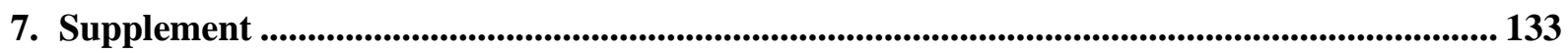

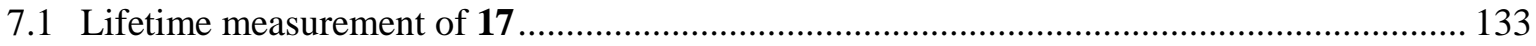

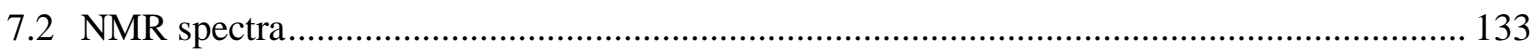

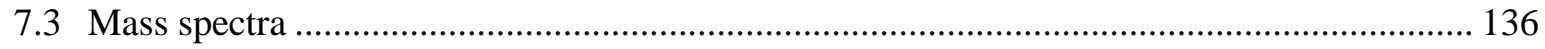

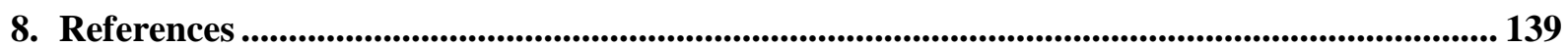




\section{ABBREVIATION INDEX}

\begin{tabular}{|c|c|c|c|}
\hline Acr & acridine & $\mathrm{Me}$ & methyl \\
\hline \multirow[t]{2}{*}{ BMME } & bromomethyl methyl & $\mathrm{MeCN}$ & acetonitrile \\
\hline & ether & $\mathrm{MeOH}$ & methanol \\
\hline conc. & concentrated & $\mathrm{MHz}$ & megahertz \\
\hline cps & counts per second & mmol & millimole \\
\hline DCM & dichloromethane & MS & mass spectrometry \\
\hline dem. & demineralised & NBS & $\mathrm{N}$-bromosuccinimide \\
\hline \multirow[t]{2}{*}{ DMEDA } & $N, N$-dimethylethylene- & NMR & nuclear magnetic \\
\hline & diamine & & resonance \\
\hline DMSO & dimethyl sulfoxide & $\mathrm{PE}$ & petroleum ether \\
\hline EA & ethyl acetate & PET & photoinduced electron \\
\hline EI & electron ionisation & & transfer \\
\hline eq. & equivalent(s) & $\mathrm{Ph}$ & phenyl \\
\hline ESI & electrospray ionisation & ppm & parts per million \\
\hline $\mathrm{Et}_{2} \mathrm{O}$ & diethyl ether & $R_{\mathrm{f}}$ & retardation factor \\
\hline \multirow[t]{3}{*}{ HEPES } & 4-(2-hydroxyethyl)-1- & $\mathrm{rt}$ & room temperature \\
\hline & piperazineethanesulfonic & sof & site occupation factor \\
\hline & acid & THF & tetrahydrofuran \\
\hline \multirow[t]{2}{*}{ HOMO } & highest occupied & TLC & thin layer \\
\hline & molecular orbital & & chromatography \\
\hline \multirow[t]{2}{*}{ HSAB } & hard and soft acids and & TOF & time-of-flight \\
\hline & bases & TrMEDA & $N, N, N^{\star}$-trimethyl- \\
\hline$I$ & intensity & & ethylenediamine \\
\hline \multirow[t]{3}{*}{ IUPAC } & International Union of & $\lambda$ & wavelength \\
\hline & Pure and Applied & $\lambda_{\mathrm{det}}$ & detected wavelength \\
\hline & Chemistry & $\lambda_{\text {exc }}$ & excitation wavelength \\
\hline \multirow[t]{2}{*}{ LUMO } & lowest unoccupied & & \\
\hline & molecular orbital & & \\
\hline M & molar & & \\
\hline $\mathrm{m} / \mathrm{z}$ & mass/charge ratio & & \\
\hline
\end{tabular}




\section{COMPOUND INDEX}<smiles>CC(=O)C1=CN(C(=O)Oc2ccccc2)C=C[C@H]1C(C)c1ccccc1</smiles>

1<smiles>CC(=O)c1cnccc1C(C)c1ccccc1</smiles>

2<smiles>Cc1c2ccccc2c(C)c2cnccc12</smiles>

3<smiles>Cc1c2ccccc2nc2ccccc12</smiles>

4<smiles>BrCc1c2ccccc2nc2ccccc12</smiles>

5<smiles>Cc1c2cccc(CBr)c2nc2c(CBr)cccc12</smiles>

9<smiles>Cc1c2cccc(CN(C)CCN(C)C)c2nc2c(CN(C)CCN(C)C)cccc12</smiles>

12<smiles>CN(C)Cc1cccc2cc3cccc(CN(C)C)c3nc12</smiles><smiles>O=S(=O)([O-])N(c1ccccc1Nc1ccccc1)S(=O)(=O)[O-]</smiles>

6<smiles>O=[N+]([O-])c1ccccc1Nc1ccccc1</smiles>

7<smiles>BrCc1cccc2cc3cccc(CBr)c3nc12</smiles>

8<smiles>CSc1ccccc1CN(Cc1ccccc1SC)Cc1cccc2c(C)c3cccc(CN(Cc4ccccc4SC)Cc4ccccc4SC)c3nc12</smiles>

10<smiles>CN(C)CCN(C)Cc1cccc2cc3cccc(CN(C)CCN(C)C)c3nc12</smiles>

11<smiles>CN(Cc1cccc2cc3cccc(CN(C)c4ccccc4)c3nc12)c1ccccc1</smiles>

13

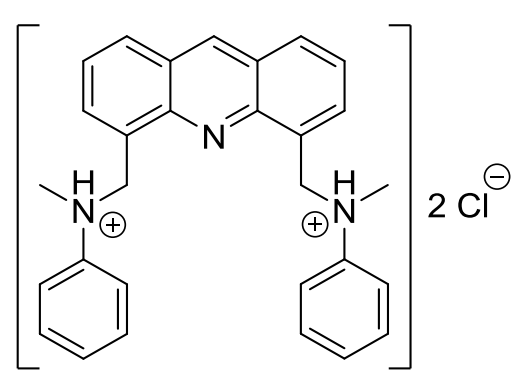

14<smiles>CN(C)Cc1cccc2cc3cccc(CN(C)C)c3nc12</smiles>

16<smiles></smiles>

17 


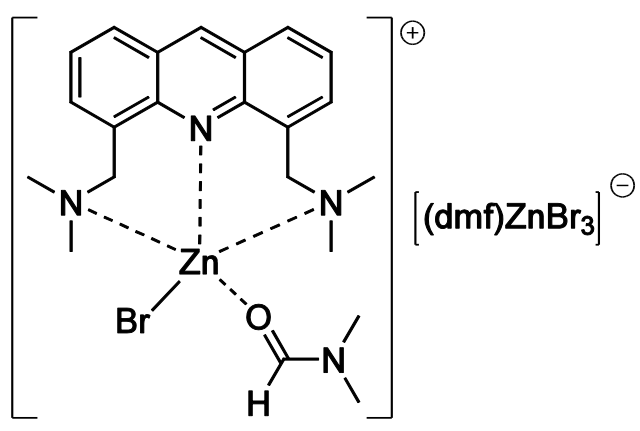

18<smiles></smiles>

19

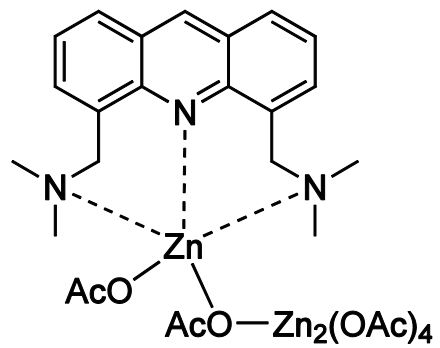

20 


\section{INTRODUCTION}

\subsection{Photoluminescence}

The existence of fluorescent and phosphorescent substances has always been a fascinating phenomenon, not only limited to scientists. Luminescent appearances have been reported centuries ago, without having any knowledge about the underlying processes. For instance, in 1565 the Spanish physician and botanist N. B. Monardes reported the peculiar blue colour from an infusion of a Mexican wood, later called Lignum nephriticum. ${ }^{[1]}$ He was surprised by the fact that the water was coloured bluish "though the wood is of white colour". ${ }^{[2]}$ In fact, the chemical evidence for the cause of this fluorescent phenomenon was only published in 2009. ${ }^{[3]}$ The pioneering physico-chemical investigations of important photoluminescence phenomina were made in the $19^{\text {th }}$ century, namely by E. Becquerel, D. Brewster, J. Herschel, and G. G. Stokes. Brewster and Herschel examined similar substances like chlorophyll and the historically famous quinine sulphate solution. ${ }^{[4]}$ Herschel observed that light may be scattered by particles or dispersed at the surface of solutions similar to prisms. He used the words 'epipolic dispersion' which is only a superficial effect, ${ }^{[4 a]}$ whereas Brewsters analysis refuted the surface explanation. On the basis of polarisation measurements, he introduced the 'internal dispersion' effect which "is a new property of light, produced by a peculiar action of certain solid and fluid bodies...".[4b] Both scientists made great discoveries in this new field but they did not figure out the difference in the wavelength of the incident and the resulting light.

At the same time, Stokes was also interested in the behaviour of quinine sulphate solutions. He established the term fluorescence in $1852^{[5]}$ when he noticed the longer wavelength of the light emitted by the irradiated solution. In further experiments, ${ }^{[6]}$ he used sunlight with a blue glass filter which selectively transmitted light below $400 \mathrm{~nm}$. This light was absorbed by the quinine solution causing the "beautiful celestial blue colour" ${ }^{\text {"[5] }}$. The crucial idea was to observe the solution through a glass filter only transmitting light above $400 \mathrm{~nm}$. Since he could still see the blue colour, he knew that the light emitted at a $90^{\circ}$ angle had to be of longer wavelength than the incident light. A schematic illustration of this experiment is depicted in Figure 1.1.

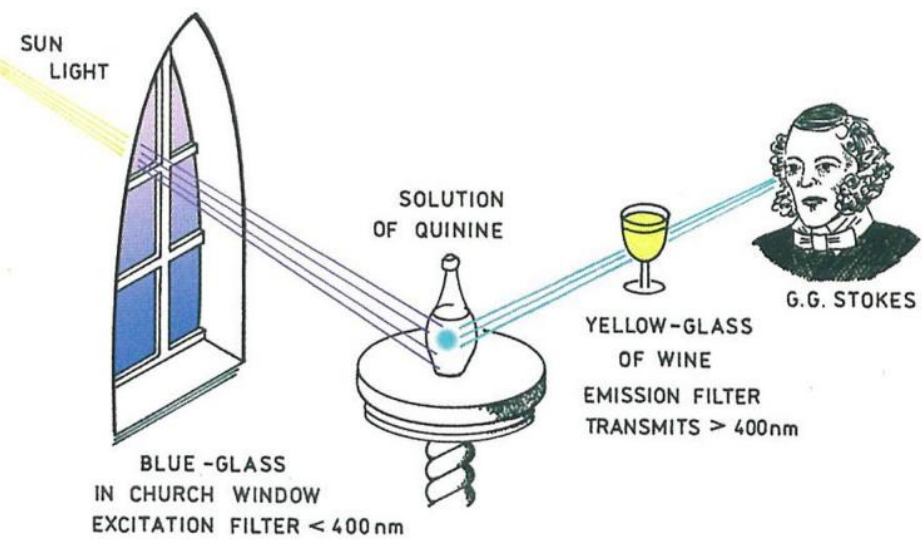

Figure 1.1: Schematic illustration of the experimental setup made by Stokes. ${ }^{[7]}$

It needs to be mentioned that Becquerel first stated this discrepancy in the adjusted and detected wavelength ten years before. He published his observations with calcium sulphide exposed to solar light beyond the violet part of the spectrum. ${ }^{[8]}$ He coined the term phosphorescence without 
making a difference between his observations and those of Stokes and stated that both are the same emission phenomenon only with different durations. It took some time until scientists like $A$. Jabloński contributed to our modern-day understanding of fluorescence and phosphorescence processes. ${ }^{[9]}$ These phenomena originate from different electronic states which is easily be demonstrated with the help of a (modified) Jablonski diagram (Figure 1.2).

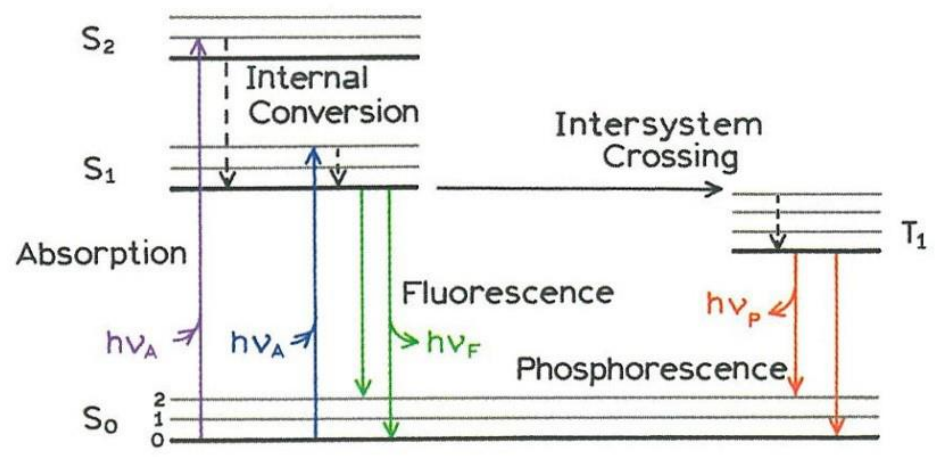

Figure 1.2: A possible illustration of a Jabłoński diagram demonstrating several spectroscopic processes. ${ }^{[7]}$

The diagram comprehensively describes the possible radiative and non-radiative transitions of an electronically excited sample. First of all, photons are absorbed by a chromophore in around $10^{-}$ ${ }^{15} \mathrm{~s}$ resulting in various electronic states $\left(\mathrm{S}_{1}, \mathrm{~S}_{2}, \ldots\right)$. Within these states different vibrational levels may be populated which are illustrated in the figure as thinner horizontal lines. Nevertheless, luminescence always occurs from the lowest vibrational level of $S_{1}$ (apart from some rare exceptions) since the excess energy is quickly dissipated (internal conversion). This is due to a strong overlap of the states along with a short relaxation time of about $10^{-12} \mathrm{~s}$. This nonradiative loss of energy explains the longer wavelength of the emission spectra, by now known as Stokes shift. According to this fact, the excitation wavelength does not influence the position of the emission spectrum as well as its shape. This is known as the Kasha rule. ${ }^{[10]}$

The difference between fluorescence and phosphorescence, which led to a discussion between Stokes and Becquerel, is also explained by the Jablonski diagram. Fluorescence occurs from the $\mathrm{S}_{1}$ state directly after the vibrational relaxation. It has a short lifetime of $10^{-6}-10^{-9} \mathrm{~s} .^{[11]}$ Electrons in the $S_{1}$ state can additionally undergo a forbidden spin conversion to the triplet state $T_{1}$ which is called intersystem crossing. The transition to the singlet ground state is also forbidden concerning the selection rules which leads to slow rate constants in comparison to the fluorescence. Therefore, the lifetime of phosphorescence lies in dimensions of $10^{-3}-10^{2} \mathrm{~s}^{[11]}$

Excitation and emission spectra can have well-structured curves if the energy distances of the vibrational levels are big enough for the resolution of the spectrometer. Furthermore, several features of luminescence spectra can only be explained by inspection of the anharmonic potentials illustrated in Figure 1.3. According to Boltzmann statistics, virtually only the lowest vibrational state is populated in the ground state at standard conditions. The molecule can be excited electronically to various vibrational level in $\mathrm{S}_{1}$. The Franck-Condon principle requires the transitions to be vertical since the movement of the core can be neglected in comparison to electron movement. ${ }^{[12]}$ 

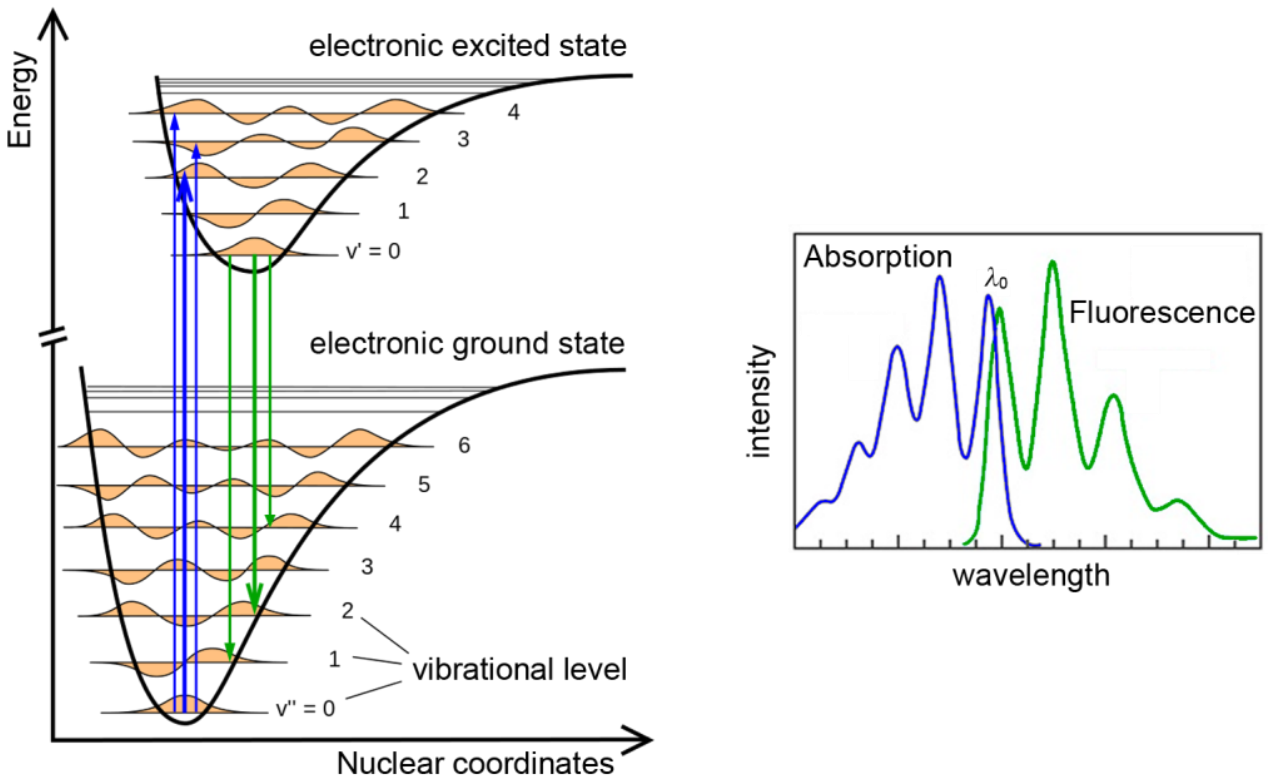

Figure 1.3: Illustration of the Franck-Condon principle and the origin of structured fluorescence spectra.

Additionally, transitions between vibrational states with a high orbital overlap are preferred. On the basis of this theory, the resulting spectra can have differently intensive band structures (Figure 1.3, right). Strong absorption due to high values of the wave function of both states results in more intense bands in the spectrum (e.g. $0 \rightarrow 2$, blue arrows). The same is true for the fluorescence emission marked in green.

The absorption of light by a fluorophore does not always imply the subsequent emission as luminescence occurrence. As already mentioned, the energy of molecules in higher electronic states is quickly dissipated by internal conversion to the lowest excited state $S_{1}$. Furthermore, different excited vibrational levels will quickly relax to the lowest level $\left(v^{\prime}=0\right)$. This is because the electronic configuration of a molecule changes upon excitation which leads to a fast reorganisation of the nuclei along with molecular vibration. The remaining energy can now be transformed to e.g. fluorescence occurrence or it is released in a non-radiative pathway due to collisions with other molecules. This is commonly present in concentrated solutions which is why fluorescence experiments are often realised with highly diluted samples. Hence, the analysed compound also collides with the solvent molecules. At ambient temperature, large compounds such as most dyes experience at least $10^{12}$ collisions per second. ${ }^{[13]}$

Luminescent compounds are nowadays applied in a broad range of industrial, pharmaceutical, and analytical products. ${ }^{[14]}$ The ongoing development of luminescent substances with tailored features like lifetimes and quantum yields is still a promising field of research with huge possibilities. ${ }^{[15]}$ One possibility is the use of these kind of molecules as sensory devices. Fluorescent materials can be designed to work as switches by turning their emission of light selectively on and off. ${ }^{[16]}$ As explained before, this process is so fast that it opens a variety of useful applications. In the following, the most established mechanism for fluorescence quenching will be discussed.

\subsubsection{Photoinduced electron transfer}

Sensor molecules should be able to switch instantaneously between a fluorescent on and off state or change their emission wavelength significantly upon addition of an analyte. To achieve this 
respond, several different mechanisms can be employed. Besides intramolecular charge transfer (ICT), ${ }^{[17]}$ metal-ligand charge transfer $(\mathrm{MLCT})^{[18]}$ or excimer formation, ${ }^{[19]}$ an extensively investigated concept is the photoinduced electron transfer (PET) ${ }^{[20]}$ effect. Here, a poorly or nonfluorescent ligand starts to emit light upon coordination of an analyte under UV irradiation. The quenching of the fluorophore is realised by its structural design (fluorophore-spacer-receptor) resulting in a very efficient electron transfer. This process is illustrated in Figure 1.4.

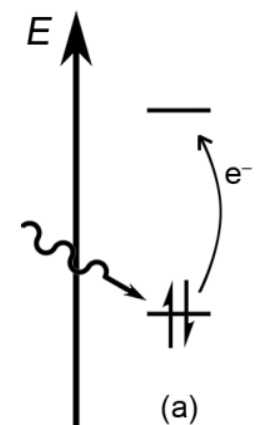

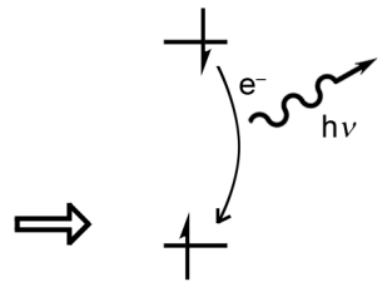

(b)

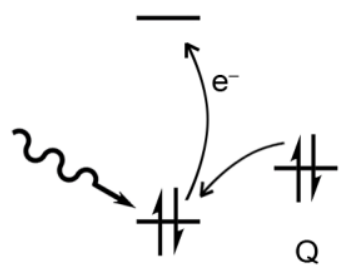

(c)

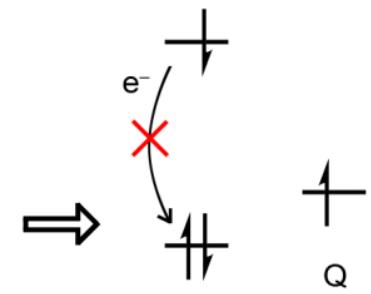

(d)

Figure 1.4: Mechanism of fluorescence $(a, b)$ and its quenching by photoinduced electron transfer $(c, d)$.

Usually, an electron in the ground state can be excited by irradiation of light (a). When it falls back to the ground state it loses its surplus energy and the phenomenon of fluorescence can occur (b). However, the excitation produces a vacancy in the ground state orbital, which may be filled with an electron from an orbital of a quencher (Q) which lies between these two states (c). In this case, no fluorescence emission can occur (d). The donating orbital can even belong to a separate molecule if it is in close proximity. ${ }^{[21]}$ In most cases it is a part of the fluorophore: here, especially amine moieties are chosen which are linked to the aromatic compound via a spacer unit. This spacer can be varied in its length, resulting in different efficiencies of the fluorescence quenching. ${ }^{[22]}$ Davidson and Trethewey have investigated this relation with derivatives of naphthylalkylamines. ${ }^{[23]}$ The results of this study are summarised in Table 1.1.

Table 1.1: Fluorescence quantum yields of different naphthylalkylamines. ${ }^{[23]}$ The derivatives with the most effective quenching properties are marked in blue.

\begin{tabular}{ccc|ccc}
\hline & & & & \\
\hline$n=1$ & 0.006 & $0.001(495 \mathrm{~nm})$ & $n=1$ & 0.018 & $0.006(495 \mathrm{~nm})$ \\
$n=2$ & 0.008 & $0.040(482 \mathrm{~nm})$ & $n=2$ & 0.003 & $0.028(470 \mathrm{~nm})$ \\
$n=3$ & 0.012 & $0.015(505 \mathrm{~nm})$ & $n=3$ & 0.010 & $0.010(470 \mathrm{~nm})$ \\
$n=4$ & 0.008 & $0.002(520 \mathrm{~nm})$ & $n=4$ & 0.012 & $0.012(500 \mathrm{~nm})$ \\
\hline \hline
\end{tabular}

In the left example, a spacer length of one methylene unit yields the highest quenching of the fluorophore whereas an ethylene bridge is more efficient for the right molecule. In literature, only one methylene unit is mainly used ${ }^{[24]}$ which is also true for the molecules prepared in the course for this thesis. Its implementation between the aromatic compound and the receptor allows the latter to rotate easily around its bonds. This provides the conformational freedom to interact 
electronically with the $\pi$-system of the fluorophore and results in an effective quenching of the fluorescence (Figure 1.5, a). At the same time the receptor is able to employ its lone pair in the complexation of analytes.

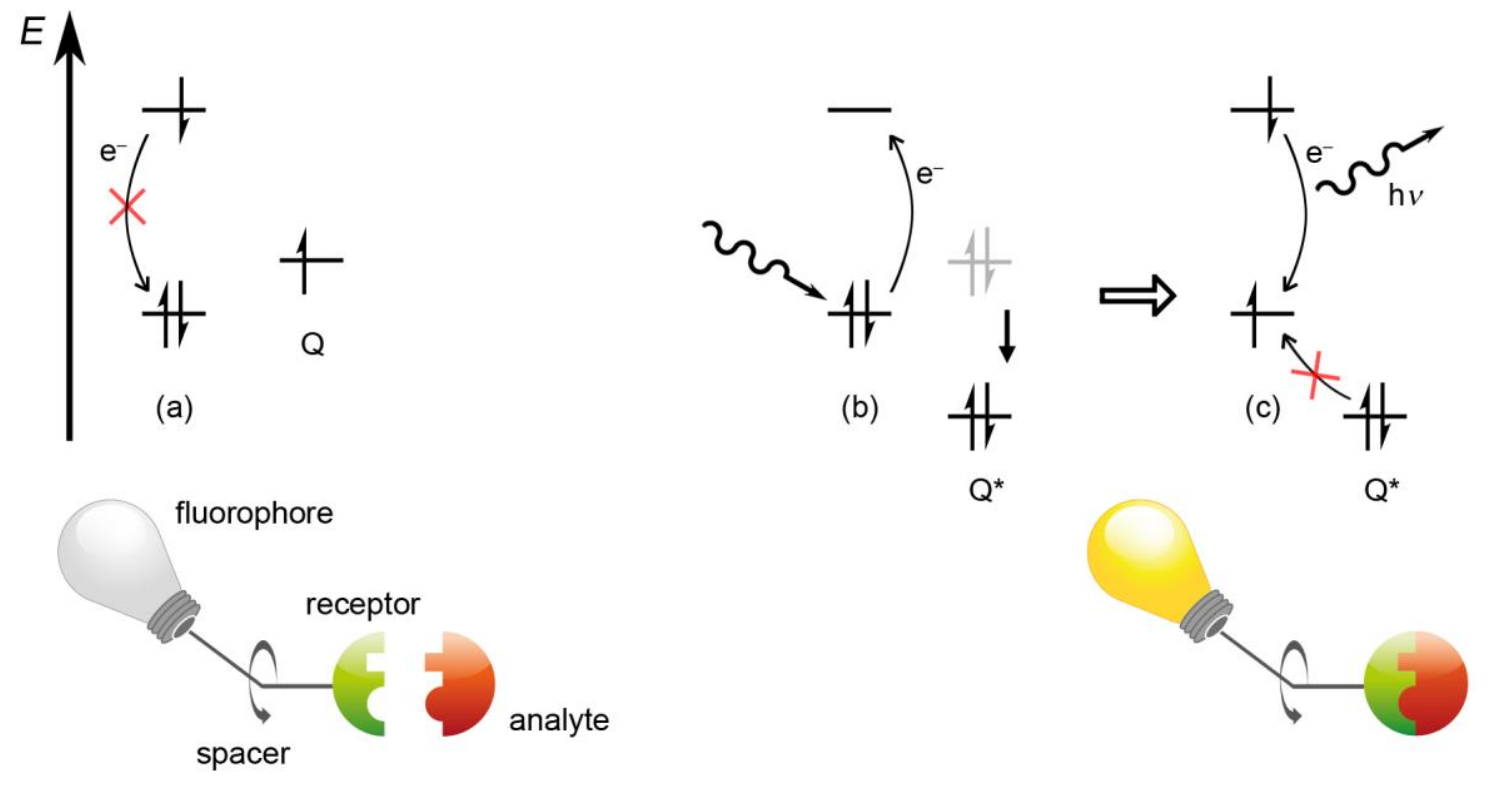

Figure 1.5: Left: Quenching of the fluorophore additionally illustrated by a scheme about the "fluorophorespacer-receptor" principle. Right: Inhibition of the PET through bonding of the quencher $(\mathrm{Q})$ to an analyte.

Through the binding of the quencher to e.g. a target metal ion, its redox potential is lowered resulting in an energetic state which lies underneath the energy of the excited fluorophore (b). If this is the case, the PET is hindered and the emission of light is facilitated (c). As already mentioned, amines are usually chosen for the role of the quencher/receptor. In order to donate an electron to the fluorophore, the amines need to have a higher potential energy than the aromatic compound.

\subsubsection{The fluorophore azaanthracene}

The choice of the fluorophore is not trivial since there is a variety of compounds described in the literature. Fluorophores mainly are polycyclic aromatic hydrocarbons with more or less extended $\pi$-systems. Anthracene is a very prominent example in this field, composed of three condensed benzene rings. Its derivatives and properties have also been investigated intensely by this working group. ${ }^{[25]}$ However, its congener azaanthracene, wherein one $\mathrm{CH}$ unit is substituted by a nitrogen atom, is far less investigated. Nevertheless, the usage of a nitrogen-containing aromatic system has the advantage of an additional donor function for the chelation of an analyte. This will be discussed exemplarily in the following chapter.
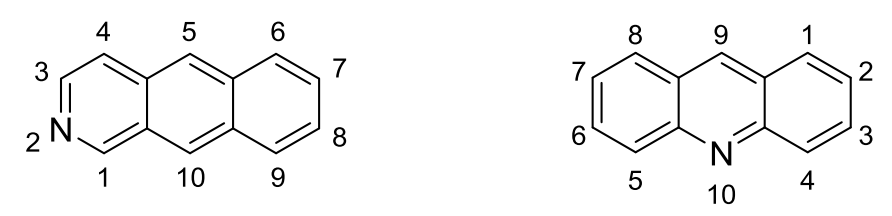

Scheme 1.1: Illustration of the Lewis diagrams of 2-azaanthracene (left) and 10-azaanthracene (right) including the correct IUPAC numbering of the atoms. 
The substitution can be realised in several positions, this thesis concentrates on the two derivatives 10-aza- and 2-azaanthracene (Scheme 1.1). One advantage of the latter compound is its low symmetry $\left(C_{\mathrm{S}}\right)$ in comparison to anthracene $\left(D_{2 \mathrm{~h}}\right)$ which leads to higher $\mathrm{S}_{0} \rightarrow \mathrm{S}_{1}$ transition rates and to an extent of spin-orbit coupling. In anthracene, the weak $\alpha$-band is forbidden while it is allowed and clearly resolved in 2-azaanthracene (Figure 1.6). As a result, the fluorescence quantum yield of the latter is higher than of its isoelectronic hydrocarbon (0.36 to 0.24 , respectively, measured in cyclohexane) ${ }^{[26]}$ However, it is a very rare representative of this group of fluorophores. A SciFinder ${ }^{\circledR}$ search of the term '2-azaanthracene' yielded only 71 hits, ${ }^{[27]}$ containing every year since its first synthesis in $1895^{[28]}$. A more expanded research with the words 'az(a)anthracene', and 'benz(o) $[g]$ isoquinoline' (IUPAC nomenclature), which includes further congeners, resulted in 140 hits. These low numbers of scientific publications concerning this compound was the motivation to synthesise derivatives of the fluorophore and to investigate its luminescence properties.

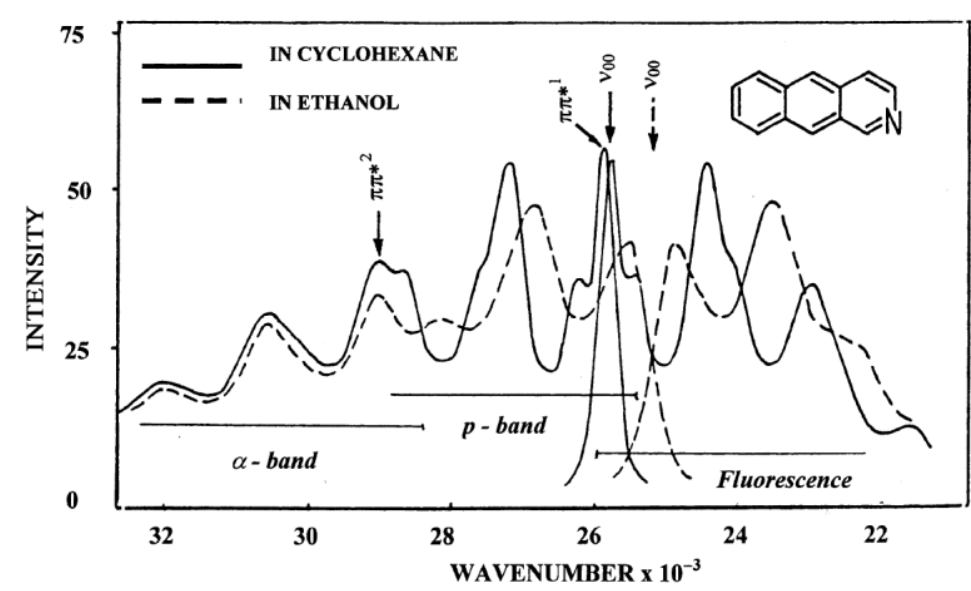<smiles>NCCNc1ccc(NCCN)c2c1C(=O)c1ccncc1C2=O</smiles>

Figure 1.6: Left: Absorption and fluorescence spectra of 2 -azaanthracene in different solvents. ${ }^{[26]}$ Right: Lewis diagram of the anti-cancer drug pixantrone.

Derivatives of the aromatic system are often used for DNA intercalation studies. Thereby, they may find application in the medicinal therapy of tumours. A well-known representative of these anti-cancer drugs is pixantrone which is used against aggressive lymphoma. ${ }^{[29]}$ It is based on the azaanthracene-5,10-dione structure and is depicted in Figure 1.6, right. Apart from the elaborate synthesis of this derivative, ${ }^{[30]}$ it could also be used as a possible molecular sensor. The ethylenediamine side arms in the 6- and 9-positions are good chelating receptors for e.g. cations. ${ }^{[31]}$ However, such derivatives have never been used for the fluorescence detection of metal ions according to the literature, apart from one publication. ${ }^{[32]}$ Here, the authors did brief investigations to possible metal ion interactions with the nitrogen atom of the fluorophore. Mainly, they focused on modern approaches to anthracenes and azaanthracenes by a $[2+2+2]$ cyclotrimerisation. Here, the synthesis for the latter compound is introduced. The authors conducted the cyclotrimerisation under $\mathrm{CpCo}(\mathrm{CO})_{2}$ catalysis in toluene using microwave irradiation $(300 \mathrm{~W})$, delivering the first intermediates in $80-94 \%$ yields. The following oxidation by DDQ resulted in six different substituted 2-azaanthracenes listed in Scheme 1.2. The reaction time of both steps is beneath half an hour involving good to excellent yields. A disadvantage is the usage of expensive starting materials and catalysts. 


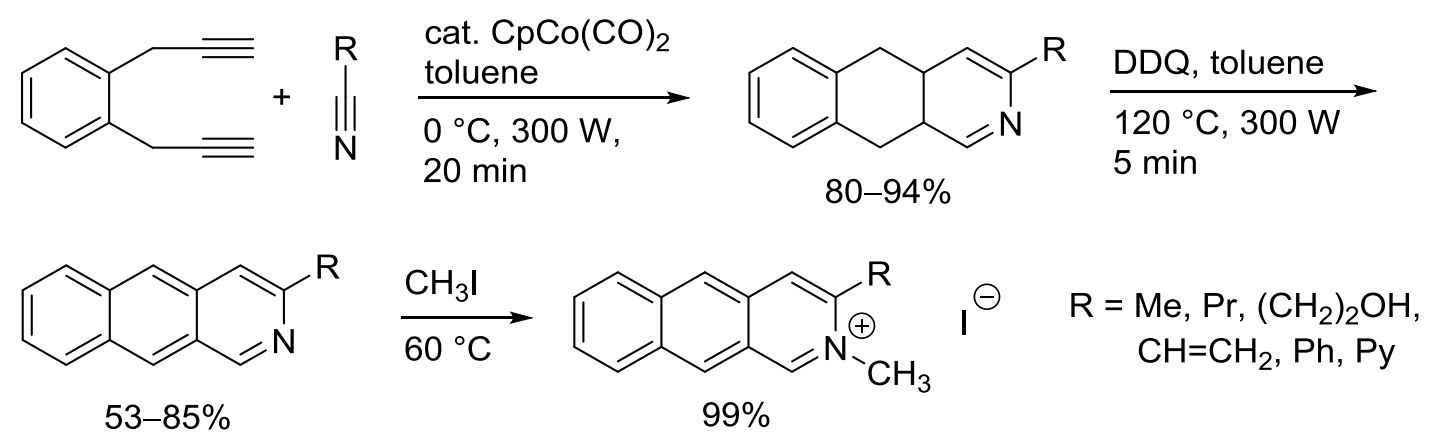

Scheme 1.2: Microwave assisted reaction pathway to 3-substituted 2-azaanthracene compounds. ${ }^{[32]}$
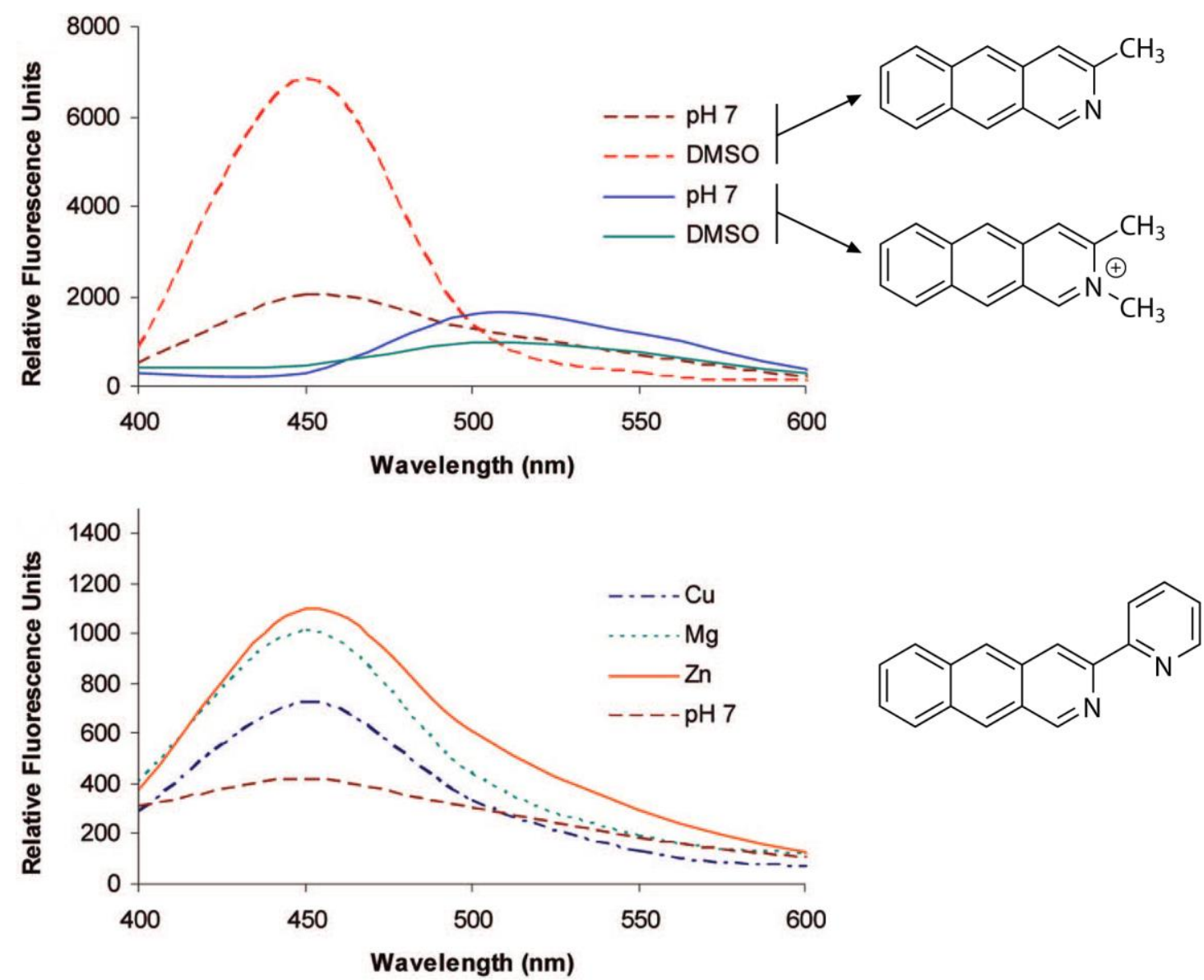

Figure 1.7: Emission spectra of different azaanthracene derivatives. ${ }^{[32]}$ Top: Comparison of mono- and dimethyl azaanthracene, demonstrating the effect of quaternisation on the fluorescence properties in DMSO and $\mathrm{H}_{2} \mathrm{O}$ ( $\mathrm{pH}$ 7); bottom: 3-pyridyl-2-azaanthracene in the presence of different divalent metal cations in aqueous solution.

Nevertheless, they introduced a fast and efficient approach to rare 2-azaanthracene derivatives. Additionally, they were able to methylate the nitrogen atom to induce a positive charge in the system which increases the solubility in an aqueous environment. Furthermore, the altering of the electronic properties of the fluorophore influences its emission spectrum investigated by the authors.

In the upper part of Figure 1.7, the emission spectra of 3-methyl-2-azaanthracene are shown, measured in protic $\left(\mathrm{H}_{2} \mathrm{O}\right.$ at $\left.\mathrm{pH} 7\right)$ and aprotic (DMSO) solvents. The difference in intensity can be explained by the ability of the nitrogen centre to undergo coordination to the solvent, mainly to its 
protons. Through the quaternisation of the nitrogen atom, a visible bathochromic shift in emission from blue to green was detected. This effect could also be generated in some cases by the use of dichloromethane as solvent. The use of a donating substituent closely associated to the nitrogen of the fluorophore (Figure 1.7, bottom) results in the coordination of some divalent metal cations $\left(\mathrm{Mg}^{2+}, \mathrm{Cu}^{2+}, \mathrm{Zn}^{2+}\right)$ increasing the emission intensity of the ligand. This small but very interesting effect was not further explained in the publication, but could be attributed to an intramolecular charge transfer (ICT, vide infra).

2-azaanthracene is commercially not available, and only a small number of further possible synthesis have been published in the last decades. All of them include at least three reaction steps with mainly small yields. In the following example, a dimethyl derivative is synthesised with a moderate yield of $29 \%$ (Scheme 1.3). ${ }^{[33]}$<smiles>CCCCOC(Cl)Cl</smiles><smiles>CCOC(C)C=NCc1cc(C)c2ccccc2c1C</smiles>

$65 \%$

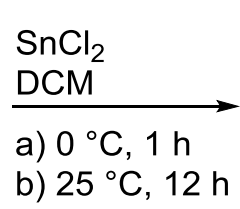<smiles>Cc1cc(C=O)c(C)c2ccccc12</smiles>

$73 \%$

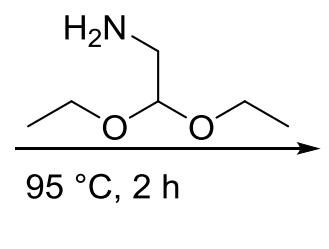

superphosphoric acid phosphorous pentoxide

a) $140{ }^{\circ} \mathrm{C}, 0.5 \mathrm{~h}$

b) $0{ }^{\circ} \mathrm{C}, 1 \mathrm{~h}$<smiles>Cc1c2ccccc2c(C)c2cnccc12</smiles>

$61 \%$

Scheme 1.3: Synthesis of 5,10-dimethyl-2-azaanthracene starting with a naphthalene derivative. ${ }^{[33]}$

Usually, pyridine derivatives are chosen as starting materials to introduce the heteroaromatic ring. ${ }^{[34]}$ In this case, the authors are using 1,4-dimethyl naphthalene which is substituted in the 2position. The synthesis involves three steps with acceptable yields. The reaction times are mainly short and the reagents are affordable. This is an important factor since the azaanthracene product is only the starting material for further reactions towards possible sensor systems. Therefore, the two methyl groups at the 5- and 10-positions are another advantage of this pathway since they should easily undergo substitution reactions with nucleophiles. This issue is again discussed in Chapter 2.1.1.

10-azaanthracene was discovered in 1870 by Graebe and Caro who found an unknown compound with basic properties when actually isolating anthracene out of coal tar. ${ }^{[35]}$ They gave it the name acridine due to its acridly effect to the skin. This name is still common today, apart from the systematic IUPAC name dibenzo[ $b, e]$ pyridine. Likewise, one of its first synthesis thirteen years later by Bernthsen ${ }^{[36]}$ is also still applied in current literature ${ }^{[37]}$ as well as in this thesis (Scheme 1.4). Acridine derivatives are mainly established in the field of bioorganic chemistry. Here, they are used as fluorescence dye for e.g. DNA marker. ${ }^{[38]}$ In the medicinal area, several derivatives are applied as anti-malaria drug ${ }^{[39]}$ or even as a "promising candidate for the treatment of aggressive, chemoresistant cancer". ${ }^{[40]}$ 


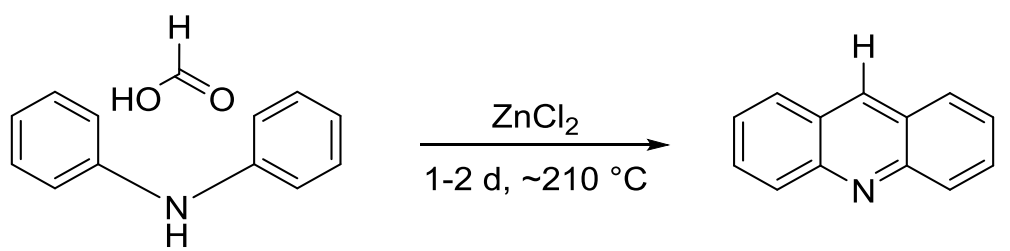

Scheme 1.4: Reaction of diphenylamine with formic acid yielding acridine according to the procedure of Bernthsen. ${ }^{[36]}$

However, the idea of using azaanthracenes as chemical sensors has mainly been pursued in the last decade. A SciFinder ${ }^{\circledR}$ search with the keywords 'acridine' and 'sensor' from 1870-2015 yielded 117 hits. ${ }^{[27]} 70$ entries thereof have been published only in the period from 2010-2015. For comparison, the use of the single term 'acridine' results in 29,100 hits.

One possible reason for the increased interest is the publication of Galy and Chiron in 2003. ${ }^{[41]}$ They introduced the highly useful precursor $\mathbf{8}$ for an easy and straightforward synthesis of acridinyl amines utilising the "fluorophore-spacer-receptor" design (Scheme 1.5).

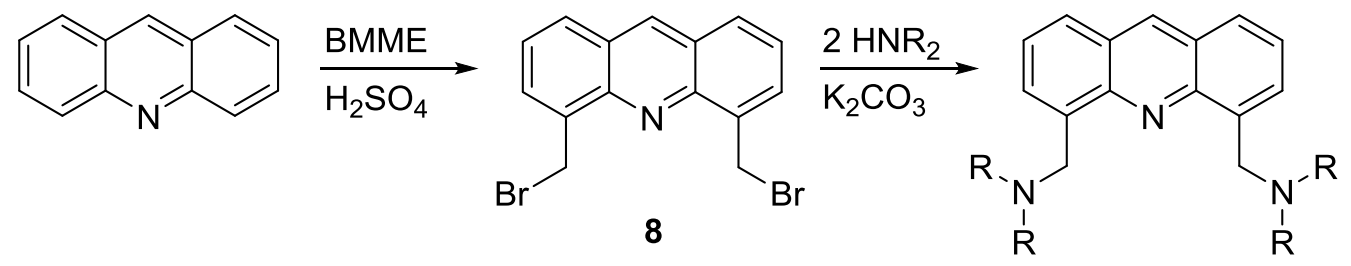

Scheme 1.5: Brief illustration of the synthetic route to potential molecular sensors based on acridine. The precursor 8 was implemented $2003^{[41]}$ and since then is used by numerous researchers.

The crucial reagent in this synthesis is the bromomethyl methyl ether (BMME) which allows to introduce two bromomethylene units to the aromatic system. This is carried out in a single step which is a tremendous advantage over further synthesis routes published only two years before. ${ }^{[42]}$ Here, the authors needed five steps to obtain the mono-bromomethylated species of acridine. The synthesis depicted in Scheme 1.5 will be explained in detail in Chapter 2.2.4.

\subsubsection{The detection of metal ions using acridine systems}

The detection of metal ions in solution has always been an important topic in analytical chemistry, environmental protection and medicinal applications. Metal ions like magnesium, calcium, or zinc are essential components of many enzymes in the human body. ${ }^{[43]}$ Especially $\mathrm{Zn}^{2+}$ plays a fundamental role in many different areas; e.g. in the emergence of Alzheimer's disease. ${ }^{[44]}$ Moreover, its level of concentration could help to diagnose the growing of tumour cells in the prostate. ${ }^{[45]}$ Zinc deficiency increases the susceptibility to a variety of pathogens and is of central importance for the immune system. ${ }^{[46]}$ However, zinc ions are spectroscopically silent which makes it difficult to detect them by typical analytical methods like NMR spectroscopy. The heavier homologue $\mathrm{Cd}^{2+}$ is known to be a very toxic metal ion. Over several decades in the first half of the $20^{\text {th }}$ century, hundreds of people in Japan were affected by the deadly itai-itai disease due to a cadmium polluted river. ${ }^{[47]}$ Therefore, it is vital to have a fast working and very sensitive method for the detection of metal ions in e.g. blood or water supplies. Fluorescence spectroscopy combined with suitable fluorescent sensors is a well-recognised method for such analysis. According to this, a multitude of publications is present in the literature, today. ${ }^{[15,25 a, 48]}$ However, 
acridine based sensors for cationic analytes are less commonly used, although its number of publications is rising significantly in the last few years as mentioned before.

The first example introduced in this chapter (Figure 1.8) has been selected due to the comparison of the fluorophores anthracene and 10-azaanthracene - both equipped with the same receptor. Furthermore, Yoon et al. stated, "any acridine derivative bearing ligand on the 4,5-position via methylene linkage has not [yet] been studied as fluorescent chemosensors for metal ions.". [49] They tested several metal ions in an aqueous buffer solution whereby selectivity towards zinc ions was detected.
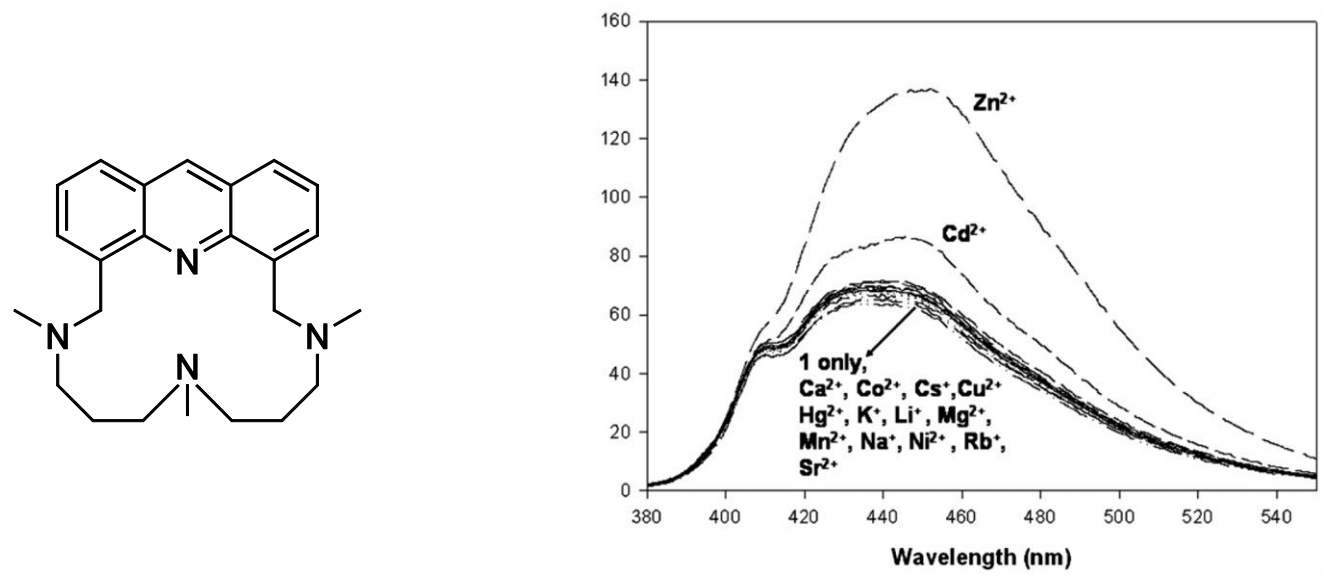

Figure 1.8: Molecular sensor for $\mathrm{Zn}^{2+}$ ions in an aqueous buffer solution. ${ }^{[49]}$

Interestingly, only the acridine based compound showed fluorescence response to $\mathrm{Zn}^{2+}$. The authors consider a binding contribution of the acridine nitrogen atom to the zinc ion due to these results. Nevertheless, they did not have any structural evidence for this hypothesis and the sensitivity of the sensor system is not very high. However, this publication confirms the application demand of acridine as a useful fluorescent part of sensor systems.

One year later, the same working group published another 4,5-substituted acridine derivative working as a chemical sensor. ${ }^{[50]}$ The published compound has the double number of donor sides, using two dipicolylamines as receptor units. In this case, they did not use the amines for the detection of metal ions like in the publication before, but for the preparation of a two-fold zinc complex (Figure 1.9, A).

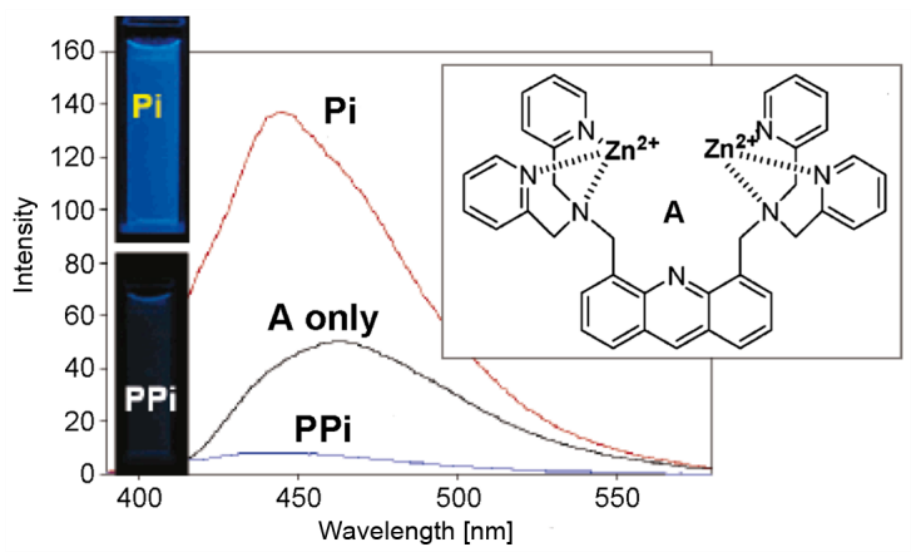

Figure 1.9: Fluorescence spectra of a Pi and PPi sensor, using a 4,5-bis(dipicolylaminemethylene)acridine which coordinates two zinc ions. ${ }^{[50]}$ 
Each dipicolylamine coordinates a zinc ion involving three nitrogen atoms. The rest of the coordination sphere is filled with $\mathrm{NO}_{3}{ }^{-}$anions. This complex is now acting as a detector for pyrophosphate (PPi) and inorganic phosphate $(\mathrm{Pi})$ anions which play important roles in signal transduction and energy storage in biological systems. ${ }^{[51]}$ Other anions like $\mathrm{HSO}^{-}, \mathrm{CH} 3 \mathrm{COO}^{-}$, and the whole group of halides do not alter the fluorescence spectrum significantly. The remarkable feature of this sensor system is the availability of two different mechanisms signalling the presence of Pi with a CHEF (chelation enhanced fluorescence) effect and the presence of PPi with a selective CHEQ (chelation enhanced fluorescence quenching) effect. The reduction of the fluorescence by the addition of a zinc complexing anion like PPi is easily comprehensible. When the metal ion is no longer bonded to the nitrogen atoms of the sensor, the amines act as quenchers due to the discussed PET effect. The CHEF mechanism is more complicated in this case. The authors state, that the effect can be attributed to additional hydrogen bonding between the hydrogen of the $\mathrm{OH}$ group in the $\mathrm{Pi}$ anion and the nitrogen atom of acridine. This correlation has been made by other authors as well. ${ }^{[52]}$ Additionally, they refer to the fluorescence enhancement observed by the coordination of zinc ions with the acridine unit, disucussed before in Figure 1.8. Especially in this case, a structural proof would be a great advantage. However, they could show the affinity of the quencher side arms to the zinc ion by X-ray diffraction which is a rare exception in literature.

Besides the usage of amine groups for the receptor side arms of acridine, oxygen donors can be used, too. Huszthy et al. have synthesised acridone and acridine derivatives bearing 18-crown-6 ethers in the 4,5-position. With these compounds they are able to detect optically active salts (e.g. $\alpha$-phenylethylamine hydrogen perchlorate ${ }^{[53]}$ and also to separate these enantiomers by the use of stationary acridone phases ${ }^{[54]}$. Some of their derivatives work as fluorescence sensors for $\mathrm{Cu}^{2+}$ and $\mathrm{Pb}^{2+}$ ions ${ }^{[55]}$ or for $\mathrm{Ca}^{2+}$ and $\mathrm{Zn}^{2+}$ ions ${ }^{[56]}$. The latter will be presented here with the focus on the different fluorescent backbones.
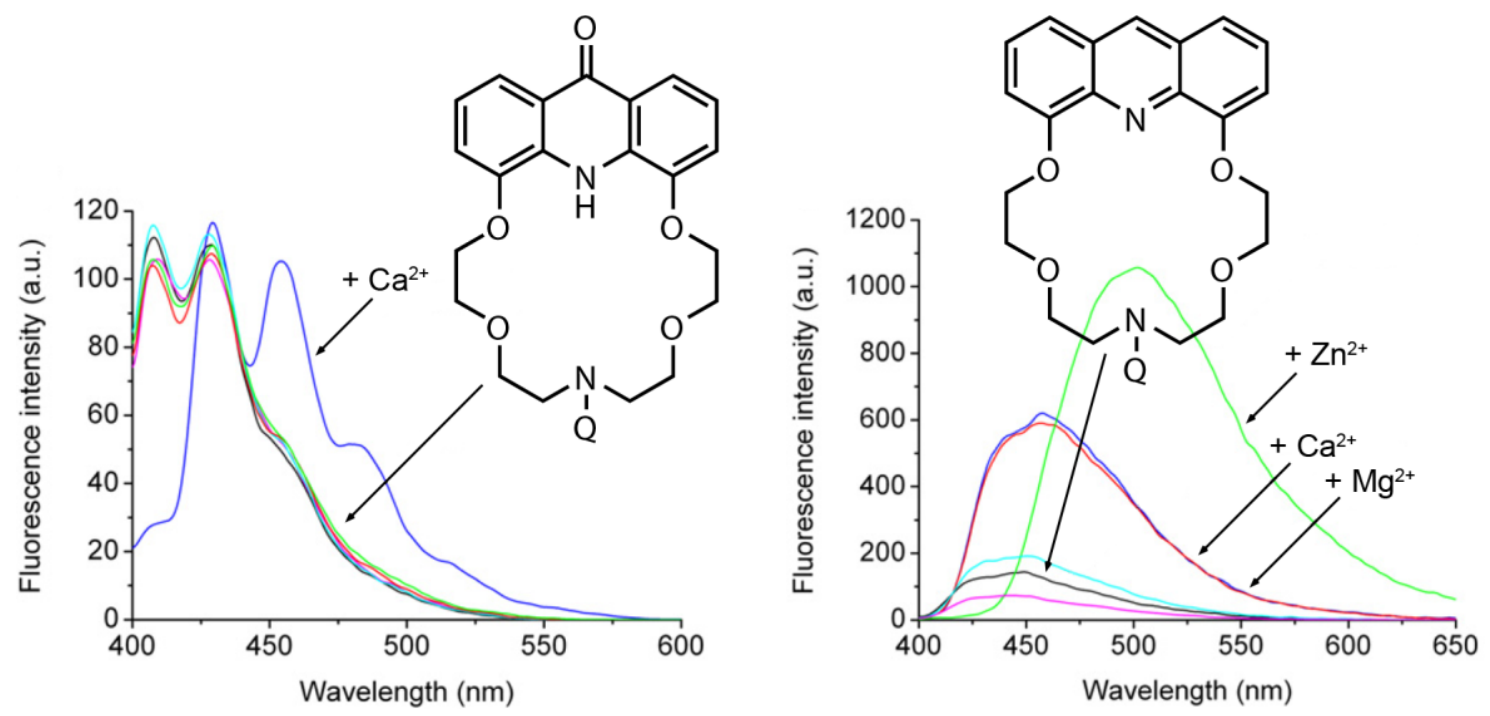

Figure 1.10: Emission spectra of 18-crown-6 ether derivatives based on acridone (left) and acridine (right) with $\mathrm{Q}=$ tetramethyl pyrroline. ${ }^{[56]}$ Spectral changes were shown upon addition of metal ions (1000 eq.) in MeCN: $\mathrm{Na}^{+}$: cyan, $\mathrm{K}^{+}$: magenta, $\mathrm{Mg}^{2+}$ : red, $\mathrm{Ca}^{2+}$ : blue, $\mathrm{Zn}^{2+}$ : green).

The coordination of metal ions is realised by an 18-crown-6 ether in both cases containing four oxygen and two nitrogen atoms as donor sites. However, they generate different results in the 
selectivity of the analytes by the use of acridine on the one hand and the oxidised species acridone on the other hand. The acridone compound is only sensitive towards $\mathrm{Ca}^{2+}$ ions, signalled with a bathochromic shift of $20 \mathrm{~nm}$ but without any change in intensity (Figure 1.10, left). The reduced species responds to $\mathrm{Ca}^{2+}, \mathrm{Mg}^{2+}$, and $\mathrm{Zn}^{2+}$ ions. The two earth alkali metals influence the spectrum in the same manner with a significant increase of the intensity (Figure 1.10, right). With the addition of zinc ions, the intensity rises stronger and an additional appreciable bathochromic shift of $50 \mathrm{~nm}$ is observed. The authors only describe their results without any interpretation of the spectra. They did not provide further analysis of the complexes to get an idea about the coordination behaviour and the possible influence of the metal ions to the fluorophore. Nevertheless, an interaction of $\mathrm{Ca}^{2+}$ ions, respectively $\mathrm{Zn}^{2+}$ ions, with the $\pi$-system is probable since the shift of the emission wavelength can be attributed to changes within the electronic state of the aromatic systems.

The demand for structural insights into the ligand-metal complexes determined by fluorescence spectroscopy is fulfilled by the next example. However, this is done with a time lag of five years and by two different research groups. In the first place, the ligand 4,5-bis $(N, N$-di $(2-$ hydroxyethyl)iminomethyl)acridine (BHIA) was synthesised 2011 by Jin et al. ${ }^{[57]}$ using the wellestablished route utilising BMME. The advantage of the published compound is the use of diethanolamines as they guarantee sufficient water solubility in combination with the binding ability to selected metal ions.

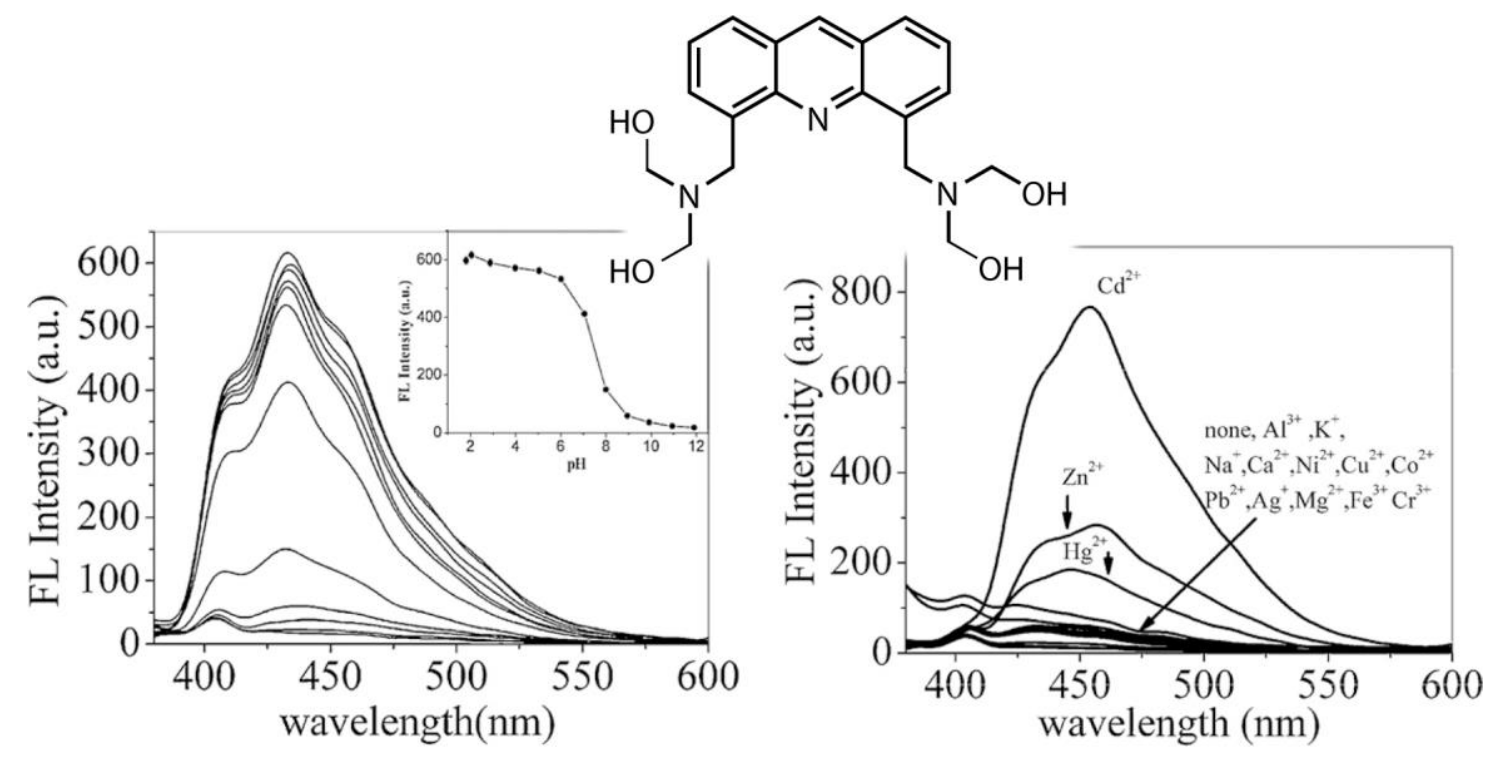

Figure 1.11: Emission spectra of the BHIA compound of Jin et al. ${ }^{[57]}$ showing the pH dependency in water (left) and the selectivity to $\mathrm{Cd}^{2+}$ ions in HEPES buffer with $\mathrm{pH} 7.4$ (right).

The potential sensor compound shows a great dependency on the $\mathrm{pH}$ value of the aqueous solution (Figure 1.11, left). With decreasing $\mathrm{pH}$ the emission intensity rises due to the protonation of the amine. Since the lone pairs of the nitrogen atoms are no longer able to interact with the acridine, the PET is hindered and the fluorophore is "switched on". The titration experiment with cadmium ions (Figure 1.11, right) can be analogously explained as well as for $\mathrm{Zn}^{2+}$ and $\mathrm{Hg}^{2+}$ ions, only less pronounced. As already mentioned, crystallographic studies were made with these complexes, discussing the free ligand ${ }^{[58]}$ and its metal complexes with $\mathrm{Ag}^{+}, \mathrm{Cu}^{2+}$, and $\mathrm{Cd}^{2+}$ ions, published in $2016^{[59]}$. The information of these publications are interesting, especially when 
comparing the fluorescence studies with the solid state data. The discussion of these results as well as the comparison with the structures prepared for this thesis is done in Chapter 2.2.14 and 2.3.2.

\subsubsection{Intramolecular charge transfer (ICT)}

Besides the comprehensively discussed PET process, another mechanism used for the detection of analytes is the intramolecular charge transfer (ICT). In this case, no aliphatic spacer is present. The ICT occurs from an electron donor to an electron acceptor, both included in one conjugated compound. $^{[15]}$ For this purpose, heteroaromatic systems are often used instead of pure hydrocarbon aromatic compounds. If excited by UV light, their dipole moments change significantly resulting in a large Stokes shift. ${ }^{[60]}$ Furthermore, interactions of the donor or acceptor with metal ions can change the photophysical properties of the fluorophores. The efficiency of the ICT process is altered and may result in a colorimetric respond of the compound. ${ }^{[61]}$

The fluorophore 2-(4'-N,N-dimethylaminophenyl)imidazo[4,5-b] pyridine (DMAPIP-b) demonstrates this mechanism and its sensor abilities were investigated by Krishnamoorthy et al. ${ }^{[62]}$ Several metal ions were added to a solution of DMAPIP-b in acetonitrile without influencing its emission to a great extent (Figure 1.12, left). Only in the case of $\mathrm{Zn}^{2+}$ ions, the bathochromic shift of the emission maximum is pronounced strongly that the colour of the fluorescent solution changes from blue to green.
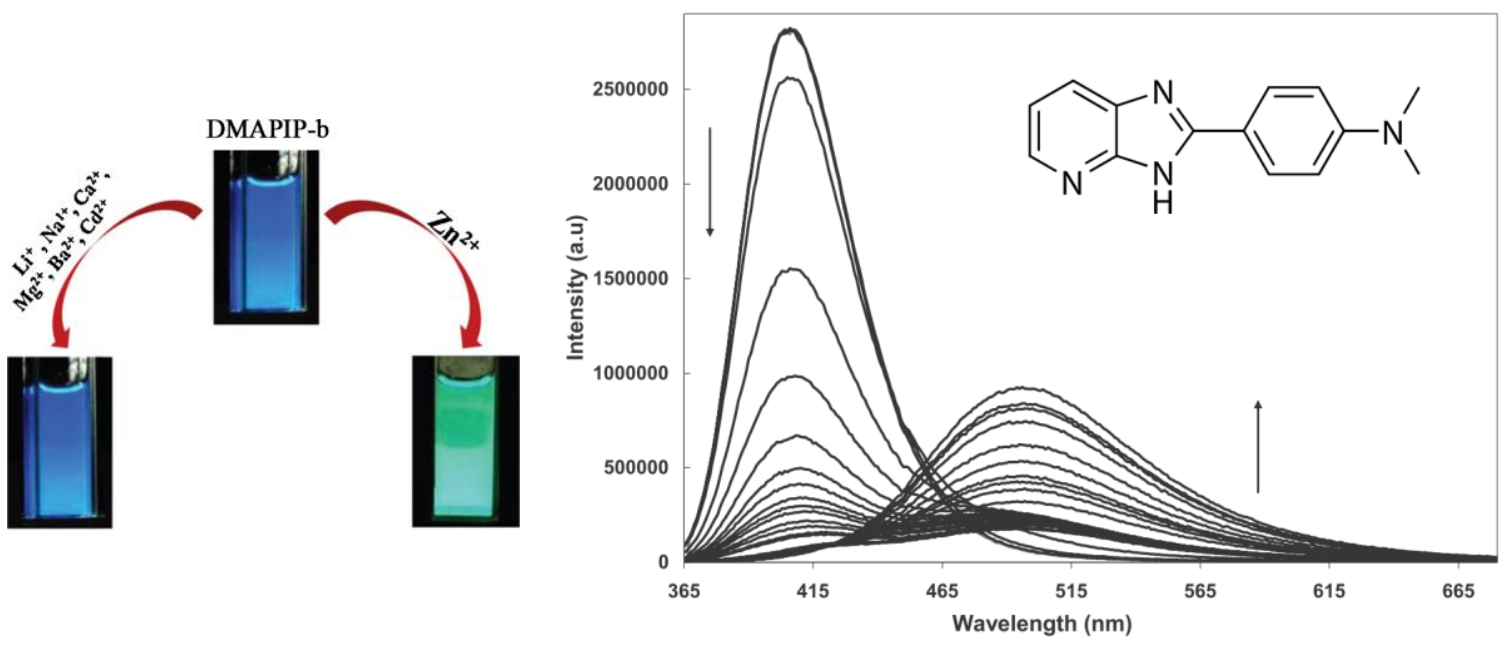

Figure 1.12: Left: Fluorescence of DMAPIP-b solutions in acetonitrile in presence of different metal ions. Right: Emission spectra of DMAPIP-b, illustrating the bathochromic shift upon addition of $\mathrm{Zn}^{2+}$ ions. ${ }^{[62]}$

The emission spectra in Figure 1.12, right, illustrate the red shift of the emission maximum of approximately $100 \mathrm{~nm}$ upon addition of $\mathrm{Zn}^{2+}$ ions. The authors state that the cation is coordinated by the pyridine nitrogen atom since this enhances the conjugation of the system. The coordination by the dimethylamine would have the opposite effect, resulting in a blue shift of the emission. This was confirmed by protonation experiments of the different nitrogen atoms. The colorimetric respond is a great advantage in the field of molecular sensors, enabling the detection of an analyte even by the naked eye.

Similar investigations were made in the Stalke group with anthracene derivatives. ${ }^{[25 \mathrm{e}]}$ The compound 9 -anthracene $(o$-( $\beta$-hydroxyethoxy)benzyl)amine was synthesised wherein the amine is 
directly linked to the anthracene allowing a strong electronic influence to the $\pi$-system. Fluorescence experiments have shown that the compound is able to chelate zinc ions in DCM. Thereby, the emission maximum is significantly blue-shifted $(100 \mathrm{~nm})$ along with an increase of intensity (Figure 1.13). This strong respond to the analyte can be attributed to the fact that "the fluorophore and the receptor share some critical atoms" ${ }^{[15]}$ which is a further requirement for ICT sensors.<smiles>OCCOc1ccccc1CNc1c2ccccc2cc2ccccc12</smiles>

A

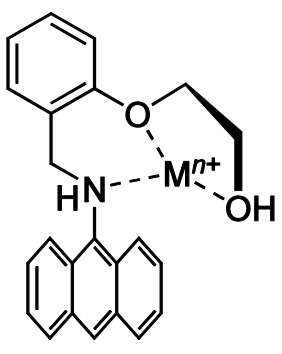

B

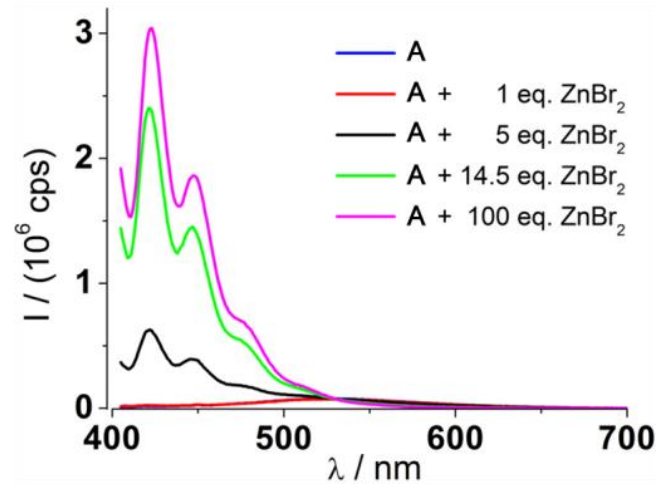

Figure 1.13: Left: Possible metal ion interaction of 9-anthracenepicolylamine. Right: Emission spectra of A with addition of $\mathrm{ZnBr}_{2}$ in $\mathrm{DCM}$. ${ }^{[25]}$

\subsection{Scope}

Former investigations of the Stalke group focussed on the hydrocarbon fluorophore anthracene. In this thesis, two representatives of the heteroaromatic congener azaanthracene with different fluorescence properties and potential usage as molecular sensors for metal cations were major fields of research. These compounds were 2- and 10-azaanthracene depicted in Figure 1.14, A and $\mathbf{B}, \mathbf{C}$, respectively.

2-Azaanthracene is a rare representative in the field of fluorescent sensors. Therefore, a suitable synthesis route for this aromatic system needed to be developed first. Its behaviour towards further substitution reactions to enable the "fluorophore-spacer-receptor" principle was of interest.<smiles>[R]c1c2ccccc2c([R])c2ccccc12</smiles>

A<smiles>[R]c1c2ccccc2nc2c([R16])cccc12</smiles>

B<smiles>[R12]Cc1cccc2c([R])c3cccc(N[R12])c3nc12</smiles>

C

Figure 1.14: Structures of different azaanthracene derivatives investigated in this thesis. The possible substitution patterns which were of major interest are illustrated.

Concerning the second representative 10-azaanthracene, two various fluorescence mechanisms should be studied. Depending on the existence of a spacer unit between the quencher and the fluorophore, either the photoinduced electron transfer (PET) or the intramolecular charge transfer (ICT) effect can be utilised for cation detection. The latter effect is feasible if the receptor is directly bonded to the aromatic system $(\mathbf{B})$ and has a strong electronic influence on the $\pi$-system. 
This is a rare motif in the acridine literature which is why different syntheses towards new 4-aminoacridine compounds were studied.

The PET effect is present if the structural design depicted in Figure 1.14, $\mathbf{C}$ is used. Well established experimental procedures have led to the publication of various analogous structures in the last few years. For the course of this thesis, a variety of amine receptors were employed to determine their influence to the selectivity and sensitivity of the molecular sensor devices. The synthesised compounds and their metal complexes needed to be structurally investigated in detail by NMR and fluorescence spectroscopy in solution as well as in solid state by X-ray diffraction. With these analytical methods, the chelating motif of the ligand towards the target ion can be investigated. Especially the possible coordination of the acridine nitrogen atom to the analyte, often discussed in the literature, should be examined. 



\section{RESULTS AND DISCUSSION}

\subsection{Investigations of 2-azaanthracenes}

During the prepended master thesis, the synthesis of pure 2-azaanthracene could be established. ${ }^{[63]}$ The reaction involves three steps with isolable intermediates. But with this unsubstituted aromatic unit, further conversions to the desired fluorophore-spacer-receptor systems would expend a high amount of substance. Since the employment of BMME to the congener acridine was of great success, the reagent was applied to 2-azaanthracene, too. Unfortunately, no desired product could be obtained by this procedure. In this case, the methylene groups serving as spacer units were integrated already during the synthesis of the azaanthracene.

\subsubsection{5,10-Dimethyl-2-azaanthracene (3)}

The already appropriated reaction by Krapcho and Gilmor ${ }^{[64]}$ during the master thesis will be used here in a modified way. Scheme 2.1 shows the first steps of the reaction pathway to 5,10-dimethyl-2-azaanthracene (3).<smiles>CC(=O)c1cccnc1</smiles><smiles>CC(=O)c1ccc[n+](C(=O)Oc2ccccc2)c1</smiles><smiles>CC(Br)c1ccccc1</smiles>

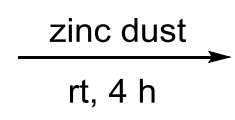<smiles>[C]C(Br)c1ccccc1</smiles>

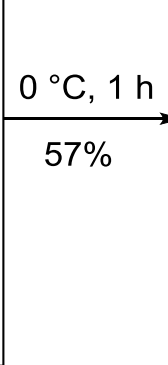<smiles>CC(=O)C1=CN(C(=O)Oc2ccccc2)C=C[C@H]1C(C)c1ccccc1</smiles>

Scheme 2.1: First part of the synthesis of 5,10-dimethyl-2-azaanthracene, showing the reaction to 1-phenoxycarbonyl-3-acetyl-4-phenylethyl-1,4-dihydropyridine (1). ${ }^{[64]}$

The modified starting materials provide the desired methyl groups highlighted in blue. In the upper part of the reaction, the protecting group phenyl formate is introduced to the nitrogen atom of the pyridine derivative. Additionally, this activates the species towards a Michael addition at position 4. In parallel, 1-bromo-1-phenylethane in THF was converted with zinc dust for four hours. After completion, the organozinc compound was added slowly to the pyridine derivative at $0{ }^{\circ} \mathrm{C}$ to obtain the diastereomeric intermediate 1 . After a column chromatographic work-up, the overall yield was 57\%. According to the three reaction steps, the average yield amounts to $83 \%$ each. The intermediate could be purified by column chromatography. A separation of the diastereomers was not necessary since in the following reaction step only one of the possible enantiomers is forming. With a look at the integral intensities of the proton signals in the NMR spectrum, the diastereomeric ratio is about 3:1.

The next two steps contained some difficulties during the procedure. Firstly, the cleavage of the phenoxycarbonyl group and aromatisation of the pyridine ring was performed by oxidative sulfur at high temperatures (Scheme 2.2, a). Due to these harsh conditions, several side products were formed which needed to be removed. Additionally, the high-boiling solvent had to be distilled off. 
<smiles>CC(=O)C1=CN(C(=O)Oc2ccccc2)C=C[C@H]1C(C)c1ccccc1</smiles>

1 a) $S_{8}$, decaline $190^{\circ} \mathrm{C}, 24 \mathrm{~h}$

b) conc. $\mathrm{HNO}_{3}$ $0{ }^{\circ} \mathrm{C} / \mathrm{rt}, 1 \mathrm{~h}$ $94 \%$<smiles>CC(=O)c1cnccc1C(C)c1ccccc1</smiles>

2 a) polyphosphoric acid $140{ }^{\circ} \mathrm{C}, 1 \mathrm{~h}$ $38 \%$

b) MSA rt, $20 \mathrm{~h}$ $92 \%$<smiles>Cc1c2ccccc2c(C)c2cnccc12</smiles>

3

Scheme 2.2: Further steps of the reaction pathway to $\mathbf{3}$ over the intermediate 2 , illustrating the reaction conditions by Krapcho and Gilmor ${ }^{[64]}$ (a) and the optimised conditions (b).

1 was dissolved in decaline together with sulfur and the mixture was heated to $190{ }^{\circ} \mathrm{C}$ for $24 \mathrm{~h}$. After a vacuum distillation, the dark sticky residue was extracted with dichloromethane and dried over $\mathrm{MgSO}_{4}$. The solvent was removed under reduced pressure to obtain a brown viscous oil. The yield of the synthesis of $\mathbf{2}$ was not determined due to some remaining decaline, but the ${ }^{1} \mathrm{H}$ NMR spectrum gave evidence that the conversion of the starting material was complete.

The last step was the acid catalysed cyclocondensation reaction of 2 with polyphosphoric acid. Both viscous reagents stirred sufficiently only at the needed high temperatures. After $1 \mathrm{~h}$ at $140{ }^{\circ} \mathrm{C}$, the reaction mixture was neutralised, extracted with DCM and purified by column chromatography. The product was obtained as a yellow powder but in poor yields. This is why the conditions of these last two steps were optimised. Instead of the oxidation by sulfur in a highboiling solvent, conc. $\mathrm{HNO}_{3}$ was used. It is a strong oxidative acid which already reacts at much milder conditions. Therefore, 1 was cooled down to $0{ }^{\circ} \mathrm{C}$ when conc. $\mathrm{HNO}_{3}$ was added slowly. It was stirred at room temperature for $45 \mathrm{~min}$ and poured onto ice afterwards. The crude product was extracted with DCM and also purified by column chromatography, to achieve a great yield of 94\%. Since it was not possible to determine the yield with the oxidation conditions by Krapcho and Gilmor, one cannot reference the value directly. But according to the literature yield of $44 \%$, the improvement is excellent. Additionally, an elaborate distillation of the solvent was not needed.

The use of polyphosphoric acid for the cyclocondensation reaction was not straightforward. The acid is highly viscous which impedes its handling and needs to be stirred sufficiently. The harsh reaction conditions can support the formation of undesired by-products which could be a reason for the low yield. An alternative reagent for this kind of cyclisation reactions can be found in the literature published by Leon and co-workers. ${ }^{[65]}$ They prefer using methanesulfonic acid (MSA) without heating. Therefore, 2 was dissolved in small amounts of MSA and was stirred at room temperature for 20 hours. After pouring onto ice, the solution was neutralised with $\mathrm{NaHCO}_{3}$ and extracted with DCM. Under these new conditions, a yield of $92 \%$ after column chromatographic work-up could be achieved. The first step of the synthesis had a moderate yield of 57\% (1) which reduced the overall yield of $\mathbf{3}$ to $49 \%$. This is still a sufficient value with respect to the initially challenges.

In Figure 2.1, the ${ }^{1} \mathrm{H}$ NMR spectrum of 5,10-dimethyl-2-azaanthracene (3) is depicted. It demonstrates the almost pure product with only slight impurities in the aromatic region as well as some residual solvent dichloromethane. With this precursor in hand, further substitution reactions at the methyl groups at the 5,10-positions were studied. 


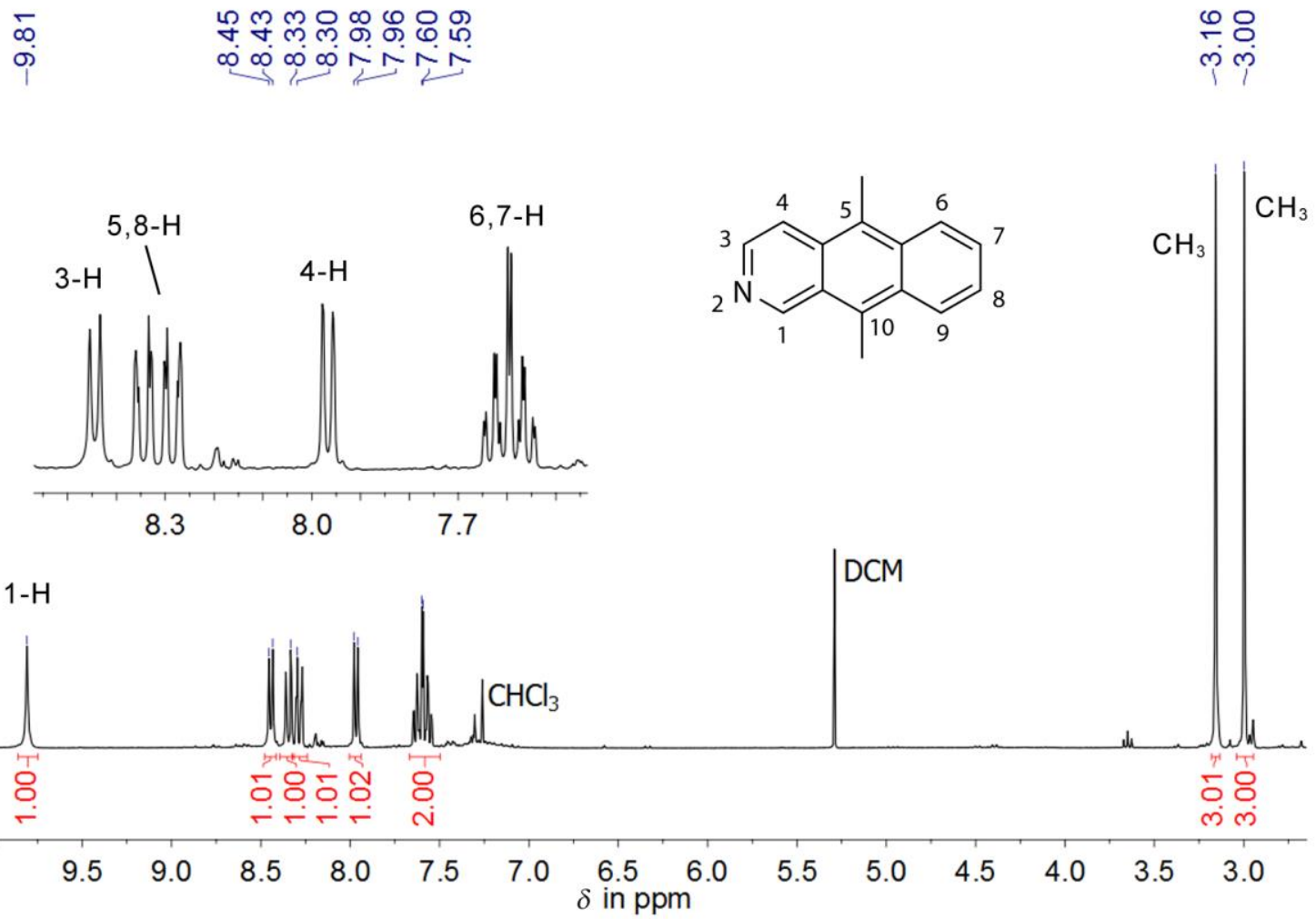

Figure 2.1: ${ }^{1} \mathrm{H}$ NMR spectrum of $\mathbf{3}$ in $\mathrm{CDCl}_{3}$.

\subsubsection{Investigations to the bromination of 5,10-dimethyl-2-azaanthracene (3)}

The next step for the build-up of a molecular sensor was the introduction of bromine atoms at the methyl groups, followed by a substitution reaction (Scheme 2.3). A common bromination of side arms like methyl groups can be realised with $N$-bromosuccinimide (NBS) and a radical initiator under high temperatures (Wohl-Ziegler reaction). ${ }^{[66]} \mathrm{NBS}$ is used because of its polarised $\mathrm{N}-\mathrm{Br}$ bond, leading to a partially positively charged bromine atom which can easily be cleaved. To initiate the radical reaction, benzoyl peroxide or tert-butyl peroxybenzoate has been used.<smiles>Cc1c2ccccc2c(C)c2ccccc12</smiles>

3<smiles>BrCc1c2ccccc2c(CBr)c2cnccc12</smiles>

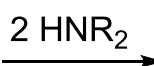<smiles>[R20]Cc1c2ccccc2c(CN[R20])c2cnccc12</smiles>

Scheme 2.3: Reaction pathway for the build-up of a molecular sensor system based on 3 .

Table 2.1: Different reaction conditions for the bromination approach of $\mathbf{3}$.

\begin{tabular}{ccccc}
\hline No. & Solvent & Temperature & Reaction time & Radical starter \\
\hline \hline 1 & $\mathrm{CHCl}_{3}$ & $70{ }^{\circ} \mathrm{C}$ & $5 \mathrm{~h}$ & $(\mathrm{PhC}(\mathrm{O}) \mathrm{O})_{2}$ \\
2 & $\mathrm{CHCl}_{3}$ & $70{ }^{\circ} \mathrm{C}$ & $22 \mathrm{~h}$ & $(\mathrm{PhC}(\mathrm{O}) \mathrm{O})_{2}$ \\
3 & $\mathrm{DMF}$ & $80^{\circ} \mathrm{C}$ & $4 \mathrm{~h}$ & $(\mathrm{PhC}(\mathrm{O}) \mathrm{O})_{2}$ \\
4 & $\mathrm{DMF}$ & $25{ }^{\circ} \mathrm{C}$ & $24 \mathrm{~h}$ & ${ }^{t} \mathrm{BuOOC}(\mathrm{O}) \mathrm{Ph}$ \\
\hline
\end{tabular}


The bromination was carried out under four different reaction conditions, listed in Table 2.1. Other than expected, none of the approaches resulted in sufficient amounts of the desired product. Mostly, the starting material decomposed, whereas in some cases slight traces of the bromomethylene compound could be identified via ${ }^{1} \mathrm{H}$ NMR spectroscopy. However, these small amounts could not be isolated and purified for further analytics. In the literature, the successful bromination of the similar compound 9,10-dimethylanthracene was demonstrated with NBS. ${ }^{\text {[66a] }}$ According to this, the nitrogen atom of the azaanthracene seems to hinder the radical conversion by unintended side reactions. However, the reaction of 9-methyl-10-azaanthracene with NBS, which is discussed in Chapter 2.2.2 of this thesis, resulted in the desired bromination of the methyl group.

A preceding oxidation of the aromatic nitrogen atom may prevent the decomposition of $\mathbf{3}$. After the conversion with NBS, the nitrogen atom could be reduced with the oxophilic $\mathrm{PPh}_{3}$. Unfortunately, this hypothesis could not be investigated due to the lag of starting material based on its difficult synthesis. However, this would extend the synthesis of potential molecular sensors by two more steps, resulting in an overall reaction pathway including seven steps. This elaborate synthesis as well as the susceptible intermediates led to the decision to no longer pursue this type of azaanthracene. During the preceding master thesis, successful experiences were gained with the congener 10-azaanthracene which is why the focus was set on this fluorophore.

\subsection{Potential molecular sensors based on acridine}

The reactivity of acridine towards different reagents such as nucleophiles or electrophiles has been reviewed earlier by Acheson ${ }^{[67]}$ and lately by Galy and Chiron ${ }^{[68]}$. The relevant positions for this thesis are 4, respectively 5, and to a certain extent the 9-position. In Figure 2.2, the frontier electron density for electrophilic substitution is shown.
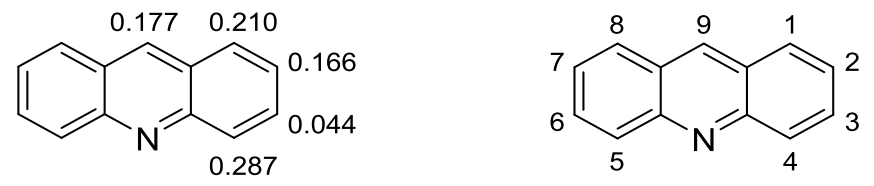

Figure 2.2: Calculated $\pi$-electron densities of acridine ${ }^{[69]}$ and its IUPAC numbering used in this thesis.

Electrophilic attacks should primarily occur at position 4 which is enhanced by the protonation of the nitrogen atom by an acid. It was taken advantage of this behaviour in the course of work for this thesis. In contrary, position 9 of acridine can be attacked by nucleophiles due to its lower electron density. This is attributed to the nitrogen atom at the para-position of the central ring.

\subsubsection{9-Methylacridine (4)}

On the one hand, the introduction of a methyl group at the 9-position can be achieved by a nucleophilic substitution with e.g. organolithium compounds at the aromatic system. ${ }^{[70]}$ On the other hand, a cyclocondensation reaction can be carried out to build-up the desired acridine derivative. The use of diphenylamine with either formic or acetic acid results in the synthesis of acridine and 9-methylacridine, respectively (Scheme 2.4). ${ }^{[36 \mathrm{~b}]}$ 


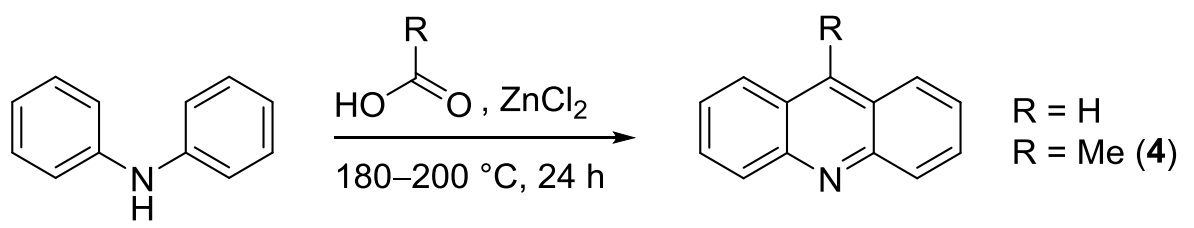

Scheme 2.4: Bernthsen synthesis of the fluorophores acridine and 9-methylacridine (4). ${ }^{[36 \mathrm{~b}]}$

Whereas pure acridine is commercially available and was not synthesised in this thesis, its 9-methyl derivative 4 was obtained by the use of 3 eq of glacial acetic acid and 4 eq of zinc chloride. High temperatures around $200{ }^{\circ} \mathrm{C}$ are needed for the cyclocondensation of the reagents. That is why the amount of acetic acid must be minimised due to its boiling point of $118{ }^{\circ} \mathrm{C}$. Diphenylamine melts at $53{ }^{\circ} \mathrm{C}$ and acts as reagent as well as solvent. Mechanic stirring was needed to achieve a sufficient mixture of the starting materials. $\mathrm{ZnCl}_{2}$ is used as Lewis acid and stabilises the intermediate. At the beginning of the reaction, the molten diphenylamine dyes the reaction mixture violet, whereas it changes the colour to dark green at temperatures above $180{ }^{\circ} \mathrm{C}$. After one day, it was cooled to room temperature and the reaction was stopped by the addition of aqueous $\mathrm{NaOH}$ solution $(2 \mathrm{M})$. The organic compounds were extracted with DCM and dried over $\mathrm{MgSO}_{4}$. The obtained product was of sufficient pureness with a yield of $57 \%$.

The introduction of the methyl group had several reasons. On the one hand, it enhances the electron density of the aromatic system due to its positive inductive effect. This could improve the yields for further electrophilic reactions at the positions 4 and 5 which are discussed in Chapter 2.2.4. With regard to the nitrogen atom, a higher electron density could improve its donating properties for the coordination of metal cations, but only to a small extend. On the other hand, the methyl group can be utilised for further substitution reactions. For example, chelating amines can be used to coordinate target ions on both sides of the fluorophore - at position 9 and opposite to it at the 4- and 5-positions.

In the following, several attempts to further derivatives of acridine at the 9-position are discussed. They all originate from the previously shown reaction with diphenylamine as the starting material and an excess of $\mathrm{ZnCl}_{2}$.

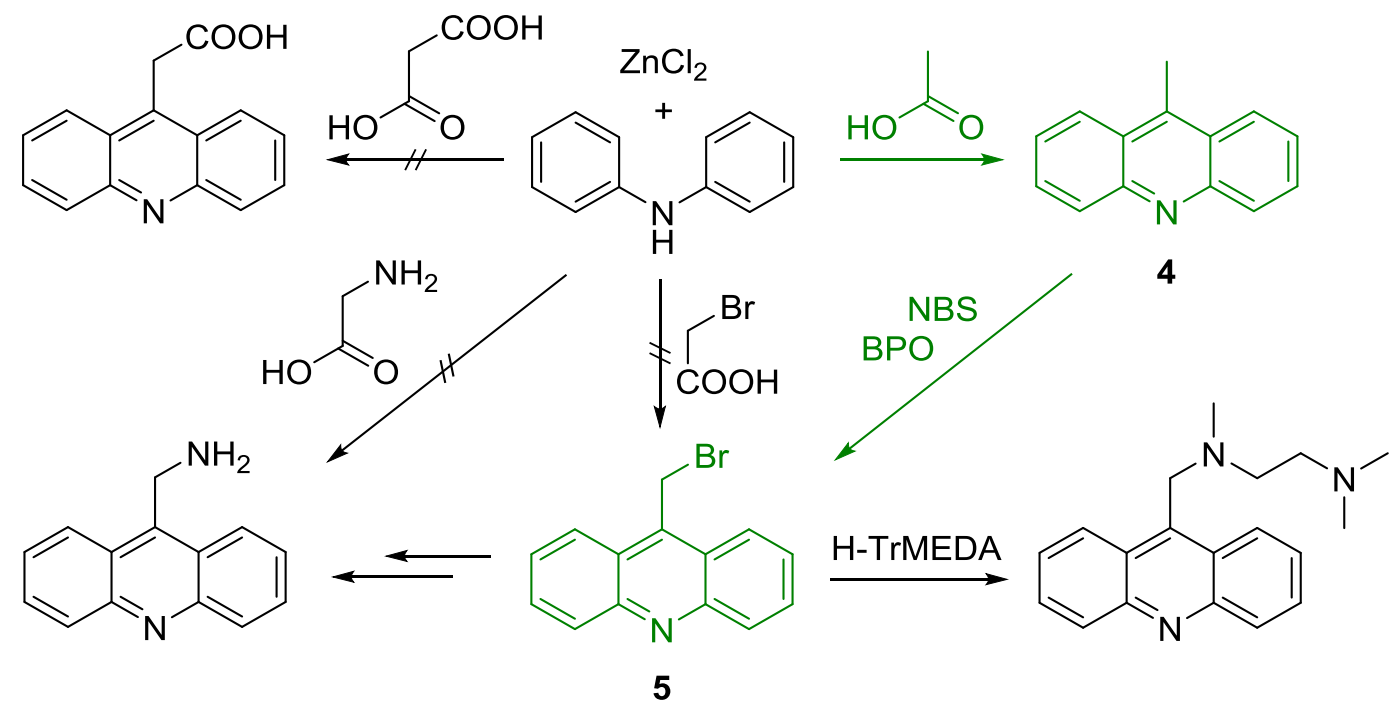

Scheme 2.5: Overview to a variety of potential compounds originated from diphenylamine. 
Various reagents have been used to introduce functional groups in the para-position of the nitrogen atom by cyclocondensation reactions (Scheme 2.5). Unfortunately, most of them did not yield the desired product. In the case of glycine, no conversion of the starting material was observed, whereas the use of malonic acid only yielded 9-methylacridine likewise to the Bernthsen reaction with acetic acid. The latter can be explained by a decarboxylation of one of the carboxylic acid groups during the reaction. The achieved yield was lower in comparison to the standard procedure of Scheme 2.4.

\subsubsection{9-(Bromomethylene)acridine (5)}

A direct approach for the synthesis of $\mathbf{5}$ was tried with three equivalents of bromoacetic acid, depicted in Scheme 2.5. By this, an intermediate should be obtained which is convertible to potential molecular sensors by a following substitution reaction with e.g. amines. In the beginning of the reaction, the reagents were dissolved in the melted diphenylamine. After one hour at $200{ }^{\circ} \mathrm{C}$, the reaction mixture became solid and it was cooled to room temperature. Most of the compound was dissolved in methanol and residues were filtrated off. The ${ }^{1} \mathrm{H}$ NMR spectrum showed that the introduction of the bromomethylene unit was not feasible by this method.

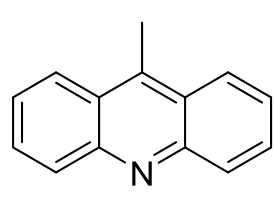

4

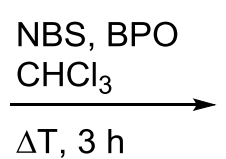

$\Delta \mathrm{T}, 3 \mathrm{~h}$

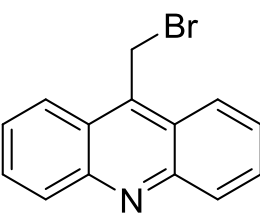

Scheme 2.6: Synthesis of 9-(bromomethylene)acridine (5).

With the use of $\mathbf{4}$ as precursor and $N$-bromosuccinimide as reagent, a second route to 9-(bromomethylene)acridine was performed. Both starting materials were dissolved in chloroform in equimolar amounts when $7 \mathrm{~mol} \%$ of the radical initiator benzoyl peroxide (BPO) was added. The solution was heated to the boiling point of the solvent for three hours. After the solvent was removed under reduced pressure, a recrystallisation from ethanol was implemented to remove residual succinimide. After a column chromatographic work-up, 5 was obtained in a yield of $55 \%$.

The bromine should now be replaced by an amine group. A primary amine can be obtained by the use of urotropine and hydrochloric acid. ${ }^{[71]}$ Afterwards it can be derivatised with e.g. aldehydes. Here, the direct substitution by a secondary amine was tried (Scheme 2.5). The amine $N, N^{\prime}, N^{\prime}$ trimethylethylenediamine (H-TrMEDA) was chosen for the substitution of the bromine since it contains two nitrogen atoms for the coordination of an analyte. The three methyl groups enhance the donor ability of the diamine. For the conversion of $\mathbf{5}$, one equivalent of the amine and an excess of $\mathrm{K}_{2} \mathrm{CO}_{3}$ was added. The basic salt improves the reaction by deprotonating the amine and it precipitates the bromide as $\mathrm{KBr}$. The reaction was performed in chloroform overnight at a temperature of $50{ }^{\circ} \mathrm{C}$. A ${ }^{1} \mathrm{H}$ NMR spectrum was recorded after 18 hours. It showed a significant upfield shift of the intense singlet of the $\mathrm{CH}_{2}$ group by almost one ppm. This was a good indicator for the exchange of the bromine with a nitrogen atom. Furthermore, new signals in the aromatic region were found, whereas the spectrum indicated at least two present derivatives. To separate these, a column chromatography on alumina was applied. During the work-up, further derivatives were observed by the use of thin layer chromatography. Several fractions were isolated and 
investigated by NMR spectroscopy. Unfortunately, no signals which belonged to the desired product were identified. Either the reaction did not yield the TrMEDA-substituted acridine or the chromatographic procedure led to the decomposition of the compound.

The main focus of this thesis laid on the determination of 4-mono- and 4,5-disubstituted acridine amines. This is why the derivatisation of the 9-position was not investigated further.

\subsubsection{Attempts to 4-aminoacridine}

In the introduction, the molecular requirements for an intramolecular charge transfer (ICT) and its mechanism was discussed. In comparison to the methylene bridged derivatives mentioned in the chapter before, the receptor unit needs to be directly attached to the fluorophore. This was investigated in the Stalke group with anthracene derivatives. ${ }^{[25 \mathrm{e}]}$ The molecular sensor 9 -anthracene( $o$-( $\beta$-hydroxyethoxy)benzyl)amine was synthesised and its sensor abilities were determined. The latter was already discussed in the introduction and its synthesis is presented here in detail.

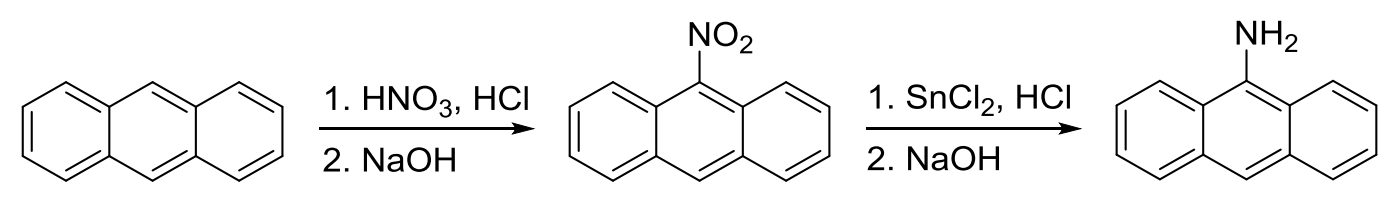<smiles>[R]CNc1c2ccccc2cc2ccccc12</smiles>

Scheme 2.7: Reaction pathway to molecular ICT sensors based on 9-aminoanthracene. ${ }^{[25]}$

Wandtke used nitric acid for the nitration of anthracene at the 9-position, with a following reduction to the amine with $\mathrm{SnCl}_{2}$ and $\mathrm{HCl}$ (Scheme 2.7). Based on this intermediate, different types of molecular sensors were synthesised. The reaction pathway should be adopted to acridine as fluorophore. Here, the amine should be introduced in the 4-position of acridine which allows to coordinate an analyte by the amine as well as by the aromatic nitrogen atom. This possibility is an advantage in comparison to the hydrocarbon congener anthracene.

The nitration of acridine has been studied by different researchers with modest results. The electrophilic nitration of the aromatic system not bearing directing substituents often leads to mixtures of nitrated products along with the oxidation of the starting material. ${ }^{[72]}$ In most cases, multiple nitration occurs at the aromatic system. ${ }^{[73]}$ Since the numbering of acridine is frequently mixed up in earlier literature ${ }^{[74]}$ and the identification of products was mainly realised by elemental analysis ${ }^{[75]}$, it often not became clear which nitro-substituted derivative was synthesised. However, by summarising different approaches, Lehmstedt figured out that changes of the reaction temperature and the ratio of the used acids yielded different substituted acridines. ${ }^{[76]}$ Therefore, various attempts to nitroacridine derivatives were performed which are listed in Scheme 2.8. 


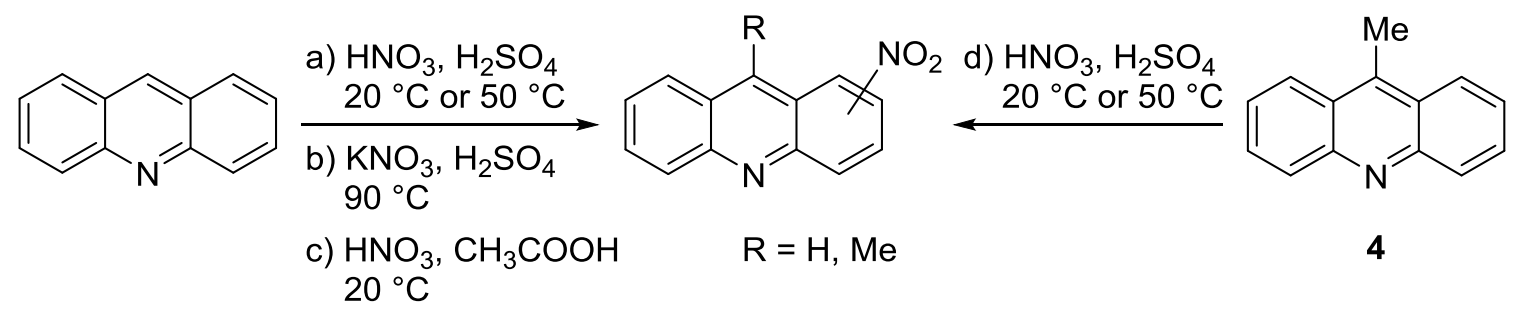

Scheme 2.8: Different attempts of the nitration of (9-methyl)acridine.

The commonly used reagents for the nitration of aromatic systems are nitric acid in combination with sulfuric acid. The resulting nitronium ion is able to perform electrophilic attacks. According to the calculated $\pi$-electron densities of acridine shown in Figure 2.2, the nitration should take place in position 1 and 4. In general, the aromatic compound was dissolved in acid and heated to the appropriate temperature. The reaction was stopped by pouring onto ice and the precipitates were filtrated off and washed with water. $\mathrm{NH}_{3}$ or aqueous $\mathrm{NaOH}$ was added to the filtrate until it became alkaline. Further possible precipitates were filtrated and the solid products were dissolved in organic solvents. The solution was dried over $\mathrm{MgSO}_{4}$ and the volatile components were removed under reduced pressure after filtration. In the case of procedure a) and d), the ${ }^{1} \mathrm{H}$ NMR spectrum was highly crowded. The crude product from procedure a) was recrystallised from glacial acetic acid resulting in a small amount of residue which could be characterised by NMR spectroscopy (Figure 2.3).

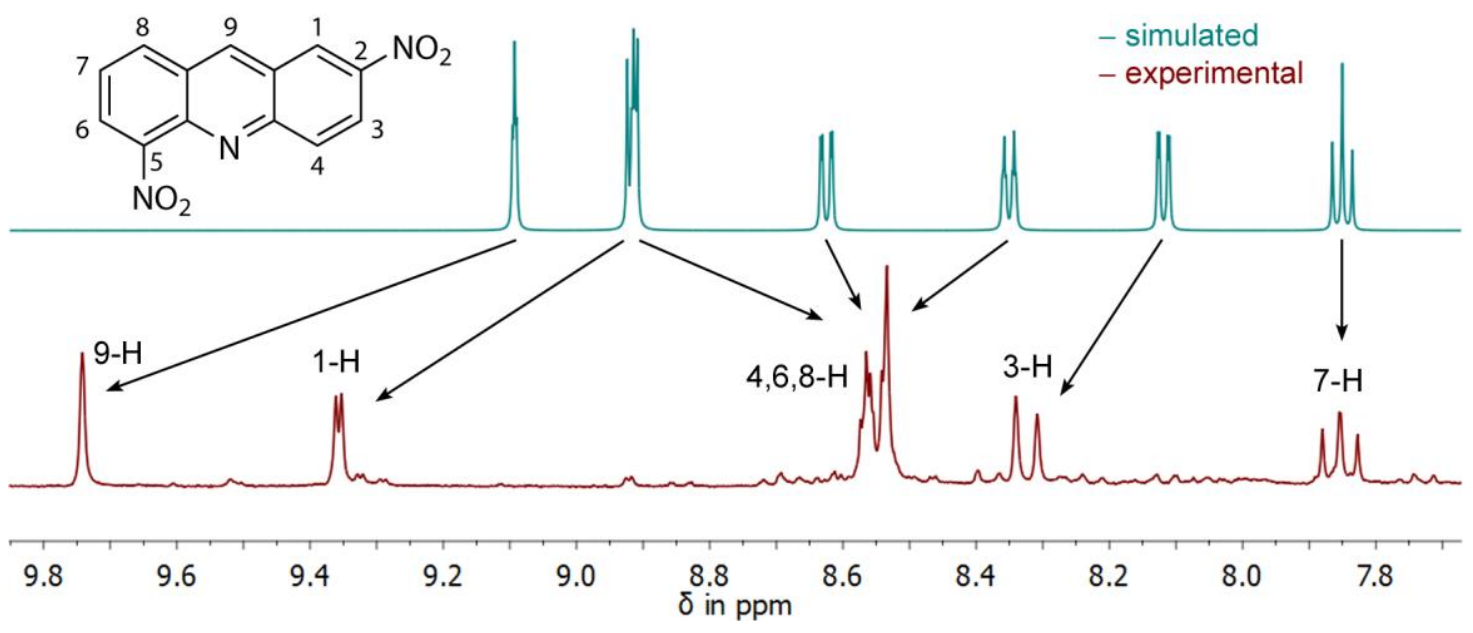

Figure 2.3: ${ }^{1} \mathrm{H}$ NMR spectrum of the proposed 2,5-dinitroacridine (red) and its simulated spectrum (turquoise).

The signals of the spectrum could be assigned to the relative protons with the help of a H,H-COSY NMR experiment and due to their multiplicity and chemical shift. Therefore, the measured spectrum was compared with a simulated spectrum calculated by the MestreNova ${ }^{\circledR}$ software (v6.0.2). The integral intensities of the signals confirm the presence of seven protons. These results indicate that the disubstituted 2,5-dinitroacridine was isolated from the mixture of nitrated products.

With regard to the poor yields, the reaction conditions were modified. Nevertheless, the use of $\mathrm{HNO}_{3}$ in glacial acetic acid did not lead to any reaction with the aromatic system. A further variation was the change of $\mathrm{HNO}_{3}$ to its salt $\mathrm{KNO}_{3}$, also combined with $\mathrm{H}_{2} \mathrm{SO}_{4} \cdot{ }^{[77]}$ Likewise to the prior attempts, the ${ }^{1} \mathrm{H}$ NMR spectrum shows a variety of nitrated derivatives. After the usual 
work-up, a column chromatography was performed to separate the different species. Due to their similar physical properties, no sufficient results were obtained. Only one fraction contained a disubstituted product which was analysed by NMR spectroscopy and compared with the simulated spectrum (Figure 2.4).

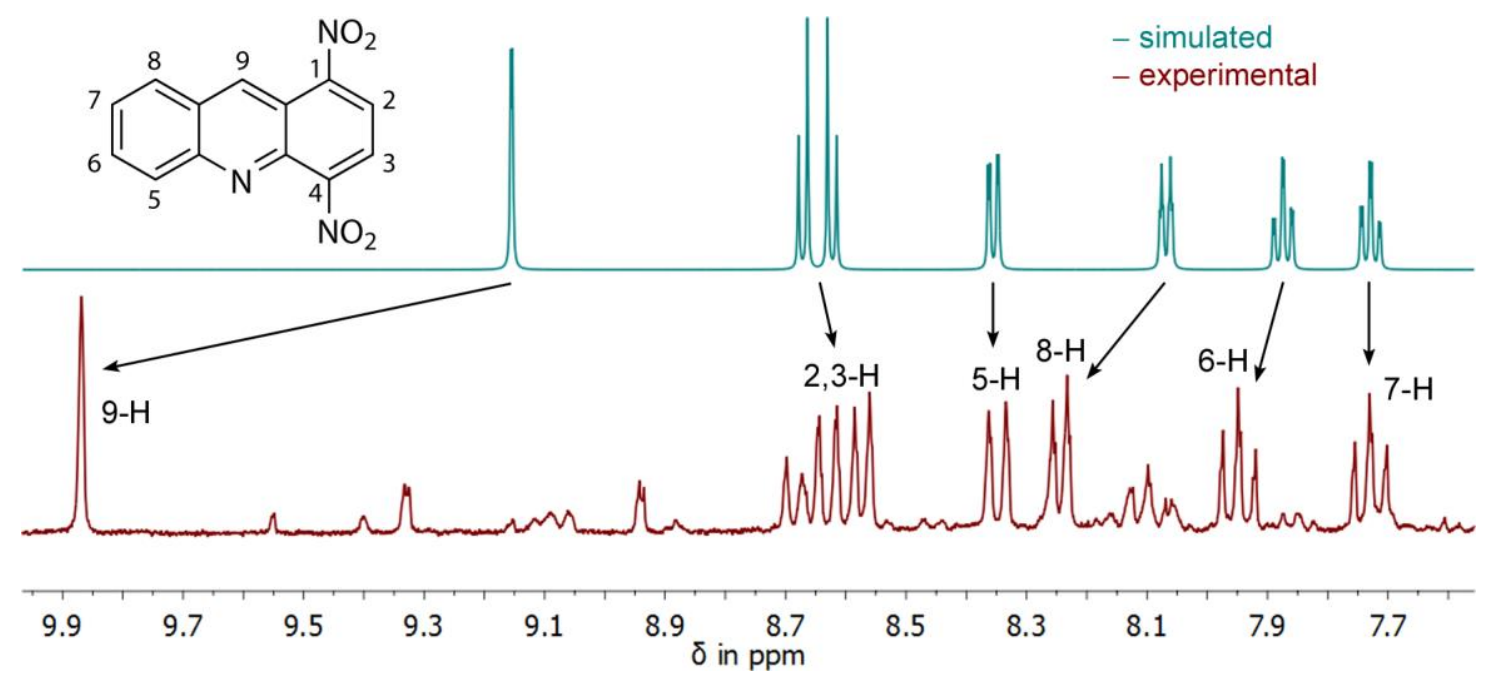

Figure 2.4: ${ }^{1} \mathrm{H}$ NMR spectrum of the proposed 1,4-dinitroacridine (red) and its simulated spectrum (turquoise).

In this case, the 1,4-dinitroacridine was formed which was determined in the same manner like the derivative described before. The analysis of the chemical shift, the integral intensity, and mainly the multiplicity of the signals resulted in the assumed compound. Again, only a small amount of the compound was isolated which still needs to be purified. This is why further attempts to the direct nitration of acridine were not followed although the substitution of the desired position 4 , respectively 5 , was successful.

Another route for the introduction of an amino group is the use of appropriate starting materials for condensation reactions, yielding the desired acridine derivatives. One approach was the use of 2-aminodiphenylamine which should be able to undergo a condensation reaction with formic acid to 4-aminoacridine. However, the primary amino group would react with formic acid to form 1phenyl-1H-benzimidazole. ${ }^{[78]}$<smiles>Nc1ccccc1Nc1ccccc1</smiles><smiles>CCO[Sb]([O-])(Cl)(Cl)c1ccc([N+](=O)[O-])cc1</smiles><smiles>Nc1ccccc1[N+](=O)[O-]</smiles>
Cul, $\mathrm{K}_{2} \mathrm{CO}_{3}$ $L$-proline $125^{\circ} \mathrm{C}, 48 \mathrm{~h}$

$$
\longrightarrow
$$<smiles>[R2]Nc1ccccc1Nc1ccccc1</smiles><smiles>[R10]c1cccc2cc3ccccc3nc12</smiles>

6: $R=$ Nos

7: $\mathrm{R}=\mathrm{O}$

Scheme 2.9: Two different approaches to derivatives of 2-aminodiphenylamine. 
On the one hand, this unwanted reaction can be hindered by the use of a suitable protection group. On the other hand, a nitro group can be used instead which is afterwards easily reducible to the amine. Both methods were tried which synthetic routes are depicted in Scheme 2.9. 4-Nitrobenzenesulfonyl chloride was chosen as a suitable protecting group for the amine since it is stable towards acids which are used for the preceding cyclocondensation reaction. Triethylamine was added as base and the reaction mixture was stirred in DCM for one hour at room temperature. The protected amine 6 was isolated with a yield of $74 \%$. It was tried to react this intermediate with formic acid in the presence of $\mathrm{ZnCl}_{2}$ to form the 4-substituted acridine derivative. In this case, the two large protecting groups increase the melting point of the diphenylamine derivative drastically. Therefore, the solid reagents did not react sufficiently even at temperatures of $190{ }^{\circ} \mathrm{C}$ for $12 \mathrm{~h}$.

2-Nitrodiphenylamine (7) has a melting point of $75^{\circ} \mathrm{C}^{[79]}$ Its synthesis was adopted from the proceedings of $\mathrm{Fu}$ et al. who studied the reactions of aniline and bromobenzene derivatives. ${ }^{[80]}$ The authors made use of the catalysts $\mathrm{CuI}(10 \mathrm{~mol} \%)$ and $L$-proline $(20 \mathrm{~mol} \%)$ which enables the conversion of the starting materials in the presence of a base and temperatures around $110{ }^{\circ} \mathrm{C}$. High boiling and polar solvents like DMF or DMSO were used in the publication. In the course of work for this thesis it has been founded that these conditions need very long reaction times. This can be reduced by higher temperatures, whereas $150{ }^{\circ} \mathrm{C}$ led to an unknown side reaction. A temperature of $125^{\circ} \mathrm{C}$ was chosen along with the use of bromobenzene as solvent, which forces the equilibrium of the reaction to the side of the products. After $48 \mathrm{~h}$, the crude product was purified by column chromatography to give 7 in a yield of $67 \%$. Likewise to $\mathbf{6}$, its reaction to 4-nitroacridine by the use of the Bernthsen procedure was tested. During the reaction with 3 eq of formic acid, a dark brown solution was formed when the reaction mixture was heated to $190{ }^{\circ} \mathrm{C}$. After $4 \mathrm{~h}$, a black solid remained which is why additional $3 \mathrm{eq}$ of formic acid were added and stirred for two more hours. A ${ }^{1} \mathrm{H}$ NMR spectrum was recorded from a sample of this crude, black compound. Unfortunately, the spectrum showed the complete decomposition of the starting material. The nitro group is most probably not stable towards high temperatures which are needed for the cyclocondensation of the reagents.
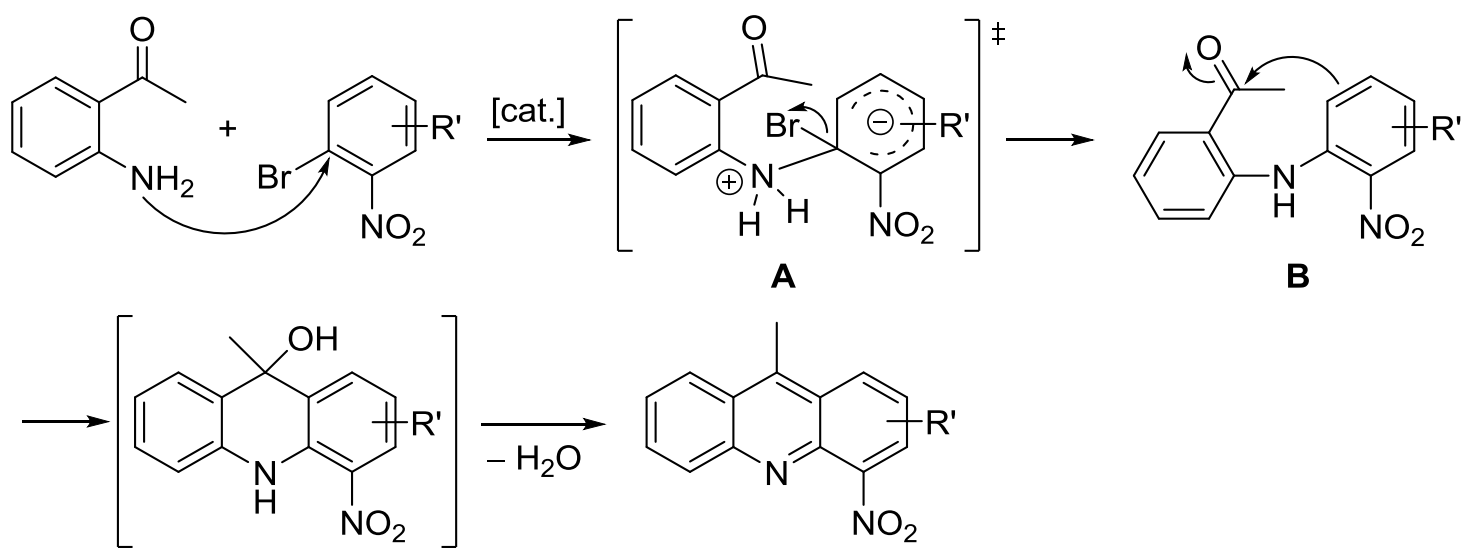

Scheme 2.10: A possible approach to 9-methyl-4-nitroacridine derivatives via intramolecular condensation reaction.

To avoid these harsh reaction conditions, it was tried to enhance the reactivity of the diphenylamine derivative. If an aldehyde or ketone group was attached to the ortho-position, an intramolecular, nucleophilic attack from the neighboured phenyl ring would be possible. This is 
depicted in Scheme 2.10, where 2-acetylaniline and 1-bromo-2-nitrobenzene are used as starting materials. The electron-withdrawing nitro group in ortho-position to the bromine atom enhances the nucleophilic attack of the amine. Furthermore, it stabilises the negative charge of the transition state A. After the cleavage of the bromide, the second nucleophilic attack to the carbon atom of the ketone should have a smaller activation energy than former intermolecular reactions with formic acid. Various attempts to the formation of intermediate $\mathbf{B}$ were investigated and are listed in Table 2.2.

Table 2.2: Different attempts to react ortho-substituted phenylamines with bromobenzene derivatives.

\begin{tabular}{l} 
reagents \& solvent \\
\multicolumn{2}{c}{$\begin{array}{c}\text { reaction conditions } \\
\text { temp. }\end{array}$} \\
time
\end{tabular}

For the first two entries, the catalysts and solvents were chosen according to the mentioned investigations by $\mathrm{Fu}$ et al. ${ }^{[80]}$ They used similar starting materials but none of them contained an acetyl group at the aromatic systems. The temperature and reaction times have been varied as well as the substitution pattern of the starting materials. The reaction process of the first and second entry was discussed in Scheme 2.10. In all cases, ${ }^{1} \mathrm{H}$ NMR spectra were recorded of the reaction mixtures. Entry 1 only yielded an unwanted side product whereas 2 showed no conversion of the starting materials, independent of the applied reaction time and temperature. In the third entry, ortho-phenylenediamine and bromobenzoic acid were used as starting materials. Additionally, the catalyst was changed to $\mathrm{Cu}^{0}$ according to literature procedures. ${ }^{[81]}$ Here, all ${ }^{1} \mathrm{H}$ NMR signals of the starting materials were dissipated after $3 \mathrm{~h}$, but the spectrum of the reaction mixture was too crowded to identify one define product. Since the desired diphenylamine derivative is a dipolar ion, the separation from salts and other byproducts was not feasible. In a second approach, the crude product was directly treated with concentrated $\mathrm{H}_{2} \mathrm{SO}_{4}$ after the removal of the solvent and heated to $110^{\circ} \mathrm{C}$ for $2 \mathrm{~h}$. The strong and hygroscopic acid should favour the cyclocondensation reaction, ${ }^{[81 a]}$ however, only the decomposition of the assumed intermediate was observed.

A variety of different attempts to obtain a 4-substituted acridinyl amine were performed. Neither the derivatisation of the acridine system nor the step-by-step formation of suitable intermediates led to the desired compounds. In some cases, the synthesis of intermediates was successful but the purification was too elaborate or the subsequent reaction was not realisable. Kitahara et al. 
already published the reaction of intermediate $\mathbf{B}$ to the product $\mathbf{C},{ }^{[82]}$ however, they did not describe the synthesis of the starting material. Additional investigations of these types of reactions need to be done in the future but will not be discussed further in this thesis.

In the following chapters, the synthesis of acridine sensor systems is approached in a different way. A methylene unit between the fluorophore and the receptor allows the use of a more feasible and explored reaction pathway.

\subsubsection{4,5-Bis(bromomethylene)acridine (8) and 4,5-bis(bromomethylene)-9-methyl- acridine (9)}

For the synthesis of the fluorophore-spacer-receptor systems, the first challenge was the introduction of the methylene group, acting as the spacer unit. During the last years, the Stalke group has performed intense research in the field of anthracene based fluorophores. ${ }^{[25]}$ Thereby, a lot of different derivatives containing a methylene spacer unit were developed and synthesised. The reaction pathway to the desired anthracene systems was often realised in the following route (Scheme 2.11).

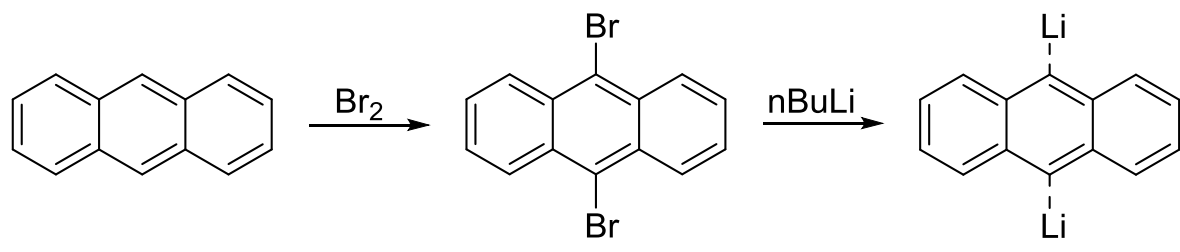<smiles>Cc1c2ccccc2c(C)c2ccccc12</smiles>

Scheme 2.11: Synthesis route to an anthracene precursor for molecular sensors.

This synthetic concept to the precursor 9,10-bis(bromomethylene)anthracene contains four steps, which implies a high synthetic effort. The bromide acts as a good leaving group in subsequent substitution reactions. Furthermore, they activate the methylene group towards nucleophilic attacks, due to their electron withdrawing properties. That is why in this thesis, the bromomethylene groups should be introduced to the acridine structure as well, but in a more efficient way.

This intention was reported by Galy and Chiron in 2003, already mentioned in the introduction. ${ }^{[41]}$ They made use of the reagent bromomethyl methyl ether (BMME), which is able to introduce one or two bromomethylene units to the aromatic system - in one step. The reaction needs to be carried out under strong acidic conditions. By that, the nitrogen atom of the acridine gets protonated and activates the neighboured positions 4 and 5 towards electrophilic substitution. Additionally, the halomethylether gets protonated at the oxygen atom as well, which supports the cleavage of the molecule (Scheme 2.12). With less than two equivalents of BMME and a temperature of $20^{\circ} \mathrm{C}$, the monosubstituted product can be obtained but only in small yields. When an excess of the ether is used (4 eq) with temperatures around $50{ }^{\circ} \mathrm{C}$, the disubstituted acridine is 
formed in a yield of $64 \% .{ }^{[41]}$ The latter is the more interesting derivative which synthesis has already been studied in former investigations, ${ }^{[63]}$ but will again be described below. Furthermore, with the use of 9-methylacridine as starting material, the methyl derivative of the described product has been obtained, too.

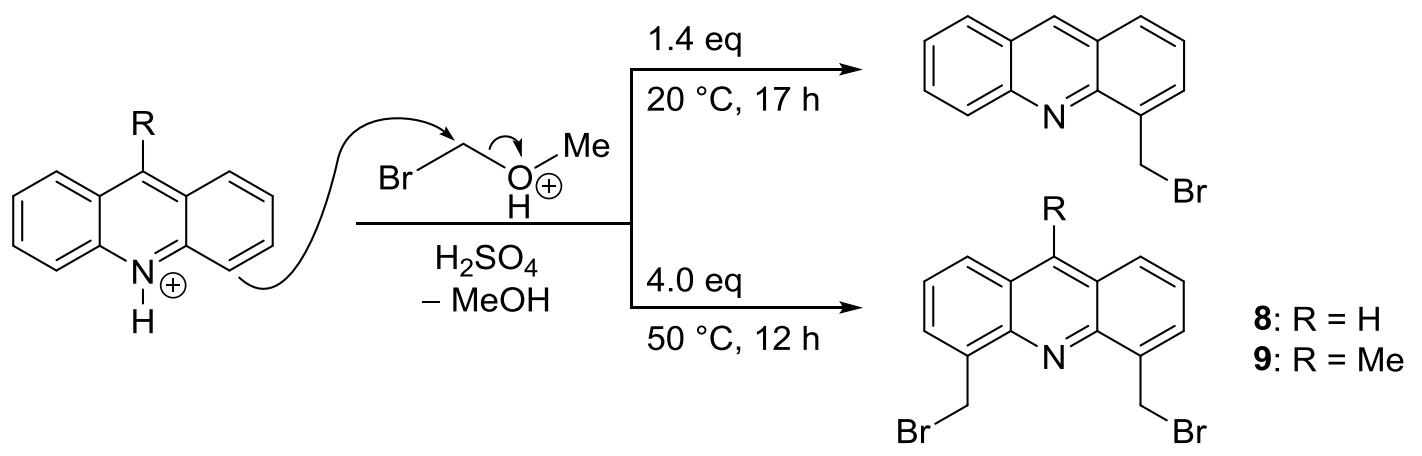

Scheme 2.12: Usage of bromomethyl methyl ether for the synthesis of 4,5-bis(bromomethylene)acridine (8) and its 9-methyl derivative 9.

For both preparations, acridine was dissolved in concentrated sulfuric acid and heated up to $50{ }^{\circ} \mathrm{C}$ when four equivalents of BMME were added. During the reaction time of $17 \mathrm{~h}$, a significant amount of bromine gas was formed. The use of the strong acid could cause the formation of hydrogen bromide which again can be oxidised to bromine. This possible side reaction makes an excess of the added halomethyl ether useful. The crude yellowish brown product was crushed out by pouring onto ice, after what it was filtered and washed with dem. water. It was dissolved in chloroform, washed with brine and dried over $\mathrm{MgSO}_{4}$. According to the literature, 8 can be purified by recrystallisation from $\mathrm{Et}_{2} \mathrm{O}$. In some cases, this procedure was almost sufficient, however, a repeated recrystallisation from chloroform was more satisfying. With the latter method, the yield drops down to the half of the reported value of $64 \%$, but resulted in a purer product which was of higher importance with regard to the subsequent reactions. For the work-up of 9, a column chromatography was applied (PE/EA, 10:1) which gave the desired product in a yield of 54\%. The higher yield of $\mathbf{9}$ in comparison to $\mathbf{8}$ may be a result of the methyl group which enhances electrophilic reactions at the aromatic system.

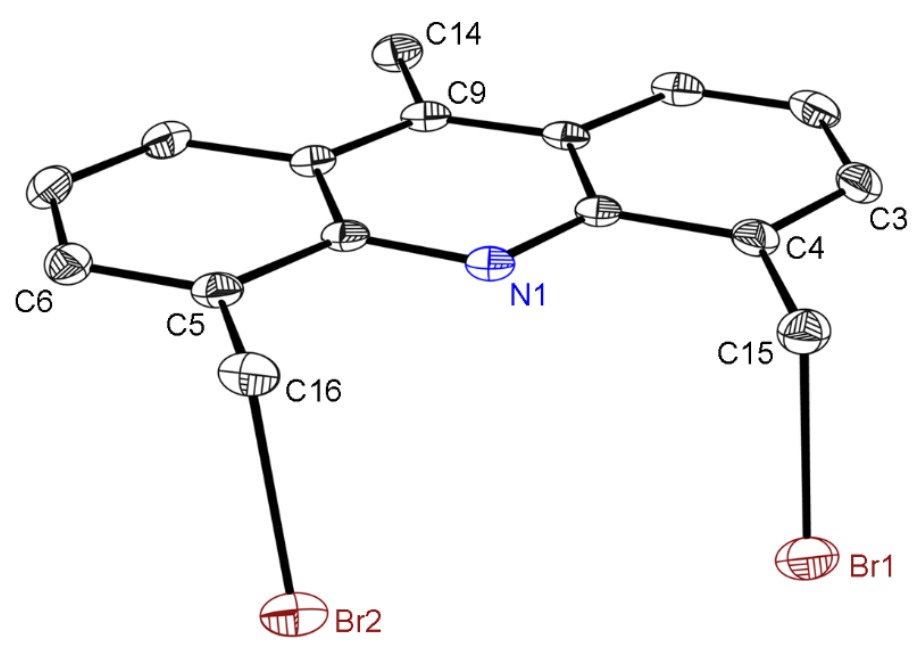

Table 2.3: Selected bond lengths [pm] and angles $\left[{ }^{\circ}\right]$ of 9.

\begin{tabular}{cc}
\hline Br1-C15 & $198.03(18)$ \\
Br2-C16 & $197.52(17)$ \\
C9-C14 & $151.0(2)$ \\
C4-C15-Br1 & $110.28(12)$ \\
C5-C16-Br2 & $111.72(12)$ \\
C3-C4-C15-Br1 & $-92.33(18)$ \\
C6-C5-C16-Br2 & $97.52(17)$ \\
\hline
\end{tabular}

Figure 2.5: Solid state structure of 9 illustrating the asymmetric unit. 
In the case of 9 , a recrystallisation from DCM and a storage at $-30{ }^{\circ} \mathrm{C}$ gave suitable crystals for $\mathrm{X}$-ray diffraction. Due to their excellent stability and high $\mu \cdot r$ value, the compounds crystals were chosen for an absorption comparison study of two different microfocus X-ray sources. ${ }^{[83]}$ In Figure 2.5, the asymmetric unit of compound $\mathbf{9}$ which crystallises in the monoclinic space group $P 2_{1} / n$ is shown. The $\mathrm{Br}-\mathrm{C}$ distances listed in Table 2.3 are slightly longer than the typical bond length of $195 \mathrm{pm}^{[84]}$ and their angles to the acridine backbone (C4-C15-Br1 and $\mathrm{C} 5-\mathrm{C} 16-\mathrm{Br} 2$, respectively) are a bit widened in comparison to the ideal tetrahedral angle. Although the structure is substituted symmetrically at the 4- and 5-positions, the torsion angle of C6-C5-C16- $\mathrm{Br} 2$ $\left(97.52(17)^{\circ}\right)$ deviates stronger from the orthogonal angle of $90^{\circ}$ than $\mathrm{C} 3-\mathrm{C} 4-\mathrm{C} 15-\mathrm{Br} 1$ ($\left.92.33(18)^{\circ}\right)$. It is, therefore, more bended towards the inner centre. Differently from expectations, both bromine side arms point to the same direction although they have a high steric demand. The reason for that can be found in the packing of the crystal which is demonstrated in Figure 2.6. The two molecules have an opposite orientation in space and are shifted sideward by one benzene unit. Hence the bromine atoms lay on the same side, the $\pi$-systems of the fluorophores could get in closer contact. The distance between two planes generated through the inner six-membered ring of the neighboured acridine derivatives is $347.3 \mathrm{pm}$. A value around $350 \mathrm{pm}$ has often been observed in publications with stacked fluorophores ${ }^{[85]}$ likewise in solid state investigations of anthracene derivatives in our working group. ${ }^{[25 \mathrm{~d}]}$ In these publications, the influence of the packing effects on solid state fluorescence is determined. However, this will not be discussed here since it was not the focus of this thesis.
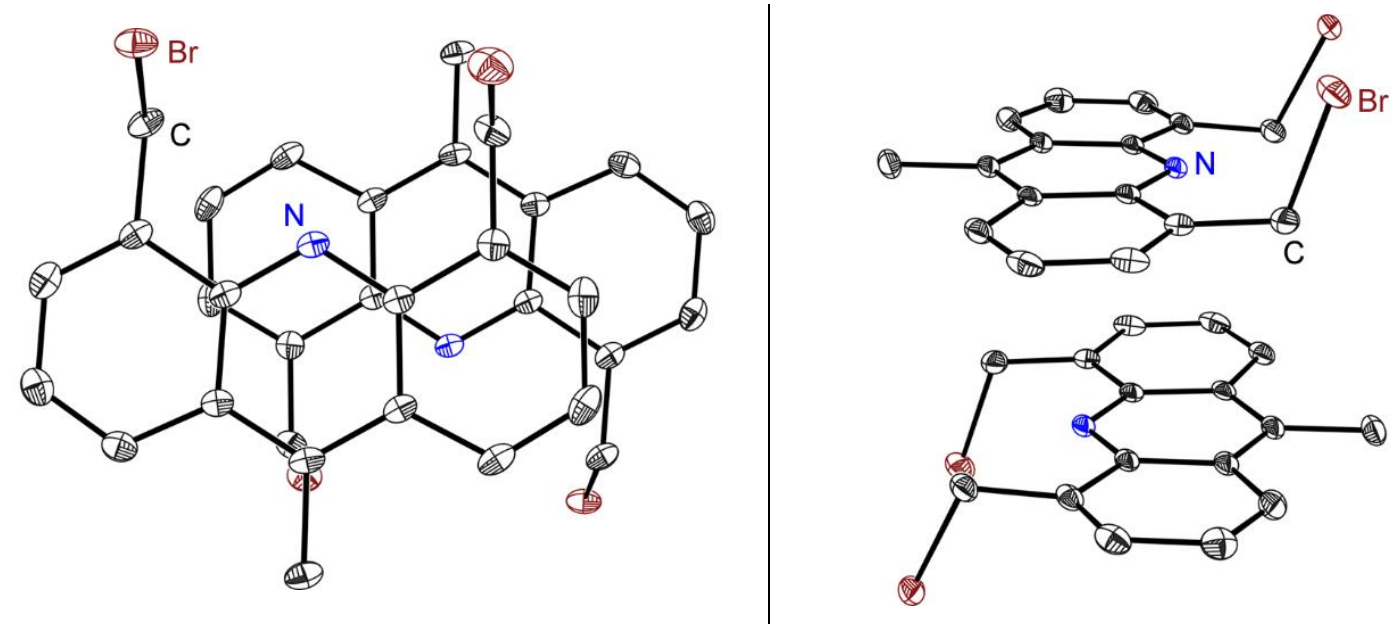

Figure 2.6: Different views of the solid state structures of 9 demonstrating the packing effect of the crystal.

At second glance at the packed structures, some interesting van der Waals interactions of the halogens have been found (Figure 2.7). The solid state structure of 9 involves $\mathrm{CH} \cdots \mathrm{Br}$ and $\mathrm{Br} \cdots \mathrm{Br}$ interactions. Especially the latter are attractive forces that have been utilised in crystal engineering. ${ }^{[86]}$ These forces are usually of weak character but they can have significant influences to the orientation of the molecules. 
(a)

$$
\begin{aligned}
& \stackrel{\mathrm{C}}{\theta_{1}} \cdot \frac{\theta_{2}}{\mathrm{x}-\mathrm{C}} \\
& \left|\theta_{1}-\theta_{2}\right| \leq 15^{\circ}
\end{aligned}
$$

(b)

$$
\underbrace{\mathrm{X}}_{\theta_{1}}-{ }_{\theta_{2}}^{-\mathrm{X}}
$$$$
30^{\circ} \leq\left|\theta_{1}-\theta_{2}\right|
$$

(c)

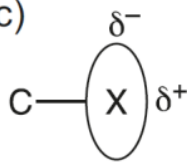

Scheme 2.13: Different kinds of halogen $\cdots$ halogen interactions. Type-I interaction (a), Type-II interaction (b), and the polar flattening effect in halogenated organic compounds (c). ${ }^{[55 d]}$

There are two different preferred geometries possible for the halogen $\cdots$ halogen contacts (Scheme 2.13). In general, these contacts are only termed as an interaction if the interhalogen distance is equal or less than the sum of the van der Waals radii $\left(r_{\mathrm{vdw}}\right)$. For two bromine atoms, $r_{\mathrm{vdw}}$ is $370 \mathrm{pm}^{[87]}$ which is the same value measured for the $\mathrm{Br} 1 \cdots \mathrm{Br} 1$ distance in 9 (370.4 pm). The angles $\theta_{1}$ and $\theta_{2}$ are equal $\left(151.9^{\circ}\right)$ due to the intermolecular contact between the same bromine atoms $(\mathrm{Br} 1)$. The resulting torsion angle of $180^{\circ}$ indicates a Type-I geometry in this case, too. These geometries result from the polar flattening effect in halogenated organic compounds which is illustrated in Scheme 2.13, c. Thus, the polarised atoms can develop attractive forces. These structural determinations are rare in the literature concerning halogen-containing acridines, ${ }^{[88]}$ whereas Nabeshima et al. ${ }^{[85 \mathrm{~d}]}$ have done similar interesting investigations to 2,7-dibromoacridine.

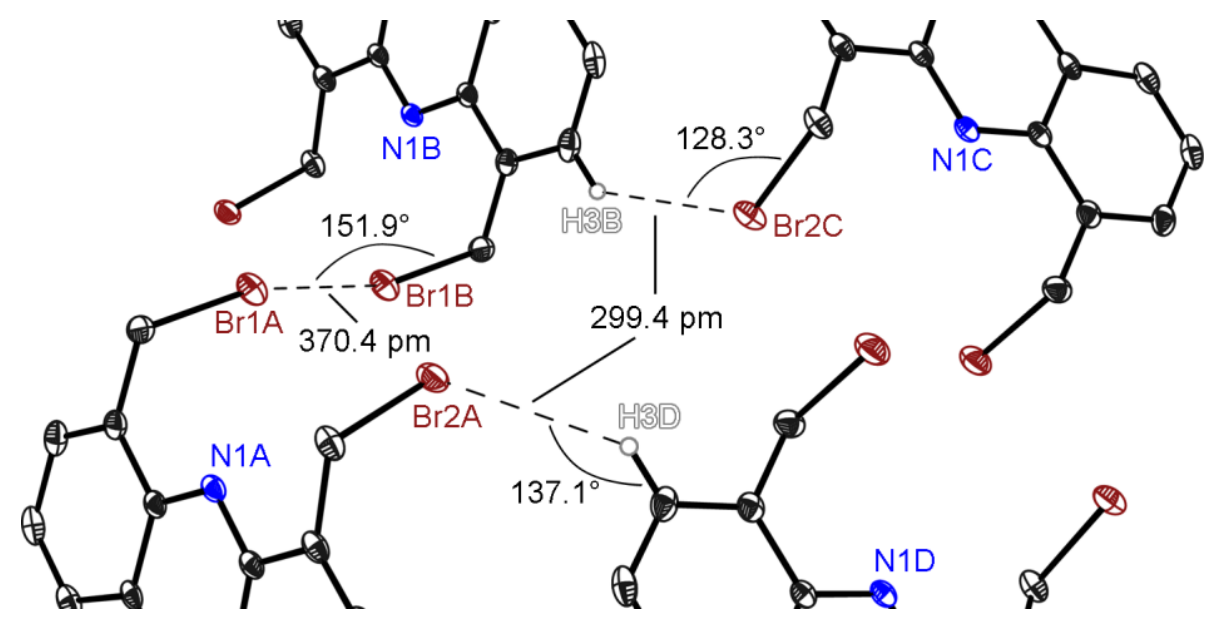

Figure 2.7: Detail of the packed cell visualising the $\mathrm{Br} \cdots \mathrm{Br}$ and $\mathrm{CH} \cdots \mathrm{Br}$ interactions.

The second halogen interaction $(\mathrm{CH} \cdots \mathrm{Br})$ can be termed either as the already discussed van der Waals force or eventually as a weak hydrogen bond. The hydrogen atom has an $r_{\mathrm{vdW}}$ value of $120 \mathrm{pm},{ }^{[87]}$ resulting in a sum of $305 \mathrm{pm}$ for $\mathrm{H}+\mathrm{Br}$. The measured distance in the solid state is $299.4 \mathrm{pm}(\mathrm{H} 3 \cdots \mathrm{Br} 2)$ which is a smaller value than the van der Waals sum. It needs to be mentioned that the hydrogen atom $\mathrm{H} 3$ is not refined freely and, therefore, its geometry properties cannot be discussed in detail. Nevertheless, the interaction with the bromine atom is obvious and the resulting values should be seen as a rough approximation. According to Steiner, ${ }^{[89]}$ even the weak $\mathrm{C}-\mathrm{H}$ donor groups are able to form "normal" hydrogen bonds. Their length should be less than $320 \mathrm{pm}$ or better $300 \mathrm{pm}$ which is the case here. The $\mathrm{C}-\mathrm{H} \cdots \mathrm{A}$ angle should be above $110^{\circ}$ for weak and above $130^{\circ}$ for moderate hydrogen bonds. Here, the $\mathrm{C}-\mathrm{H} \cdots \mathrm{Br}$ angle is $137.1^{\circ}$ and $128.3^{\circ}$ for $\mathrm{H} \cdots \mathrm{Br}-\mathrm{C}$, respectively.

The structures $\mathbf{8}$ and $\mathbf{9}$ feature the needed methylene units as spacer and, furthermore, the halides as a good leaving group. With these precursors in hand, only one more step was needed to replace the bromine atoms with amine receptor units. 


\subsubsection{4,5-Bis(di(2’-methylthiobenzyl)aminemethylene)-9-methylacridine (10)}

A lot of different amine derivatives can be chosen for the substitution of the bromines. Apart from the nitrogen atom of the amine itself, it should have more heteroatoms like nitrogen, oxygen, or sulfur atoms to improve the chelating effect. The side arms can be sterically demanding to reduce the pocket size for a possible coordinated analyte. This should enhance the selectivity of the molecular sensor which is one of its most important properties. Concerning the precursor, the receptor units were introduced in the 4- and 5-positions of the acridine system. This arrangement is a great advantage since the side arms are in close proximity to each other and, therefore, are able to coordinate to the same analyte. This can also improve the selectivity of the system. Moreover, when both amines chelate a target cation, the analyte is forced in close proximity to the centred acridine nitrogen atom which then can act as a further donor side. This principle is demonstrated in the literature ${ }^{[49,56-57]}$ and has been discussed in the introduction of this thesis. It would be of great benefit when this sparsely explored coordination motif would lead to a significant shift of the emission wavelength, resulting in a colorimetric response. However, the researchers investigating this phenomenon so far only reported bathochromic shifts of $16^{[49]}$ to $19 \mathrm{~nm}^{[90]}$. The investigation of this possible coordination of the aromatic nitrogen atom to an analyte was one aim of this thesis.

In the following synthesis, each amine receptor is bonded to two benzyl groups bearing methylthio functionalities (Scheme 2.14). The benzyl groups provide the steric demand but can freely rotate due to their methylene bridge. The four sulfur atoms are able to coordinate an analyte, especially large and soft metal ions concerning to the hard and soft (Lewis) acids and bases concept (HSAB). ${ }^{[91]}$
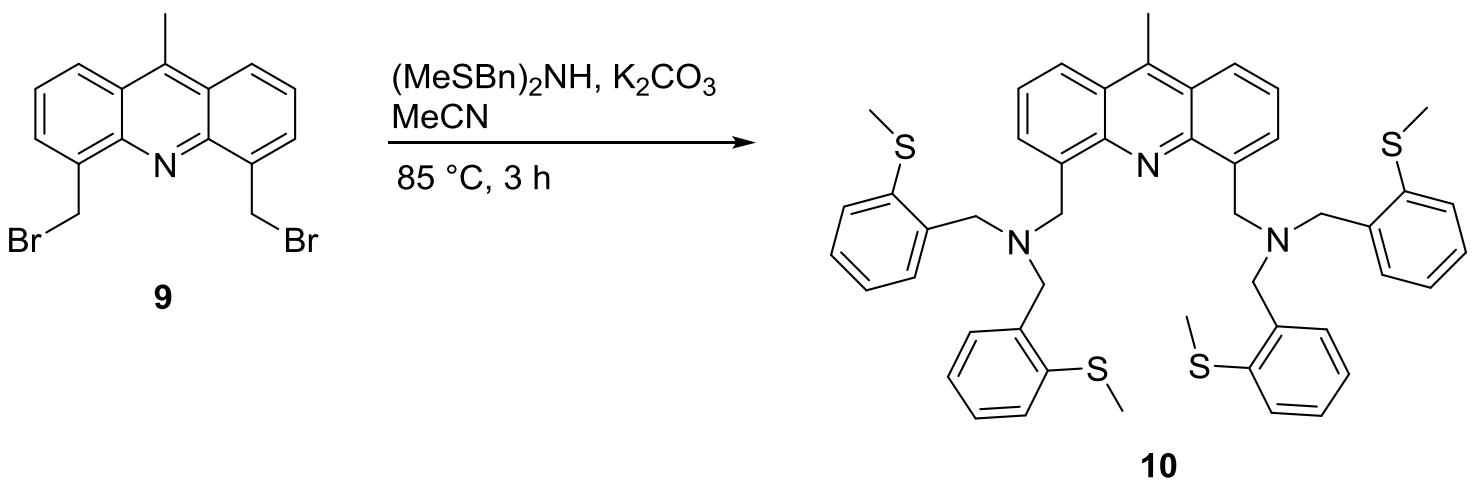

Scheme 2.14: Synthesis of 10 by a substitution reaction of 9 with bis(2-methylthiobenzyl)amine.

The bis(2-methylthiobenzyl)amine was synthesised by Finkelmeier during his $\mathrm{PhD}$ thesis in the Stalke group. ${ }^{[25 \mathrm{~d}]}$ For the synthesis of $\mathbf{1 0}$, two equivalents of the amine were given to a suspension of 9 and an excess of $\mathrm{K}_{2} \mathrm{CO}_{3}$ in acetonitrile. The oil bath was heated up to $90{ }^{\circ} \mathrm{C}$ for three hours. During the reaction, a brown solid was formed which stuck at the surface of the flask. Therefore, the solvent was decanted after cooling to room temperature and the precipitate was washed inside the flask with hexane. The crude product was dissolved in toluene whereat the insoluble inorganic salts were filtered off. After the removal of the solvent under reduced pressure, compound $\mathbf{1 0}$ was almost pure with a yield of $48 \%$, but for fluorescence spectroscopy analysis it needs to be highly purified. It was tried to separate the small amounts of side products with the help of column 
chromatography $\left(\mathrm{Al}_{2} \mathrm{O}_{3}\right.$, PE/EA, 80:1 $\left.\rightarrow 1: 20\right)$ but only mixed fractions were mainly obtained. In a small amount of test tubes, the purified product could be identified. Small crystals, suitable for $X$-ray structure determination, were isolated from these tubes (Figure 2.8). 10 crystallises in the triclinic space group $P \overline{1}$ with one molecule in the asymmetric unit. The nitrogen atoms of the amines exhibit a tetragonal environment due to their $\mathrm{sp}^{3}$ hybridisation with an angular sum of $328.88^{\circ}(\mathrm{N} 2)$ and $329.02^{\circ}(\mathrm{N} 3)$, respectively. The ideal angular sum is $322.41^{\circ}$. Selected bond lengths and angles of the structure are listed in Table 2.4.

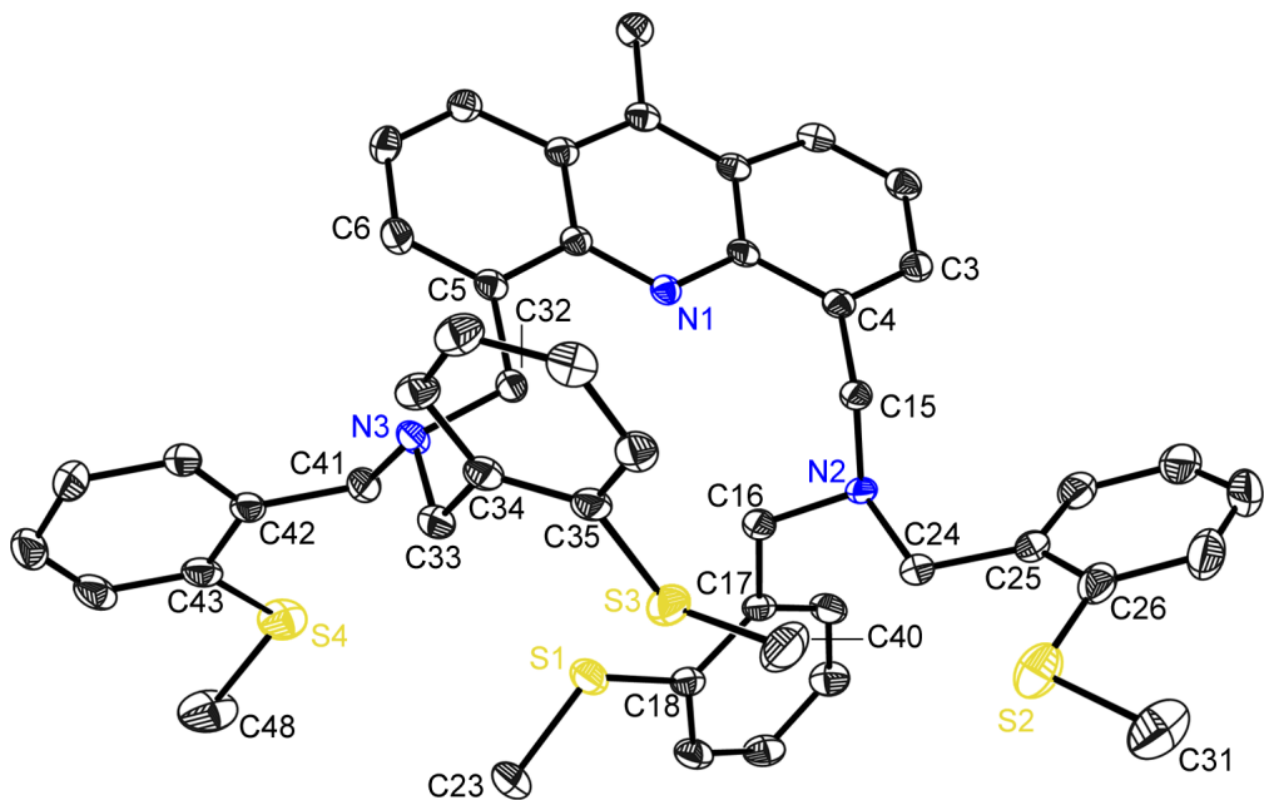

Figure 2.8: Solid state structure of $\mathbf{1 0}$. Hydrogen atoms are omitted for the sake of clarity.

The torsion angles of the methylene bridged amine units vary considerably. The receptor groups at the 4- and 5-position are chemically equivalent, but due to their huge steric demand they turn in different directions. The torsion angle of $\mathrm{C} 3-\mathrm{C} 4-\mathrm{C} 15-\mathrm{N} 2$ with a value of $76.7^{\circ}$ illustrates the rotation of the amine $\mathrm{N} 2$ more towards a perpendicular arrangement to the aromatic plane. In comparison to that, the amine $\mathrm{N} 3$ lies almost in the acridine plane, with an angle of $11.5^{\circ}$ (C6C5-C32-N3).

Table 2.4: Selected bond lengths and angles of $\mathbf{1 0}$.

\begin{tabular}{cc|cc|cc}
\hline Bond [pm] & & Angle $\left[^{\circ}\right]$ & & Torsion angle $\left[^{\circ}\right]$ & \\
\hline \hline N2-C15 & $148.1(2)$ & C4-C15-N2 & $113.34(14)$ & C3-C4-C15-N2 & $-76.7(2)$ \\
N2-C16 & $147.0(2)$ & C15-N2-C16 & $109.48(13)$ & C6-C5-C32-N3 & $11.5(2)$ \\
N2-C24 & $146.6(2)$ & C5-C32-N3 & $112.65(14)$ & C15-N2-C16-C17 & $171.38(14)$ \\
N3-C32 & $146.5(2)$ & C32-N3-C33 & $110.03(13)$ & C15-N2-C24-C25 & $-70.98(18)$ \\
N3-C33 & $147.1(2)$ & C18-S1-C23 & $103.30(10)$ & C32-N3-C33-C34 & $64.75(19)$ \\
N3-C41 & $146.7(2)$ & C26-S2-C31 & $103.27(12)$ & C32-N3-C41-C42 & $-159.72(14)$ \\
S1-C18 & $177.2(2)$ & C35-S3-C40 & $103.07(10)$ & C23-S1-C18-C17 & $176.43(15)$ \\
S1-C23 & $180.2(2)$ & C43-S4-C48 & $104.04(12)$ & C31-S2-C26-C25 & $167.30(15)$ \\
S4-C43 & $176.5(2)$ & & & C40-S3-C35-C34 & $-166.25(15)$ \\
S4-C48 & $179.5(2)$ & & & C48-S1-C43-C42 & $168.05(15)$ \\
\hline
\end{tabular}


Nevertheless, both receptors show to the same side of the aromatic structure, which is demonstrated in Figure 2.9, left. The $\mathrm{N}-\mathrm{C}$ distances are in good agreement with typical bond length for $\mathrm{sp}^{3}$ hybridised atoms $(147 \mathrm{pm})$. The same is true for the $\mathrm{S}-\mathrm{C}\left(\mathrm{sp}^{2}\right)$ bond lengths whereas the $\mathrm{S}-\mathrm{C}\left(\mathrm{sp}^{3}\right)$ distances are all shortened (typical values are $176 \mathrm{pm}$ and $183 \mathrm{pm}$, respectively). ${ }^{[84]}$ In Table 2.4, the longest (S1) and shortest (S4) values are listed for the sulfur environments. The $\mathrm{C}-\mathrm{S}-\mathrm{C}$ angles are quite similar and all smaller than the ideal tetrahedral angle due to the strong steric demand of the two lone pairs at each sulfur atom. Additionally, all torsion angles containing the sulfur and nitrogen atom are listed to visualise the difference of the geometry, especially in the case of the amines.
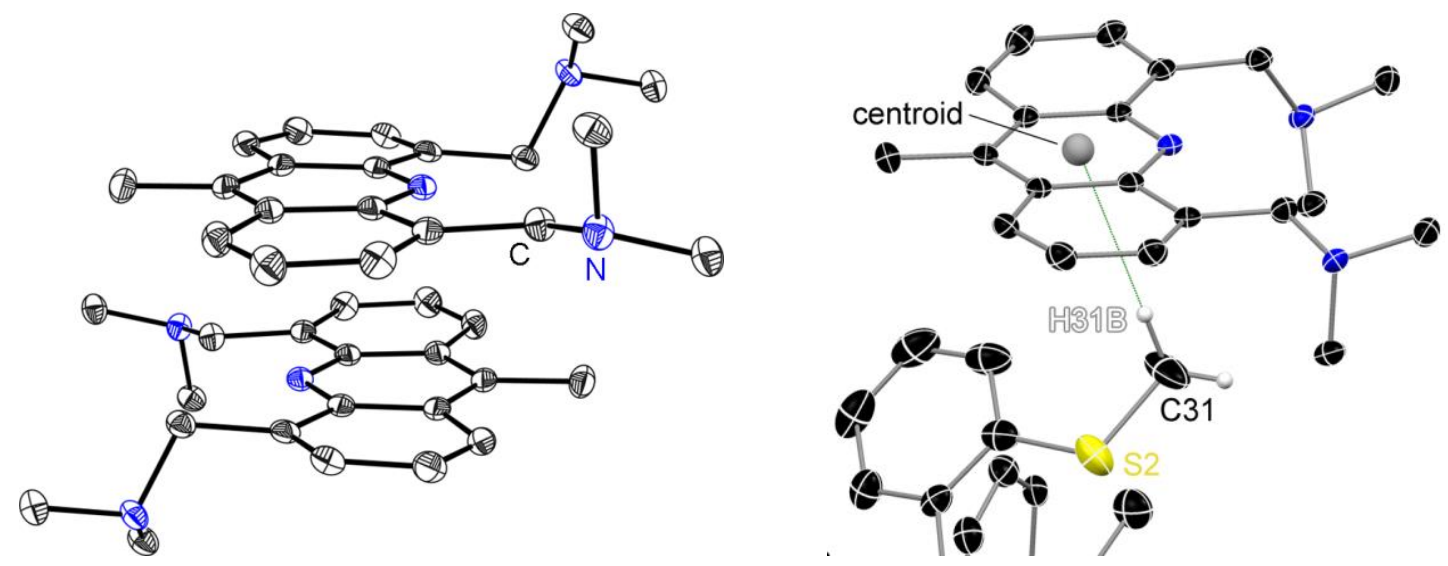

Figure 2.9: Left: side view of the packing effect of the acridine systems in 10; right: visualising the intermolecular $\mathrm{CH} \cdots \pi$-interaction. The positions of the hydrogen atoms at $\mathrm{C} 31$ were refined freely for the discussion.

The solid state structure of the precursor 9 showed a packing effect of the acridine backbone. The same is true for this structure, demonstrated in Figure 2.9, left. The distance between the two aromatic $\pi$-systems is $339.6 \mathrm{pm}$, an even smaller value than for 9 (347.3 pm). Likewise, the acridine units are pointing in opposite directions, however, they are less shifted resulting in a higher overlap. This could explain the stronger attraction. Furthermore, one of the phenyl rings bonded to C41 shows an additional overlap to its next counterpart (Figure 2.10) with a distance of $356.5 \mathrm{pm}$.

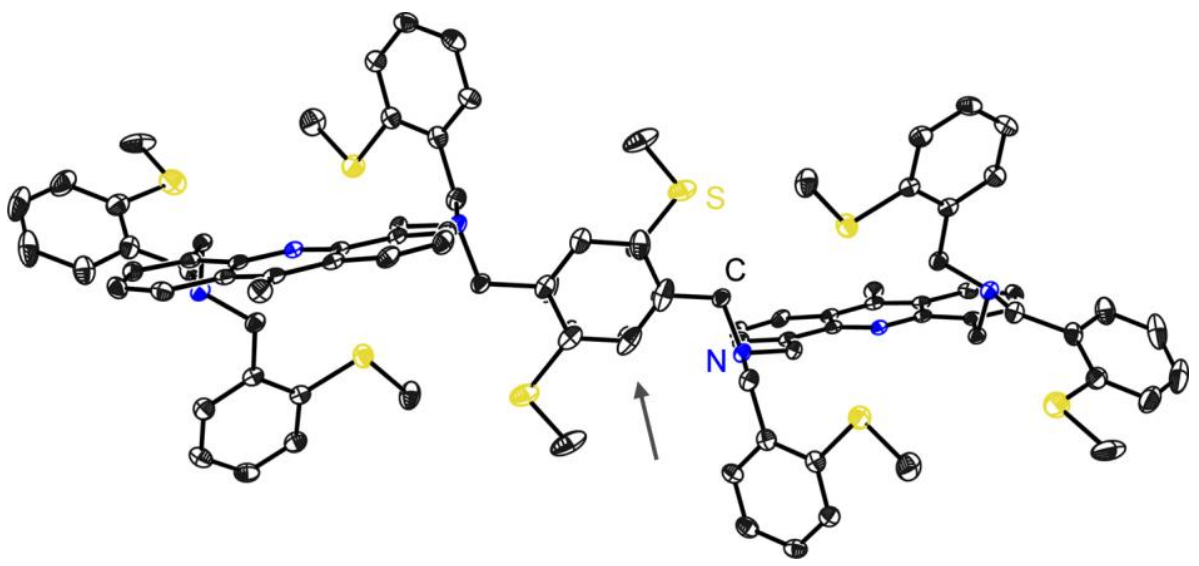

Figure 2.10: $\pi$ - $\pi$-overlap of two phenyl groups marked with an arrow. 
Another interaction which stabilises the geometry of the solid state structure is an intermolecular $\mathrm{CH} \cdots \pi$-interaction of a hydrogen to the centre of an acridine ring. The hydrogen atom (H31B) is linked to the methyl group of S2. To determine the strength of this interaction, a centroid was calculated in the centre of the inner six-membered ring of acridine. The distance between H31B and the centroid is 283(2) pm and 258(2) pm orthogonal to the aromatic plane. The distances are in line with the averaged literature value of 272(18) pm, calculated by 9520 entries in the CSD database with $\mathrm{CH} \cdots \pi$ interactions. ${ }^{[92]}$ The $\mathrm{CH} \cdots \pi$ angle to the centroid is $175(2)^{\circ}$. This angle is nearly linear indicating a strong interaction for this type of phenomenon.

The pureness of the single crystals obtained from the column chromatography allowed analysing the compound by fluorescence spectroscopy.

\subsubsection{Luminescence procedure and sample preparation}

Before realising the fluorescence measurements, several important issues have to be considered carefully. At first, a series of common errors in sample preparation are possible. The compound to be analysed has to be absolutely pure since small impurities can have a significant effect on the fluorescence spectra. An aromatic side product can overlap with the actual emission spectrum and alters the curve. Additionally, an incorrect excitation wavelength could be used due to a wrongly assigned spectrum. That is why it is useful to record spectra at different excitation wavelengths to determine if the emission spectrum remains the same. This should be the case since emission spectra are usually independent of the incident light. ${ }^{[93]}$

For appropriate measurements, the correct concentration level of the samples needs to be set. On the one hand, a strongly diluted sample can lose too much intensity which results in a bad signalto-noise ratio. On the other hand, too strong concentrated samples are sources of several

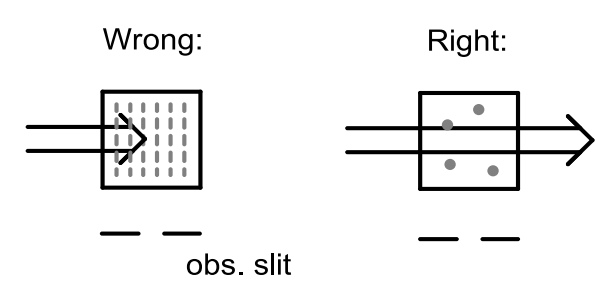

Figure 2.11: Absorption of the incident beam at highly concentrated samples (left). significant errors. The absorption of the fluorophore leads to a reduction of the emitted light at too high concentrations (Figure 2.11), which is termed the inner filter effect. Furthermore, a large amount of molecules results in a higher number of collisions of excited fluorophores. By that, the absorbed energy decays in a non-radiative pathway whereby the molecules return to their ground state. ${ }^{[7]}$ This supresses the actual intensity of the measured sample.

Some fluorophores like pyrene show another concentration-dependent behaviour. If sufficient molecules are present in solution, they can form excited-state dimers, called 'excimer' ${ }^{[19,94]}$ Thus, new excimer peaks at higher wavelength show up at the emission spectrum. This topic will be discussed more intense in chapter 2.2.11.

The right range of concentration needs to be determined experimentally by titration of the analyte with the used solvent. These dilution experiments have already been carried out in the master thesis with comparable compounds. ${ }^{[63]}$ The resultant concentration ranges differ for each fluorophore, which is why the low concentration of $1.0 \cdot 10^{-5} \mathrm{M}$ was chosen. At this value, the intensity decreases in linear relation with the decrease of concentration. 
The next important issue was the choice of the solvent. It is essential to have completely clear solutions without any precipitates. Therefore, one demand to the solvent is the ability to dissolve organic compounds (molecular sensor) as well as inorganic metal salts (target ions). Otherwise, the used concentrations would differ in the experiment or remaining solids could scatter the incident beam, distorting the results. A second demand deals with the possibility to use a molecular sensor in medicinal applications. For this purpose, e.g. the detection of metal ions in blood, the sensor should be able to operate in water. Hence, this is a very ambitious goal and causes more challenges which will be discussed later on in this thesis (Chapter 2.2.14). A good alternative to that is the polar protic solvent methanol. It is able to dissolve most organic compounds and a wide range of metal salts. For a good comparison of the spectroscopic results, every tested metal ion should have the same counter anion. It should not be able to strongly complex the cations like acetate or carbonate ions, avoiding a competition with the added sensor. Available for a wide range of metal salts and soluble in methanol are the metal bromides and, therefore, mainly used in this thesis.

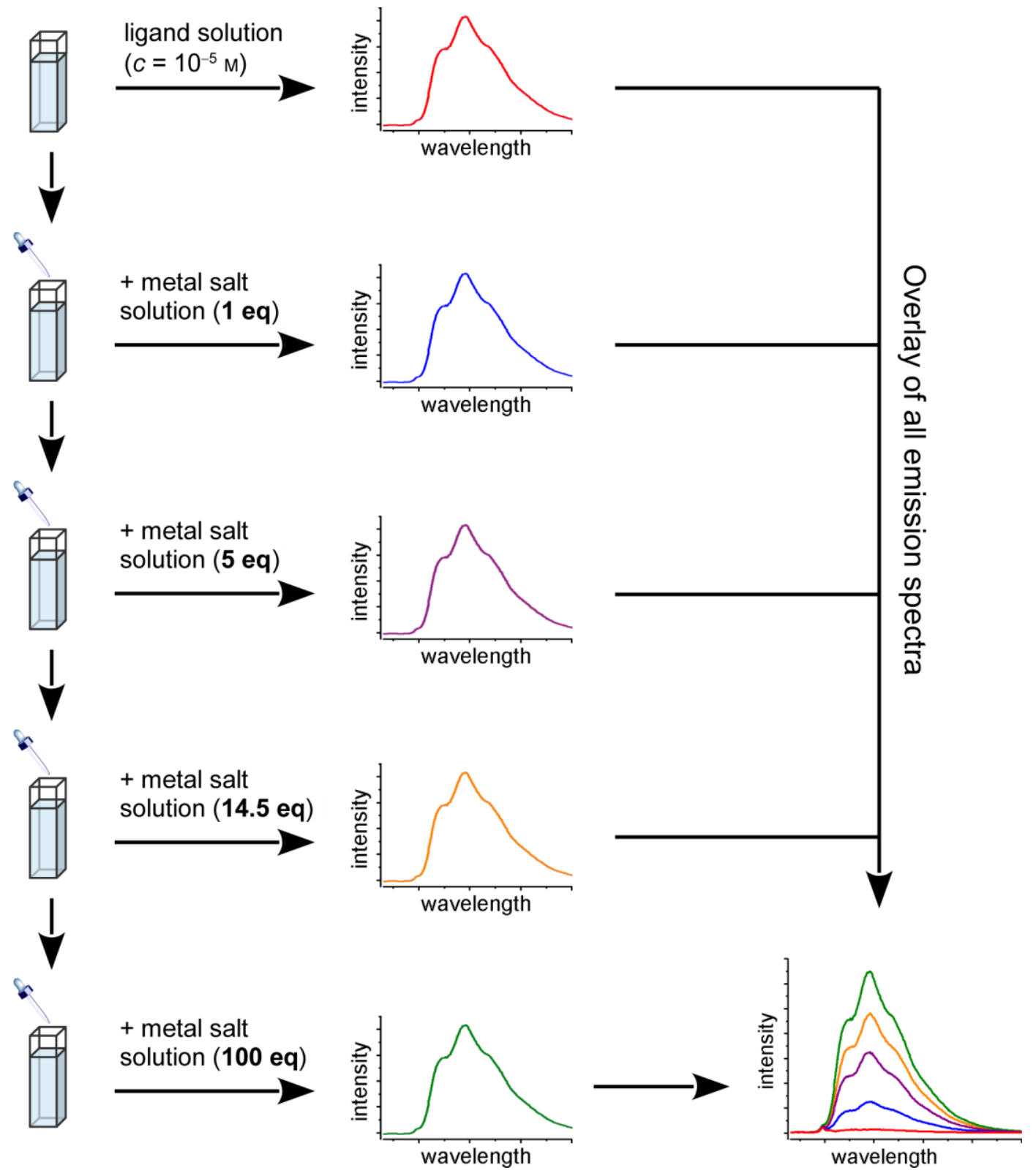

Figure 2.12: Screening procedure for metal ions used in this thesis. 
The synthesised compounds with the "fluorophore-spacer-receptor" structure had to be tested for their possibility to work as a molecular sensor for metal cations. To get a wide and quick overview if the addition of an analyte alters the emission spectrum of a sensor system, a screening procedure was established (Figure 2.12). Sixteen different metal ions were chosen across the periods of elements (Figure 2.13). Each was added as a solution to the dissolved ligand in four steps in a range from 1 to 100 equivalents. If any effect was observed, a more intense study of this analyte was performed.

\begin{tabular}{|c|c|c|c|c|c|c|c|c|c|c|c|c|c|c|c|c|c|}
\hline 1 & & & & & & & & & & & & & & & & & 18 \\
\hline $\mathrm{H}$ & 2 & & & & & & & & & & & 13 & 14 & 15 & 16 & 17 & $\mathrm{He}$ \\
\hline $\mathrm{Li}$ & $\mathrm{Be}$ & & & & & & & & & & & B & C & $\mathrm{N}$ & $\mathrm{O}$ & $\mathrm{F}$ & $\mathrm{Ne}$ \\
\hline $\mathrm{Na}$ & $\mathrm{Mg}$ & 3 & 4 & 5 & 6 & 7 & 8 & 9 & 10 & 11 & 12 & $\mathrm{Al}$ & $\mathrm{Si}$ & $\mathrm{P}$ & $\mathrm{S}$ & $\mathrm{Cl}$ & $\mathrm{Ar}$ \\
\hline $\mathrm{K}$ & $\mathrm{Ca}$ & $\mathrm{Sc}$ & $\mathrm{Ti}$ & $\mathrm{V}$ & $\mathrm{Cr}$ & $\mathrm{Mn}$ & $\mathrm{Fe}$ & Co & $\mathrm{Ni}$ & $\mathrm{Cu}$ & $\mathrm{Zn}$ & $\mathrm{Ga}$ & $\mathrm{Ge}$ & As & $\mathrm{Se}$ & $\mathrm{Br}$ & $\mathrm{Kr}$ \\
\hline $\mathrm{Rb}$ & $\mathrm{Sr}$ & $\mathrm{Y}$ & $\mathrm{Zr}$ & $\mathrm{Nb}$ & Mo & $\mathrm{Tc}$ & $\mathrm{Ru}$ & $\mathrm{Rh}$ & $\mathrm{Pd}$ & $\mathrm{Ag}$ & $\mathrm{Cd}$ & In & $\mathrm{Sn}$ & $\mathrm{Sb}$ & $\mathrm{Te}$ & I & $\mathrm{Xe}$ \\
\hline Cs & $\mathrm{Ba}$ & $\mathrm{La}$ & $\mathrm{Hf}$ & $\mathrm{Ta}$ & $\mathrm{W}$ & $\mathrm{Re}$ & Os & $\mathrm{Ir}$ & $\mathrm{Pt}$ & $\mathrm{Au}$ & $\mathrm{Hg}$ & $\mathrm{Tl}$ & $\mathrm{Pb}$ & $\mathrm{Bi}$ & Po & At & $\mathrm{Rn}$ \\
\hline
\end{tabular}

Figure 2.13: Periodic table of the chemical elements. Metalloids and non-metallic elements are greyshaded. The utilised metal ions for the screening procedure are marked in green.

The dissolving of the metal salts was carried out with the same solvent as for the ligand, unless it was stated otherwise. As described before, the most frequently used solvent was methanol, but in some cases tetrahydrofuran, dichloromethane, or even water were used, too. To be able to add an equivalent range from 1 to 100 into the small cuvettes, two different concentrated solutions of the metal salts were prepared (Table 2.5). The quantities shown in the legend of the fluorescence spectra are added up values (from bottom to top). This is valid for all experiments discussed in this thesis.

Table 2.5: Used concentrations for the spectroscopic investigations.

\begin{tabular}{cc|cccc}
\hline & ligand & salt: $\mathbf{1}$ eq & $\mathbf{5 ~ e q}$ & $\mathbf{1 4 . 5} \mathbf{~ e q}$ & $\mathbf{1 0 0} \mathbf{~ e q}$ \\
\hline \hline$c[\mathrm{~mol} / \mathrm{l}]$ & $1 \cdot 10^{-5}$ & $2.4 \cdot 10^{-4}$ & $2.4 \cdot 10^{-4}$ & $2.28 \cdot 10^{-3}$ & $2.28 \cdot 10^{-3}$ \\
$V[\mathrm{ml}]$ & 2.4 & 0.1 & 0.4 & 0.1 & 0.9 \\
\hline
\end{tabular}

\subsubsection{Luminescence properties of 4,5-bis(di(2'-methylthiobenzyl)aminemethylene)- 9-methylacridine (10)}

The first two luminescent experiments which should be carried out with a new compound are the recordings of an excitation and an emission spectrum. For the excitation spectrum, the detector is set to a specific emission wavelength whereas the sample solution is irradiated at varied energy of light. From this spectrum, the information for the setting of the proper wavelength for the record of the emission spectrum can be gained. In this case, the sample is irradiated at the defined wavelength and the detector records the emitted light.

In the previous chapter, methanol was described as the solvent of choice for the metal ion screening. In this case, acetonitrile was used to dissolve the metal salts and, due to a better solubility, tetrahydrofuran for the ligand. These solvents form less strong solvent shells around the metal ions in comparison to methanol. Otherwise, the high polar methanol would be in strong competition to the amine groups of the chemical sensor. Since in this case only $1.4 \mathrm{mg}$ of purified crystalline material of $\mathbf{1 0}$ could be isolated, only a few spectroscopic experiments could be 
performed. With this amount, an overall sample volume of $35 \mathrm{ml}$ was obtained with a concentration of $10^{-5} \mathrm{M}$. That is why the conflict with a too polar solvent should be reduced. THF was chosen because of its less polarity in comparison to methanol and it readily dissolves the ligand.

The excitation and especially the emission spectrum of 10, depicted on the left side of Figure 2.14, show a strongly broadened band. These unstructured curves can appear when only small energy distances are present between the vibrational states which cannot be resolved by the spectrophotometer.
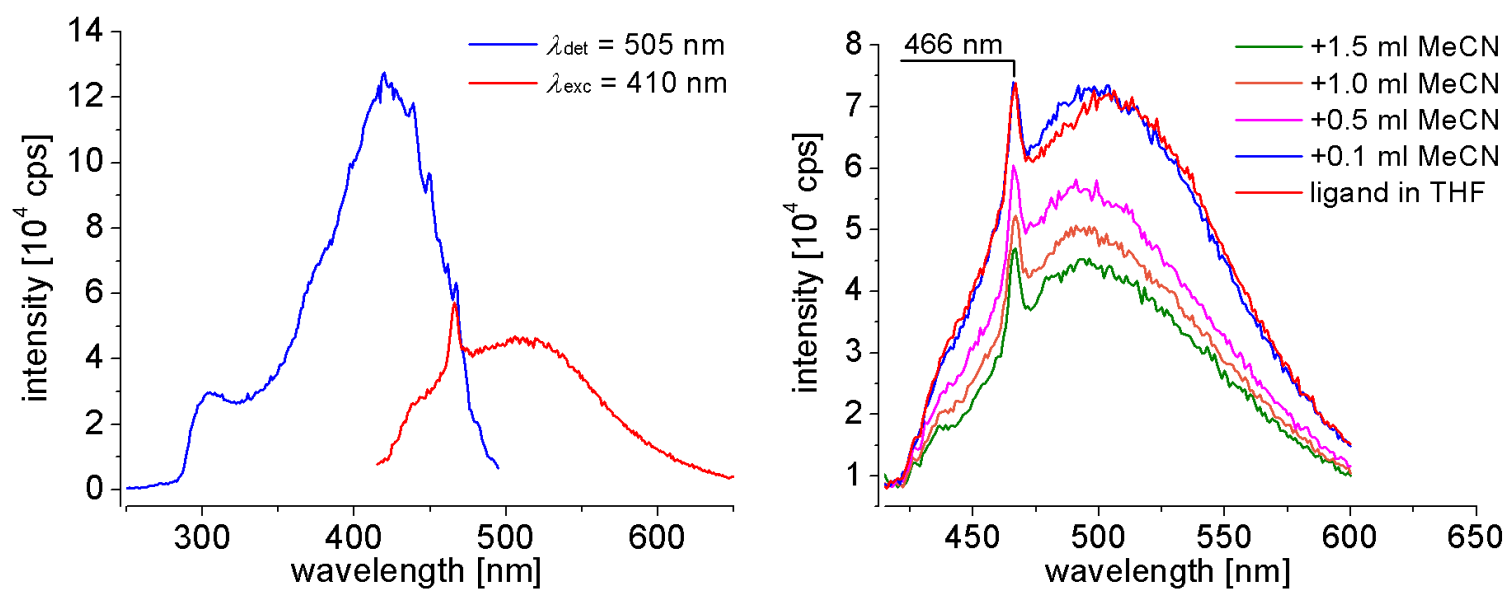

Figure 2.14: Left: Excitation (blue: $\lambda_{\text {det }}=505 \mathrm{~nm}$ ) and emission (red: $\lambda_{\mathrm{exc}}=410 \mathrm{~nm}$ ) spectrum of $\mathbf{1 0}$ in THF $\left(10^{-5} \mathrm{M}\right)$; right: emission spectra of $\mathbf{1 0}$ in THF with addition of acetonitrile $\left(\lambda_{\mathrm{exc}}=410 \mathrm{~nm}\right)$.

In comparison to the luminescence properties of pure acridine (Figure 2.15, left), the emission intensity is lowered by a factor of 11, which hints to a working PET effect. Furthermore, the emission band is shifted to longer wavelengths (bathochromic shift) for about $100 \mathrm{~nm}$. Thus, it lies more in the visible region which makes it easier to detect the altering of the emission intensity by the naked eye.

The noticeable peak at $466 \mathrm{~nm}$ is a result of Raman scattering of the used solvent tetrahydrofuran. On the one hand, these peaks can be identified by comparison with literature data, ${ }^{[95]}$ on the other hand, by varying the excitation wavelength. Signals due to Raman scattering move their position in dependency of the incident light, in contrary to the emission of the fluorophore.

On the right side of Figure 2.14, emission spectra are depicted of a THF solution of $\mathbf{1 0}$ while titrated with acetonitrile. The added volumes are equal to the titration of the metal ions. Thereby, the altering of the emission intensity by dilution can be examined as well as possible changes by mixing two different solvents. Especially in the case of charged compounds or with the presence of strong dipoles, the polarity of the solvent can significantly influence the emission wavelength. Here, only the expected decrease of intensity was observed by reducing the sample concentration.

In the next experiment, it was tested if the PET effect is working at all. As already mentioned, the first indication is the low emission intensity of the ligand in comparison to pure acridine. To check if the fluorescence can be switched back on, the lone pairs of the nitrogen atoms were forced in a bonding situation to another atom. An appropriate way for this is the protonation of the amines with an acid. Therefore, two different concentrated solutions of diluted hydrochloric acid 
were prepared $(0.001 \mathrm{M}$ and $1 \mathrm{M})$ and were added dropwise to the ligand solution (Figure 2.15, right). One drop out of a glass pipette has an approximate volume of $25 \mu \mathrm{l}$ which was determined experimentally. Nevertheless, the indications of equivalents in the legend are rounded values.

Regarding the emission spectra, there is a noticeable decrease of intensity by adding the first two amounts of $\mathrm{HCl}(0.001 \mathrm{M})$. After changing to $1 \mathrm{M}$ concentrated acid, the emission intensity rises, but only slightly higher than the beginning value. This could assume a poor working PET effect but the strong hypsochromic shift of the wavelength needs to be taken into account.
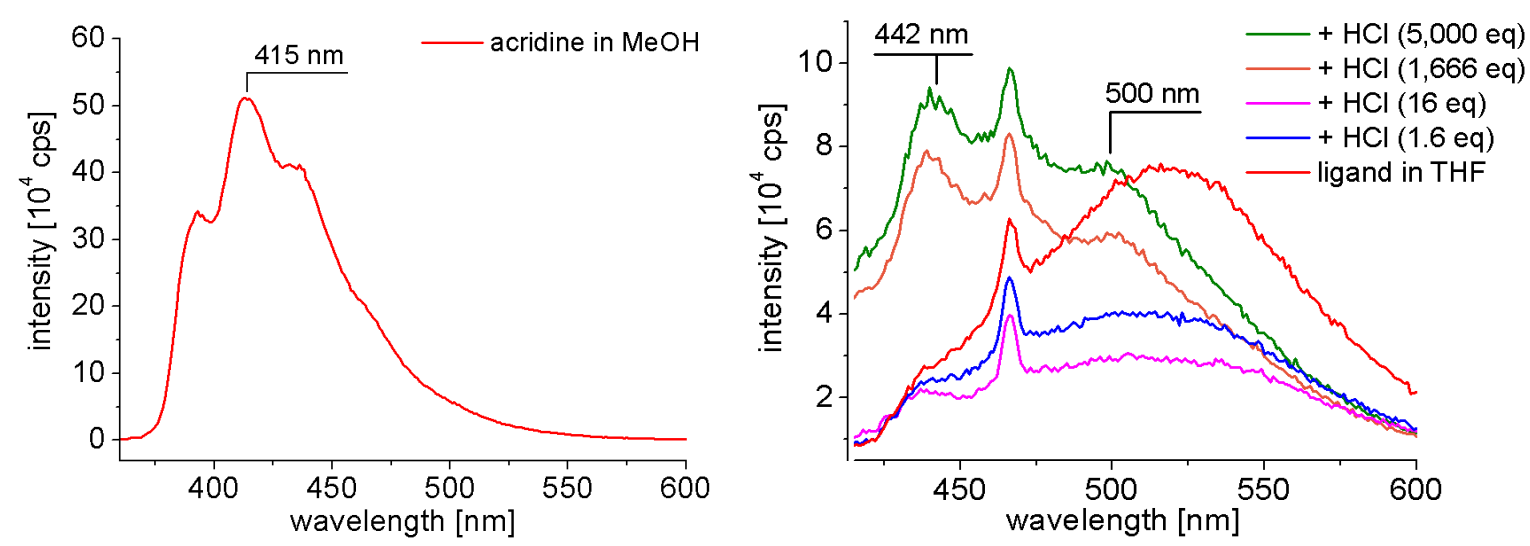

Figure 2.15: Left: Emission spectrum of acridine in methanol $\left(10^{-5} \mathrm{M}, \lambda_{\mathrm{exc}}=353 \mathrm{~nm}\right)$; right: Emission spectra of $\mathbf{1 0}$ in THF $\left(10^{-5} \mathrm{M}\right)$ titrated with aqueous hydrochloric acid at one defined wavelength $\left(\lambda_{\mathrm{exc}}=410 \mathrm{~nm}\right)$.

The spectra were recorded at a defined excitation wavelength of $410 \mathrm{~nm}$, according to the excitation spectrum of the pure ligand (Figure 2.14, left). If the excitation spectrum is altered significantly by the addition of acid, the wavelength needs to be adjusted. What can be seen in the right spectrum of Figure 2.15 is a rising new band at $442 \mathrm{~nm}$. This wavelength was chosen for recording a second excitation spectrum and is depicted in Figure 2.16 (left, green curve) in comparison to the wavelength used before (blue curve). Both spectra were recorded with an excess of $\mathrm{HCl}$.
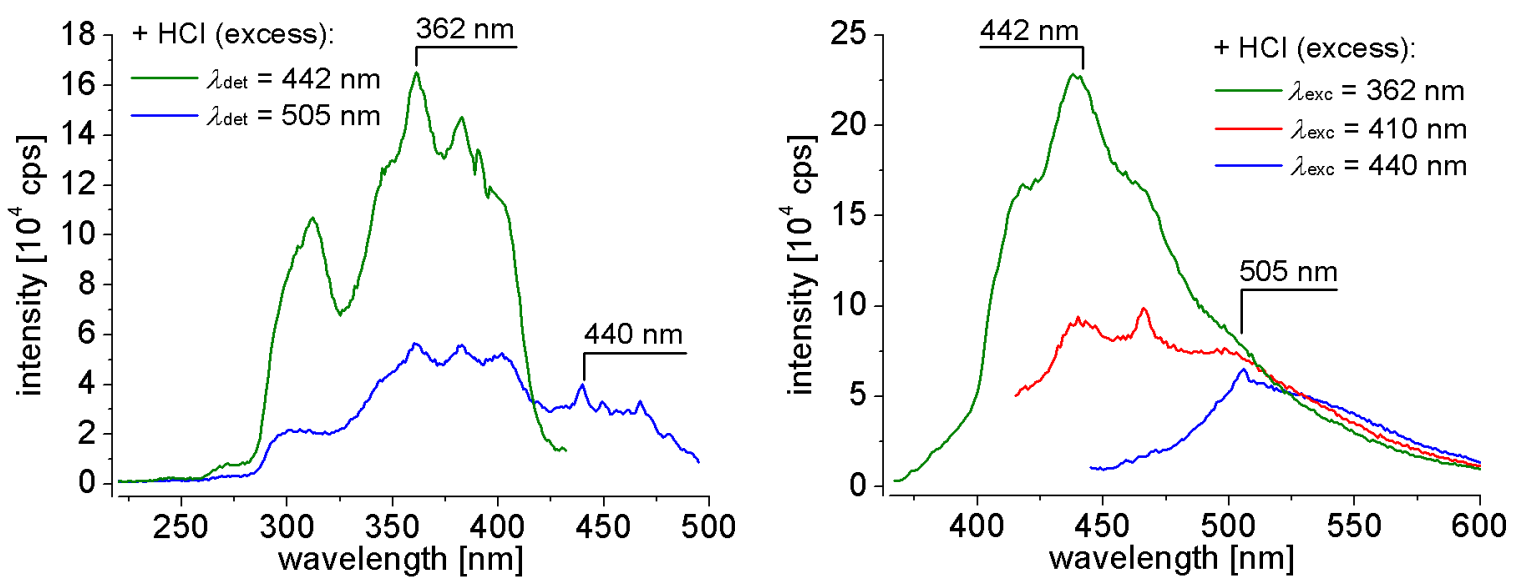

Figure 2.16: Excitation spectra (left: green: $\lambda_{\text {det }}=442 \mathrm{~nm}$, blue: $505 \mathrm{~nm}$ ) and emission spectra (right: green: $\lambda_{\text {exc }}=362 \mathrm{~nm}$, red: $410 \mathrm{~nm}$, blue: $440 \mathrm{~nm}$ ), measured at different wavelengths after addition of aqueous $\mathrm{HCl}$ to $\mathbf{1 0}$ in $\operatorname{THF}\left(10^{-5} \mathrm{M}\right)$. 
On the basis of this new spectrum, the more appropriate excitation wavelength of $362 \mathrm{~nm}$ was adjusted and compared with emission spectra at different wavelengths (Figure 2.16, right). Now, the emission curve has a well-defined shape with a strong intensity enhancement as expected for the protonation of the amine receptors.

As mentioned before, the amount of sample solution was only sufficient for several investigations. That is why the two heavy metal salts cadmium and mercury bromide were chosen which are classified as soft metal ions according to the HSAB concept of Pearson. ${ }^{[91]}$ The sulfurous receptor units of the ligand should rather be able to coordinate to these analytes.
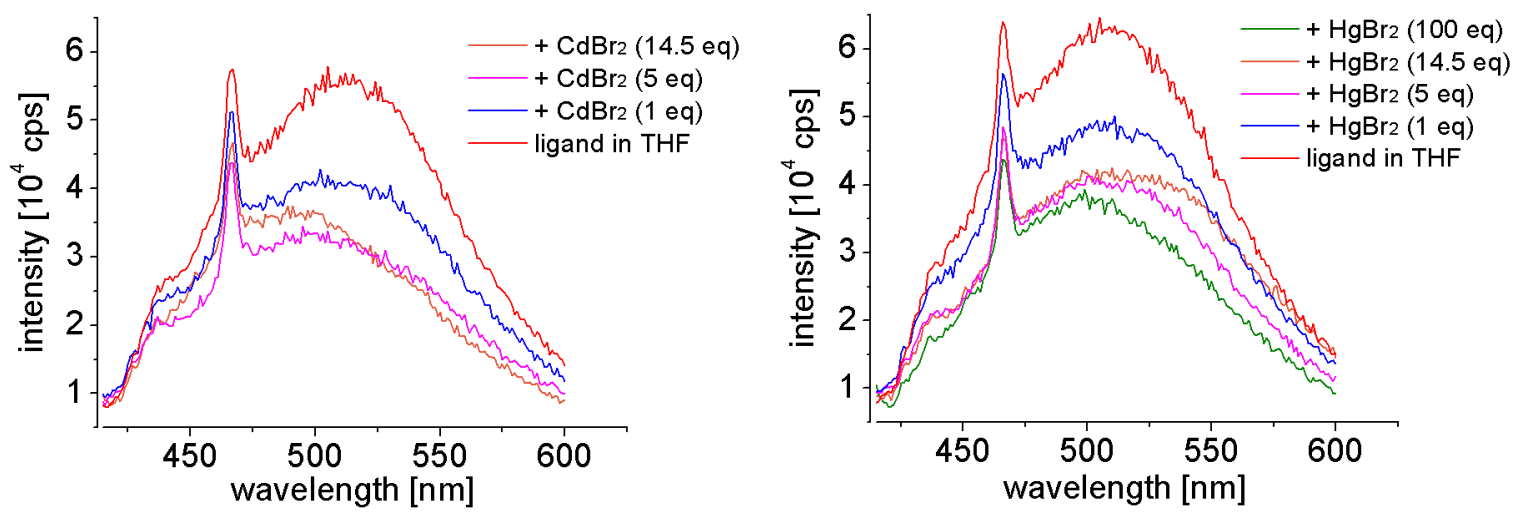

Figure 2.17: Emission spectra of $\mathbf{1 0}$ in $\operatorname{THF}\left(10^{-5} \mathrm{M}, \lambda_{\text {exc }}=410 \mathrm{~nm}\right)$ with addition of $\mathrm{CdBr}_{2}$ (left), respectively $\mathrm{HgBr}_{2}$ (right); both metal salts were dissolved in $\mathrm{MeCN}$.

Unfortunately, the titration of $\mathrm{Cd}^{2+}$ and $\mathrm{Hg}^{2+}$ solutions in $\mathrm{MeCN}$ did not alter the emission spectrum of 10 (Figure 2.17). The decrease of intensity can directly be attributed to the dilution effect shown in Figure 2.14, right. In the case of $\mathrm{CdBr}_{2}$, only a maximum of 14.5 eq could be added to the sample cuvette, due to the worse solubility of the salt in MeCN. Hence, there is no indication that the addition of a larger amount of metal ion would change the spectra significantly. Having a closer look at the receptor side arms, the assumption could be made that the methylthio benzyl groups could coordinate metal ions outside the range of the amines. If they do not coordinate to the analyte, no molecular response can be observed in the emission spectrum (onswitch) since they are responsible for the PET effect. To get an idea about what kind of structure could get formed by adding a metal ion to compound 10, the computer program Avogadro v1.1.1 was used. ${ }^{[96]}$ The $x, y, z-f i l e$ of the solid state structure was imported with the software and the geometry was optimised using the universal force field (UFF). After a cadmium ion was added close to the side arms, the geometry was optimised again. (Figure 2.18). In the case of mercury, similar observations have been made and are not shown here. First of all, it needs to be clarified that the geometry optimisation by the computer program does not necessarily reflect the solid state structure or even the conformation in solution. Hence, it can give a hint to a possible interaction of the ligand with metal ions. It is interesting to see that the cadmium ion is arranged between two of the four sulfur atoms, possibly interacting with their lone pairs. The other sulfur atoms are not able to rotate around their $\mathrm{S}-\mathrm{C}$ axis to coordinate to the metal ion since the phenyl rings would otherwise get in close contact to other groups. The coordination sphere of the $\mathrm{Cd}^{2+}$ ion is filled with at least three hydrogen bond interactions, which are not shown here. 


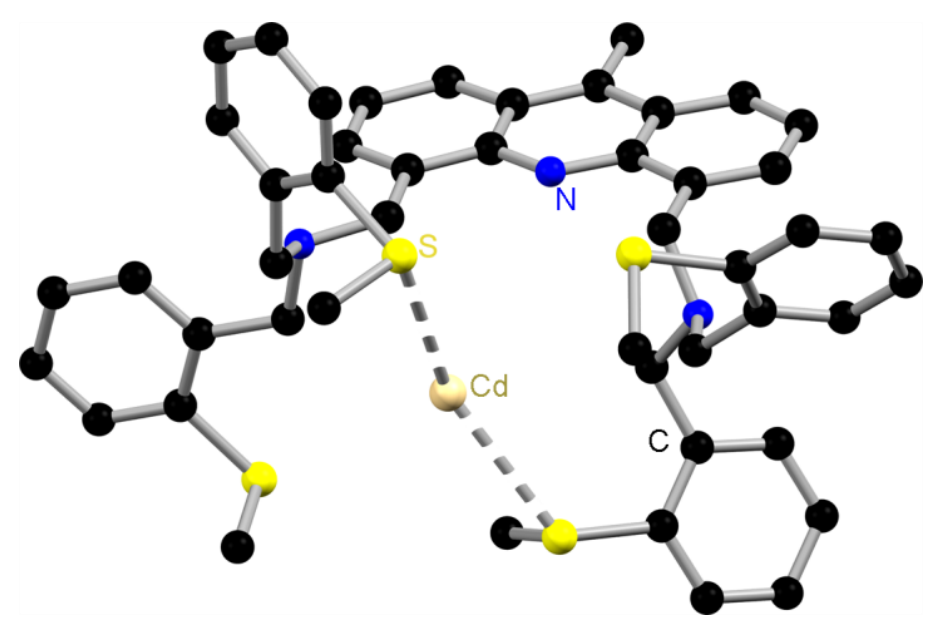

Figure 2.18: Possible interaction of 10 with a $\mathrm{Cd}^{2+}$ ion. The geometry was optimised with Avogadro v1.1.1 ${ }^{[96]}$ using the universal force field.

What more can be seen are the long $\mathrm{N}-\mathrm{Cd}$ distances which could explain the lack of changes in the spectra. Their lone pairs even point in the opposite direction which is due to the steric demand of the large receptor units and because of the repulsion of the multitude of hydrogen atoms. As mentioned before, without a coordination of the nitrogen lone pair to the analyte, the quenching mechanism of the PET effect is still present and no notable fluorescence emission can occur.

In conclusion, a new acridine derivative was synthesised and could be characterised completely by analytical methods like NMR spectroscopy and mass spectrometry. The X-ray diffraction of the single crystal provided structural information about the potential molecular sensor and, especially, the arrangement of the six possible donor atoms of the chelate. The compound was furthermore investigated by fluorescence spectroscopy and tested for metal ion recognition by the example of $\mathrm{Cd}^{2+}$ and $\mathrm{Hg}^{2+}$ ions. The envisaged PET effect by the use of the fluorophore-spacerreceptor system was proven and could be switched off by protonation of the amines. Further measurements with a variety of analytes would have been interesting. However, the synthesis of the amine itself and the subsequently synthesis of the target compound was declared as a too elaborate procedure considering the sparsely promising results.

\subsubsection{4,5-Bis $\left(N, N^{\prime}, N^{\prime}\right.$-trimethylethylenediaminemethylene)acridine (11) and its 9- methyl derivative (12)}

On the basis of the results of the investigation of $\mathbf{1 0}$, the amine $N, N^{\prime}, N^{\prime}$-trimethylethylenediamine (H-TrMEDA) was chosen for the substitution of the bromine atoms at the 4,5-positions. It has a less steric demand and its ethylene group offers more flexibility in comparison to the phenyl containing substituent. Another substantial factor is the selection of a receptor containing only nitrogen atoms. This improves the chance to coordinate a target ion by the PET initiating amine groups. Heteroatoms like sulfur have a different target affinity due to their large and high polarisable electron shell. This could lead to the possibility that they do not share a target ion with the smaller and harder nitrogen atoms. According to the receptor unit, various examples can be found in the literature, ${ }^{[31,98]}$ illustrating the good chelating effect of the amine. In Figure 2.19, a very promising compound is illustrated published by Czarnik et al. ${ }^{[97]}$ 

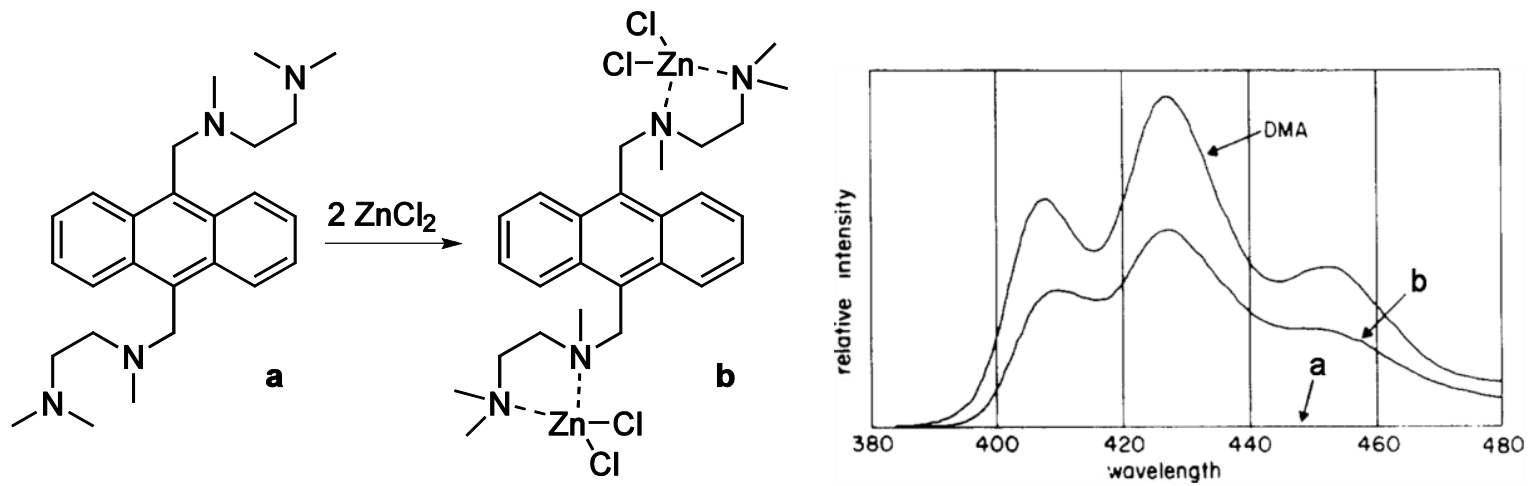

Figure 2.19: Left: Proposed coordination motif of bis(tmeda)anthracene with $\mathrm{ZnCl}_{2}$; Right: Fluorescence emission spectra of compounds $\mathbf{a}, \mathbf{b}$, and 9,10-dimethylanthracene (DMA) in acetonitrile $\left(10^{-4} \mathrm{M}\right) .{ }^{[97]}$

They are using the related fluorophore anthracene and the same quenching mechanism (PET). When the authors add $\mathrm{ZnCl}_{2}$ to a solution of 9,10-bis(tmeda)anthracene in acetonitrile, the emission intensity of the system is enhanced by a factor of 1000 . With acridine as fluorophore unit, only one similar structure could be found in the literature, published by Sapi et al. in 2009 (Scheme 2.15). ${ }^{[99]}$ The aim of the authors was the evaluation of the derivatives as telomeric Gquadruplex binding agents. Therefore, no information was available about their sensing properties for e.g. metal ions.<smiles>[R]Cc1cccc2cc3cccc(C[R])c3nc12</smiles>

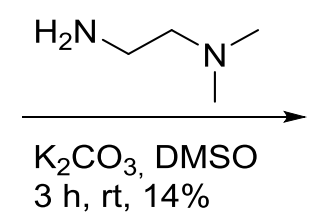<smiles>CN(C)CCNCc1cccc2cc3cccc(CNCCN(C)C)c3nc12</smiles>

Scheme 2.15: Synthesis of 4,5 -bis $\left(N^{\prime}, N^{\prime}\right.$-dimethylethylenediaminemethylene $)$ acridine $(\mathrm{R}=\mathrm{Cl}) .{ }^{[99]} 2.2$ equivalents of the primary amine were used.

They synthesised several 4,5-bis(dialkylaminoalkyl)-substituted acridines starting from the hydroxymethyl $(\mathrm{R}=\mathrm{OH})$ or halogenomethyl derivative $(\mathrm{R}=\mathrm{Cl}, \mathrm{Br})$. In this thesis, the reaction pathway was modified at various points. As already used before, the bromomethylene derivative as starting material should lead to higher yields because of the better leaving group ( $\mathrm{Br}$ vs $\mathrm{Cl}$ ). The solvent DMSO is exchanged by acetonitrile which allows a direct work-up with brine to remove the excess of salt. Secondly, it can be more easily removed under reduced pressure. However, the main important part is the choice of a primary or secondary amine. Primary amines tend to undergo oligomeric reactions, ${ }^{[42]}$ which is why the amine H-TrMEDA was chosen for this synthesis.<smiles>[R]c1c2cccc(CBr)c2nc2c(CBr)cccc12</smiles><smiles>[R]c1c2ccccc2c(CN(C)CCN(C)C)c2nc3c(CN(C)C)cccc3c([R])c12</smiles>

11: $\mathrm{R}=\mathrm{H}$

12: $R=M e$

Scheme 2.16: Reaction pathway to the TrMEDA derivatives $11(R=H)$ and $12(R=M e)$. 
Another positive benefit is the $+\mathrm{I}$ effect of the methyl groups. They push electron density into the nitrogen atoms and, therefore, enhance the donor ability of their lone pair. This is crucial for an efficient receptor unit. Both derivatives $\mathbf{8}$ and $\mathbf{9}$ were used as starting materials, whereas $\mathbf{8}$ has already been used for the synthesis of $\mathbf{1 1}$ in the master thesis. ${ }^{[63]}$ In this thesis, the synthesis has to be optimised and the fluorescence measurements have to be repeated because the first where so low that the material could not be obtained in useful quantities and in the letter there were some systematic errors identified. The synthesis was performed several times with slight changes in the reaction temperature, time and used solvent. It turned out that the reaction conditions in Scheme 2.16 were the best. Reaction temperatures below $50{ }^{\circ} \mathrm{C}$ did not lead to the desired product over a couple of days. Unfortunately, the yield of $\mathbf{1 1}$ could only be improved to 14\%. After the extraction of the product with ethyl acetate in a brine solution, the ${ }^{1} \mathrm{H}$ NMR spectrum showed some small but unwanted signals left. Since they were especially in the aromatic region, this sideproduct could affect the fluorescence studies. However, every attempt to remove this compound was unsuccessful. The column chromatography could in some cases reduce the amount of the side-product but always along with a strong reduction of the yield. Other attempts like the quaternisation of the product or the precipitation with a zinc salt were not satisfying. Since $\mathbf{1 1}$ is an oily product, a (re-)crystallisation could not be implemented.

The synthesis of the methyl derivative $\mathbf{1 2}$ was carried out in $\mathrm{MeCN}$ at $50{ }^{\circ} \mathrm{C}$ with an excess of $\mathrm{K}_{2} \mathrm{CO}_{3}$ and 2.1 equivalents of the amine. The reaction mixture was stirred for 16 hours and afterwards purified by column chromatography on alumina. This procedure gave a yield of $17 \%$ which was the highest value along with a pure product.

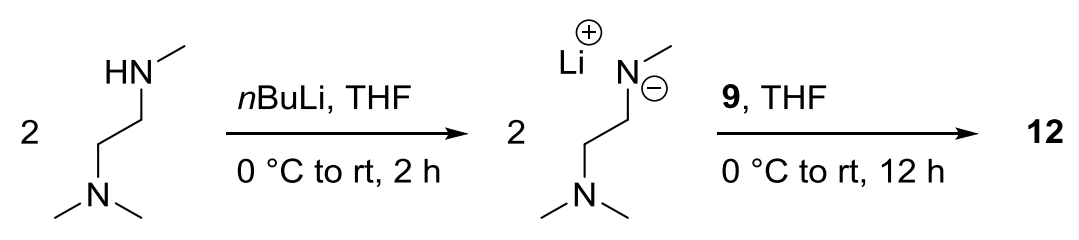

Scheme 2.17: Second approach to 12 with $n$ BuLi and $N, N^{\prime}, N^{\prime}$-trimethylethylenediamine (H-TrMEDA).

Since the yields of the desired products were not very pleasing, a second approach for the synthesis of $\mathbf{1 2}$ was tried (Scheme 2.17). Therefore, the used amine was deprotonated before with the strong base $n$-butyl lithium and was afterwards added to the dissolved acridine derivative 9 .

This attempt yielded a variety of side-products which reduced the solubility in the commonly used NMR solvents $\mathrm{CDCl}_{3}$ and DMSO- $d_{6}$. However, the ${ }^{1} \mathrm{H}$ NMR spectrum of the reaction mixture showed an almost complete conversion of the starting material and most likely signals of the desired product (Figure 2.20, b). As can be seen from the comparison of the spectra (b) and (c), the quantity of non-defined signals using $n \mathrm{BuLi}$ is higher than with the common used and milder base $\mathrm{K}_{2} \mathrm{CO}_{3}$. This is why the second approach to $\mathbf{1 2}$ was no promising alternative. 


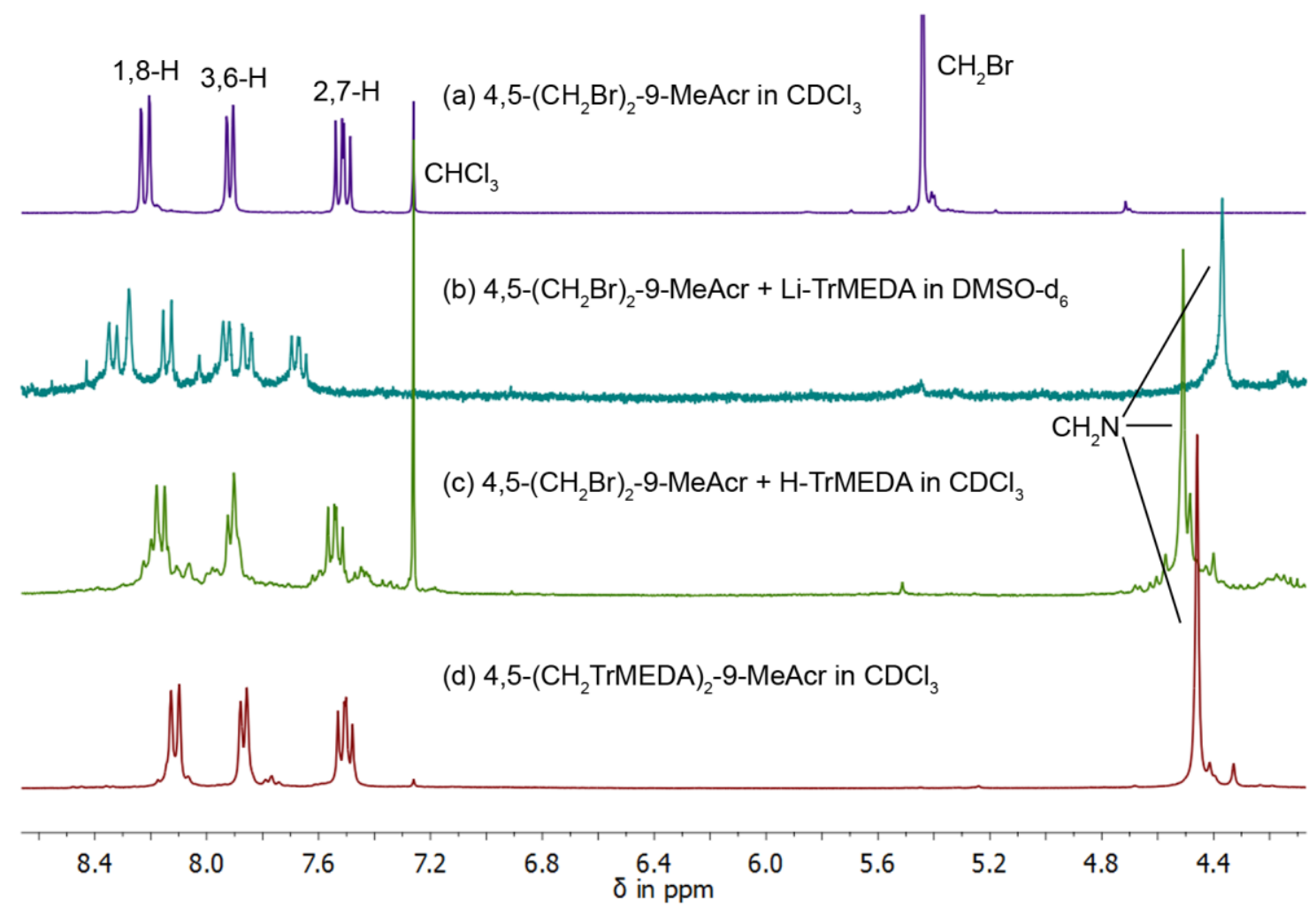

Figure 2.20: Illustration of the ${ }^{1} \mathrm{H}$ NMR spectra of the starting material 9 (a) and the purified product 12 (d). The spectra in-between compare the conversion of 9 with the lithiated amine (b) and with $\mathrm{K}_{2} \mathrm{CO}_{3}$ and H-TrMEDA (c); both are measured out of the reaction mixture before purification.

\subsubsection{Luminescence properties of 4,5-bis $\left(N, N^{\prime}, N^{\prime}\right.$-trimethylethylenediamine- methylene)acridine (11)}

As mentioned before, $\mathbf{1 1}$ has already been synthesised during the master thesis. ${ }^{[63]}$ However, the fluorescence measurements were performed with an excess of $n \mathrm{Bu}_{4} \mathrm{NBF}_{4}$ at that time, to keep the ionic strength constant. Later investigations showed that this salt contained impurities which led to an unintended protonation of the ligand and distorted results.
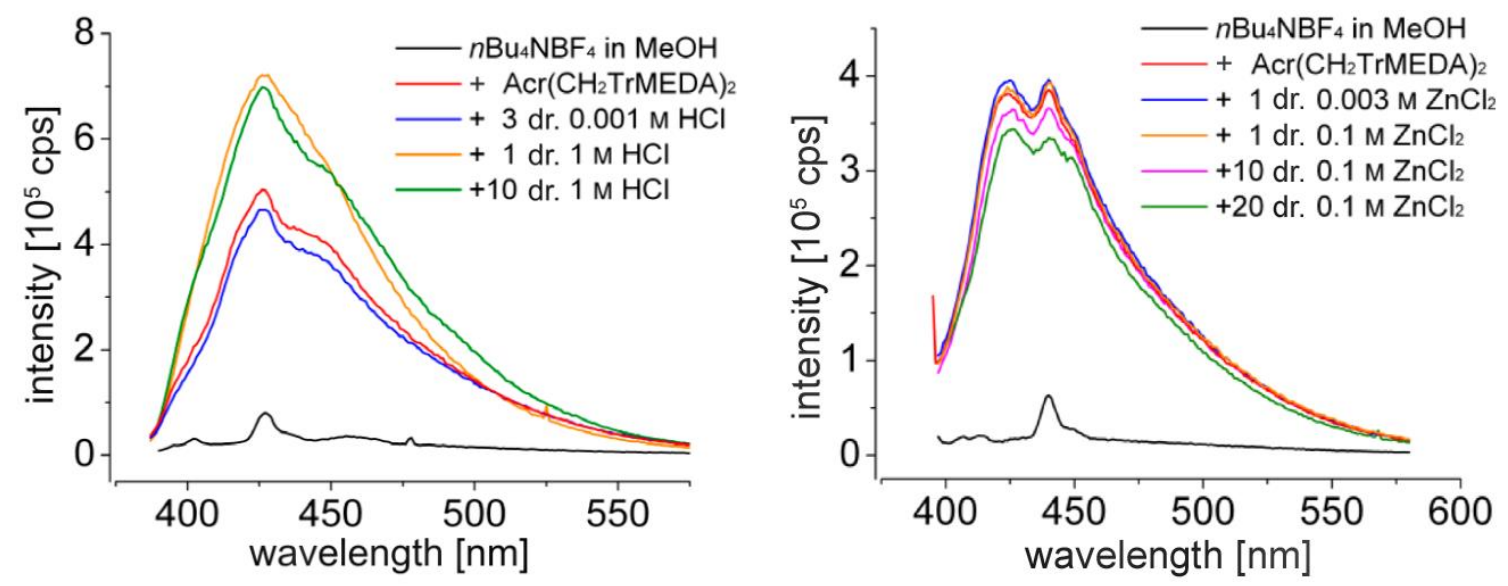

Figure 2.21: Former investigations to 11 in $\mathrm{MeOH}\left(\mathrm{c}=1 \cdot 10^{-4} \mathrm{M}, \lambda_{\mathrm{exc}}=380 \mathrm{~nm}\right)$ with an excess of $n \mathrm{Bu}_{4} \mathrm{NBF}_{4}{ }^{[63]}$ Left: addition of aqueous $\mathrm{HCl}$; right: addition of $\mathrm{ZnCl}_{2}$. 
Actually, the ligand $\mathbf{1 1}$ should show similar fluorescence properties like $\mathbf{1 0}$ as its structure has the same "fluorophore-spacer-receptor" design. Therefore, only less emission intensity should be expected for a $10^{-4} \mathrm{M}$ solution in methanol. The starting intensity of $5 \cdot 10^{5} \mathrm{cps}$ (red line) is, however, very high and is only slightly enhanced with an excess of aqueous hydrochloric acid (Figure 2.21, left). Consequently, a screening procedure with a variety of metal salts did not alter the spectra significantly, exemplarily illustrated by a titrating experiment with $\mathrm{ZnCl}_{2}$ (Figure 2.21, right).

In this thesis, it was of great interest whether $\mathbf{1 1}$ would react to different analytes when the right conditions are applied. The $n \mathrm{Bu}_{4} \mathrm{NBF}_{4}$ salt has been left out with regard to the noted problem and the concentration was lowered to a $10^{-5} \mathrm{M}$ solution in methanol. The resulting emission spectrum (Figure 2.22, left) differs significantly by its intensity $\left(<1 \cdot 10^{4} \mathrm{cps}\right)$ and wavelength in comparison to the spectrum depicted above. Without the protonation of the amines, the PET effect seems to work as expected and quenches the fluorescence emission. The highest peaks at $405 \mathrm{~nm}$ for the excitation spectrum and $421 \mathrm{~nm}$ for the emission spectrum are a result of Raman scattering of the used solvent. ${ }^{[100]}$ The broad band of the emission spectrum has its maximum at about $462 \mathrm{~nm}$ which is less red-shifted as compound $\mathbf{1 0}(505 \mathrm{~nm})$.
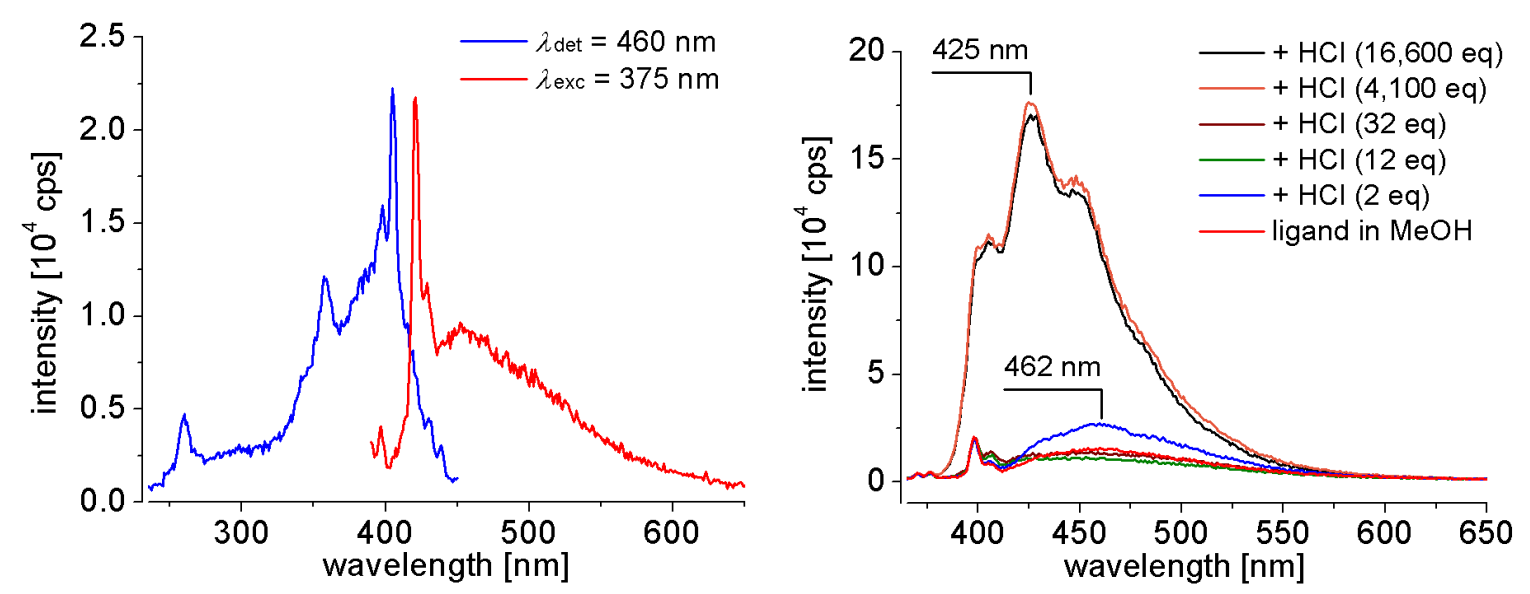

Figure 2.22: Left: Excitation (blue: $\lambda_{\mathrm{det}}=460 \mathrm{~nm}$ ) and emission (red: $\lambda_{\mathrm{exc}}=375 \mathrm{~nm}$ ) spectrum of $11 \mathrm{in}$ $\mathrm{MeOH}\left(10^{-5} \mathrm{M}\right)$; right: emission spectra of $\mathbf{1 1} \mathrm{in} \mathrm{MeOH}$ with addition of aqueous $\mathrm{HCl}\left(\lambda_{\mathrm{exc}}=357 \mathrm{~nm}\right)$.

The addition of small amounts of aqueous hydrochloric acid (2 to $32 \mathrm{eq}$ ) did not lead to a significant change of the emission shape or intensity (Figure 2.22, right). An excess of $\mathrm{HCl}$, however, resulted in a huge altering of the spectrum. The emission wavelength is blue-shifted from 462 to $425 \mathrm{~nm}$ and the intensity rises from $1.0 \cdot 10^{4} \mathrm{cps}$ to $17.5 \cdot 10^{4} \mathrm{cps}$. In contrast to $\mathbf{1 0}$, the structure of the excitation spectrum of $\mathbf{1 1}$ was not significantly altered by the addition of $\mathrm{HCl}$. Therefore, the change in emission intensity can directly be compared. Additionally, the broad band becomes more structured with three well-defined maxima. The protonation of the ligand has likely led to larger energy distances between the vibrational states which can be resolved now.

With a view at the strong shift of the wavelength, it is worth thinking about the possibility that also the nitrogen atom of the acridine backbone gets protonated by the addition of larger amounts of acid. The $\mathrm{p} K_{\mathrm{a}}$ values of the amine groups lie around 9 for the outer nitrogen ${ }^{[101]}$ and around 10 for the inner nitrogen atom ${ }^{[102]}$. Acridine itself has a $\mathrm{p} K_{\mathrm{a}}$ value of 5.45 , but it rises to 10.7 when getting excited in the fluorescence spectrometer. ${ }^{[103]}$ Consequently, the fluorophore in the excited 
state should get protonated first, but with a slight excess of acid the amine groups take up a proton, too. In contrast to this consideration is a publication of Tripathi et al. ${ }^{[104]}$ They investigated the fluorescence properties of 9-aminoacridine at various $\mathrm{pH}$ values (Figure 2.23).

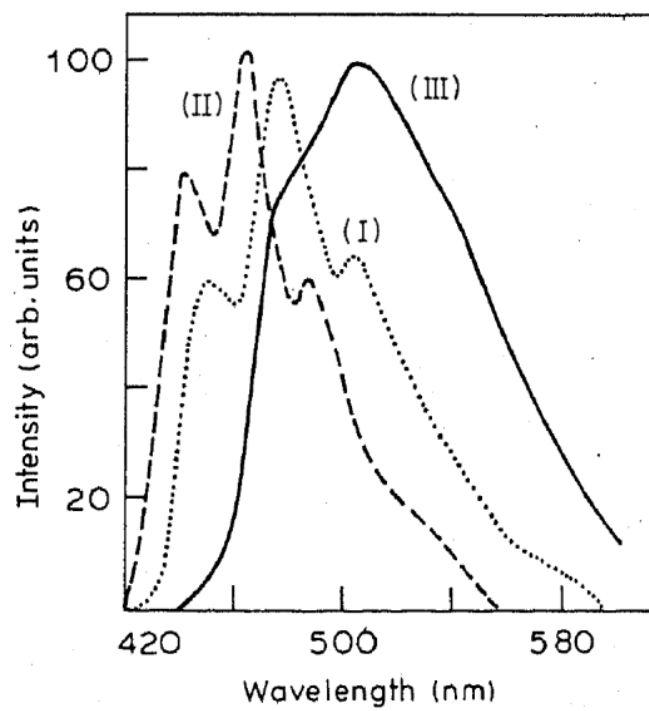<smiles>Nc1c2ccccc2nc2ccccc12</smiles>

(I)<smiles>[NH3+]c1c2ccccc2nc2ccccc12</smiles>

(II)<smiles>[NH3+]c1c2ccccc2[nH+]c2ccccc12</smiles>

(III)

Figure 2.23: Emission spectra of 9 -aminoacridinium hydrochloride at different $\mathrm{pH}$ values ${ }^{[104]}: \mathrm{pH}=12$ (I), $\mathrm{pH}=6$ (II), and in conc. $\mathrm{H}_{2} \mathrm{SO}_{4}$ (III).

They have shown that the emission spectrum of 9-aminoacridine in water is slightly blue-shifted when it gets protonated at the primary amine $(\mathrm{pH}=6)$. Only when the compound is measured in concentrated $\mathrm{H}_{2} \mathrm{SO}_{4}$, the emission is strongly red-shifted with some loss of structure. The formed emission curve is similar to that of acridinium ion which often can be found in literature ${ }^{[105]}$ as well as in the investigations of the master thesis. ${ }^{[63]}$ The same observations like the blue shift and the structured emission curve was made during the $\mathrm{HCl}$ experiment of $\mathbf{1 1}$ (Figure 2.22, right). It is, therefore, very likely that either the $\mathrm{p} K_{\mathrm{a}}$ of the excited acridine backbone is not reaching this high value of 10.7 if it is substituted with the receptor units; or the wavelength-dependent proton transfer to the aromatic system is not favoured at the used excitation wavelength. ${ }^{[105 a]}$ These considerations will be supported by later investigations in this thesis.

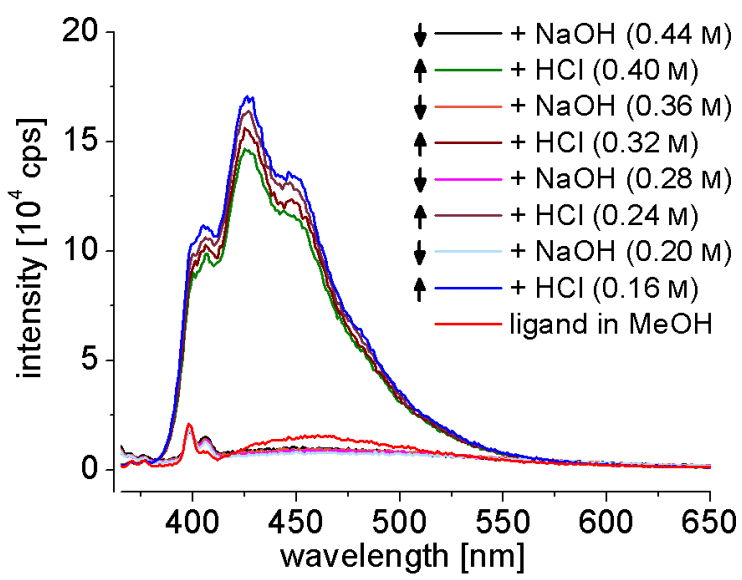

Figure 2.24: Reversibility study with alternating addition of aqueous $\mathrm{HCl}$ and $\mathrm{NaOH}$ to a solution of $\mathbf{1 1}$ in methanol $\left(10^{-5} \mathrm{M}, \lambda_{\text {exc }}=357 \mathrm{~nm}\right)$.

A further important analysis upon the addition of a strong acid is the question if the compound gets protonated as expected or if a decomposition product generates the altered spectrum. 
Therefore, a reversibility experiment of the protonation/deprotonation process of the ligand was implemented. A specified amount of aqueous $\mathrm{HCl}$ and $\mathrm{NaOH}$ was alternately added to the compound solution (Figure 2.24). The experiment demonstrates the good stability of the ligand in a low concentrated alcoholic solution. The fluorescence emission rises strongly with the addition of $\mathrm{HCl}$ and is completely quenched after the addition of an appropriate amount of $\mathrm{NaOH}$. The maximum emission intensity decreases only slightly after each cycle which is not necessarily due to decomposition processes. The amount of acid and base was given dropwise with a glass pipette which could have small variations in volume. Moreover, the added $\mathrm{Cl}^{-}$ions are known to act as fluorescence quencher. ${ }^{[106]}$

These results are nice features for a molecular sensor. It reacts to an analyte $\left(\mathrm{H}^{+}\right.$ions) with a strong increase in fluorescence intensity as well with a significant shift of the emission wavelength. The process is reversible along with a stable sensor ligand. Accordingly, a metal ion screening (Chapter 2.2.6) was carried out to check if $\mathbf{1 1}$ would react equally to some of the tested cations. The screening was performed in methanol $\left(10^{-5} \mathrm{M}\right)$ with an excitation wavelength of $357 \mathrm{~nm}$ and is summarised in the following bar chart (Figure 2.25).

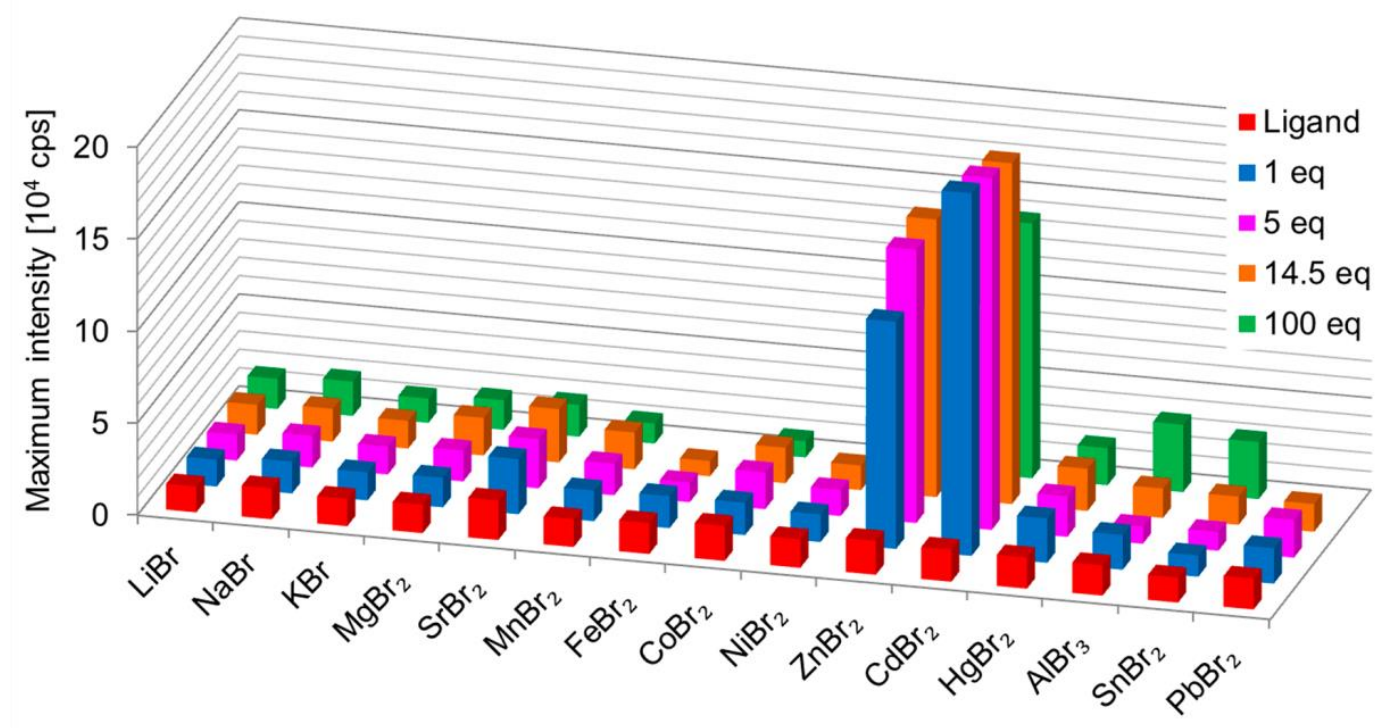

Figure 2.25: Cation screening of $\mathbf{1 1}$ in methanol $\left(10^{-5} \mathrm{M}, \lambda_{\mathrm{exc}}=357 \mathrm{~nm}\right)$ with different metal salts from 1 to 100 equivalents. The maximum emission intensity is depicted for each metal ion addition.

For every addition, the maximum emission intensity is illustrated and can directly be compared among each other. In the case of $\mathrm{FeBr}_{2}$, the solution got coloured at higher concentrations which only resulted in the absorption of the beam. $\mathrm{NiBr}_{2}$ and $\mathrm{PbBr}_{2}$ were not soluble enough for measurements with 100 eq of the salts. What is conspicuous is the high selectivity of the ligand towards $\mathrm{Zn}^{2+}$ and $\mathrm{Cd}^{2+}$ ions among the tested metal salts. The intensity rises strongly and the wavelength of the maximum is shifted from $462 \mathrm{~nm}$ to 456 or $450 \mathrm{~nm}$, respectively, in the case of a $\mathrm{Zn}^{2+}$ or $\mathrm{Cd}^{2+}$ coordination (Figure 2.26). The difference of $6 \mathrm{~nm}$ between the two metal ion titrations is quite small and can just be differentiated by fluorescence spectroscopy. The emission intensity is a bit more increased by the addition of cadmium ions to the ligand solution. However, the maximum intensity is already reached with the addition of one or five equivalents in both cases. The factor of the enhancement lies around 10 ( 8 for $\mathrm{Zn}^{2+}$ and 11 for $\mathrm{Cd}^{2+}$ ions) which is the 
same as for the protonation experiment. With 100 eq added, the intensity decreases mainly due to dilution effects ( $0.9 \mathrm{ml}$ of $\mathrm{ZnBr}_{2}$ onto $3.0 \mathrm{ml}$ solution in the cuvette).
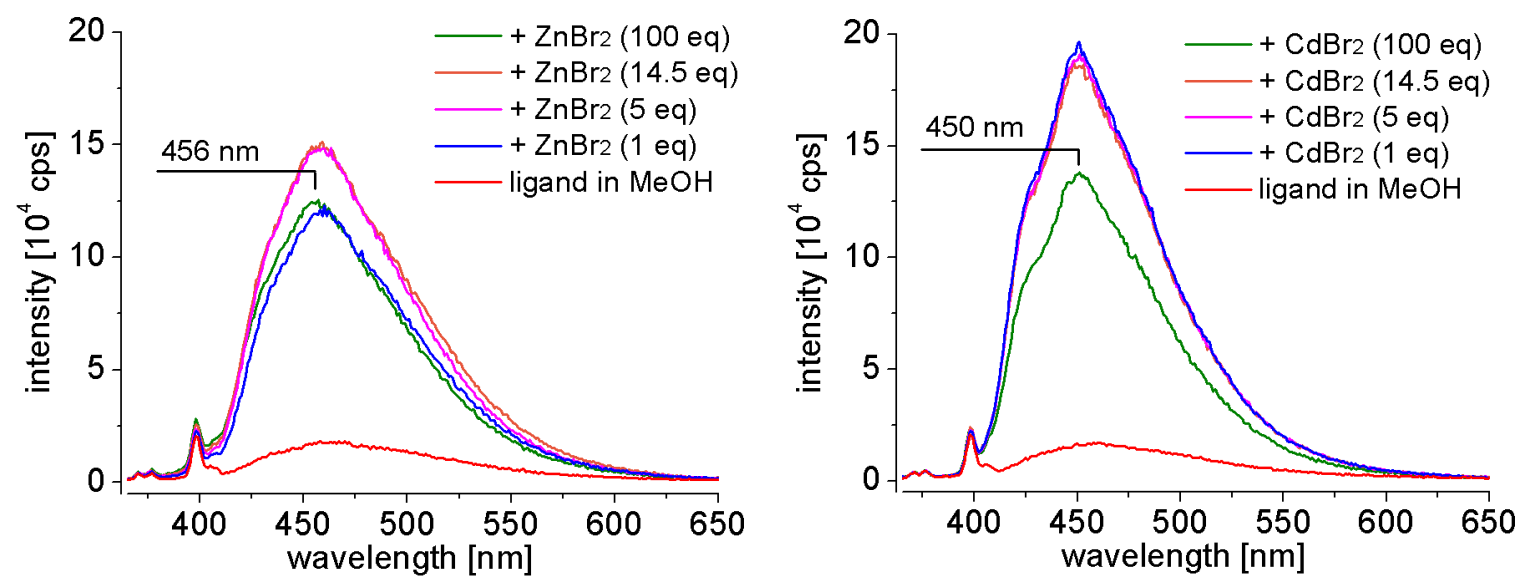

Figure 2.26: Emission spectra of 11 in methanol $\left(10^{-5} \mathrm{M}, \lambda_{\mathrm{exc}}=357 \mathrm{~nm}\right)$ with addition of $\mathrm{ZnBr}_{2}$ (left) and $\mathrm{CdBr}_{2}$ (right). The emission wavelength of the pure ligand (red line) is around $462 \mathrm{~nm}$.

In the case of zinc, a second salt was dissolved and titrated to the ligand to check how the anion influences the emission spectrum. In contrast to the used halide salts a chelating anion was chosen: $\mathrm{Zn}\left(\mathrm{NO}_{3}\right)_{2} \cdot 6 \mathrm{H}_{2} \mathrm{O}$. Furthermore, the higher Lewis basic character of the nitrate oxygen atoms may reduce the positive charge of the zinc ion which makes it less attractive for the lone pairs of the nitrogen atoms of 11. Nevertheless, an even higher fluorescence enhancement in comparison to $\mathrm{ZnBr}_{2}$ was observed after the addition of $\mathrm{Zn}\left(\mathrm{NO}_{3}\right)_{2}$ (Figure 2.27).

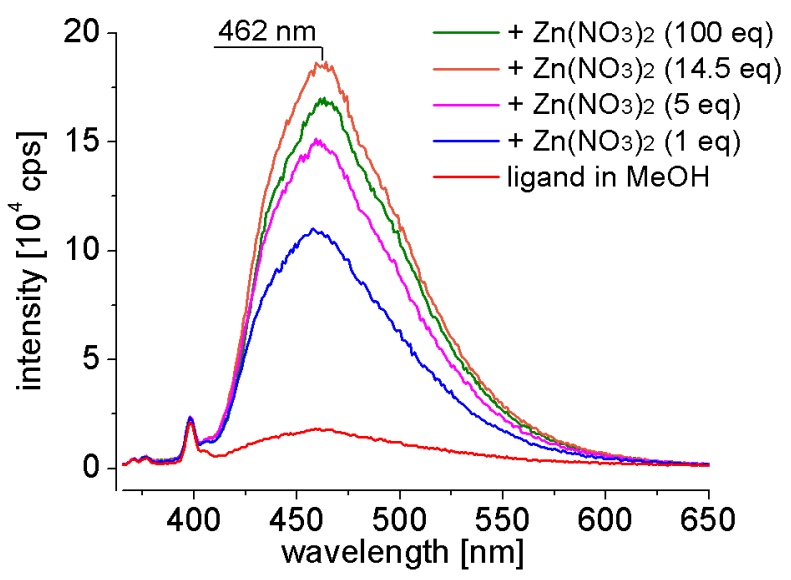

Figure 2.27: Emission spectra of 11 in methanol $\left(10^{-5} \mathrm{M}, \lambda_{\mathrm{exc}}=357 \mathrm{~nm}\right)$ with addition of $\mathrm{Zn}\left(\mathrm{NO}_{3}\right)_{2} \cdot 6 \mathrm{H}_{2} \mathrm{O}$.

The emission intensity rises strongly and reaches a value which lies somewhere between the results of the zinc and cadmium bromide titrations. More interestingly, the wavelength of the formed complex has the same value than the pure ligand $(462 \mathrm{~nm})$. This is in contrary to the bromide experiments where the wavelengths shift to 456 and $450 \mathrm{~nm}$ for zinc and cadmium, respectively. As mentioned before, the zinc ion is maybe reduced in its positive charge by the counter ions and, therefore, does not get this close to the acridine ring. This lowers its influence to the $\pi$ system which is reflected in the emission wavelength. The differentiation between zinc and cadmium ions with this sensor system is hardly possible if nothing is known about the anions in solution. More investigations with different anions were needed for more accurate statements. 

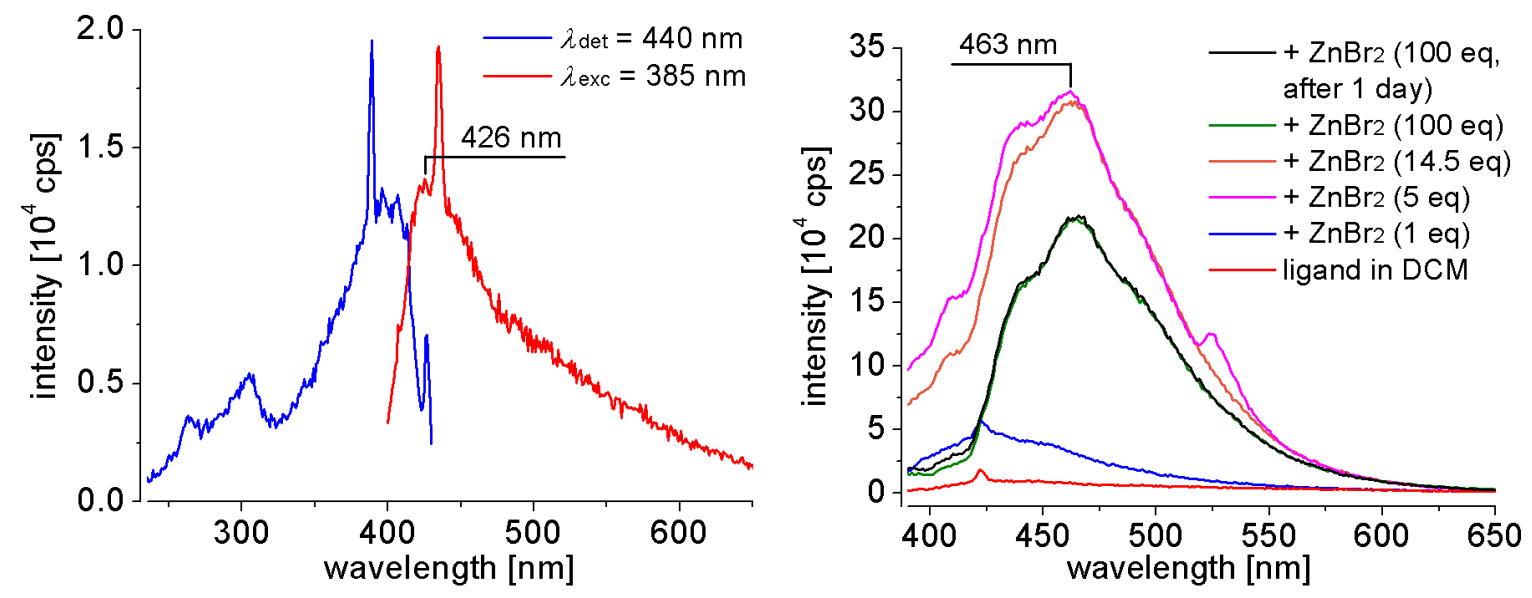

Figure 2.28: Left: Excitation (blue: $\lambda_{\mathrm{det}}=440 \mathrm{~nm}$ ) and emission (red: $\lambda_{\mathrm{exc}}=385 \mathrm{~nm}$ ) spectrum of 11 in $\operatorname{DCM}\left(10^{-5} \mathrm{M}\right)$; right: emission spectra of 11 in DCM with addition of $\mathrm{ZnBr}_{2}\left(\lambda_{\mathrm{exc}}=375 \mathrm{~nm}\right)$.

In addition, it was interesting to know if the ligand behaves different in a less polar and aprotic solvent. That is why dichloromethane was chosen for a comparison experiment. The metal salts of the screening procedure are non-soluble in $\mathrm{DCM}$ except $\mathrm{ZnBr}_{2}$. So, an excitation and emission spectrum of 11 was recorded, which was afterwards titrated with $\mathrm{ZnBr}_{2}$ (Figure 2.28). The emission spectrum of $\mathbf{1 1}$ is shifted hypsochromic $(426 \mathrm{~nm})$ in comparison to the measurement in methanol (462 nm, Figure 2.22). This is a known effect caused by the difference in polarity of the used solvents. ${ }^{[107]}$ The fluorophore usually has a larger dipole moment in the excited state $\left(\mu_{\mathrm{E}}\right)$ than in the ground state. Polar solvents are able to reorient around $\mu_{\mathrm{E}}$ and lower the energy of the excited state. This results in an emission at higher wavelengths. Less polar solvents have only a small effect to $\mu_{\mathrm{E}}$ and do not shift the emission maximum strongly. This behaviour can only be observed with polar fluorophores. An unsubstituted anthracene would not show any solvent dependency. The theory to this effect as well as an illustrative experiment with 4-dimethylamino4'-nitrostilbene (DNS) is depicted in Figure 2.29.
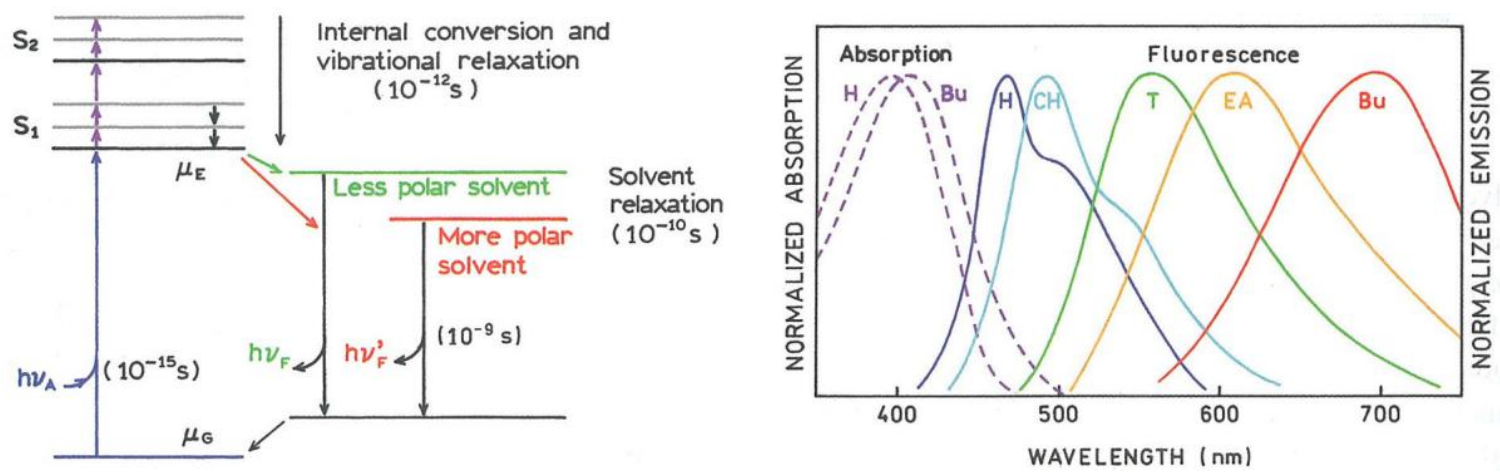

Figure 2.29: Left: Jablonski diagram for the illustration of the solvent relaxation effect depending on the solvent polarity. Singlet state $(\mathrm{S})$, dipole moment of the excited state $\left(\mu_{\mathrm{E}}\right)$ and of the ground state $\left(\mu_{\mathrm{G}}\right)$, absorption $\left(\mathrm{h} v_{\mathrm{A}}\right)$, fluorescence $\left(\mathrm{h} v_{\mathrm{F}}\right)$; right: Emission spectra of DNS in solvents of increasing polarity. Hexane $(\mathrm{H})$, cyclohexane $(\mathrm{CH})$, toluene $(\mathrm{T})$, ethyl acetate $(\mathrm{EA}), n$-butanol $(\mathrm{Bu}) .{ }^{[107]}$

As already described before, the sharp and highest peaks in Figure 2.28, left, are caused by Raman scattering of the used solvent (389 and $435 \mathrm{~nm}) .{ }^{[108]}$ The intensity of the excitation and emission spectrum is quite low, but with the addition of $\mathrm{ZnBr}_{2}$, the emission rises tremendously. 
The increase is approximately four times stronger than with the solvent methanol (a factor of 32 in comparison to 8). However, with only one equivalent of zinc ions added, the effect is less pronounced. Likewise to the addition of $\mathrm{CdBr}_{2}$, the emission intensity decreases when too high concentrations of the salt are reached. Additionally, a bathochromic shift of the emission wavelength along with an altering of the curve shape is observable. The maximum is shifted by approximately $37 \mathrm{~nm}$. This is a significant value which is as high as at the addition of hydrochloric acid to the ligand solution. The shape of the emission curve after the zinc addition has one maximum with two shoulders on both sides. In general, it becomes more structured. Furthermore, the stability of the complex in solution was tested in this experiment. The cuvette was sealed with a lid for 24 hours whereupon a further emission spectrum was recorded. No alteration of the spectrum was noticed after this period of time.

Since the shape and the wavelength of the emission curve changes during the titration, the excitation spectrum needs to be observed, too. In Figure 2.30, the excitation spectra of the pure ligand and with an amount of $\mathrm{ZnBr}_{2}$ are depicted, as well in DCM as in methanol. The long black line perpendicular to the spectra shows the wavelength at which the solution was excited.
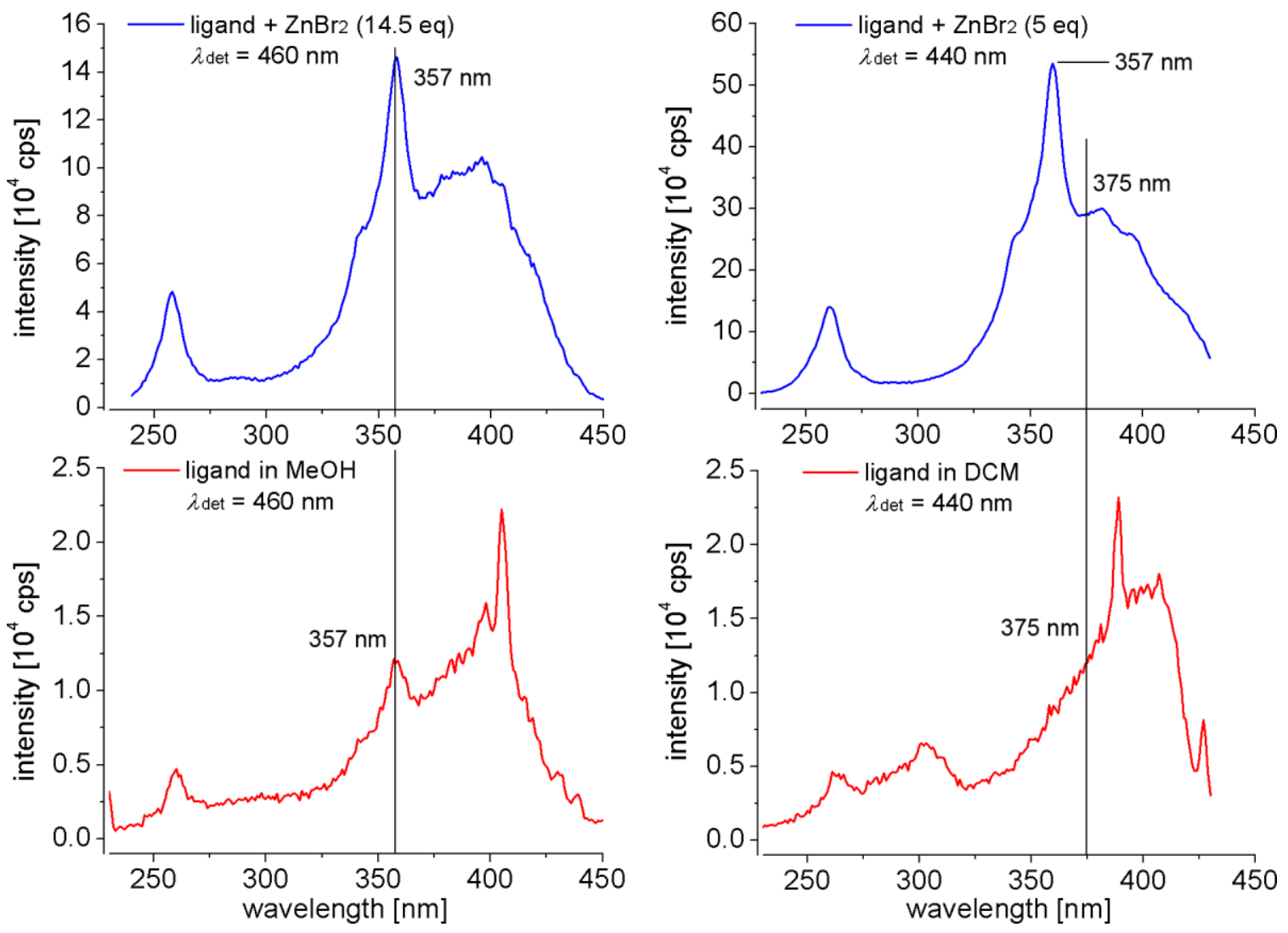

Figure 2.30: Comparison of the excitation spectra of 11 without (bottom) and with addition of $\mathrm{ZnBr}_{2}$ (top); (left: in $\mathrm{MeOH}$ with $\lambda_{\mathrm{det}}=460 \mathrm{~nm}$; right: in DCM with $\lambda_{\text {det }}=440 \mathrm{~nm}$ ).

In the case of methanol measured before, the spectrum looks quite the same before and after the addition of zinc ions (left picture). Only the intensity output is higher for the latter which is why the Raman signal above $400 \mathrm{~nm}$ vanishes. Looking at the spectra in DCM, a complete new band is rising at $357 \mathrm{~nm}$, the same value as in methanol. If the DCM sample was excited at $357 \mathrm{~nm}$, the emission intensity would have been even higher than it is already. 
When comparing the shifts of the emission maxima between the methanol and the dichloromethane experiment, it is apparent that the zinc ions in DCM have a greater effect to the fluorescence system ( $\Delta \lambda 6 \mathrm{~nm}$ vs $37 \mathrm{~nm}$ ). This observation is maybe due to the weaker donor ability of the chlorinated solvent in comparison to the polar alcohol. As a consequence, the zinc ions have a higher positive charge and bind stronger to the amines of the receptor ligand. This should at first suppress the PET effect resulting in stronger emission intensity, which is the case here. Secondly, the shorter $\mathrm{N}-\mathrm{Zn}$ distances may have an electronic influence to the $\pi$-system of the fluorophore. Accordingly, varying the solvents with different dielectric constants can have significant influences to the fluorescence spectra. The starting emission wavelength differs if the sensor ligand is polar, too, which can be an important property for some experimental demands. The influence of the solvent shell to the analyte should never be neglected. This can have an effect to different sensory aspects as already discussed before. However, concerning the worse solubility of a variety of metal salts in less polar solvents than methanol, it is more useful to stick with one polar solvent. The comparability of the experiments and the similarity to water are crucial reasons for that.

A general disadvantage of the compound is its non-solid state of aggregation. The oily product hampers the usual work-up procedures as well as the crystallisation of the compound. The latter is a serious disadvantage since the solid state structures of the free ligand and especially of its metal complexes provides a lot of information. The manner of the analyte complexation is needed to understand the fluorescence behaviour described in this thesis. As already mentioned before in Chapter 2.2.7, computational studies can give a hint how a possible interaction of the ligand with a metal ion might look like. The organic ligand was geometry optimised by Avogadro ${ }^{[96]}$ and again optimised after the addition of a zinc ion close to the receptor units. In this case, two structures with different coordination spheres were obtained depending on the initial position of the metal ion. The resulting atom coordinates were imported to the $\mathrm{ORCA}^{[109]}$ program package to determine the minimum energies of both complexes. The optimised structures are illustrated in Figure 2.31.
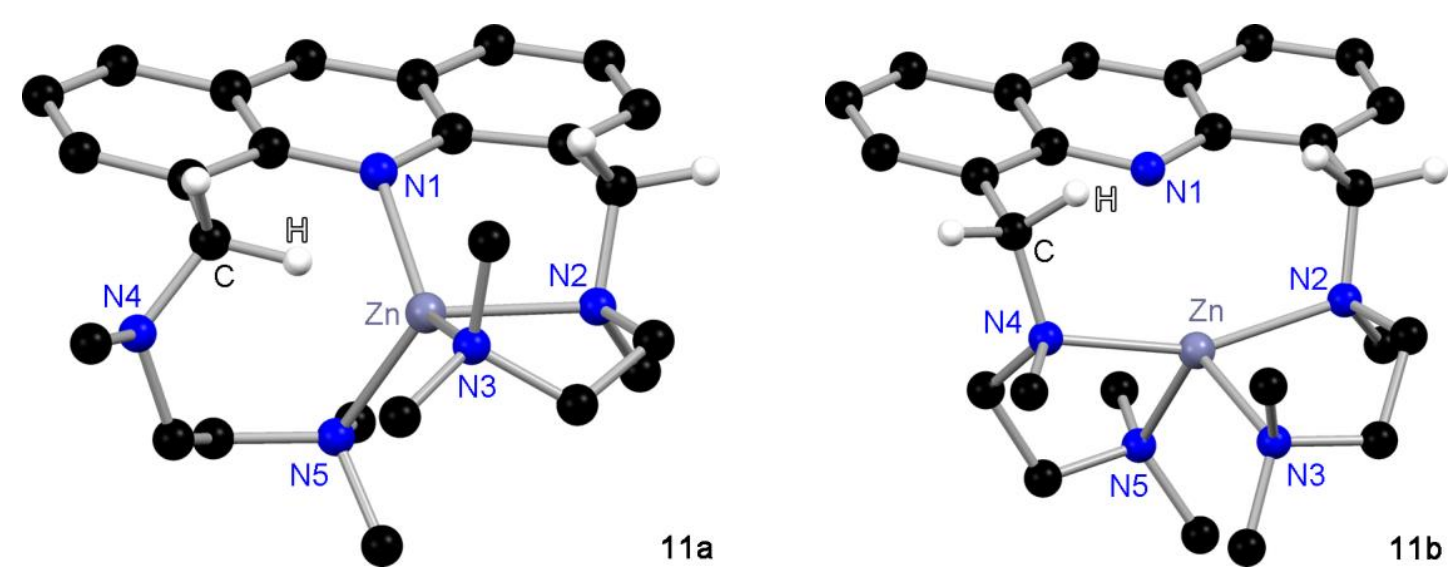

Figure 2.31: Possible interactions of 11 with a $\mathrm{Zn}^{2+}$ ion. The geometries were optimised with $\mathrm{ORCA}^{\text {[96] }}$ including all hydrogen atoms. In these illustrations, only the hydrogen atoms at the methylene units are shown.

During the geometry optimisation, the zinc ion gets chelated by the amine receptors. In the case of 11a, N4 is not involved in the complexation. However, this interaction is crucial for the fluorescence properties of the ligand system since N2 and N4 are relevant for the electron 
transfer. On the on hand, the emission intensity of the sensor system would only be 'switched on' half because the PET effect would still be present at this receptor site. On the other hand, the acridine nitrogen atom is strongly involved in the coordination of the cation, which is demonstrated by the calculated distances listed in Table 2.6. All N-Zn distances have similar values and are shorter than the distances of $\mathbf{1 1 b}$. Here, the acridine shows no or only very weak interaction, with a N1-Zn distance of $273.7 \mathrm{pm}(210.9 \mathrm{pm}$ in the case of 11a). The calculated distances of both structures represent the gas phase and are only comparable to each other. To determine which coordination motif is more likely, the Gibbs free enthalpies were computed. 11b has a smaller minimum energy indicating a more stable geometry. The complexes have an energy difference of $52.1 \mathrm{~kJ} \cdot \mathrm{mol}^{-1}$, which additionally demonstrates the main formation of $\mathbf{1 1 b}$.

Table 2.6: Selected atom distances $[\mathrm{pm}]$ of the calculated structures.

\begin{tabular}{ccc}
\hline & 11a & 11b \\
\hline \hline N1-Zn & 210.9 & 273.7 \\
N2-Zn & 210.9 & 212.9 \\
N3-Zn & 216.9 & 226.5 \\
N4-Zn & --- & 214.8 \\
N5-Zn & 210.2 & 212.3 \\
H-Zn & 203.2 & --- \\
\hline
\end{tabular}

To conclude, 4,5-bis $\left(N, N^{\prime}, N^{\prime}\right.$-trimethylethylenediaminemethylene)acridine has shown very interesting sensory abilities. It is able to detect small amounts of zinc or cadmium ions in a polar alcoholic medium. The present PET effect is working well with an easily visible 'off/on' principle. In contrast to some anthracene based chemical sensors investigated 2014 in this working group ${ }^{[25 \mathrm{e}]} \mathbf{1 1}$ is stable in a $10^{-5} \mathrm{M}$ solution for at least 24 hours even with the addition of $\mathrm{ZnBr}_{2}$ (Figure 2.28, right, black line). The alternating addition of an excess of aqueous $\mathrm{HCl}$ and $\mathrm{NaOH}$ also did not seem to decompose the ligand. Despite all these positive properties of 11, its synthesis involves several side-products resulting in a very low yield. The calculations with ORCA hinted to a coordination of a $\mathrm{Zn}^{2+}$ ion by the amine receptors not involving the acridine nitrogen atom. But without any experimental data of solid state structures, it is hard to make a statement. Nevertheless, two acridine derivatives were synthesised and characterised by NMR spectroscopy. 12 was synthesised for the first time, whereas 11 was already isolated during the master thesis. The latter could now be characterised in detail by fluorescence spectroscopy. It was waived to investigate the fluorescence properties of $\mathbf{1 2}$ since the methyl group at the 9-position should not alter the spectra significantly as well as its selectivity towards the selected metal ions. ${ }^{[26,110]}$ It was of greater interest to study the sensing abilities of the acridine derivatives substituted with different amines which is furthermore investigated in the next chapters.

\subsubsection{4,5-Bis $(N$-methyl- $N$-phenylaminemethylene)acridine (13)}

Some of the information written in this chapter has already been published in: A. Visscher, S. Bachmann, C. Schnegelsberg, T. Teuteberg, R. A. Mata and D. Stalke, Dalton Trans. 2016, 45, 5689-5699. ${ }^{[111]}$ 
Based on the information gained from preceded sensor systems, a more condensed amine is needed which does not exclude its own nitrogen atom from the coordination of metal ions. The most obvious solution is to omit additional donor groups at the side arms of the amine. A methyl group with its $+\mathrm{I}$ effect has already proven beneficial in the chapter before and should be retained. The second residue should be sterically demanding to increase the size-selectivity of the molecular sensor towards target cations. Additionally, more rigid side arms than $N, N^{\prime}, N^{\prime}-$ trimethylethylenediamine were favourable to prevent the formation of an oily product. This is why $N$-methyl- $N$-phenylamine was chosen as receptor unit. The rigid phenyl group with its ability to perform $\pi$ - $\pi$-interactions, as it was observed in the X-ray structure of $\mathbf{1 0}$, should contribute to obtain a solid compound.
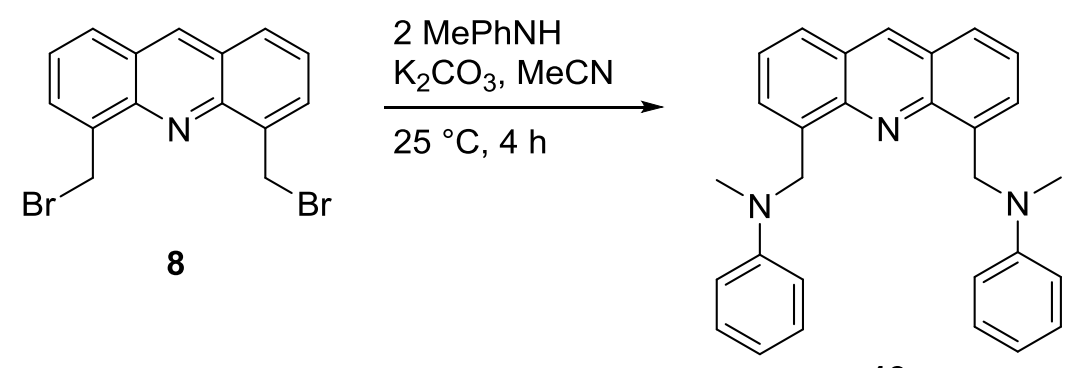

13

Scheme 2.18: Synthesis of the potential molecular sensor 4,5-bis( $N$-methyl- $N$-phenylaminemethylene)acridine (13).

The well-established starting material $\mathbf{8}$ was used again in acetonitrile with $\mathrm{K}_{2} \mathrm{CO}_{3}$ as base. Two equivalents of the amine were added and the reaction mixture was stirred for four hours at room temperature. The reaction progress can be monitored by different dissolving properties. In the beginning, 8 and $\mathrm{K}_{2} \mathrm{CO}_{3}$ are only partly soluble in MeCN forming a yellow suspension. At the end of the reaction, the product is completely dissolved and only the excess of salt added or formed during the reaction precipitates. The solvent was changed to ethyl acetate and washed several times with brine. The organic layer was dried over $\mathrm{MgSO}_{4}$, filtrated and the solvent was removed under reduced pressure. In the ${ }^{1} \mathrm{H}$ NMR spectrum only signals referring to the desired product and used solvents were observed. However, the fluorescence emission spectrum showed a huge, broad band at small wavelengths which is unusual for acridine derivatives. This band could be identified as the starting material $\mathrm{N}$-methylaniline. Its proton signals could then be assigned in the ${ }^{1} \mathrm{H} N M R$ spectrum (Figure 2.32), in particular by the comparison of the integral intensities.

Without being aware of the impurity, the signals of $N$-methylaniline can hardly be found in the spectrum. The signals of the phenyl protons of the starting material (marked with blue ellipsoids) are completely covered by the product signals. The signal of the methyl group at $2.67 \mathrm{ppm}$ is easily being mixed up with residual solvent signals. Solvent investigations showed that $\mathbf{1 3}$ is poorly soluble in methanol, slightly soluble in ethyl acetate and good soluble in tetrahydrofuran. Hence, the HNMePh amine is a liquid, a recrystallisation from methanol was performed and the impurities were filtrated off. 


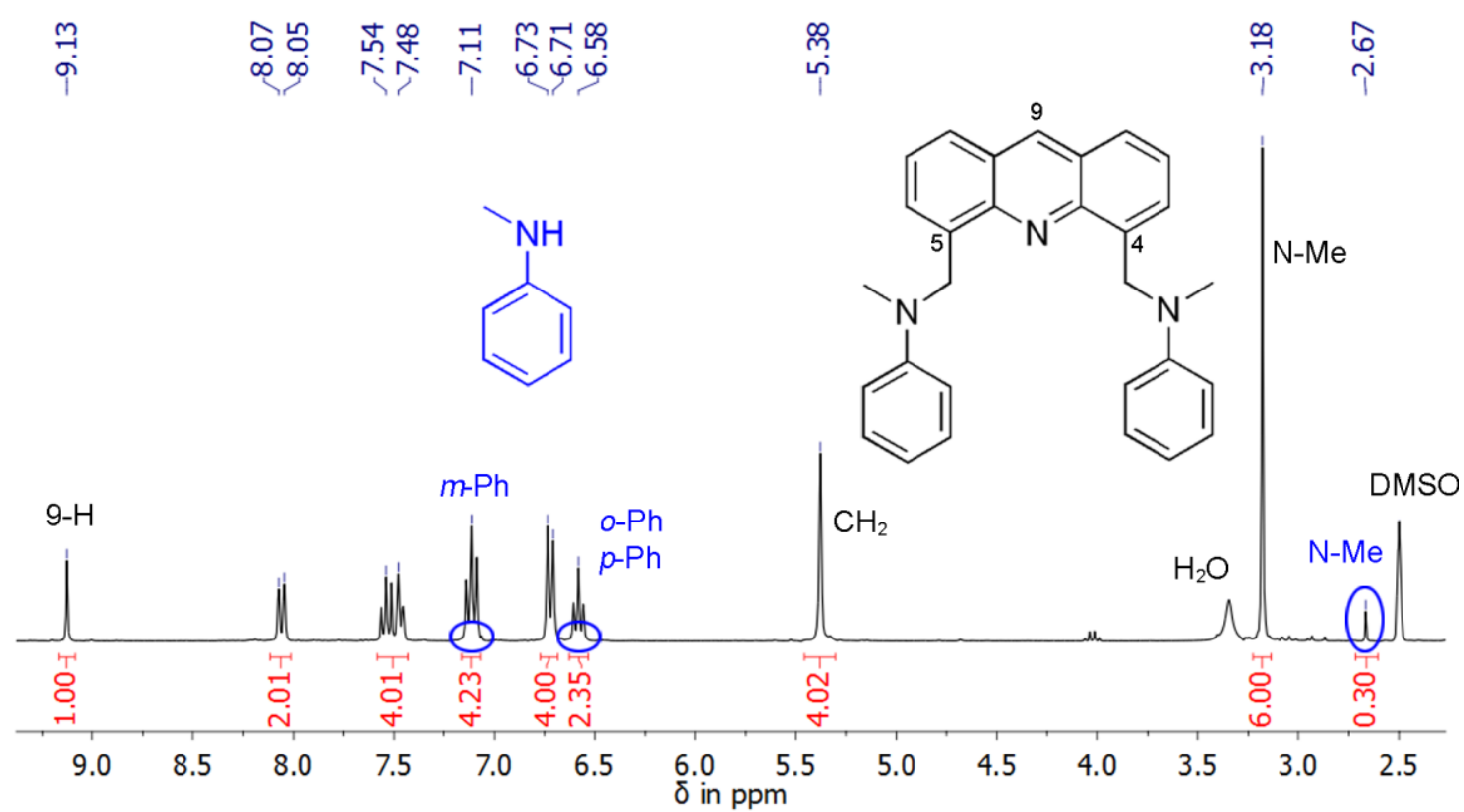

Figure 2.32: ${ }^{1} \mathrm{H}$ NMR spectrum of 13 in DMSO- $d_{6}$. The signals of $N$-methylaniline are covered by the signals of the product and are marked with blue ellipsoids.

\subsubsection{Luminescence properties of 4,5-bis $(N$-methyl- $N$-phenylaminemethylene)- acridine (13)}

In comparison to the investigated compounds $\mathbf{1 0}$ and 11, the acridinyl amine $\mathbf{1 3}$ has less donating groups. Only the two nitrogen atoms of the amines at the 4- and 5-position trigger the PET effect. This could be an advantage according to the efficiency of the off/on switch (see discussion to Figure 2.31). The commonly used irradiation wavelength of $357 \mathrm{~nm}$ for the fluorescence measurements did not result in any significant emission of light. Therefore, an absorption spectrum of the ligand solution in methanol was recorded (Figure 2.33).
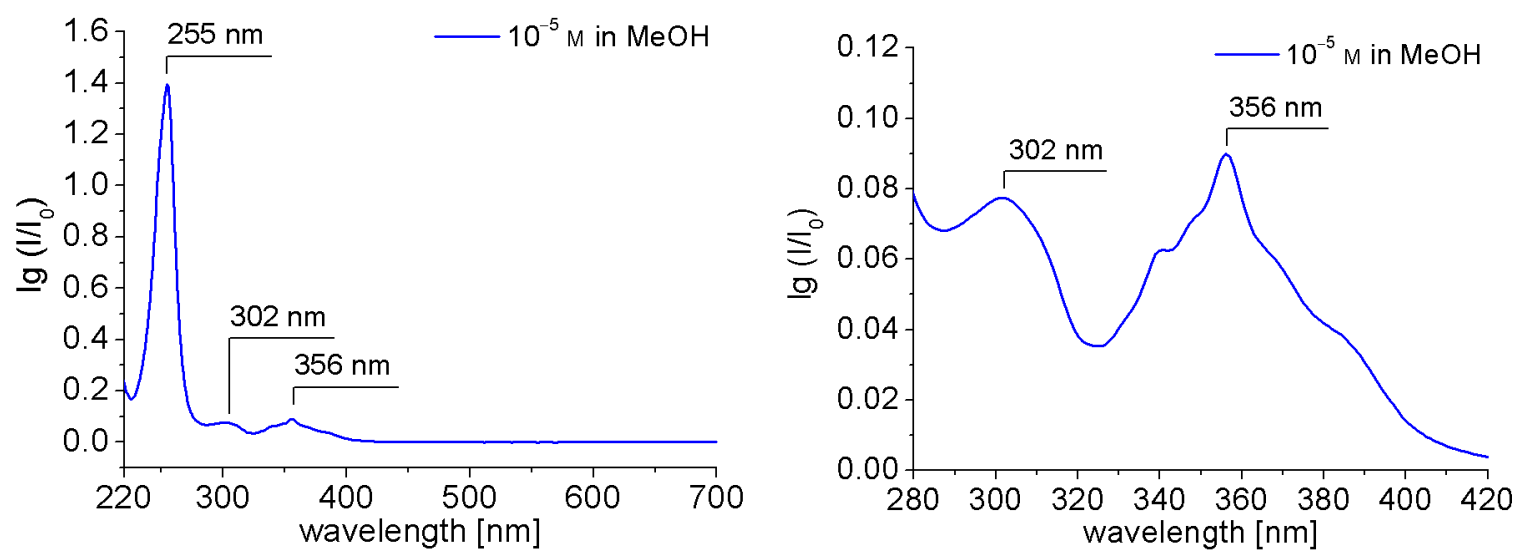

Figure 2.33: UV/vis absorption spectrum of 13 in $\mathrm{MeOH}\left(10^{-5} \mathrm{M}\right)$ showing the complete wavelength range (left) and the significant wavelength region in detail (right).

The absorption spectrum is corrected by subtraction of a methanol reference spectrum. The experiment yielded three observable signals with a large and narrow one at $255 \mathrm{~nm}$, a broad signal at $302 \mathrm{~nm}$ and a slightly structured signal at $356 \mathrm{~nm}$. The two highest energy transitions at 255 
and $356 \mathrm{~nm}$ are $\pi-\pi^{*}$ in nature and are referred to the acridine backbone. ${ }^{[12]}$ The broad band at $302 \mathrm{~nm}$ was not determined. The experiment shows that the same excitation wavelength which was used before can be applied to 13, too. The absence of any fluorescence emission demonstrates the excellent quenching mechanism of the PET.

In Figure 2.34, left, both excitation and emission spectra of $\mathbf{1 3}$ in methanol are depicted. Apart from the Raman scattering of the solvent at $398 \mathrm{~nm}$ including the two smaller bands before and after ${ }^{[100]}$ no significant emission of light can be observed. However, this positive result concerning the PET effect was slightly diminished by the next experiment. Aqueous $\mathrm{HCl}$ was added to the cuvette to protonate the amine groups and prevent them from fluorescence quenching. Similar to the acridine derivatives investigated before, the emission intensity only rises after an excess of $\mathrm{HCl}$ was added to the sample (Figure 2.34, right).
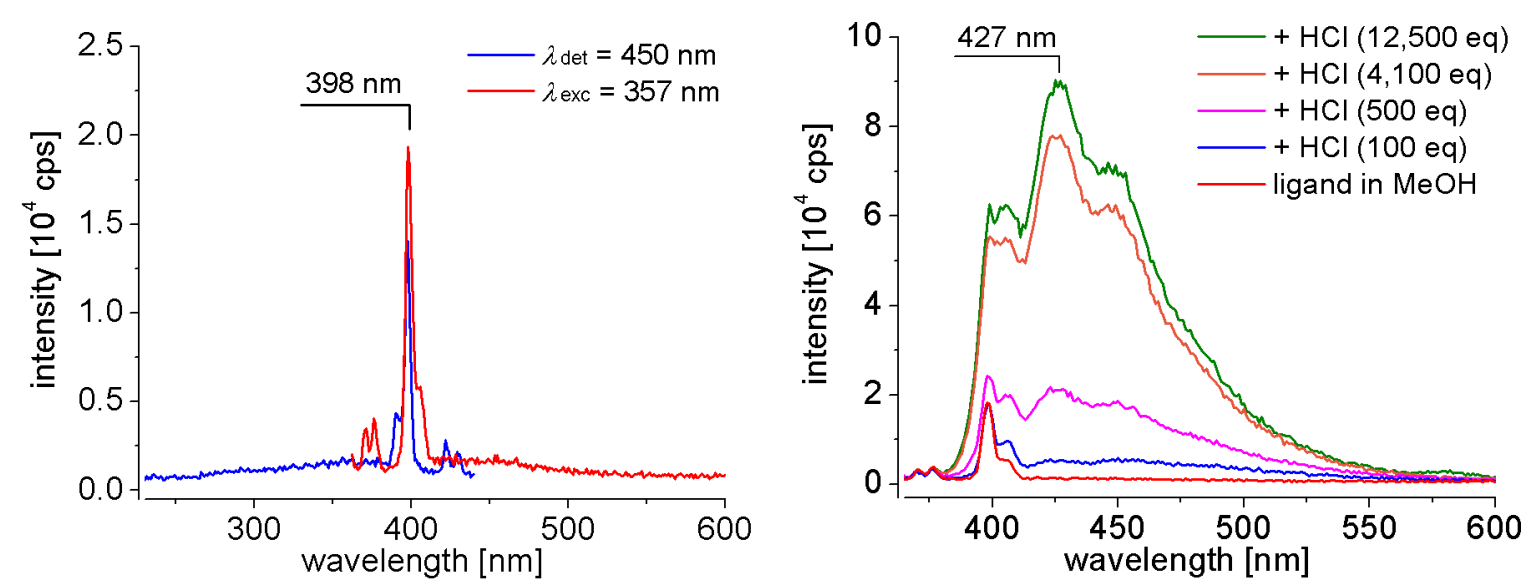

Figure 2.34: Left: Excitation (blue: $\lambda_{\mathrm{det}}=450 \mathrm{~nm}$ ) and emission (red: $\lambda_{\mathrm{exc}}=357 \mathrm{~nm}$ ) spectrum of $\mathbf{1 3}$ in $\mathrm{MeOH}\left(10^{-5} \mathrm{M}\right)$; right: emission spectra of $\mathbf{1 3}$ in $\mathrm{MeOH}$ with addition of aqueous $\mathrm{HCl}\left(\lambda_{\text {exc }}=357 \mathrm{~nm}\right)$.

An excitation spectrum recorded afterwards now shows the typical curve with the highest band at $357 \mathrm{~nm}$ (Figure 2.35). $\mathbf{1 3}$ seems to behave similar to $\mathbf{1 1}$ which responded to the presence of zinc and cadmium ions. A full fluorescence screening was therefore performed on this new compound.

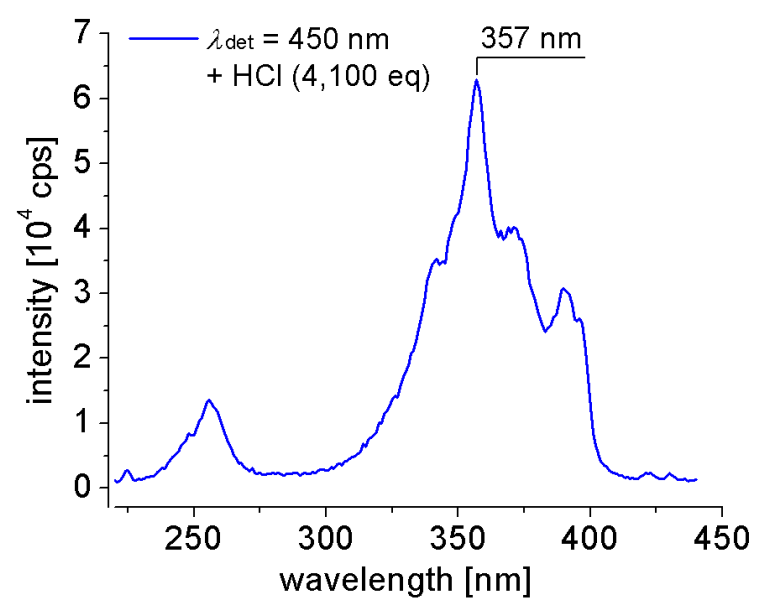

Figure 2.35: Excitation spectrum of $\mathbf{1 3}$ in methanol $\left(\lambda_{\mathrm{det}}=450 \mathrm{~nm}, 10^{-5} \mathrm{M}\right)$ with addition of aqueous $\mathrm{HCl}$. 


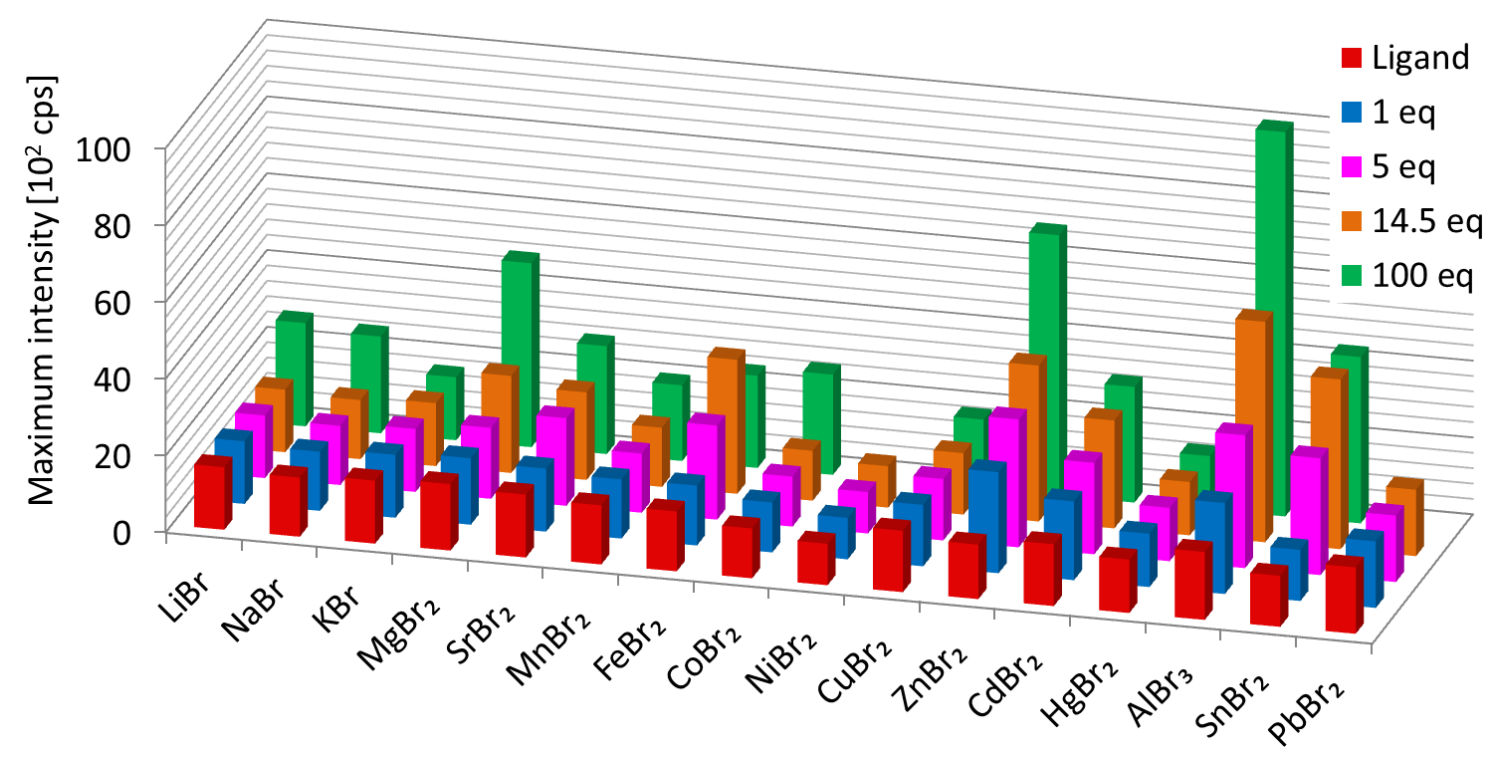

Figure 2.36: Cation screening of 13 in methanol $\left(10^{-5} \mathrm{M}, \lambda_{\mathrm{exc}}=357 \mathrm{~nm}\right)$, with a metal salt concentration ranging from 1 to 100 equivalents. The maximum emission intensity is depicted for each metal ion addition.

The three dimensional bar chart in Figure 2.36 illustrates the low response of $\mathbf{1 3}$ to the tested salts. Only a small enhancement of the intensity is observed after the addition of 100 equivalents of $\mathrm{ZnBr}_{2}$ or $\mathrm{AlBr}_{3}$. However, this is not a significant response in contrast to the results of $\mathbf{1 1}$ in Figure 2.25. By looking at the emission spectra of the mentioned metal salts, two different wavelengths are observed (Figure 2.37). The maximum for $\mathrm{ZnBr}_{2}(456 \mathrm{~nm})$ is in line with the studies of the former acridine derivatives. The titration of $\mathrm{AlBr}_{3}$, however, led to a hypsochromic shift of the emission maximum to $427 \mathrm{~nm}$. This value is known for the protonated ligand as can be seen in Figure 2.34, right. The aluminium ion is a Lewis acid which is able to make the solvent release protons which in turn protonates the organic ligand. The same observations were made with $\mathrm{SnBr}_{2}$ to a lesser extent.
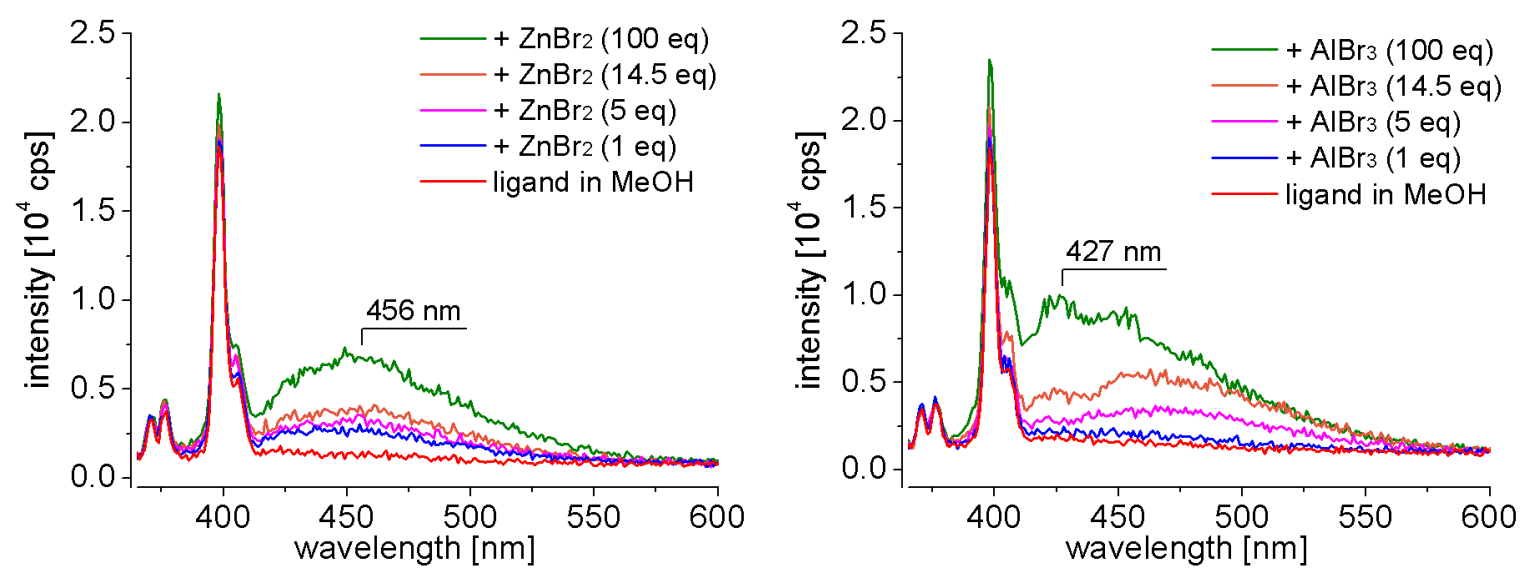

Figure 2.37: Emission spectra of $\mathbf{1 3}$ in $\mathrm{MeOH}\left(10^{-5} \mathrm{M}, \lambda_{\mathrm{exc}}=357 \mathrm{~nm}\right)$ with addition of $\mathrm{ZnBr}_{2}$ (left) and $\mathrm{AlBr}_{3}$ (right).

These results clearly indicate that the lone pairs of the nitrogen atoms only show a weak basicity. On account of the aniline like structure, the lone pair of the nitrogen atom is partly delocalised into the phenyl ring which results in a lower basicity $\left(+\mathrm{M}\right.$ effect). ${ }^{[113]}$ This is confirmed by the large excess of hydrochloric acid which was needed to achieve an enhancement of the emission 
intensity. The shielding of the metal ions by the methanol molecules seems to be stronger than the chelating ability of $\mathbf{1 3}$. This is why the aprotic, less polar solvent dichloromethane was used for further experiments.
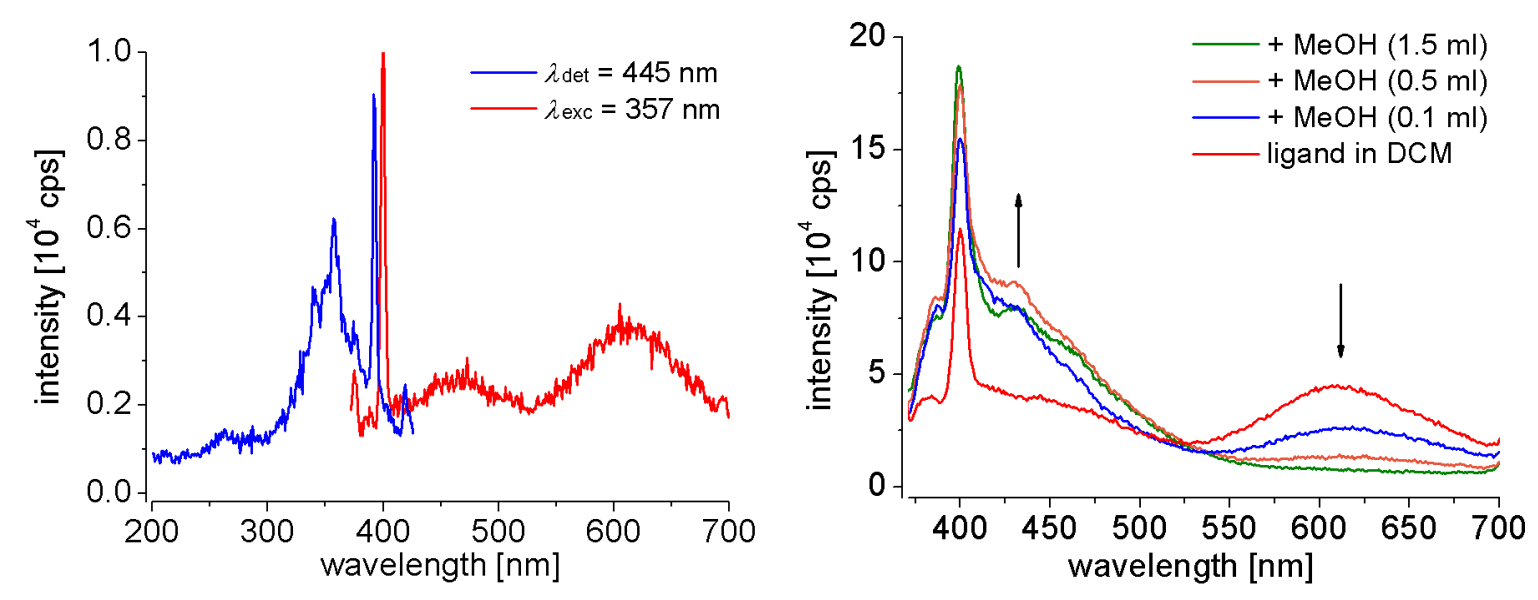

Figure 2.38: Left: Excitation (blue: $\lambda_{\operatorname{det}}=445 \mathrm{~nm}$ ) and emission (red: $\lambda_{\mathrm{exc}}=357 \mathrm{~nm}$ ) spectrum of 13 in DCM $\left(10^{-5} \mathrm{M}\right)$; with the usual front and exit slit bandpass of $2 \mathrm{~nm}$; right: the same emission spectrum of 13 but with a bandpass of $4 \mathrm{~nm}$ and with addition of pure methanol.

As can be seen in Figure 2.38, left, the excitation and emission spectrum of $\mathbf{1 3}$ in DCM is very low in its intensity. But in contrary to the measurements in methanol, a rough curve can be observed. Unusual for the emission spectrum is the existence of two separate bands; one in the expected region around $470 \mathrm{~nm}$ and the other at longer wavelengths of $610 \mathrm{~nm}$. This lower energy hints to the formation of excimers which was already mentioned in Chapter 2.2.6. Excimers are usually found in glasses at very low temperatures. In solution, this is only observable in non-polar solvents at specified concentrations. That is why the addition of polar methanol (Figure 2.38, right) reduces the band at $610 \mathrm{~nm}$ along with an increase of intensity at the short wavelength region.
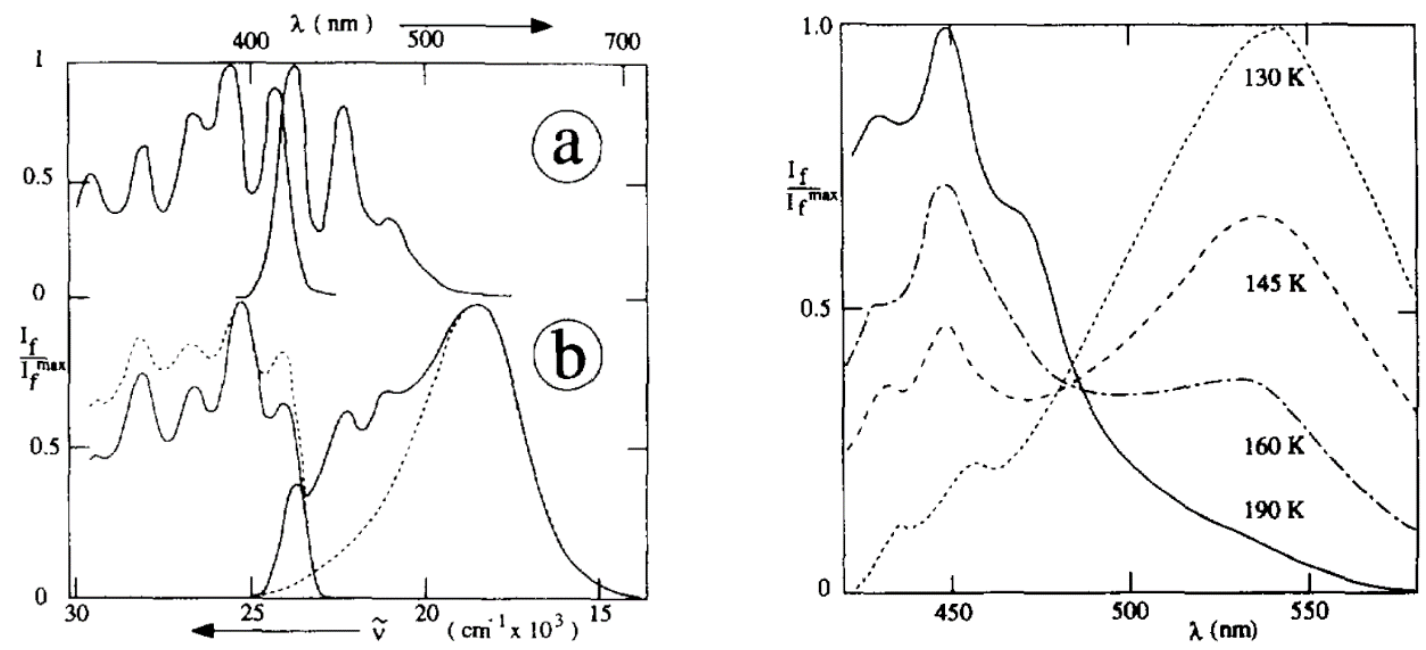

Figure 2.39: Left diagram: Fluorescence excitation (left) and emission (right, $\lambda_{\mathrm{exc}}=353 \mathrm{~nm}$ ) spectra of 9phenyl-2,10-diazaanthracene at $77 \mathrm{~K}$ in $\mathrm{MCH}\left(0.02 \mathrm{mM} ; \lambda_{\operatorname{det}}=450 \mathrm{~nm}\right)$ (a) and in D-P $(0.12$ and $0.45 \mathrm{mM}$, full and broken lines respectively; $\lambda_{\text {det }}=580 \mathrm{~nm}$ ) (b); right diagram: Fluorescence spectra (non-corrected) for 8-methyl-9-phenyl-2,10-diazaanthracene in D-P at 130, 145, 160 and $190 \mathrm{~K}$; MCH: methylcyclohexane; D-P: 2,2-dimethylbutane/n-pentane (8:3). ${ }^{[14]}$ 
Excited-state dimers of acridine systems are rarely present in literature. Nikolov and Görner studied this phenomenon with an diazaanthracene derivative. ${ }^{[14]}$ In Figure 2.39, they show both the concentration and temperature dependency of its excimer formation. The concentration dependency is investigated in non-polar glasses at $77 \mathrm{~K}$. At a concentration of $0.12 \mathrm{mM}$, both the monomer and the excimer species are observed (b). At almost quadrupled concentration, only the fluorescence emission of the excimer is detectable. The same is true for varying temperature experiments. In the right diagram, the temperature dependency is illustrated in a non-polar solution. Here, the ratio of monomer/excimer decreases with increasing temperature. When the excimer formation is not visible anymore, the temperature reaches $190 \mathrm{~K}$. This is still a very low value as the experiments in this thesis were carried out at $295 \mathrm{~K}$. It is therefore a rarely phenomenon under these measurement conditions.

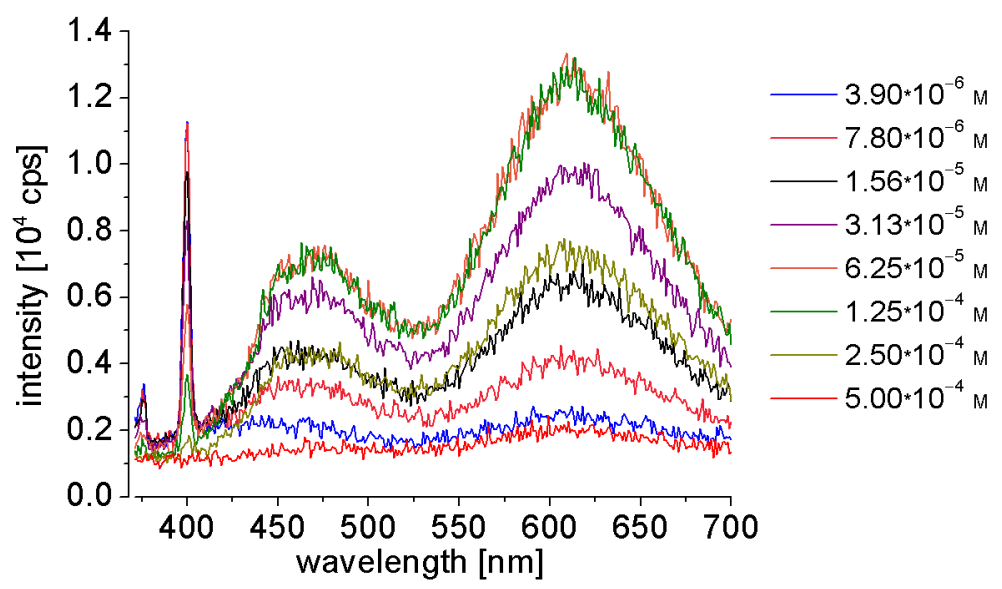

Figure 2.40: Emission spectra of 13 in $\operatorname{DCM}\left(\lambda_{\mathrm{exc}}=357 \mathrm{~nm}\right)$ at different concentrations.

In Figure 2.40, a dilution experiment in DCM was performed to examine if various concentrations of 13 influence the emission spectrum significantly. The experiment was started with a concentration of $5.0 \cdot 10^{-4} \mathrm{M}$ and was then diluted by half several times. Higher concentrations only led to absorption of the incident beam. During the experiment, different intensity ratios of the two bands were observed. At high or low concentrations, the monomer/excimer ratio is about $1: 1$, whereas at $1.25 \cdot 10^{-4} \mathrm{M}$ and $6.25 \cdot 10^{-4} \mathrm{M}$ the intensity of the assumed excimer rises. Both experiments, the concentration dependency and the varied solvent polarity should verify the existence of the described phenomenon. The question how the ligand responds to $\mathrm{Zn}^{2+}$ ions in a non-polar solvent will be discussed in the following.

In Figure 2.41, left, an increase in intensity after the addition of $\mathrm{ZnBr}_{2}$ is readily observable. Furthermore, the shape and the emission wavelength alters during the titration. A very broad band is observed in the beginning when one equivalent of the metal ion is present in solution. With five equivalents present, four significant peaks are observable. Another ten equivalents lead to the formation of a single band at $470 \mathrm{~nm}$. When 100 equivalents of zinc ions are added, the shape of the curve remains the same but is lowered in intensity due to dilution. According to these observations, it is possible that two metal complexes which emit light at different wavelengths are formed with small amounts of $\mathrm{ZnBr}_{2}$ added. With further addition (around $15 \mathrm{eq}$ ), the complex emitting at $470 \mathrm{~nm}$ is favoured. In contrary to the emission spectra measured before, no band could be observed at higher wavelengths (> $550 \mathrm{~nm}$ ). 

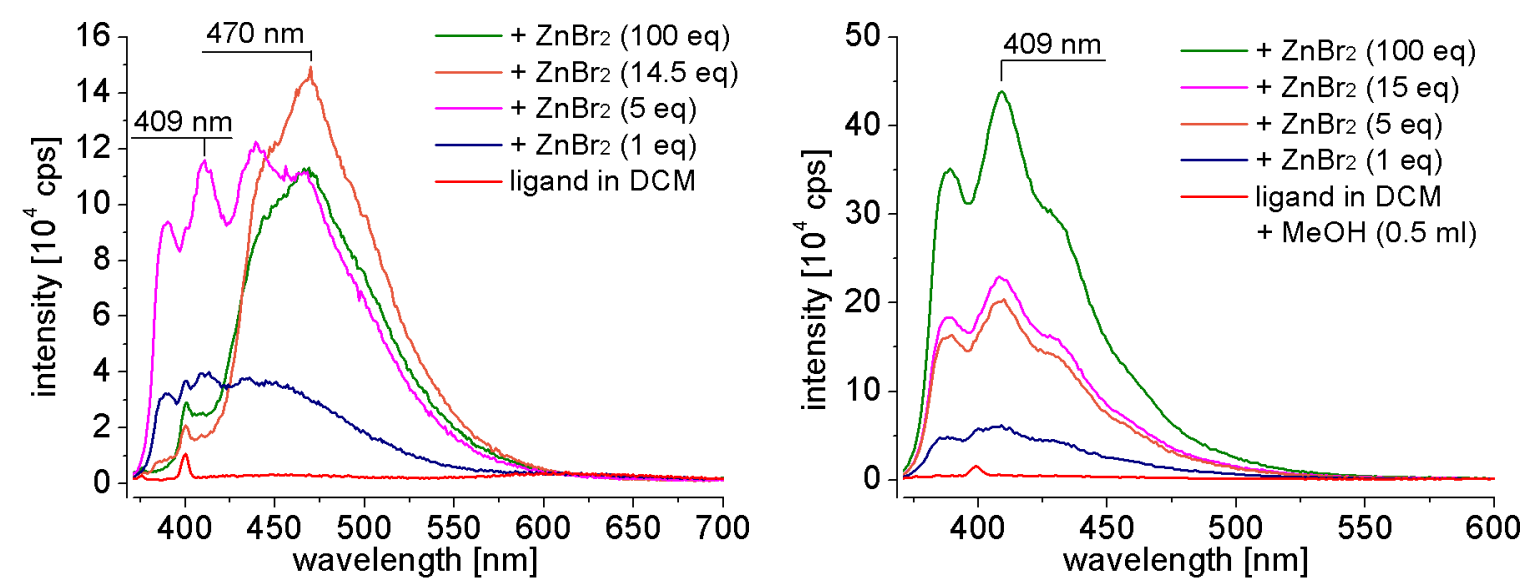

Figure 2.41: Emission spectra of 13 in $\mathrm{DCM}\left(10^{-5} \mathrm{M}, \lambda_{\mathrm{exc}}=357 \mathrm{~nm}\right)$ with addition of $\mathrm{ZnBr}_{2}$. In the right diagram, methanol was added before $\mathrm{ZnBr}_{2}$ was titrated to the sample.

Since the polarity of the solvent strongly influences the behaviour of the synthesised molecular sensor, another experiment with $\mathrm{ZnBr}_{2}$ was performed. Half a millilitre methanol was added to the free ligand in DCM before the solution was titrated with the metal ion (Figure 2.41, right). In this case, only the structured band at $409 \mathrm{~nm}$ was observed - independent of the added amount of zinc ions. These experiments suggest that the band at $470 \mathrm{~nm}$ in pure DCM results from an excimer complex containing one or more zinc ions. The latter is more likely since the band at higher wavelengths is only favoured when an excess of metal ions is present.

Similar investigations with a pyrene based sensor were made by $W u$ et al. in 2012. ${ }^{[115]}$ They combined the nucleobase thymine, working as a $\mathrm{Hg}^{2+}$ ion receptor, with the fluorescence dye pyrene, which is known to form intra- and intermolecular excimers. ${ }^{[16]}$ The publication describes a strong bathochromic shift of almost $100 \mathrm{~nm}$ of the fluorescence emission upon addition of mercury ions (Figure 2.42).
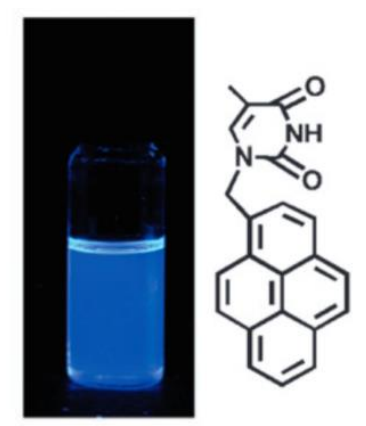

Monomer (РyT), emission at $378 \mathrm{~nm}$
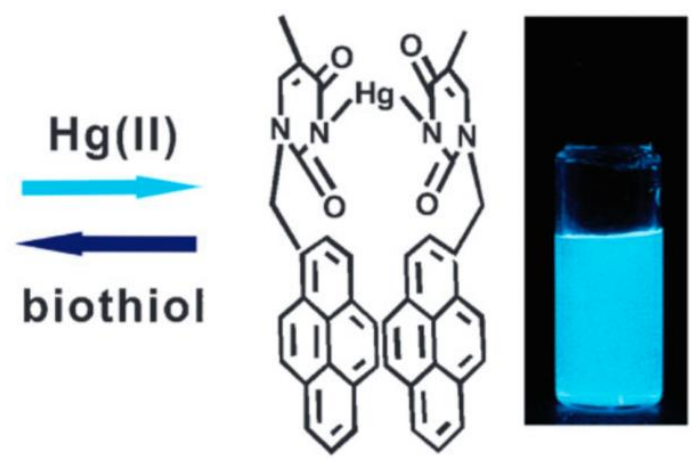

\section{Excimer (PyT-Hg(II)-TPy), emission at $473 \mathrm{~nm}$}

Figure 2.42: Illustration of the proposed sensing mechanism of PyT (pyrene-thymine) in the presence of $\mathrm{Hg}^{2+}$ ions. The process is reversible by the addition of biothiol (glutathione). The measurements were performed in a HEPES buffered water/DMSO mixture (7:1, $\mathrm{pH} 7.0) .^{[115]}$

The results of $W u$ et al. are similar to those described here and could explain the observations made by the fluorescence experiments. Although the results obtained by the use of DCM were interestingly, this solvent was not in the focus of this thesis. As already mentioned in Chapter 
2.2.9, neither the comparability with other metal ions nor the similarity to the biological important solvent water is given with DCM.

\subsubsection{4,5-Bis $(N$-methyl- $N$-phenylaminemethylene)acridine hydrochloride (14)}

Several attempts to crystallise $\mathbf{1 3}$ were not successful. That is why it was precipitated as hydrochloride salt, since the saline ionic character can support the formation of crystalline material. 13 was suspended in ethanol and an excess of conc. aqueous $\mathrm{HCl}$ was added. The reaction mixture was stirred at room temperature for $30 \mathrm{~min}$ whereby it became a clear solution. The crude product was extracted three times with hexane, then the volatile compounds were removed under reduced pressure.<smiles>CN(Cc1cccc2cc3cccc(CN(C)c4ccccc4)c3nc12)c1ccccc1</smiles>

13

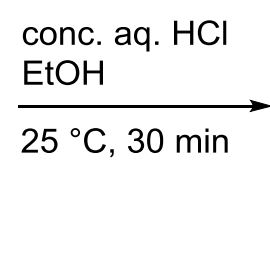<smiles>CN(Cc1cccc2cc3cccc(CNc4ccccc4O)c3nc12)c1ccccc1</smiles>

14

Scheme 2.19: Hydrochlorination of the amine functionalities led to the formation of the saline $\mathbf{1 4}$.

The different analytical methods were not conclusive in all cases. The elemental analysis gave results which did not allow to distinguish if two or three chloride anions are present. A third chloride would assume the protonation of the acridine nitrogen atom as well. Unfortunately, only an EI mass spectrum was recorded which showed $\mathrm{m} / \mathrm{z}$ fragments without the significant chlorine patterns. This can be attributed to the hard ionisation technique. Other techniques like electrospray ionisation (ESI) or fast atom bombardment (FAB) yield lower fragmentation rates and would have been more suitable for the identification of the saline compound.

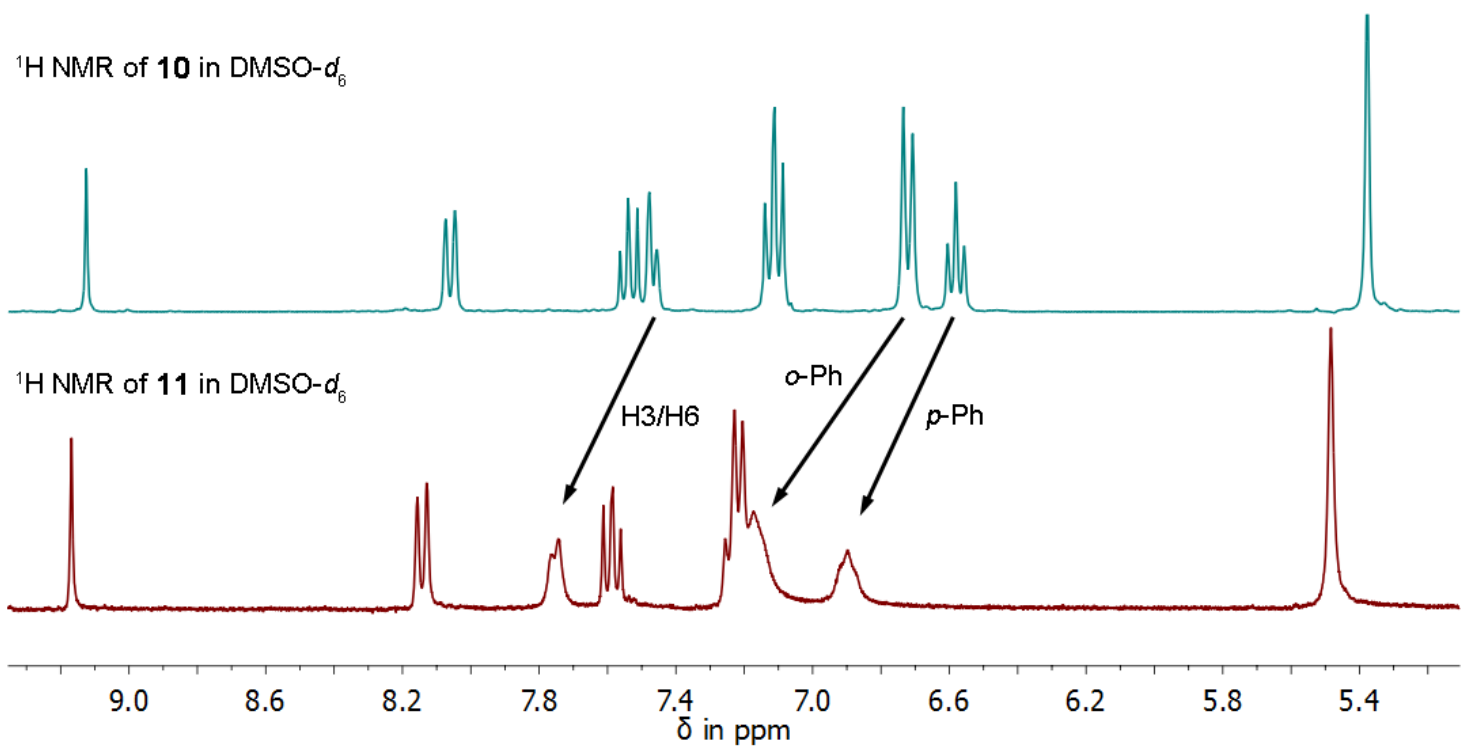

Figure 2.43: Comparison of the chemical shifts of 13 (top) and 14 (bottom) of their ${ }^{1} \mathrm{H}$ NMR spectra. 
The investigation of $\mathbf{1 4}$ by NMR spectroscopy gave a good hint towards the protonation of the amines. All proton signals are shifted to lower magnetic fields (Figure 2.43) whereas some of the signals are broadened and show a stronger shift (marked with arrows). These signals belong to the protons of the phenyl groups and of the acridine ring at $\mathrm{C} 3 / \mathrm{C} 6$. The latter are the neighboured positions of the methylene spacer and therefore more effected than the other aromatic protons. These results from the NMR spectrum are in line with the proposed synthesis of $\mathbf{1 4}$ in Scheme 2.19 , although no $\mathrm{NH}$ proton was visible. This is mainly due to a rapid exchange of acidic protons with the used DMSO- $d_{6}$ or with residual water in the NMR solvent. ${ }^{[117]}$ The latter is very likely, since the water signal is shifted by $0.6 \mathrm{ppm}$. The broadened signals could be explained by the presence of dynamic effects in solution. ${ }^{[118]}$ The chlorides act as counter ions to the aminium cations and are in close proximity to them. If they exchange slowly enough, the dynamic effect could be monitored by NMR spectroscopy.

Besides the spectroscopic analysis, the aim of the synthesis of the saline $\mathbf{1 4}$ was the crystallisation of the compound to compare its geometry with the similar arranged sensor ligands. The crystallisation was realised in an equivalent mixture of ethanol/hexane, in which the $\mathrm{HCl}$ adduct was dissolved at boiling temperature. After cooling to room temperature, the solution was stored for four weeks. During this time, suitable crystals for X-ray analysis formed (Figure 2.44).

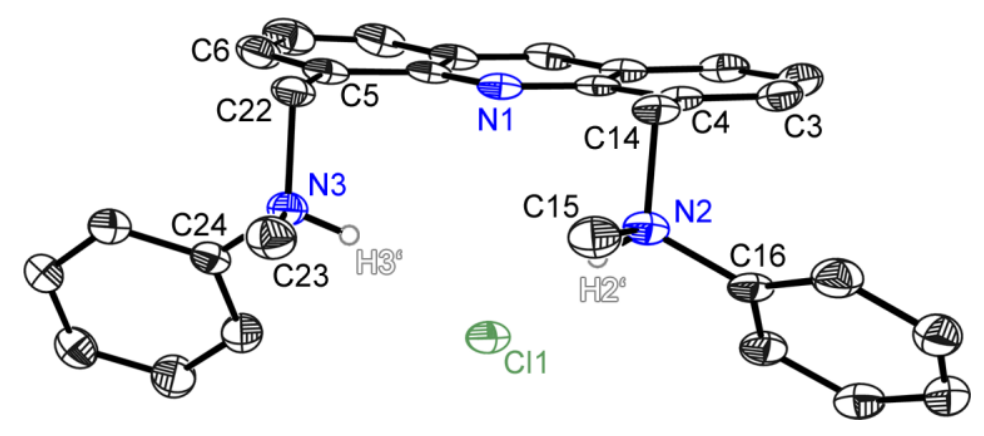

Figure 2.44: Solid state structure of $\mathbf{1 4}$ crystallised from an ethanol/hexane mixture. Not freely refined hydrogen atoms, the disordered solvent molecules $\left(\mathrm{EtOH}\right.$ and $\left.\mathrm{H}_{3} \mathrm{O}^{+}\right)$, and two more chloride ions were omitted for clarity.

In the above figure the asymmetric unit of $\mathbf{1 4}$ is depicted, showing the organic ligand with two protons attached to the amine side arms. The disordered solvent molecules $\left(\mathrm{EtOH}\right.$ and $\mathrm{H}_{3} \mathrm{O}^{+}$) as well as two more chloride ions were omitted in this illustration. However, these additional molecules can explain the inconsistent values of the elemental analysis. The comparison of this data is listed in the experimental settings. The compound crystallises in the monoclinic space group $C 2 / c$ and the receptor side arms are arranged almost symmetrical. Both positions of the hydrogen atoms of the amines (H2' and H3') were refined freely. They point towards the centred $\mathrm{Cl} 1$ atom forming hydrogen bonds which will be discussed in the following section. First, the packed structure was studied to determine further hydrogen-chlorine interactions as well as the already discussed packing effects between two acridine or phenyl systems. In comparison to 10, the phenyl rings in this structure do not show any $\pi-\pi$-interactions. Here, $\mathrm{CH} \cdots \pi$ interactions between a hydrogen atom (H23C) of the methyl group C23 and the phenyl ring of $\mathrm{N} 2$ are present with a distance of $261.2 \mathrm{pm}$. Additionally, the acridine backbones get close to each other, resulting in a short $\pi$ - $\pi$-distance of $338.9 \mathrm{pm}$. This value is $0.7 \mathrm{ppm}$ smaller than the distance in 
the packed structure 10. The overlap of the acridine ring systems is shown in Figure 2.45. These different types of interactions play a crucial role for the arrangement of the crystal structure.

The $\mathrm{N}-\mathrm{C}$ bond lengths listed in Table 2.7 are significantly elongated compared to typical bond lengths of $\mathrm{N}\left(\mathrm{sp}^{3}\right)-\mathrm{C}\left(\mathrm{sp}^{3}\right)$ and $\mathrm{N}\left(\mathrm{sp}^{3}\right)-\mathrm{C}\left(\mathrm{sp}^{2}\right)$ that are $147 \mathrm{pm}$ and $143 \mathrm{pm}$, respectively. ${ }^{[84]}$ Here, the longest bond is N3-C22 with 152.7(3) pm. Compared to the relative acridine amine 10, the highest deviation is $6.2(5) \mathrm{pm}$. The bonds between the nitrogen atoms and the ipso-C of the phenyl groups are as long as a bond to an $\mathrm{sp}^{3}$ hybridised carbon atom (both are 147.9(3) pm). These values could be attributed to the present tertiary ammonium ions. The nitrogen atoms N2 and $\mathrm{N} 3$ are positively charged through the protonation resulting in a smaller electronegativity value and less attraction to the carbon atoms. This leads to the elongation of the $\mathrm{N}-\mathrm{C}$ bonds.

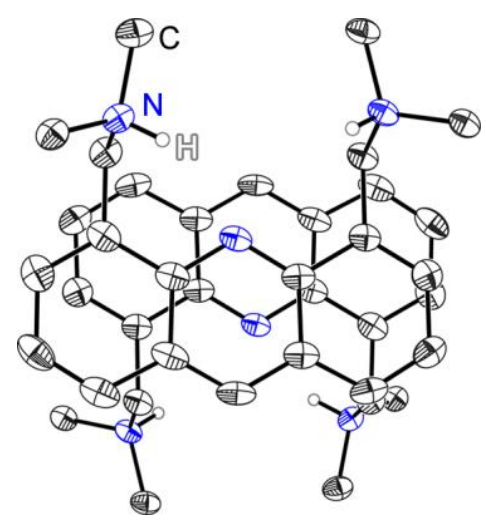

Figure 2.45: Detail of the $\pi$ - $\pi$-overlap of the acridine moieties of $\mathbf{1 4}$.
Table 2.7: Selected bond lengths and angles of $\mathbf{1 4}$.

\begin{tabular}{|c|c|c|c|}
\hline \multicolumn{2}{|l|}{ Bond [pm] } & \multicolumn{2}{|l|}{ Angle $\left[{ }^{\circ}\right]$} \\
\hline $\mathrm{N} 2-\mathrm{C} 14$ & $152.5(3)$ & $\mathrm{C} 4-\mathrm{C} 14-\mathrm{N} 2$ & $112.77(19)$ \\
\hline $\mathrm{N} 2-\mathrm{C} 15$ & $150.4(3)$ & C14-N2-C15 & $109.16(19)$ \\
\hline $\mathrm{N} 2-\mathrm{C} 16$ & $147.9(3)$ & $\mathrm{C} 14-\mathrm{N} 2-\mathrm{C} 16$ & $112.46(19)$ \\
\hline $\mathrm{N} 3-\mathrm{C} 22$ & $152.7(3)$ & C15-N2-C16 & $113.55(19)$ \\
\hline $\mathrm{N} 3-\mathrm{C} 23$ & $149.2(3)$ & $\mathrm{C} 5-\mathrm{C} 22-\mathrm{N} 3$ & $109.6(2)$ \\
\hline $\mathrm{N} 3-\mathrm{C} 24$ & 147.9(3) & $\mathrm{C} 22-\mathrm{N} 3-\mathrm{C} 23$ & $110.78(19)$ \\
\hline \multirow[t]{4}{*}{$\mathrm{N} 1 \cdots \mathrm{Cl} 1$} & 332.7 & $\mathrm{C} 22-\mathrm{N} 3-\mathrm{C} 24$ & $113.61(19)$ \\
\hline & & $\mathrm{C} 23-\mathrm{N} 3-\mathrm{C} 24$ & $111.4(2)$ \\
\hline & & $\mathrm{C} 3-\mathrm{C} 4-\mathrm{C} 14-\mathrm{N} 2$ & $-89.5(3)$ \\
\hline & & $\mathrm{C} 6-\mathrm{C} 5-\mathrm{C} 22-\mathrm{N} 3$ & $97.1(3)$ \\
\hline
\end{tabular}

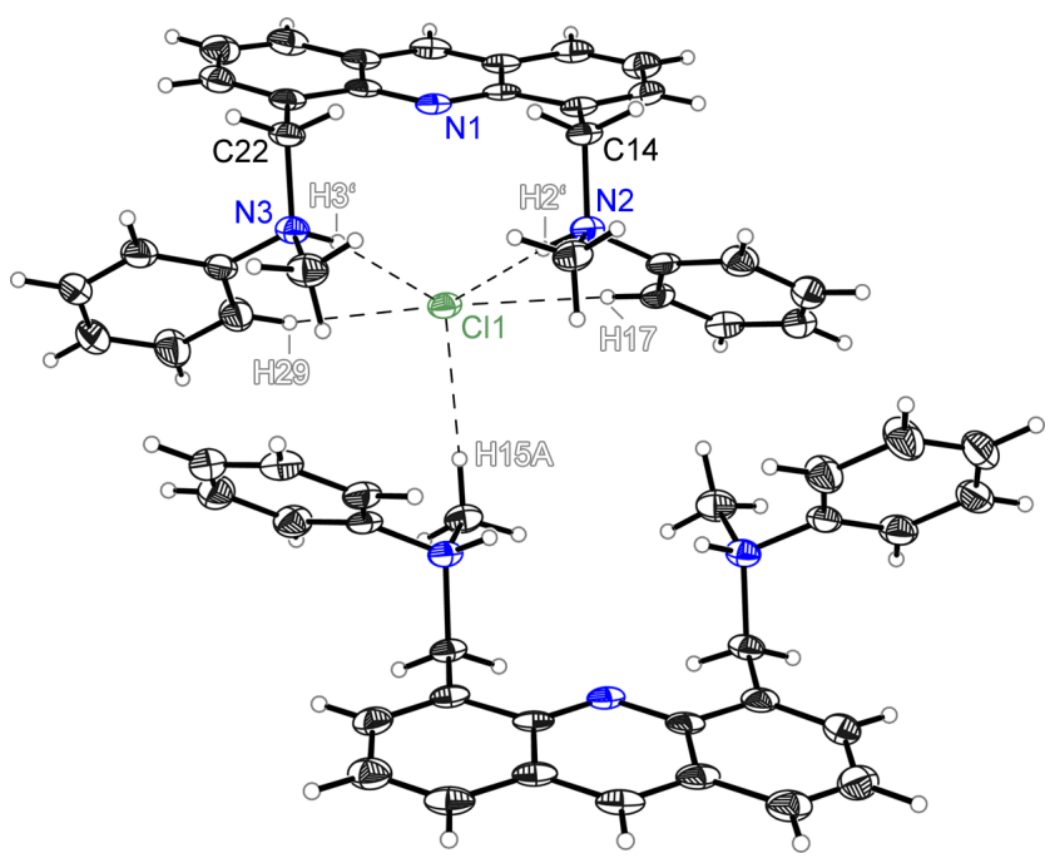

Figure 2.46: Two molecules of $\mathbf{1 4}$ demonstrating the hydrogen bonds to the centred chlorine atom $\mathrm{Cl1}$. 
The usually tetrahedral $\mathrm{C}-\mathrm{N}-\mathrm{C}$ angles are likewise affected. The bonded hydrogen atom is sterically less demanding than the former lone pair, leading to larger angles (e.g. C23-N3-C24: $\left.113.61(19)^{\circ}\right)$. The torsion angles of the receptor side arms are very close to the perpendicular angle with values of $89.5(3)^{\circ}$ and $97.1(3)^{\circ}$. This is probably due to the formation of hydrogen bonds between the ammonium ions and the chloride.

In Figure 2.46, the environment of $\mathrm{Cl} 1$ is illustrated, including five hydrogen-chloride interactions which are marked with dashed lines. Two of them have a nitrogen atom as donor (D) and are stronger than the hydrocarbon counterparts. Nitrogen atoms possess a higher electronegativity than carbon atoms, forming partially positive charged hydrogen atoms. This results in a stronger interaction with the acceptor (A). According to the literature, DH $\cdots$ A interactions can be stated as hydrogen bonds if they are shorter than $320 \mathrm{pm}$ and have a greater DHA angle than $90^{\circ} .{ }^{[89]}$ When comparing the values for the $\mathrm{NH} \cdots \mathrm{Cl}$ interaction with the defining parameters in Table 2.8 , the interactions can be classified as moderate hydrogen bonds. The $\mathrm{H} \cdots \mathrm{Cl}$ distances are quite long with values of 216(2) and 224(2) pm for $\mathrm{H}^{\prime} \cdots \mathrm{Cl} 1$ and $\mathrm{H} 3^{\prime} \cdots \mathrm{Cl} 1$, respectively. The first parameter can be stated as a moderate interaction, whereas the second distance lies in the range for weak hydrogen bonds. The DHA angles are large with values of 174(2) and 163(2) ${ }^{\circ}$, respectively. The first angle can be attributed to a strong interaction with an almost linear arrangement. Referring to these properties, the $\mathrm{H} 2^{\prime} \cdots \mathrm{Cl} 1$ interaction is significantly stronger than its $\mathrm{H} 3$ ' counterpart. Another classification can also be gained by the comparison of the values with the data of the diagram, depicted in Figure 2.47. Here, $\mathrm{NH}_{3}{ }^{+} \ldots \mathrm{Cl}^{-}$interactions are illustrated in an angular scatter plot of $\mathrm{N}-\mathrm{H} \cdots \mathrm{Cl}$ angles against $\mathrm{H} \cdots \mathrm{Cl}$ distances. The values of the $\mathrm{N}-\mathrm{H} \cdots \mathrm{Cl}$ interactions are marked in red in the figure, assuming strong bonding situations in comparison to the relative literature values demonstrated as black dots.

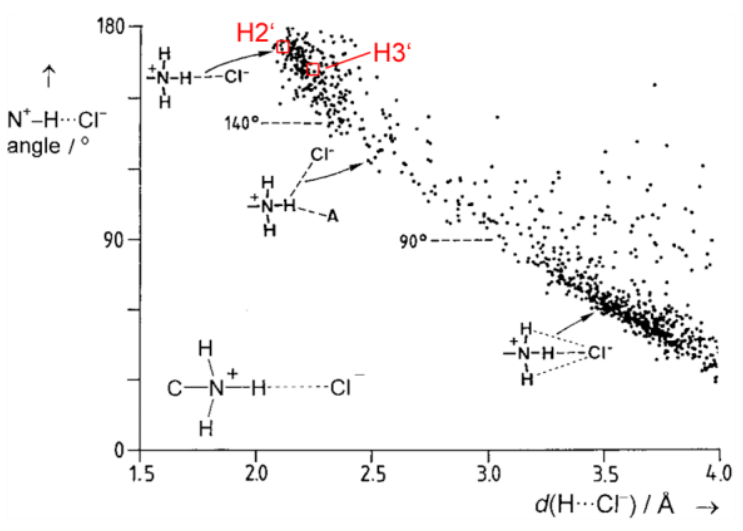

Table 2.8: Part of the classification of hydrogen bonds after Jeffrey. ${ }^{[120]}$ Distances are given in $[\mathrm{pm}]$ and angles in $\left[^{\circ}\right]$.

\begin{tabular}{cccc}
\hline & Strong & Moderate & Weak \\
\hline \hline $\begin{array}{c}\text { interaction } \\
\text { type }\end{array}$ & $\begin{array}{c}\text { strongly } \\
\text { covalent }\end{array}$ & $\begin{array}{c}\text { mostly } \\
\text { electrost. }\end{array}$ & $\begin{array}{c}\text { electrost./ } \\
\text { dispers. }\end{array}$ \\
$d(\mathrm{H} \cdots \mathrm{A})$ & $120-150$ & $150-220$ & $>220$ \\
$d(\mathrm{D} \cdots \mathrm{A})$ & $220-250$ & $250-320$ & $>320$ \\
$\Phi(\mathrm{DHA})$ & $170-180$ & $>130$ & $>90$ \\
\hline
\end{tabular}

Figure 2.47: Angular scatter plot of $\mathrm{N}-\mathrm{H}^{\cdots} \cdot \mathrm{Cl}^{-}$angles against $\mathrm{H} \cdots \mathrm{Cl}^{-}$distances for $-\mathrm{NH}_{3}{ }^{+}$donors. ${ }^{[19]}$ 


\subsubsection{4,5-Bis(dimethylaminemethylene)acridine (15)}

Major parts of this chapter have already been published in: A. Visscher, S. Bachmann, C. Schnegelsberg, T. Teuteberg, R. A. Mata and D. Stalke, Dalton Trans. 2016, 45, 5689-5699. ${ }^{[11]}$

The fluorescence investigations of 10, 11, and $\mathbf{1 3}$ showed interesting and especially different behaviour, amongst other things, the recognition of analytes and the influence of various solvents. Nevertheless, none of them combined sufficient requirements to be used as a chemical sensor. Equally to H-TrMEDA, another amine which has already been utilised during the master thesis was chosen for further investigation. It features the simplest structure of secondary amines: dimethylamine. In contrast to the $N$-methyl- $N$-phenylamine used in the chapter before, the basicity of dimethylamine is higher due to the twofold $+\mathrm{I}$ effect and the non-existent $+\mathrm{M}$ effect. The fluorescence measurements of the master thesis contained the same systematic error as mentioned in context with 11. Similarly, no solid state structure of the pure compound or of any metal ion complex was determined.<smiles>BrCc1cccc2cc3cccc(CBr)c3nc12</smiles>

8

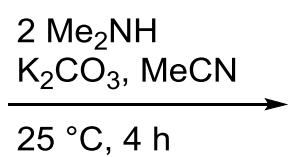

$25{ }^{\circ} \mathrm{C}, 4 \mathrm{~h}$<smiles>CN(C)Cc1cccc2cc3cccc(CN(C)C)c3nc12</smiles>

15

Scheme 2.20: Synthesis of 4,5-bis(dimethylaminemethylene)acridine (15).

The synthesis of $\mathbf{1 5}$ was almost the same as for the other substitution reactions. No heating was needed to obtain the almost pure product after a few hours. The volatile components were removed under reduces pressure and the remaining solids were dissolved in an ethyl acetate/brine mixture. The solution was washed with brine and afterwards dried over $\mathrm{MgSO}_{4}$. After filtration, the solvent was removed under reduced pressure and a partly oily, partly solid product was obtained. Storing the flask in the refrigerator for a couple of hours resulted in a complete yellowbrown solid. A ${ }^{1} \mathrm{H}$ NMR spectrum demonstrated the purity of the target compound without any elaborate purification. Furthermore, the yield was almost tripled (63\%) in comparison to the synthesis in the master thesis (22\%). Since the full spectroscopic characterisation of $\mathbf{1 5}$ confirmed its purity, the luminescence properties of the molecular sensor were investigated in the following chapter.

\subsubsection{Luminescence properties of 4,5-bis(dimethylaminemethylene)acridine (15)}

Likewise to 13, an UV/vis spectrum of $\mathbf{1 5}$ was recorded to evaluate its absorption properties. A suitable excitation wavelength for the emission studies can directly be abstracted by this experiment (Figure 2.48). The absorption spectrum of 15 does not differ significantly to the spectrum of 13. The two highest signals are observed at the same wavelength ( 253 and $356 \mathrm{~nm}$ ), whereby the not determined broad band at $302 \mathrm{~nm}$ was not detectable in this case. This leads to the assumption that it belongs to a transition originated by the phenyl rings of $\mathbf{1 3}$. The signal with its maximum at $356 \mathrm{~nm}$ is a bit more structured in this experiment by what its shoulders can be assigned to the values 340,368 , and $386 \mathrm{~nm}$. The possible observation of this pattern is attributable to resolved vibrational energy levels. 

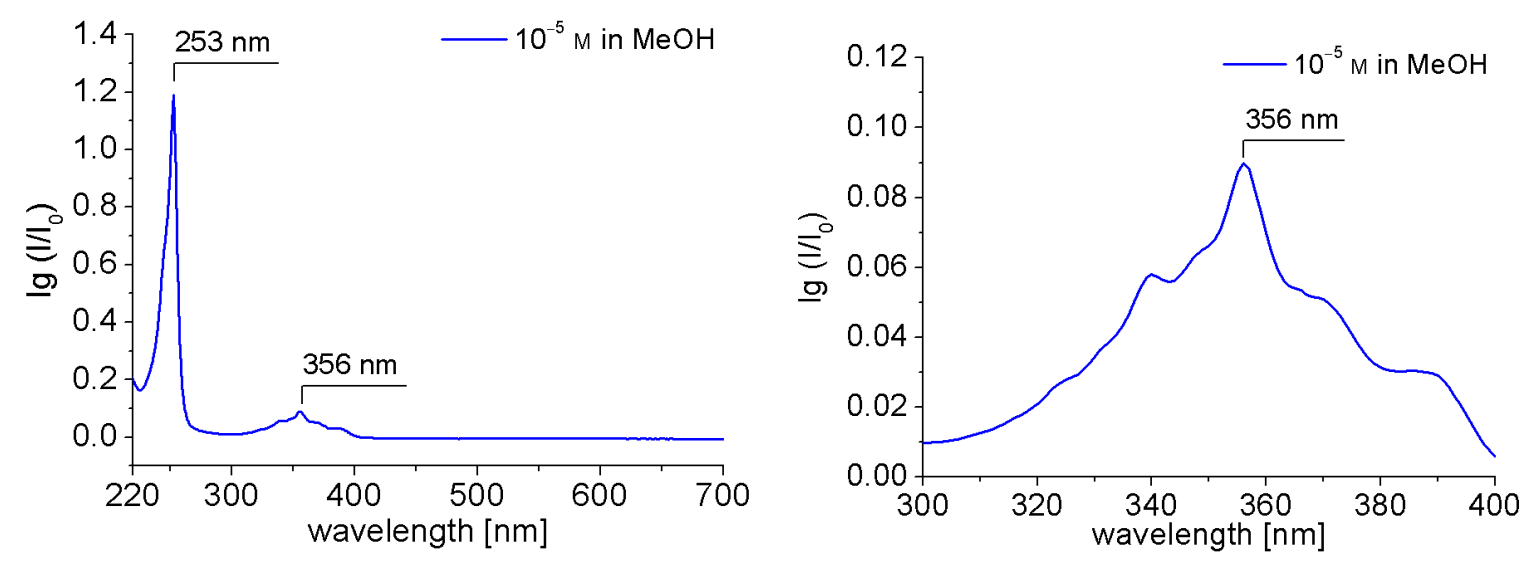

Figure 2.48: UV/vis absorption spectrum of 15 in $\mathrm{MeOH}\left(10^{-5} \mathrm{M}\right)$ showing the complete wavelength range (left) and a detail of the significant wavelength region (right).

As expected, the excitation and emission spectrum in Figure 2.49 are completely different than those obtained during the master thesis with the overall use of $n \mathrm{Bu}_{4} \mathrm{NBF}_{4}$. The resulting bands are higher in intensity in comparison to $\mathbf{1 2}$ and $\mathbf{1 3}$. The emission curve lies in a similar wavelength region with its maximum at $450 \mathrm{~nm}$. Again, the peak at $398 \mathrm{~nm}$ can be assigned to the Raman scattering of methanol.
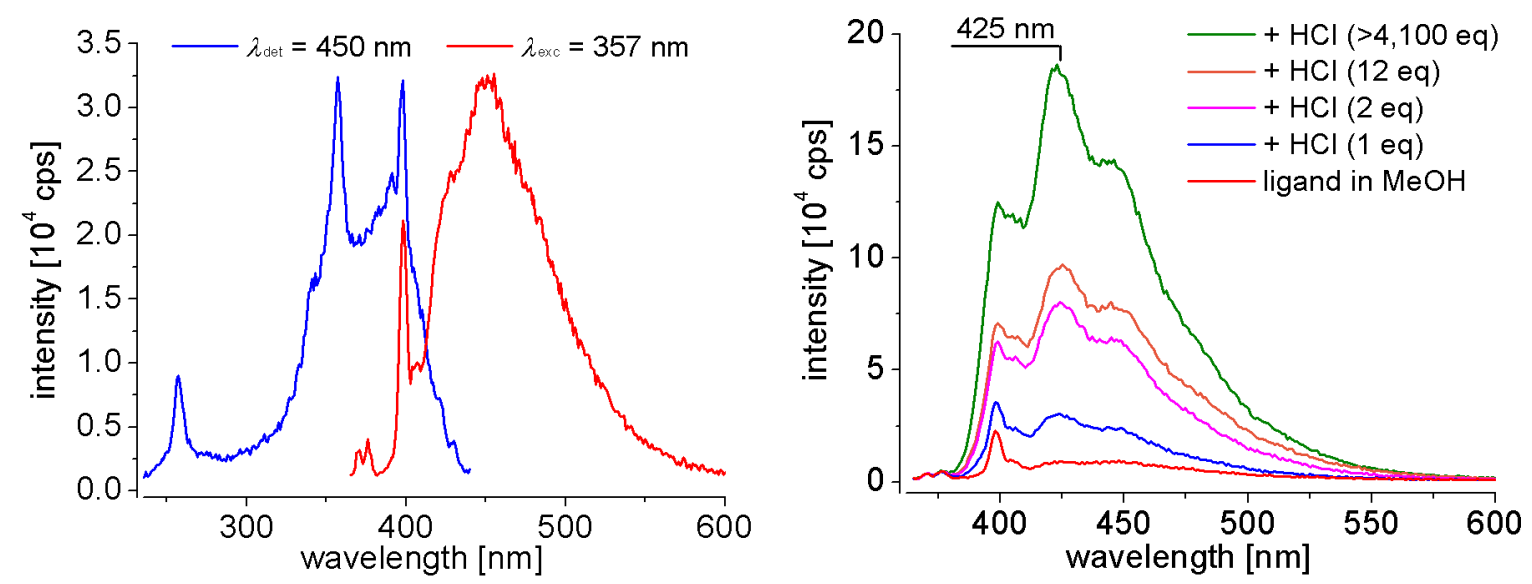

Figure 2.49: Left: Excitation (blue: $\lambda_{\text {det }}=450 \mathrm{~nm}$ ) and emission (red: $\lambda_{\mathrm{exc}}=357 \mathrm{~nm}$ ) spectrum of 15 in $\mathrm{MeOH}\left(10^{-5} \mathrm{M}\right)$; right: emission spectra of $\mathbf{1 5}$ with addition of aqueous $\mathrm{HCl}\left(\lambda_{\mathrm{exc}}=357 \mathrm{~nm}\right)$.

The second highest peak in the excitation spectrum is at a wavelength of $357 \mathrm{~nm}$, which is in line with the results from the absorption study and was used for the excitation of the compound in every following experiment. The protonation of the ligand depicted in Figure 2.49, right, yielded the expected blue shift of the emission band with a maximum of $425 \mathrm{~nm}$. However, the addition of only one equivalent of aqueous hydrochloric acid leads to the hypsochromic shift along with an increase of the fluorescence intensity. Additional amounts of $\mathrm{HCl}$ enhance the intensity until the already known structured curve is formed. In contrast to $\mathbf{1 2}$ and 13, the respond to the acid is considerably more pronounced and already present at fewer equivalents. This observation proves the good donor abilities of the amine receptors according to their high basicity. 


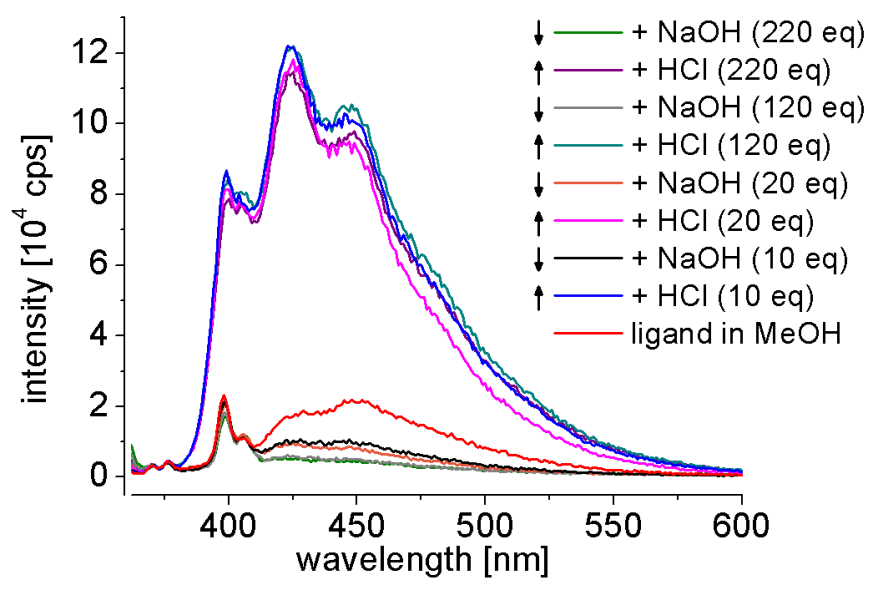

Figure 2.50: Reversibility study with alternating addition of aqueous $\mathrm{HCl}$ and $\mathrm{NaOH}$ to a solution of $\mathbf{1 5}$ in $\mathrm{MeOH}\left(10^{-5} \mathrm{M}, \lambda_{\mathrm{exc}}=357 \mathrm{~nm}\right)$.

As already been shown (Figure 2.24), a reversibility study of the protonation/deprotonation process is useful to determine the stability of the fluorophore. The emission spectra in Figure 2.50 clearly demonstrate the stability of $\mathbf{1 5}$ towards acids and bases. If diluted hydrochloric acid is added to the sample, the emission intensity strongly rises whereas it is effectively quenched by the addition of sodium hydroxide. Each cycle was repeated four times without any alterations. Only the emission curve of the pure ligand in methanol shows a different behaviour. It has a higher intensity than the spectra with an excess of $\mathrm{NaOH}$. The first assumption was the presence of already protonated sensor molecules in the sample. This could be excluded since the wavelength of the starting emission spectrum is above $450 \mathrm{~nm}$. In the case of a protonation of the ligand, the wavelength is shifted to $425 \mathrm{~nm}$. The second possibility would be a small impurity of a metal salt which induces this higher intensity by coordination to the quenching amines. When aqueous $\mathrm{NaOH}$ is added to the solution, possible metal ions should precipitate as hardly soluble metal hydroxides and are no longer available for coordination.

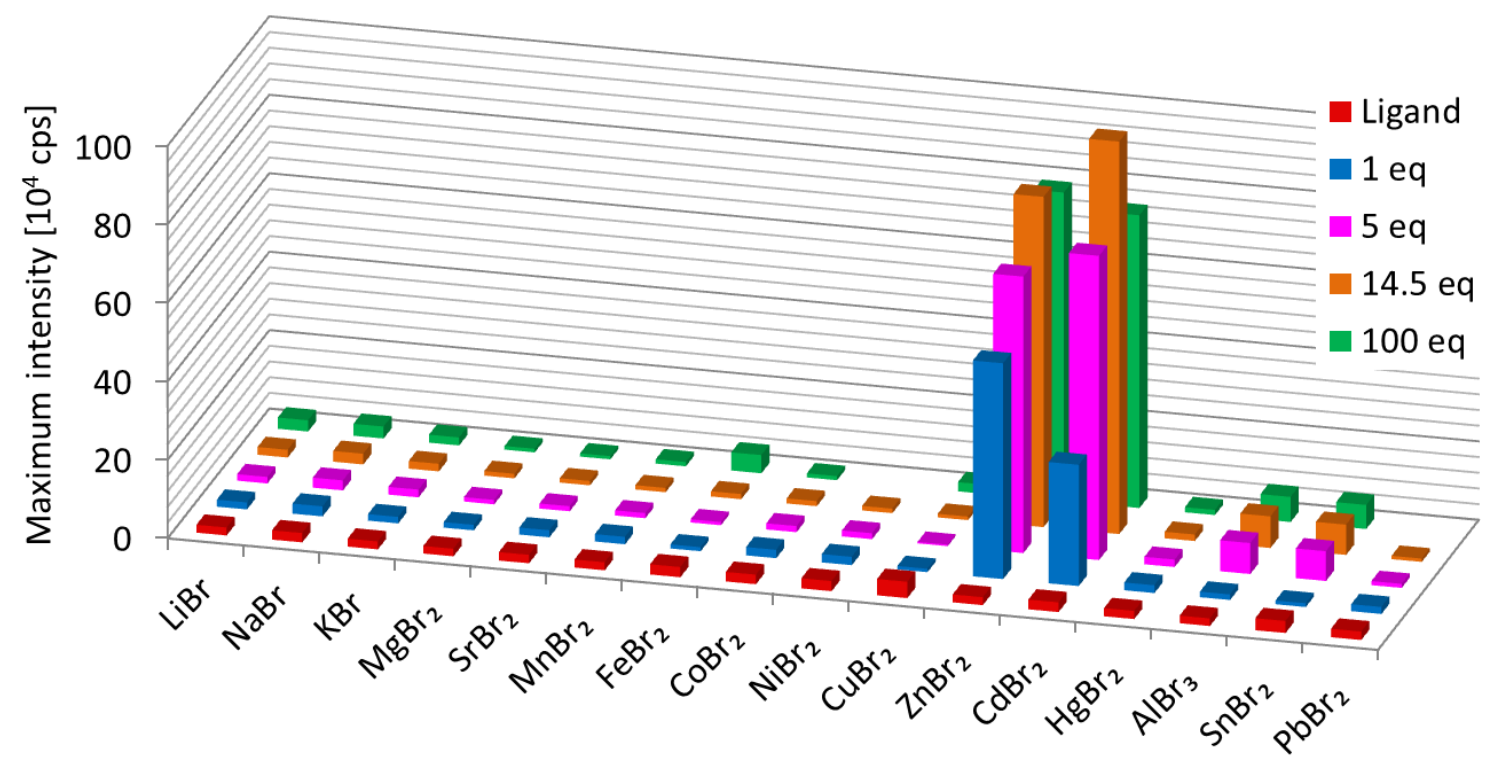

Figure 2.51: Cation screening of 15 in $\mathrm{MeOH}\left(10^{-5} \mathrm{M}, \lambda_{\mathrm{exc}}=357 \mathrm{~nm}\right)$ with different metal salts from 1 to 100 equivalents. The maximum emission intensity is depicted for each metal ion addition. 
The fluorescence response of $\mathbf{1 5}$ towards different metal ions is depicted in Figure 2.51, demonstrating the screening procedure in methanol. Among the tested sixteen metal salts this ligand shows a very high selectivity towards $\mathrm{Zn}^{2+}$ and $\mathrm{Cd}^{2+}$ ions. The emission intensity increases enormously - even higher than in the case of 11. The minute emission enhancement with $\mathrm{AlBr}_{3}$ and $\mathrm{SnBr}_{2}$ is solely due to the strong Lewis acidity of these metal ions which leads to slight protonation of the ligand. This was evidenced by the different resulting emission wavelengths. If the ligand is protonated, the wavelength of the emission maximum is blue-shifted to $425 \mathrm{~nm}$, whereas in the case of $\mathrm{Zn}^{2+}$ or $\mathrm{Cd}^{2+}$ coordination the maximum is observed at $457 \mathrm{~nm}$ and $445 \mathrm{~nm}$, respectively. In this context it should be mentioned that, although the emission shift between the two metal ions is only $12 \mathrm{~nm}$, this is sufficient to differentiate the metals by fluorescence spectroscopy. A closer look at the emission spectra depicted in Figure 2.52 additionally demonstrates the high sensitivity of this sensor molecule.
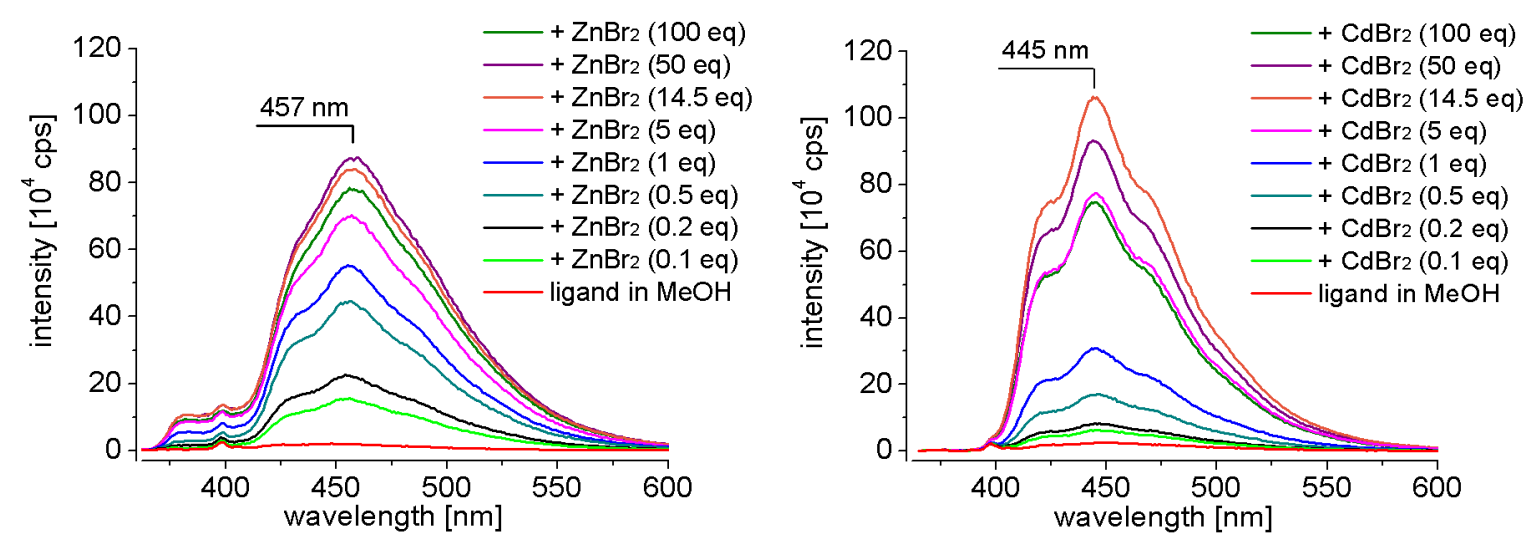

Figure 2.52: Emission spectra of $\mathbf{1 5}$ in $\mathrm{MeOH}\left(10^{-5} \mathrm{M}, \lambda_{\mathrm{exc}}=357 \mathrm{~nm}\right)$ with addition of $\mathrm{ZnBr}_{2}$ (left) and $\mathrm{CdBr}_{2}$ (right), respectively.

After the addition of only 0.1 eq. of $\mathrm{ZnBr}_{2}$, a significant increase of emission intensity was observed. It continued rising until around 50 eq. of the metal ion were titrated into the cuvette. Another 50 eq. resulted in a lowering of the fluorescence signal. At this point all fluorophores are saturated with zinc ions and the dilution effect exceeds. In the case of $\mathrm{CdBr}_{2}$ the altering of the emission spectra is similar to zinc, whereas the fluorescence enhancement rises more slowly and reaches a higher maximum at around 15 equivalents. Since the starting intensities of the pure ligand differ slightly, the emission enhancement is the same for both cases with a factor of around 42.

In Figure 2.53, the UV/vis absorption spectrum of the pure ligand $\mathbf{1 5}$ is compared with the spectra of its metal complexes as well as with the protonated form. In general, the absorption is reduced by the addition of the metal ions whereas it is slightly enhanced through protonation. A shift of the wavelength cannot be observed in any case. In the literature, where similar acridine derivatives were investigated, the altering of the absorption spectra by the addition of metal salts were declared as an evidence for the participation of the acridine nitrogen atom in the complexation with the target metal ion. ${ }^{[57,121]}$ 

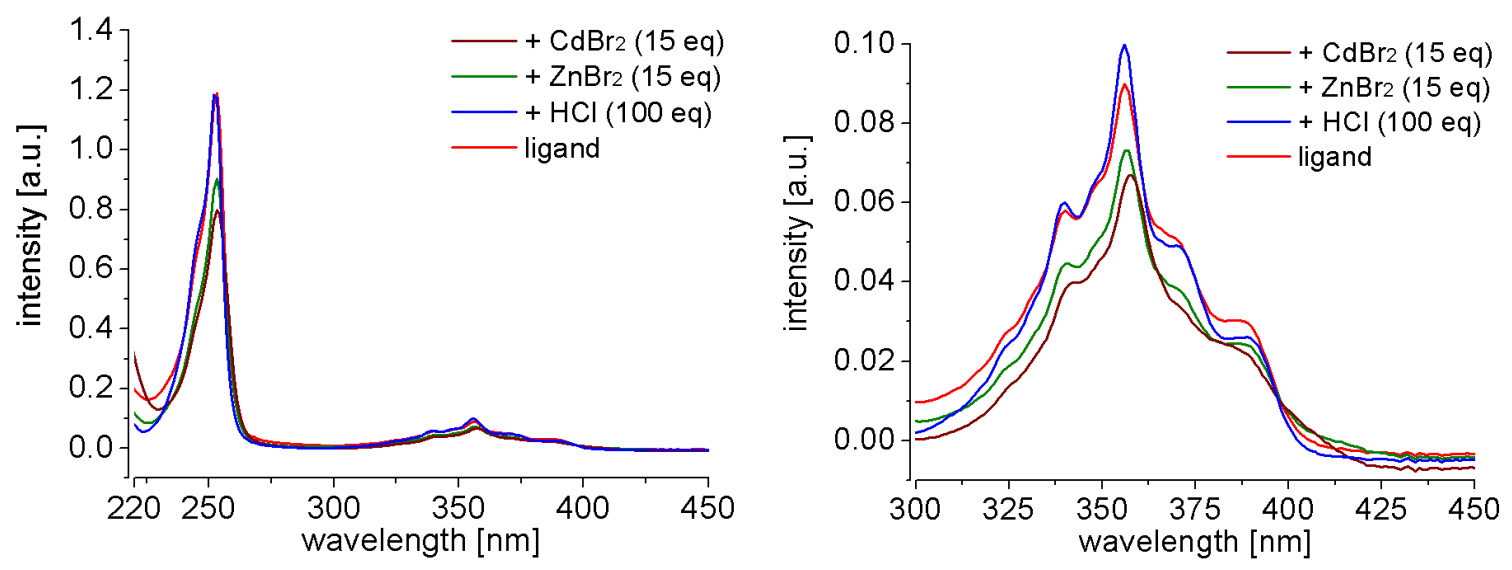

Figure 2.53: UV/vis absorption spectra of four samples: pure $15 \mathrm{in} \mathrm{MeOH}\left(10^{-5} \mathrm{M}\right.$, red), with addition of $100 \mathrm{eq} \mathrm{HCl}$ (blue), with $15 \mathrm{eq} \mathrm{ZnBr}_{2}$ (green), and with $15 \mathrm{eq} \mathrm{CdBr}_{2}$ (brown). In the left figure, the wavelength range from $220-450 \mathrm{~nm}$ is shown whereas in the right figure, a detail of the significant wavelength region around $360 \mathrm{~nm}$ is depicted.

It is stated, that the analyte is affecting the electronic density of the acridine ring and thus affects the electronic transitions. This can be an explanation for the slightly different spectra, but it needs to be considered that the amines of the receptor unit can also influence the aromatic backbone electronically when coordinating to an analyte. Further investigations need to be done before stating possible interactions of the acridine moiety. In this thesis, the coordination of the acridine nitrogen atom to the metal ions is explored in detail in the further chapters.

Regarding the metal ion screening again, the varying starting intensities are the second hint to the existence of contaminations in the sample. The first indication was mentioned during the protonation/deprotonation study. Since the analytics of $\mathbf{1 5}$ do not hint to any significant impurities, the focus was placed on the solvent for the fluorescence experiments. The used methanol was purchased from VWR Chemicals ${ }^{\circledR}$ (AnalaR NORMAPUR) which provides analytical data for their solvents. Each kilogram contains impurities of $<0.2 \mathrm{mg} \mathrm{Zn}^{2+}$ and $<0.01 \mathrm{mg} \mathrm{Cd}^{2+}$ ions. Converted to the employed volume and concentration for the measurements, every sample could contain an amount of $<0.3 \mathrm{eq} \mathrm{Zn}^{2+}$ ions and $<0.009 \mathrm{eq} \mathrm{Cd}^{2+}$ ions with respect to the ligand. This is a significant amount which needs to be considered when comparing the results. In addition, this could explain the higher excitation and emission spectrum of $\mathbf{1 5}$ in comparison to 13, which is not sensitive to the mentioned metal ions. To prove this statement, two further batches of purified methanol were ordered from Sigma-Aldrich ${ }^{\circledR}$ (puriss. p.a.) and Fisher Chemicals ${ }^{\circledR}$ (analytical reagent grade). The batch of the latter company should have less amounts of zinc ions $\left(<0.05 \mathrm{mg}\right.$; $\mathrm{Cd}^{2+}$ was not specified), but the emission spectra measured with this methanol all resulted in higher starting intensities; up to $8.4 \cdot 10^{4} \mathrm{cps}$. Sigma-Aldrich ${ }^{\circledR}$ stated to have less than $<0.1 \mathrm{mg}$ of $\mathrm{Zn}^{2+}$ impurities and $<0.05 \mathrm{mg}$ of $\mathrm{Cd}^{2+}$ in their fillings. The experiments with this methanol gave intensities lower than $1.5 \cdot 10^{4} \mathrm{cps}$ (Figure 2.54). What is more is the difference of the emission wavelength between these batches. The pure ligand seems to emit at smaller wavelengths since the contamination with metal ions (mainly $\mathrm{Zn}^{2+}$ ) shifts the emission maximum to $454 \mathrm{~nm}$ (green and blue line). That is why the excitation and emission spectrum of 15 was recorded again, measured in methanol from Sigma-Aldrich ${ }^{\circledR}$ (Figure 2.54, right). Here, the emission maximum was observed at lower wavelengths at a range of $420 \mathrm{~nm}$ to 
$450 \mathrm{~nm}$. The result of these measurements underlines the sensitivity of the synthesised sensor system which is able to even detect traces of impurities.
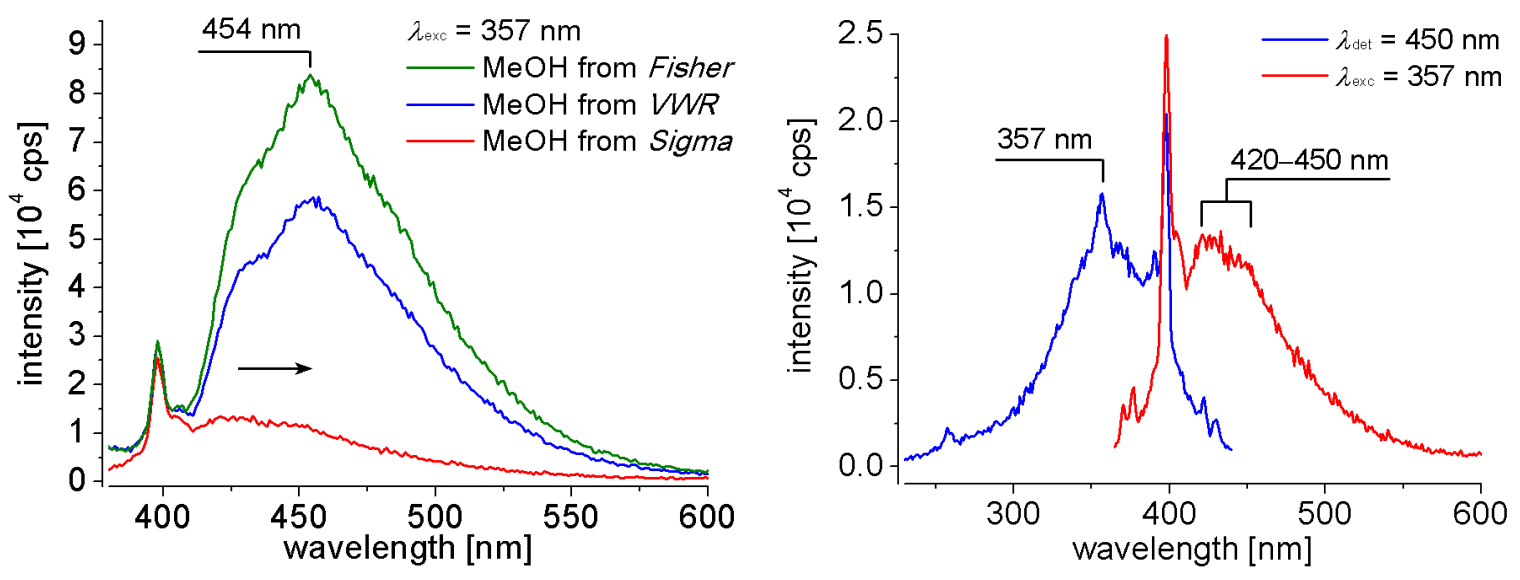

Figure 2.54: Left: Emission spectra of $15\left(10^{-5} \mathrm{M}, \lambda_{\mathrm{exc}}=357 \mathrm{~nm}\right)$ with different batches of methanol from various companies; right: repeated excitation (blue: $\lambda_{\text {det }}=450 \mathrm{~nm}$ ) and emission (red: $\lambda_{\mathrm{exc}}=357 \mathrm{~nm}$ ) spectrum of 15 in MeOH from Sigma-Aldrich ${ }^{\circledast}\left(10^{-5} \mathrm{M}\right)$.

For a good comparability of the resulting spectra, only metal bromides were chosen during the cation screening. Nevertheless, it is interesting to know if, and how other anions would influence the sensing ability of the acridine derivative. For this reason, two more zinc salts were tested under the same conditions: $\mathrm{Zn}\left(\mathrm{NO}_{3}\right)_{2} \cdot 6 \mathrm{H}_{2} \mathrm{O}$ and $\mathrm{Zn}(\mathrm{OAc})_{2} \cdot 2 \mathrm{H}_{2} \mathrm{O}$. Their fluorescence spectra are shown in Figure 2.55.
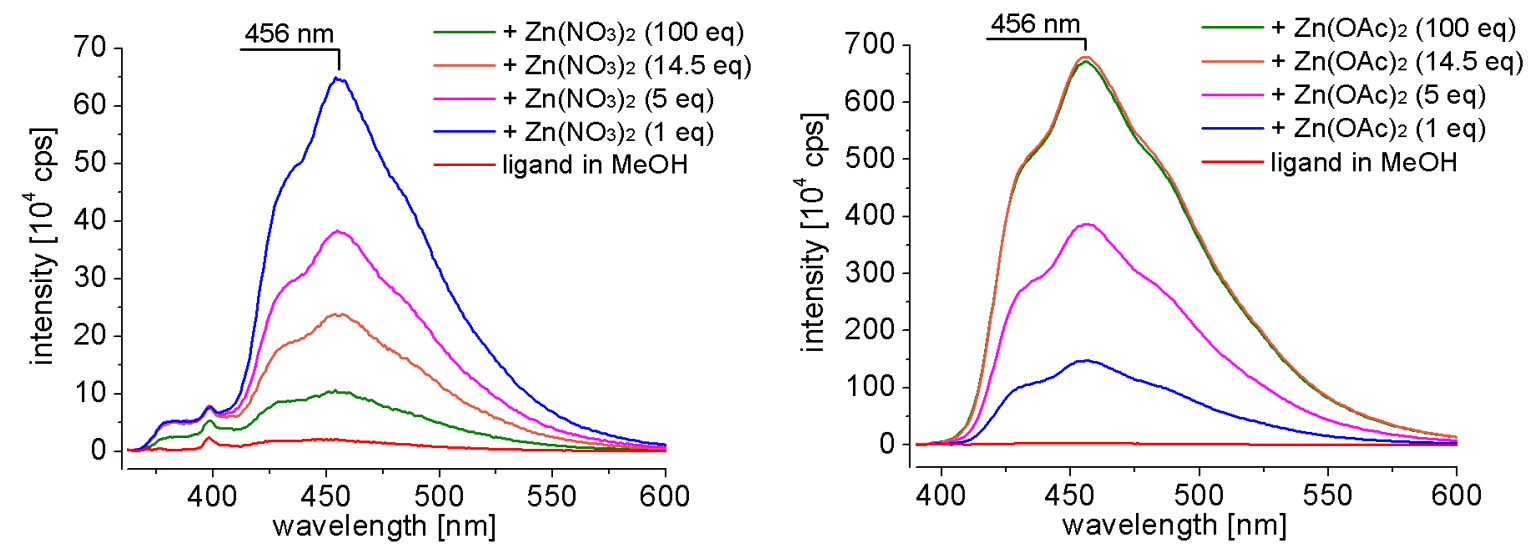

Figure 2.55: Emission spectra of $\mathbf{1 5}$ in $\mathrm{MeOH}\left(10^{-5} \mathrm{M}, \lambda_{\mathrm{exc}}=357 \mathrm{~nm}\right)$ with addition of $\mathrm{Zn}\left(\mathrm{NO}_{3}\right)_{2} \cdot 6 \mathrm{H}_{2} \mathrm{O}$ (left) and $\mathrm{Zn}(\mathrm{OAc})_{2} \cdot 2 \mathrm{H}_{2} \mathrm{O}$ (right), respectively.

On the one hand, the titration experiment with $\mathrm{Zn}\left(\mathrm{NO}_{3}\right)_{2} \cdot 6 \mathrm{H}_{2} \mathrm{O}$ shows a similar behaviour like $\mathrm{ZnBr}_{2}$. The wavelength of the fluorescence band is the same and a strong enhancement of the emission intensity is visible (factor of 30). On the other hand, the maximum is already reached with one equivalent of metal salt added. Further addition directly led to a strong decrease in intensity which cannot be explained only by dilution. In the case of $\mathrm{Zn}(\mathrm{OAc})_{2} \cdot 2 \mathrm{H}_{2} \mathrm{O}$, a tremendous increase of fluorescence is observable which reaches its maximum at around 15 eq. The determined 217-fold increase in intensity is the highest value reported in this thesis. It needs to be mentioned that the used detector in the spectrofluorometer is only able to report intensities up to two million cps reliably. ${ }^{[122]}$ Since the emission intensity lies above this limit, the value of 
the factor contains an unknown error. Nevertheless, the different anions strongly influence the fluorescence output. Since halides are known to be able to quench luminescence, the complexes with metal bromides maybe show lower fluorescence emission, in general, than those without halides. This would explain the much stronger response to zinc with acetate as anions. An explanation for the fast decreasing intensity with an excess of $\mathrm{Zn}\left(\mathrm{NO}_{3}\right)_{2} \cdot 6 \mathrm{H}_{2} \mathrm{O}$ could not been found, yet. The influence of the crystallisation water should not be very significantly. A comparison of the emission spectra of 15 with dry $\mathrm{CdBr}_{2}$ and with its hydrate $\mathrm{CdBr}_{2} \cdot 4 \mathrm{H}_{2} \mathrm{O}$ did not show any difference. What is important to mention is the same shift of the emission wavelength in the cases of all three zinc salts. This supports the hypothesis of the ability to differentiate zinc and cadmium ions by the use of $\mathbf{1 5}$ in combination with a spectrofluorometer.

In Figure 2.50, it was shown that the protonation of the ligand is a reversible process without any significant decomposition. The same can be demonstrated with the reaction of the two coordinating metal ions. Therefore, the bidentate ligand ethylenediamine (en) was chosen to compete with the new sensor compound 15 (Figure 2.56).
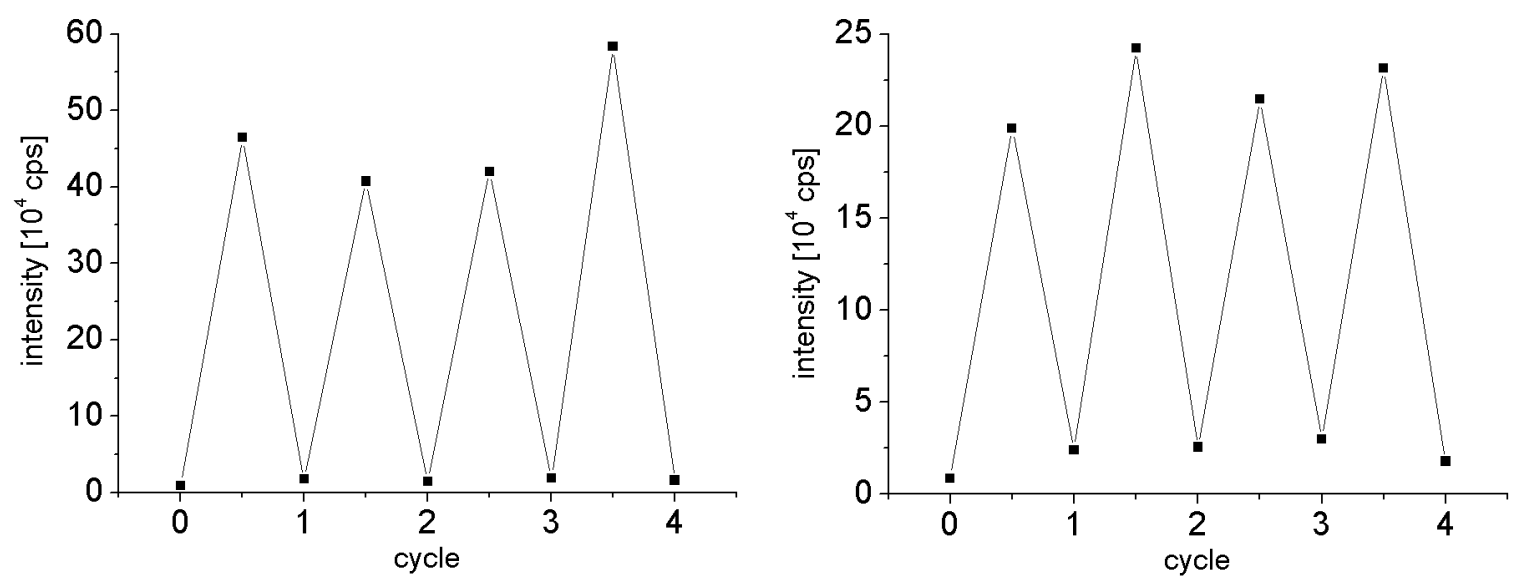

Figure 2.56: Fluorescence intensity changes of $\mathbf{1 5}$ in $\mathrm{MeOH}\left(10^{-5} \mathrm{M}, \lambda_{\mathrm{exc}}=357 \mathrm{~nm}\right)$ upon alternate addition of $\mathrm{ZnBr}_{2}$ (left) respectively $\mathrm{CdBr}_{2}$ (right) and ethylenediamine (en).

In both experiments, the PET effect with its clearly visible off/on switch becomes apparent. Every reagent was added in a slight excess to ensure a full quenching or enhancement of the fluorescence. According to this, the maximum emission intensity varies. This reversibility is a very important feature for a chemical sensor if the compound is used or applied to industrial screening processes. For these purposes, it can be fixed to a polymer or resin surface and only needs to be washed with an e.g. ethylenediamine solution after the usage and thus can be used very often.

The experiments provide further information. Since the addition of five equivalents of ethylenediamine already quenches the fluorescence, the resulting complex must have a higher stability constant than the metal ion complex with 15. Positive charged zinc and cadmium ions form octahedral complexes with three en molecules each $\left(\left[\mathrm{M}(\mathrm{en})_{3}\right]^{2+}\right) \cdot{ }^{[123]}$ The stability constant of these complexes is in both cases $1.23 \cdot 10^{12}$, whereas the successive formation constants do vary.

As already mentioned, zinc is classified as a harder Lewis acid in comparison to cadmium. The nitrogen atoms of the receptor side arms are classified likewise and should therefore bind stronger 
to the zinc ions. This is why an experiment was set up to investigate the behaviour of the competing metal ions (Figure 2.57).
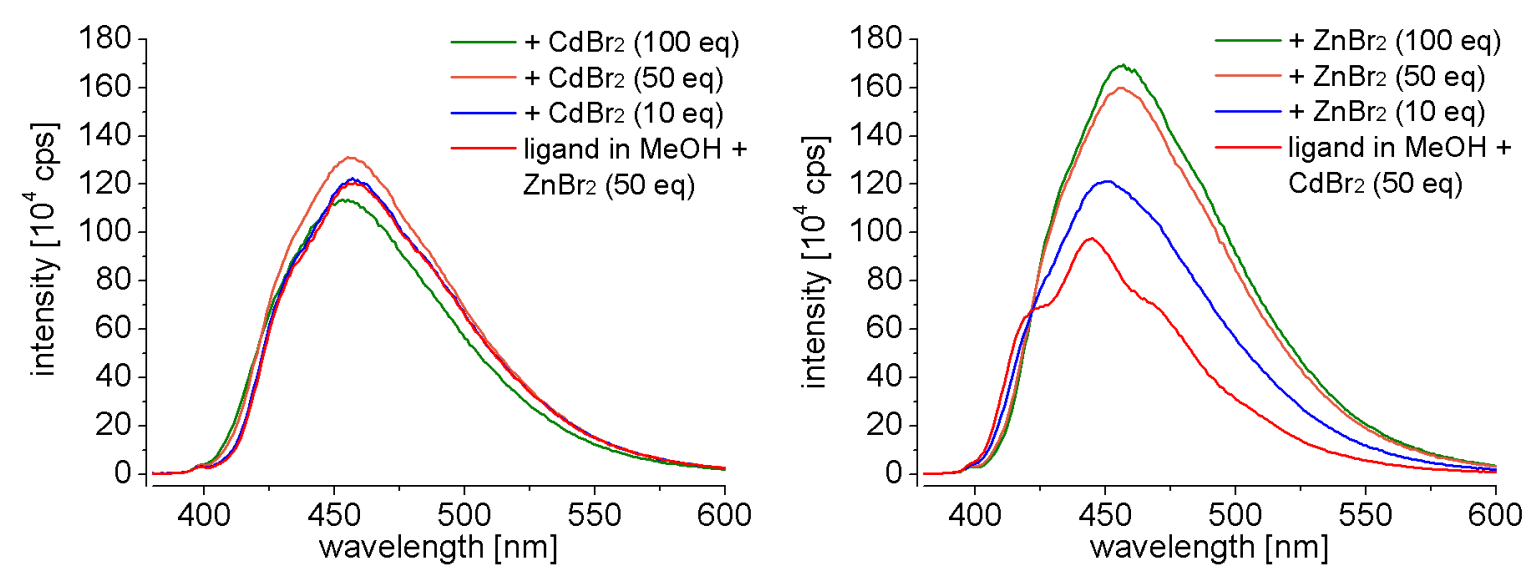

Figure 2.57: Emission spectra in $\mathrm{MeOH}\left(10^{-5} \mathrm{M}, \lambda_{\mathrm{exc}}=357 \mathrm{~nm}\right)$ : First, 15 with 50 eq of $\mathrm{ZnBr}_{2}$ were added, then $\mathrm{CdBr}_{2}$ was titrated into (left) and vice versa (right).

This experiment clearly demonstrates the stronger binding of zinc ions to the sensor ligand in comparison to $\mathrm{Cd}^{2+}$. In the left figure, 50 equivalents of zinc bromide were added to $\mathbf{1 5}$, then cadmium bromide was titrated into with 10,50 , and 100 equivalents. Even with twice of the $\mathrm{Cd}^{2+}$ amount, no significant change of the wavelength or emission intensity was observable. The zinc ions still bind to the amine receptors of the ligand. In the opposite experiment, the results were clearly different (Figure 2.57, right). Already ten equivalents of $\mathrm{Zn}^{2+}$ ions were sufficient to alter the spectrum. In the case of a $1: 1$ ratio of $\mathrm{Zn}^{2+}$ and $\mathrm{Cd}^{2+}$, the spectrum reflects the bonding situation of the zinc complex $\left(\lambda_{\max }=457 \mathrm{~nm}\right)$.

In the introduction, the short lifetime of the fluorescence phenomenon $\left(10^{-6}-10^{-9} \mathrm{~s}\right)$ was discussed. To prove if these values are consistent with the observed emission of the complexes, the lifetime of the excited state of compound $\mathbf{1 7}$ was measured exemplarily. Since this experiment could not be carried out in our department, the sample was taken to a workshop of the company Horiba Scientific in Essen. Here, a single exponential decay with a lifetime of $13.9 \cdot 10^{-9} \mathrm{~s}$ was measured (Figure 7.1) which fits good in the expected region for fluorescent compounds.

All these experiments gave a good overview about the sensing abilities of $\mathbf{1 5}$ in methanol. The next step was, therefore, the investigation of the possibility to use pure water as a solvent. Since the ligand is less polar and does not have any water-soluble substituents like carbon acid or sulfonyl groups, its solubility in water was quite low. For a $10^{-5} \mathrm{M}$ solution only $2 \mathrm{mg}$ of the ligand needs to be dissolved in $682 \mathrm{ml}$ water. However, during the dissolution process the ligand got protonated by the water. The emission spectrum of its solution showed a highly intense band at $425 \mathrm{~nm}$ with exactly the same structure as after protonation with $\mathrm{HCl}$. Even with the use of an aqueous phosphate buffer system $(0.01 \mathrm{M}, \mathrm{pH} 7.4)$, the ligand only went into solution along with its protonation.

Similar observations were made with the addition of water to the alcoholic sample solution (Figure 2.58). Here, the wavelength of the emission maximum is only altered significantly after addition of $1.5 \mathrm{ml}$ water. This is the same volume needed to add 100 eq. of a metal ion. The intensity of the band is, however, not enhanced strongly. 


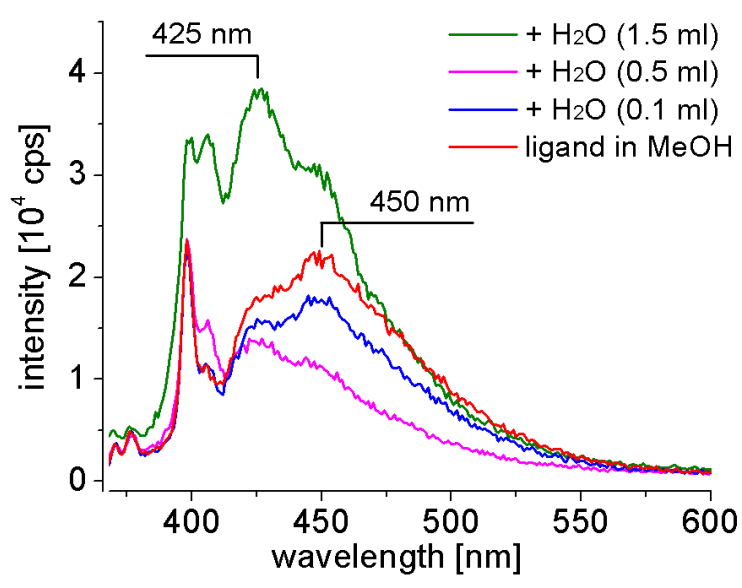

Figure 2.58: Emission spectra of 15 in $\mathrm{MeOH}\left(10^{-5} \mathrm{M}, \lambda_{\mathrm{exc}}=357 \mathrm{~nm}\right)$ with addition of purified water.

This gave rise to the possibility to use water as the solvent for the metal ions, but to stay with methanol for the dilution of the organic compound. By this means, the ligand will not be protonated but can be titrated to aqueous samples in the biological or medicinal analysis. In Figure 2.59, the experiments with $\mathrm{ZnBr}_{2}$ and $\mathrm{CdBr}_{2}$ dissolved in water are illustrated.
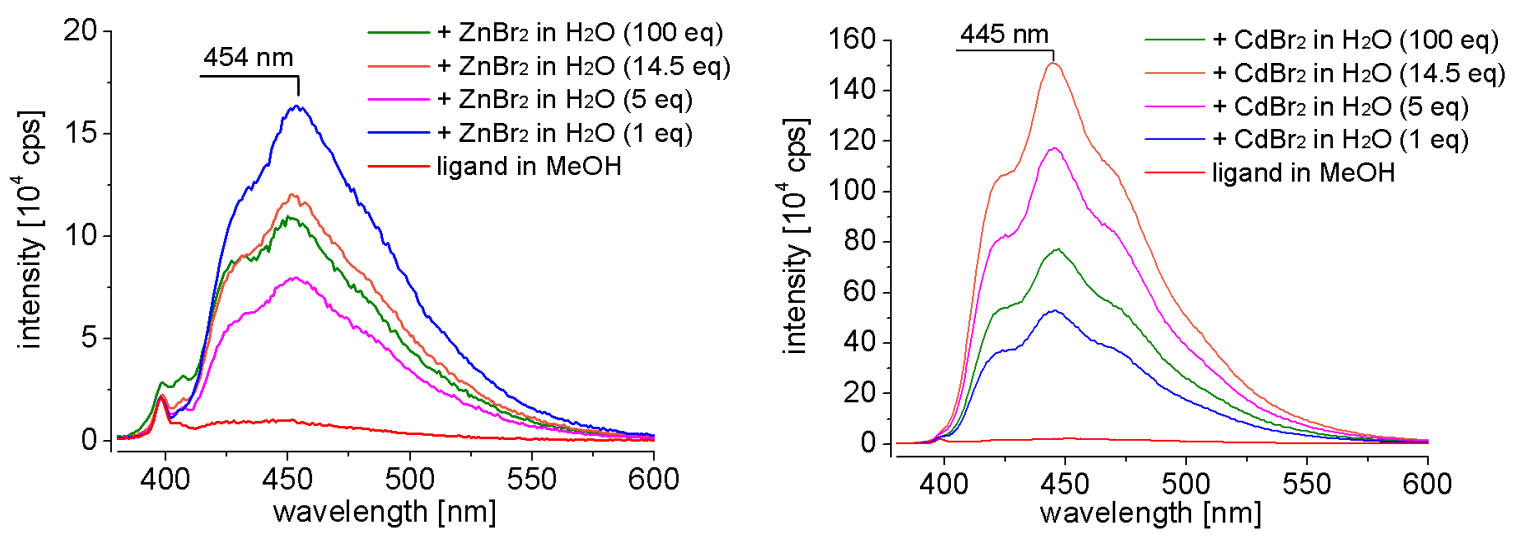

Figure 2.59: Emission spectra of 15 in $\mathrm{MeOH}\left(10^{-5} \mathrm{M}, \lambda_{\mathrm{exc}}=357 \mathrm{~nm}\right)$ with addition of $\mathrm{ZnBr}_{2}$ (left) and $\mathrm{CdBr}_{2}$ (right). Both metal salts were dissolved in purified water.

The addition of one equivalent of zinc ions led to the expected enhancement of the intensity. Unfortunately, five equivalents of the metal ion reduce the emission of light by its half whereas further titration again increases the fluorescence; but only to a smaller extent. This behaviour is hard to explain. In general, the higher donor capability of the $\mathrm{H}_{2} \mathrm{O}$ molecules in comparison to methanol may lead to a lower intensity. The strong solvent shell hardly releases zinc ions for the complexation with 15. To understand the strange leap in the spectrum, the different concentrations of the metal ion solutions need to be considered (Table 2.5). The addition of five equivalents of $\mathrm{Zn}^{2+}$ is accompanied with $0.5 \mathrm{ml}$ of water. This amount already decreases the emission intensity in comparison to the first addition. 14.5 equivalents, however, are added in a higher concentrated solution; only 0.1 millilitres of water are needed for this rise by 9.5 eq. Due to this higher $\mathrm{Zn}^{2+} / \mathrm{H}_{2} \mathrm{O}$ ratio, the fluorescence rises again. Further addition only leads to the often observed effect of dilution.

Surprisingly, this behaviour cannot be observed in the case of cadmium. Here, the addition results in the typical strong increase of the emission intensity. The overall enhancement is even higher as 
with the use of methanol. The different concentrations of the metal solutions did not influence the titration experiment at all. The aqueous solvent shell seems to be less strong in this experiment, which could be attributed to a weaker interaction of the hard Lewis base water with the soft Lewis acid $\mathrm{Cd}^{2+}$ compared to $\mathrm{Zn}^{2+}$. These results demonstrate that sensor compound $\mathbf{1 5}$ dissolved in methanol can be used to detect $\mathrm{Cd}^{2+}$ ions even in aqueous solutions.

For a better assessment of the measured fluorescence values, the emission intensities of several compounds are compared with the typical fluorophore anthracene. In Table 2.9, the maximum intensities of the used compounds and their relative intensities divided by the fluorescence of anthracene are shown. In all cases, the compounds were excited at maximum intensity of the excitation spectrum. The maximum emission intensities of the measured compounds are lower than the intensity of anthracene. Zinc and cadmium bromide in methanol have values close to each other, whereas the addition of $\mathrm{CdBr}_{2}$ dissolved in water reaches a higher relative intensity (0.21). The most powerful enhancement was achieved by the use of $\mathrm{Zn}(\mathrm{OAc})_{2}$ which has almost the same fluorescence intensity as the strong fluorophore anthracene (0.95).

Table 2.9: The maximum emission intensities of the metal complexes of 15 and their relative emission intensities compared with anthracene. All measurements were realised with $10^{-5} \mathrm{M}$ solutions in methanol.

\begin{tabular}{lccc}
\hline \multicolumn{1}{c}{ compound } & $\lambda_{\text {exc }}[\mathrm{nm}]$ & $\begin{array}{c}\text { abs. intensity } \\
{\left[10^{4} \mathrm{cps}\right]}\end{array}$ & $\begin{array}{c}\text { rel. intensity } \\
\text { to anthracene }\end{array}$ \\
\hline \hline $\mathbf{1 5}+\mathrm{ZnBr}_{2}$ & 357 & 84.15 & 0.12 \\
$\mathbf{1 5}+\mathrm{ZnBr}_{2 \text { (aq) }}{ }^{*}$ & 357 & 16.40 & 0.02 \\
$\mathbf{1 5}+\mathrm{CdBr}_{2}$ & 357 & 105.83 & 0.15 \\
$\mathbf{1 5}+\mathrm{CdBr}_{2 \text { (aq) }}{ }^{*}$ & 357 & 151.02 & 0.21 \\
$\mathbf{1 5}+{\mathrm{Zn}\left(\mathrm{NO}_{3}\right)_{2}}$ & 357 & 65.01 & 0.09 \\
$\mathbf{1 5}+\mathrm{Zn}(\mathrm{OAc})_{2}$ & 357 & 678.63 & 0.95 \\
anthracene & 373 & 711.91 & 1.00 \\
\hline
\end{tabular}

* The metal salt was dissolved before in water.

With regard to the results of the metal ion screening in Figure 2.51, it needs to be discussed why the ligand responds selectively towards $\mathrm{Zn}^{2+}$ and $\mathrm{Cd}^{2+}$ ions in alcoholic solution. The effective ionic radii of the targets only play a minor role since the radius of $\mathrm{Zn}^{2+}(74 \mathrm{pm})$ differs stronger to $\mathrm{Cd}^{2+}(95 \mathrm{pm})$ than to most of the other tested cations (e.g. $\left.r\left(\mathrm{Mg}^{2+}\right)=72 \mathrm{pm}, r\left(\mathrm{Cu}^{2+}\right)=73 \mathrm{pm}\right) .{ }^{[24]}$ Furthermore, the receptor side arms can rotate around their methylene bridge to vary the pocket size of the sensor. The electron configuration of the metal ions tends to be more crucial because zinc and cadmium are both $\mathrm{d}^{10}$ elements. In general, the electron configuration of the transition metal ions has a strong influence to the stability constant of possible metal complexes (IrvingWilliams series). ${ }^{[125]}$ However, the heavier homologue $\mathrm{Hg}^{2+}$ did not alter the fluorescence spectrum. This could have other reasons like the presence of fully occupied f-orbitals which leads to a more diffuse electron cloud and a greater ionic radius (116 pm). The radius is $21 \mathrm{pm}$ larger than $\mathrm{Cd}^{2+}$ and could be too big for the chelation by both receptor units. For further investigations, it would be interesting if $\mathrm{Cu}^{+}$and $\mathrm{Ag}^{+}$ions, both $\mathrm{d}^{10}$ metal ions, influence the emission spectrum likewise to $\mathrm{Zn}^{2+}$ and $\mathrm{Cd}^{2+}$. Since their bromide salts are non-soluble in methanol, they have not been tested in the course of this thesis. One example is present in the literature where this hypothesis is not fulfilled. In the introduction, the ligand 4,5-bis $(N, N$-di $(2-$ 
hydroxyethyl)iminomethyl)acridine (BHIA) was discussed as a molecular sensor for $\mathrm{Cd}^{2+}$ ions and, to a smaller extent, for its homologues $\mathrm{Zn}^{2+}$ and $\mathrm{Hg}^{2+}$, too. $\mathrm{Ag}^{+}$ions were also tested but did not influence the present PET effect at all. ${ }^{[57]} \mathrm{A}$ different research group were able to crystallise metal complexes of BHIA with $\mathrm{Cu}^{2+}, \mathrm{Ag}^{+}$, and $\mathrm{Cd}^{2+} \cdot{ }^{[59]}$ All structures demonstrate the interactions of the nitrogen-containing side arms with the metal ions. Accordingly, the PET should be hindered along with the return of fluorescence emission. However, this was only observed by the use of the cadmium salt. $\mathrm{Cu}^{2+}$ ions behave different with regard to their paramagnetism. They are able to quench fluorophores e.g. through electron transfer from the paramagnetic cation to the excited molecule (Figure 2.60, a) or by an energy transfer process via the metal ion (b) ${ }^{[126]}$ This phenomenon was observed by the authors of the fluorescence study. An explanation for the absence of fluorescence by the addition of silver ions to the sensor solution could be found in the experimental conditions of both experiments. The metal ion screening was performed in buffered water whereas the crystals for X-ray diffraction were obtained from an acetone solution. The donor capability of the organic ligand may not be strong enough to compete with the polar aqueous solvent shell.

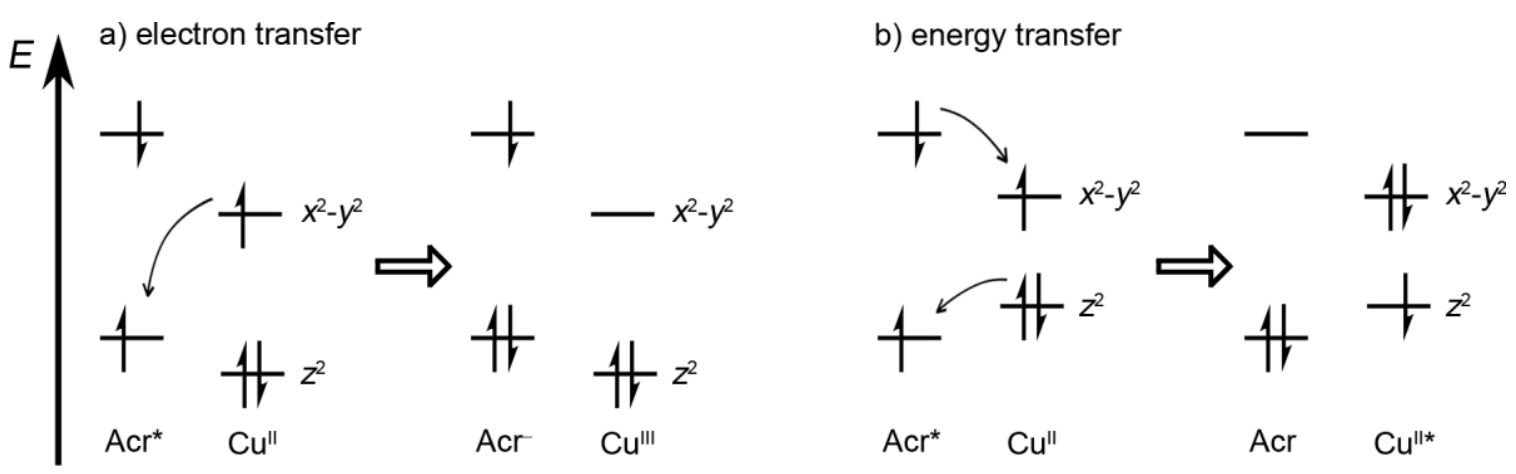

Figure 2.60: Two possible quenching mechanisms of paramagnetic metal ions exemplified by $\mathrm{Cu}^{2+}$ and anthracene. ${ }^{[126 \mathrm{~b}]}$

To conclude, it is possible that some of the tested paramagnetic transition metal ions interact with 15, but do not enhance the emission intensity due to the mentioned quenching mechanisms. Since the ligand is already quenched by the PET of the amine receptor, no significant change was observed upon the titration with the paramagnetic metal ions. Alkaline and earth alkaline metals prefer to bind to oxygen-donor ligands ${ }^{[127]}$ and may stay solvated by the alcoholic environment.

Concerning the interesting studies of the BHIA ligand, crystallographic investigations of several metal ion derivatives of $\mathbf{1 5}$ are done in Chapter 2.3. To begin with, the hydrochloric adduct of the ligand will be discussed in the following.

\subsubsection{4,5-Bis(dimethylaminemethylene)acridine hydrochloride (16)}

Likewise to the synthesis of $\mathbf{1 4}$, hydrochloric acid was used to convert $\mathbf{1 5}$ to its saline derivative (Scheme 2.21). In this reaction, toluene was used as a solvent for $\mathbf{1 5}$ in which conc. $\mathrm{HCl}$ was titrated into. Thereby, the product precipitated and was filtered off. After several washings with toluene, the pure product was dried under reduced pressure and a bright yellow powder was obtained with a good yield of $93 \%$. The compound could be fully characterised by ESI MS, elemental analysis and NMR spectroscopy. With the latter analysis, the protons attached to the nitrogen atoms became apparent and could be identified by their couplings to the neighbouring 
groups. In contrast to the determination of $\mathbf{1 4}$, the elemental analysis gave sufficient results in this case, verifying the existence of two chloride counter ions. Final evidence was given by the solid state structure of the compound.<smiles>CN(C)Cc1cccc2cc3cccc(CN(C)C)c3nc12</smiles>

15

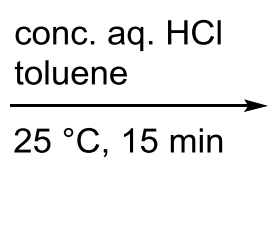

conc. aq. $\mathrm{HCl}$
toluene
$25^{\circ} \mathrm{C}, 15 \mathrm{~min}$

(1)<smiles>CN(C)Cc1cccc2cc3cccc(CN(C)C)c3nc12</smiles>

16

Scheme 2.21: Hydrochlorination of the amine functionalities led to the formation of the saline $\mathbf{1 6}$.
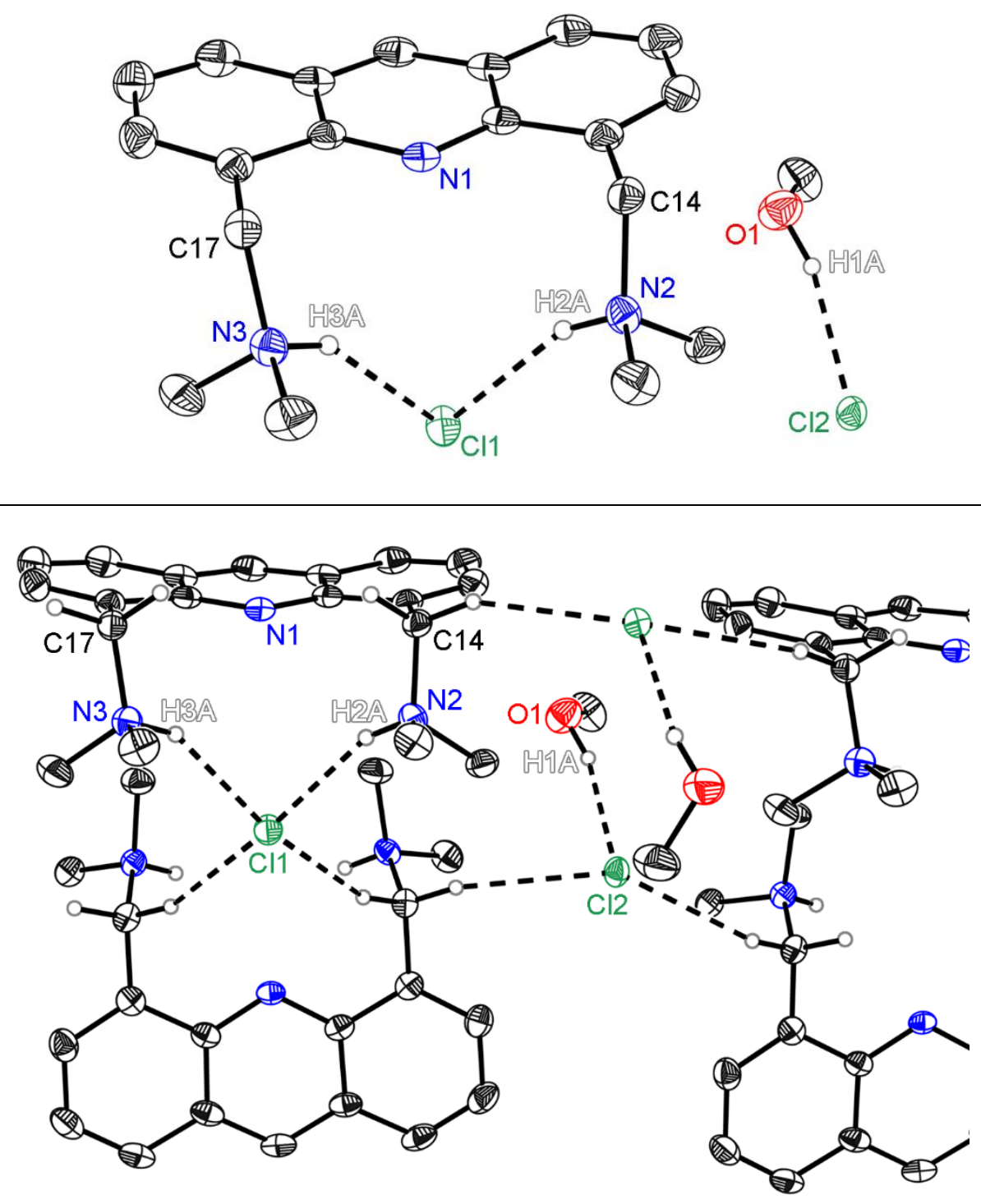

Figure 2.61: Solid state structures of $\left[\left(\mathrm{Me}_{2} \mathrm{NHCH}_{2}\right)_{2} \mathrm{Acr}_{\mathrm{Cl}} \mathrm{Cl}_{2} \cdot(\mathrm{MeOH})(\mathbf{1 6})\right.$ with the asymmetric unit (top) and with an illustration of the hydrogen bonds (bottom). The hydrogen atoms of the acridine and of the methyl groups were omitted for clarity. The positions of the hydrogen atoms H1A, H2A, and H3A were refined freely. Anisotropic displacement parameters are depicted at the 50\% probability level. 
A recrystallisation from methanol gave suitable crystals for X-ray analysis. In Figure 2.61, the solid state structure of $\mathbf{1 6}$ with the co-crystallisation of one methanol is illustrated. The asymmetric unit contains one molecule which crystallises in the orthorhombic space group Pna2 and is shown in the upper part of the figure. The positions of the hydrogen atoms H1A, H2A, and $\mathrm{H} 3 \mathrm{~A}$ were refined freely but $U_{\text {iso }}$ were constrained to $1.2 \cdot U_{\text {eq }}$ of the pivot atom. The second picture demonstrates the linkage of the compounds by hydrogen bonds. One of the chlorine atoms (Cl1) is thereby encaged by two acridine moieties with two stronger $(\mathrm{NH} \cdots \mathrm{Cl})$ and two weaker hydrogen bonds $(\mathrm{CH} \cdots \mathrm{Cl})$. The latter interaction derives from the methylene bridges which also forms hydrogen bonds to the second chloride. This is surrounded by a third hydrogen bond from the methanol molecule $(\mathrm{OH} \cdots \mathrm{Cl})$. Unfortunately, the character of these interactions cannot be determined further. Likewise, the significant bond lengths and angles cannot be discussed since the completeness of the data set is too low (completeness to $\theta_{\text {full }}=77.2 \%$ ). This was caused by a blockage of the cooling system during the measurement along with the loss of the crystal. But in general, the side arms at the 4- and 5-positions are arranged similarly and are comparable with those of their relative precursor 9 .

\subsection{Investigations of metal complexes of $\mathbf{1 5}$}

\subsubsection{4,5-Bis(dimethylaminemethylene)acridine dibromido zinc(II) (17), dibromido cadmium(II) (19), and tetrakis( $\mu$-acetate) bis(acetate) zinc(II) (20)}

To gain a better understanding of the observed fluorescence properties of $\mathbf{1 5}$, it is necessary to know how the metal ions are linked to the ligand. Therefore, the synthesis of the metal complexes and their investigation with a variety of analytical methods is of major importance and is discussed in the following.

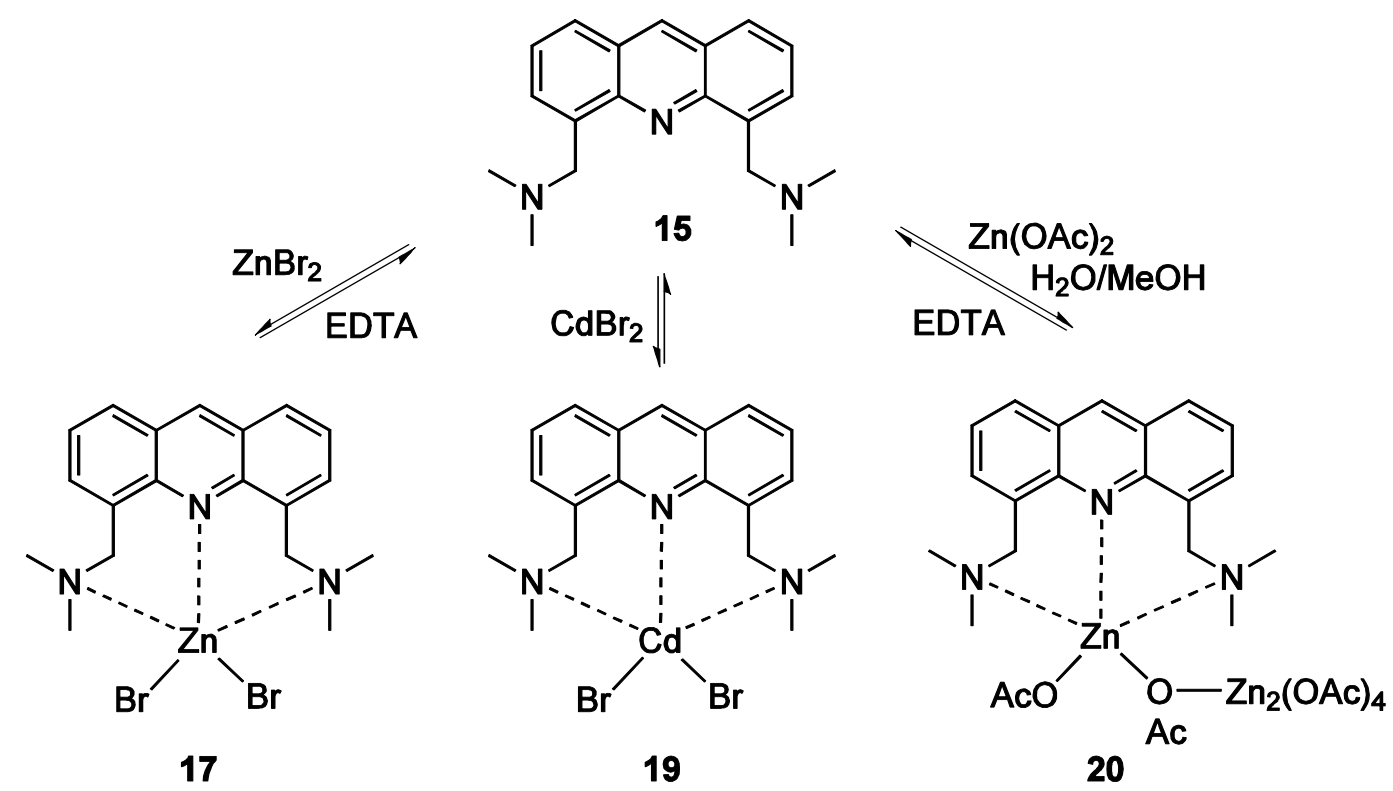

Scheme 2.22: Reaction pathways to different metal complexes based on $\mathbf{1 5}$.

For the synthesis of $\mathbf{1 7}$, the ligand and $\mathrm{ZnBr}_{2}$ were dissolved in THF whereas $\mathrm{MeOH}$ was used for the preparation of 19 (Scheme 2.22). In both cases, one equivalent of the metal salt was added dropwise to 15 , resulting in an immediate precipitation of the metal complex. The suspension was 
filtrated and washed with the same solvent used for the synthesis. The formation of the zinc complex resulted in a moderate yield of $47 \%$. The cadmium complex could be isolated with a yield of $90 \%$. Both zinc complexes were checked for the possibility to reobtain the pure ligand system. Therefore, aqueous $\mathrm{Na}_{2} \mathrm{H}_{2}$ EDTA and $\mathrm{NaHCO}_{3}$ solutions were used to remove the metal salts and to isolate $\mathbf{1 5}$ in good yields (Chapter 4.3).

The crystallisation of $\mathbf{1 5}$ with $\mathrm{Zn}(\mathrm{OAc})_{2}$ to give $\mathbf{2 0}$ was endeavoured to check whether complexes with different zinc salts behave the same which is indeed the case here. The metal salt was only soluble in water and was added in a slight excess to an alcoholic solution of the organic ligand. The yield was not determined for this compound. A toluene/DMF mixture was used to dissolve $\mathbf{2 0}$ at room temperature. The solution was stored at $-30{ }^{\circ} \mathrm{C}$ for ten days resulting in the formation of suitable crystals.

\subsubsection{Solid state analysis by X-ray diffraction}

Single crystals of $\mathbf{1 7}$ and $\mathbf{1 9}$ were obtained through crystallisation from a THF/DMF mixture by slowly cooling the boiling solution to room temperature. By X-ray diffraction experiments, it was possible to determine their solid state structures (Figure 2.62). The zinc complex crystallises in the monoclinic space group $P 2_{1} / c$ and the cadmium derivative in the chiral orthorhombic space group $P 2_{1} 2_{1} 2_{1}$. Both complexes are almost isostructural. In the zinc structure one bromide ion is substituted by a coordinating solvent molecule DMF and is in the following numbered as $\mathbf{1 8}$. The positively charged complex is counterbalanced by a solvent-separated $\left[\mathrm{ZnBr}_{3}\right]^{-}$anion in the asymmetric unit (Figure 5.4).
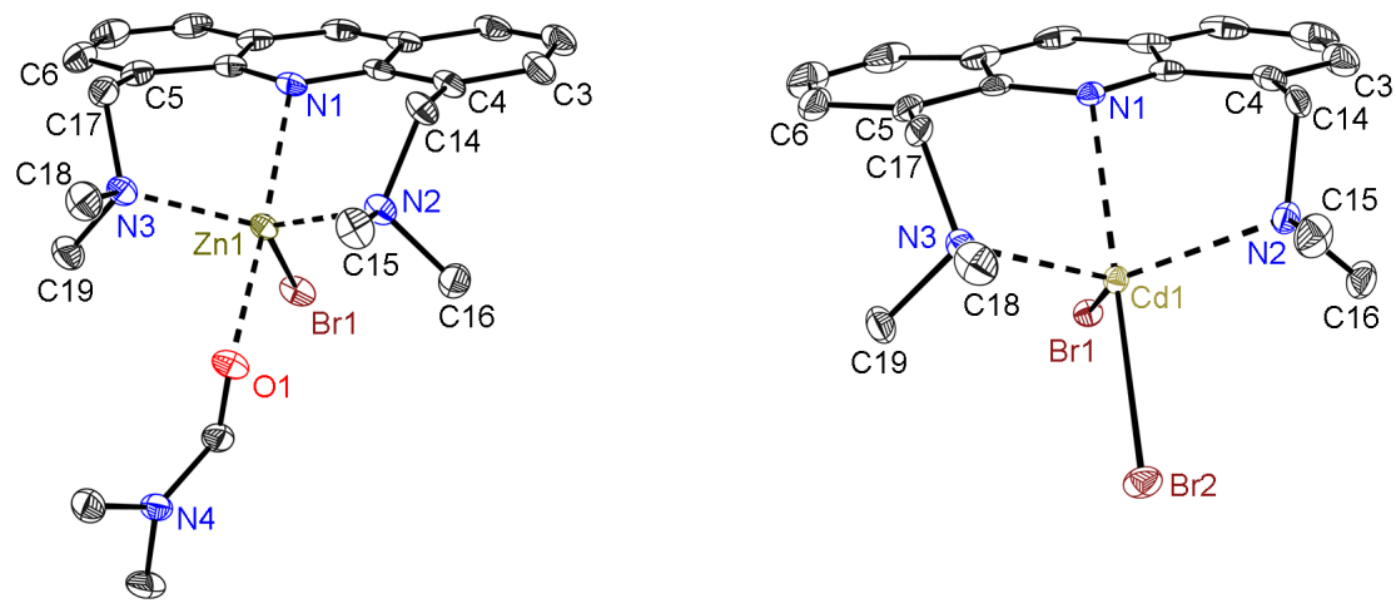

Figure 2.62: Solid state structures of $\left[(\mathrm{dmf}) \mathrm{ZnBr}\left\{\left(\mathrm{Me}_{2} \mathrm{NCH}_{2}\right)_{2} \mathrm{Acr}\right\}\right]^{+} \quad(\mathbf{1 8}, \quad$ left $)$ and $\left[\mathrm{CdBr}_{2}\left\{\left(\mathrm{Me}_{2} \mathrm{NCH}_{2}\right)_{2} \mathrm{Acr}\right\}\right]$ (19, right); crystallised from a THF/DMF mixture. The omitted counter anion in the asymmetric unit of the zinc structure is a solvent-separated $\left[(\mathrm{dmf}) \mathrm{ZnBr}_{3}\right]^{-}$unit. All hydrogen atoms were omitted for clarity. Anisotropic displacement parameters are depicted at the $50 \%$ probability level.

The solid state structures indicate a coordination of the $\mathrm{Zn}^{2+}$ and $\mathrm{Cd}^{2+}$ ions by all three present nitrogen atoms. This binding motif, where the methylene bridged amines in the 4- and 5-positions and the aromatic nitrogen atom of the acridine all are involved in a metal ion coordination, is currently only present with one entry in the CSD. The solid state data was published in 2016 ${ }^{[59]}$ during the writing of this thesis. Other two examples with methylene bridged phosphorous atoms coordinating a ruthenium ion can be found in the database. ${ }^{[128]}$ Overall, a structure search concerning a coordination of an acridine unit to any metal ion merely results in 56 hits. ${ }^{[129]}$ 
Table 2.10: Selected bond lengths and angles of 18 and 19. The angular sums arise from the $\mathrm{C}-\mathrm{N}-\mathrm{C}$ angles.

\begin{tabular}{ccc|ccc}
\hline Bond $[\mathrm{pm}]$ & $\mathbf{1 8}(\mathrm{Zn})$ & $\mathbf{1 9}(\mathrm{Cd})$ & Angle $\left[{ }^{\circ}\right]$ & $\mathbf{1 8}(\mathrm{Zn})$ & $\mathbf{1 9}(\mathrm{Cd})$ \\
\hline \hline $\mathrm{N} 1-\mathrm{M}$ & $230.39(16)$ & $254.07(18)$ & $\mathrm{N} 1-\mathrm{M}-\mathrm{N} 2$ & $90.05(6)$ & $82.91(6)$ \\
$\mathrm{N} 2-\mathrm{M}$ & $207.89(16)$ & $232.31(19)$ & $\mathrm{N} 1-\mathrm{M}-\mathrm{N} 3$ & $89.81(6)$ & $82.81(6)$ \\
$\mathrm{N} 3-\mathrm{M}$ & $209.09(17)$ & $231.70(19)$ & $\mathrm{N} 1-\mathrm{M}-\mathrm{O} 1 / \mathrm{Br} 2$ & $175.31(6)$ & $174.08(4)$ \\
$\mathrm{Br} 1-\mathrm{M}$ & $239.83(4)$ & $259.13(4)$ & $\mathrm{N} 2-\mathrm{M}-\mathrm{N} 3$ & $128.68(6)$ & $120.87(7)$ \\
$\mathrm{O} 1 / \mathrm{Br} 2-\mathrm{M}$ & $218.82(14)$ & $266.16(4)$ & angular sum of N2 & $325.0(5)$ & $327.1(6)$ \\
& & & angular sum of N3 & $324.0(5)$ & $327.0(6)$ \\
& & & $\mathrm{C} 3-\mathrm{C} 4-\mathrm{C} 14-\mathrm{N} 2$ & $-114.8(2)$ & $-105.3(3)$ \\
& & & $\mathrm{C} 6-\mathrm{C} 5-\mathrm{C} 17-\mathrm{N} 3$ & $112.7(2)$ & $105.9(3)$ \\
\hline
\end{tabular}

From the nitrogen-metal bond lengths in Table 2.10 it is obvious that the averaged distances from the amine nitrogen atoms to the metal ion (208.49(17) $\mathrm{pm}$ for $\mathrm{Zn}^{2+}$ and 232.01(19) $\mathrm{pm}$ for $\mathrm{Cd}^{2+}$ ) is shorter than from the acridine nitrogen atom (230.39(16) pm and 254.07(18) pm, respectively). The shorter distances are in perfect agreement with literature values found in the CSD, compared to general $\mathrm{N}-\mathrm{Zn}^{2+} / \mathrm{Cd}^{2+}$ bonds. The longer $\mathrm{N}-\mathrm{M}$ distances can still be found in the database but less frequently. One reason for this different bonding situation results from the rotational ability of the amines to improve the orbital overlap with the target ions. The $\mathrm{C}-\mathrm{N}-\mathrm{C}$ angles of the $\mathrm{sp}^{3}$ hybridised amines are very close to the ideal tetrahedral angle. The resulting geometry forces the metal ion underneath the acridine plane which reduces the dative bonding of the ring nitrogen atom. Moreover, the lone pair of the $\mathrm{sp}^{2}$ hybridised nitrogen atom is partly delocalised in the $\pi$-system of the aromatic system and has therefore a reduced donor capacity. The N-M bond incloses an relatively acute angle of $44.5^{\circ}(\mathbf{1 8})$ and $38.5^{\circ}(\mathbf{1 9})$, to the plane of the central acridine ring. A closer look at the bonding situation and the angle values in Table 2.10 reveals the coordination polyhedron of a trigonal bipyramid at each metal atom. This geometry is depicted in Figure 2.63.
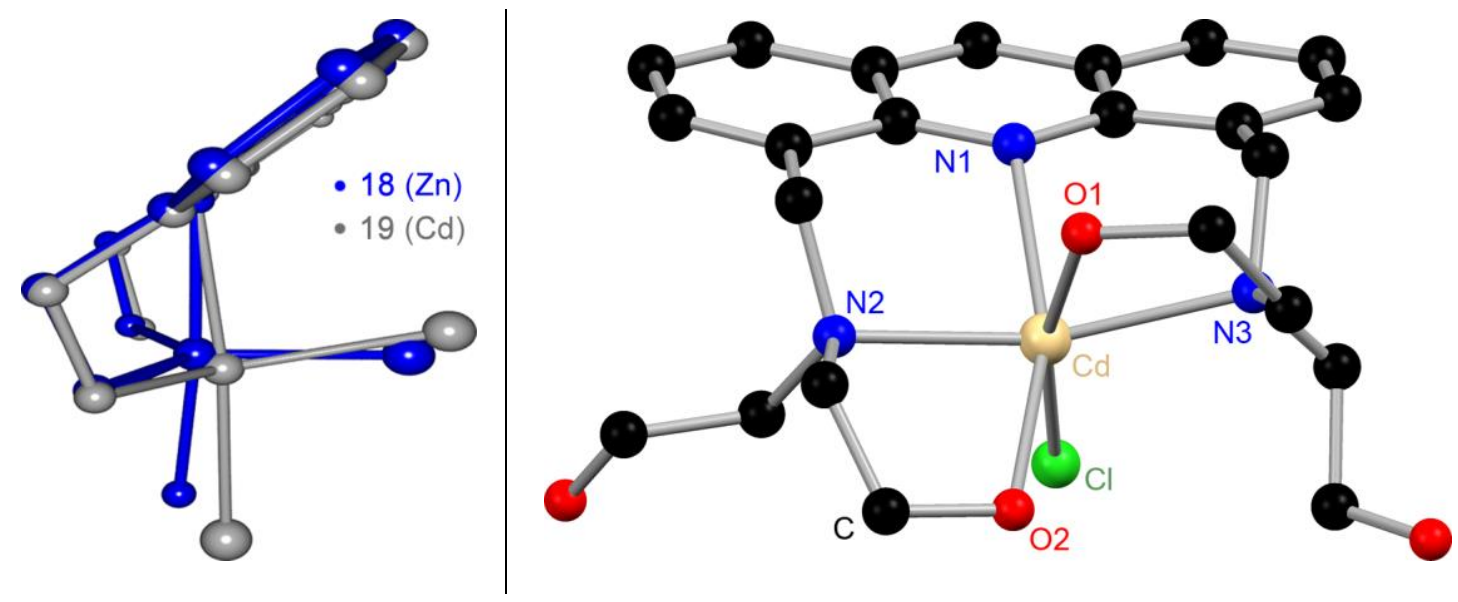

Figure 2.63: Left: Superposition of both metal ion structures (18 and 19), showing the trigonal bipyramidal geometry of the coordination motif. The methyl groups at the amines and the main part of the DMF molecule are omitted for clarity. Right: Solid state structure of [CdCl(BHIA) $]^{+}$, illustrating the similar structure motif of an acridinyl amine sensor system. ${ }^{[59]}$ The solvent-separated counter ion was omitted for clarity. 
The illustration was made by fixing the positions of the inner ring atoms of the acridine backbones. In the zinc complex (blue), the acridine nitrogen atom and the nitrogen atoms of the amines form an almost perfect right angle. In 19 (grey), they are bended to smaller angles due to the higher space requirements of the cadmium ion. Additionally, the torsion angles of the receptor side arms demonstrate this different steric demand. A higher value represents a stronger $\mathrm{N}-\mathrm{M}$ interaction along with a smaller drift of the metal ion underneath the acridine plane (18: $113.8(4)^{\circ}$ vs 19: $105.6(6)^{\circ}$; averaged values). The $\pi$-stacking of the aromatic systems, already discussed in several structures before, can also be observed in the case of $\mathbf{1 8}$. The distance between the two acridine units is $360.1 \mathrm{pm}$, which is the highest value measured in this thesis. The cadmium complex is not showing any interactions between the aromatic backbones.

In Figure 2.63, right, the solid state structure of $[\mathrm{CdCl}(\mathrm{BHIA})]^{+}$is shown, published by Denisova and co-workers. ${ }^{[59]}$ It illustrates the similar structure motif of an acridinyl amine sensor system and its fluorescence properties has been discussed in the introduction (Figure 1.11). In contrast to 19, two more oxygen donors fill the coordination sphere of the metal ion and a chloride acts as counter ion. According to this, the $\mathrm{Cd}^{2+}$ ion has an octahedral coordination geometry which is supported by almost perpendicular angles between the chelating atoms (except O2). The distances of the amines to the metal centre are comparable with those measured for this thesis, whereas the N1-Cd distance is only 246.0(3) pm in comparison to $254.07(18) \mathrm{pm}$ in 19. This stronger interaction may be explained by the five-dentate BHIA ligand inclosing the metal ion to a greater extent. Furthermore, only one chloride ion is bonded, whereas two bromides are present in 19. The anions reduce the positive charge of the cation and makes it less attractive for the lone pairs of the nitrogen atoms.

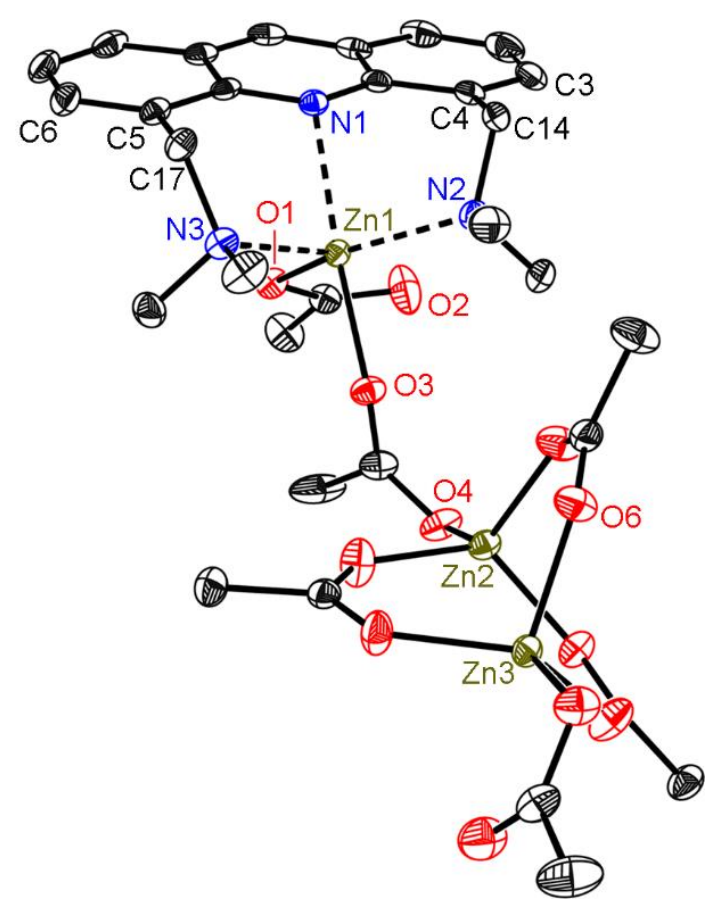

Table 2.11: Selected bond lengths and angles of 20.

\begin{tabular}{cc|cc}
\hline \multicolumn{2}{c|}{ Bond [pm] } & \multicolumn{2}{c}{ Angle [ $\left.{ }^{\circ}\right]$} \\
\hline \hline N1-Zn1 & $232.53(14)$ & N1-M-N2 & $87.88(5)$ \\
N2-Zn1 & $210.35(15)$ & N1-M-N3 & $89.67(5)$ \\
N3-Zn1 & $210.25(15)$ & N1-M-O1 & $85.10(5)$ \\
O1-Zn1 & $197.98(12)$ & N1-M-O3 & $175.85(5)$ \\
O3-Zn1 & $212.50(12)$ & N2-M-N3 & $122.63(6)$ \\
O4-Zn2 & $193.86(13)$ & C3-C4- & $-112.06(19)$ \\
& & C14-N2 & \\
& & C6-C5- & $110.84(18)$ \\
& & C17-N3 & \\
\hline
\end{tabular}

Figure 2.64: Solid state structure of $\left.[\mu \text {-(OAc })_{4}(\mathrm{OAc})_{2} \mathrm{Zn}_{3}\left\{\left(\mathrm{Me}_{2} \mathrm{NCH}_{2}\right)_{2} \mathrm{Acr}\right\}\right] \quad$ (20). All hydrogen atoms were omitted for clarity. Anisotropic displacement parameters are depicted at the $50 \%$ probability level.

In addition to $\mathbf{1 8}$, two more zinc containing structures were crystallised and determined by X-ray diffraction. One of the compounds was crystallised from a THF/MeOH mixture whereby parts of 
the methanol solvent molecules were deprotonated. Two zinc ions are present in the asymmetric unit which are $\mu^{2}$-coordinated by a methoxide anion. The organic ligand is chelating one of the metal ions whereas the second is surrounded by three bromides. One of the bromides is disordered with another methoxide with a site occupation factor of 0.84 and 0.16 , respectively. Additionally, two THF molecules were disordered each over two positions. Since the structure was obtained by chance and the coordination sphere of the metal ions is disordered, it will not be discussed here in detail but can be found in the crystallographic section.

The more interesting structure was obtained by the addition of $\mathrm{Zn}(\mathrm{OAc})_{2}$ to 15 . The metal salt was used to investigate the influence of the counter ion. The difference in their fluorescence behaviour has already been discussed in Chapter 2.2.14, now the properties of the solid state structures will be compared. 20 crystallises in the same monoclinic space group $\left(P 2_{1} / c\right)$ with one molecule in the asymmetric unit (Figure 2.64). The geometry of the zinc chelation is the same as in the case of $\mathbf{1 8 .}$ The only difference is that two anionic acetate groups fill the coordination sphere of the metal ion. One of the acetate groups is bridging to a second zinc ion which is in turn coordinated by another three bridging acetate molecules, linking to a third zinc ion. The $\mathrm{N}-\mathrm{Zn} 1$ distances are elongated by $1.16(32)$ to $2.46(31) \mathrm{pm}$ in the case of $\mathbf{2 0}$ which can be explained by the higher Lewis basic character of the acetate oxygen atoms. ${ }^{[1]}$ Therefore, the positive zinc ion is reduced in its charge and is less attractive for the lone pairs of the nitrogen atoms. This weaker bonding situation can additionally be seen by the smaller torsion angles of the amine groups (C3-C4-C14-N2 and C6C5-C17-N3, respectively).

The N1-Zn1-O3 angle is quite the same as for compound $18\left(\sim 175^{\circ}\right)$. However, the O3-Zn1 bond is with 212.50(12) ppm very high in comparison to the other $\mathrm{Zn}-\mathrm{OAc}$ interactions; they range from $193.86 \mathrm{pm}(\mathrm{O} 4-\mathrm{Zn} 2)$ to $199.92 \mathrm{pm}(\mathrm{O} 6-\mathrm{Zn} 3)$. Since it is an anion-cation interaction, the oxygen-metal bond lengths should be much shorter as in the case of the neutral DMF coordination in 18. Here, the oxygen-zinc distance is only $6.3 \mathrm{pm}$ longer $(218.82 \mathrm{pm})$ than the $\mathrm{O} 3-\mathrm{Zn} 1$ bond of the acetate group. A reasonable explanation for this unusual bond lengths is the shielding of the dimethylamine groups. The mentioned methyl groups would otherwise get in close contact to the methyl groups of the bridging acetate groups of $\mathrm{Zn} 2$ and $\mathrm{Zn} 3$.

\subsubsection{Computational studies on compounds 17-19}

Unfortunately, the results from the solid state investigations do not agree with the observations made during the fluorescence measurements. The nitrogen atom distances (especially N2 and N3) to the central zinc ion should be shorter in the case of 20, since its observed emission intensity was much higher than with $\mathrm{ZnBr}_{2}$. The deactivation of the PET effect of the free ligand $\mathbf{1 5}$ should be more effective when a strong target coordination is present. Apparently, the situation in the solid state cannot be transferred directly to the experiments in solution. Furthermore, the longer $\mathrm{N} 1-\mathrm{M}^{2+}$ distances can still be found in the CSD, however, the solid state investigations are not a sufficient evidence for the existence of a bonding situation. ${ }^{[130]}$ In order to judge on the main binding forces of the metal ion coordination, and to relate the findings to the chemistry in solution, a series of electronic structure calculations on compounds 17 to 19 were conducted.

All structures were optimised at the B3LYP-D3/def2-TZVPP ${ }^{[131]}$ level of theory. Included were the complexes 18 and $\mathbf{1 9}$, identified in the solid state structures, as well as the hypothetical 
complex 17. The structures obtained (see subsequent figures) are in good agreement with the crystal data which has been discussed before. A natural bond orbital (NBO) analysis ${ }^{[132]}$ was carried out to gain insight into the coordination of the metals to the acridine derivative 15 . The data in Table 2.12 give the second order perturbation theory energies ${ }^{[133]}$ for the interaction between the lone pairs of the different nitrogen atoms and the zinc or cadmium ion. Furthermore, the calculated $\mathrm{N}-\mathrm{M}$ distances of the complexes are shown. As expected, in these structures the metal has a weaker coordination to N1, compared to the other nitrogen atoms. ${ }^{[134]}$ Substitution of one bromide anion by a DMF solvent molecule increases this value from 5.6 to $17.0 \mathrm{kcal} \cdot \mathrm{mol}^{-1}$ (17 in comparison to 18). Two different effects could serve as an explanation to this trend. The weaker interaction could be due to a lower positive charge at the metal, since $\mathrm{Br}^{-}$should be a better electron density donor than DMF. Another possibility would be that the bulky bromide anion pulls the metal away from the acridine ring nitrogen atom. The NPA charges for the two compounds are $1.49(\mathbf{1 7})$ and $1.60(\mathbf{1 8})$, so that the charge difference is not very significant. The second possibility, hence the interaction with a second bromide to weaken the coordination to the acridine seems to be the most likely explanation for this effect.

Table 2.12: NBO second-order perturbation theory analysis of the metal ion $(\mathrm{Zn} / \mathrm{Cd})$ coordination to 15 at the B3LYP-D3/def2-TZVPP level of theory.

\begin{tabular}{|c|c|c|c|c|c|c|}
\hline \multirow[b]{2}{*}{ compound } & \multicolumn{3}{|c|}{$E^{(\mathrm{PT} 2)}\left[\mathrm{kcal} \cdot \mathrm{mol}^{-1}\right]$} & \multicolumn{3}{|c|}{$d^{(\mathrm{N}-\mathrm{M})}[\mathrm{pm}]$} \\
\hline & N1 & $\mathrm{N} 2$ & $\mathrm{~N} 3$ & N1 & $\mathrm{N} 2$ & N3 \\
\hline$\left[(\mathrm{dmf}) \mathrm{ZnBr}\left\{\left(\mathrm{Me}_{2} \mathrm{NCH}_{2}\right)_{2} \mathrm{Acr}\right\}\right]^{+}(\mathbf{1 8})$ & 17.0 & 25.4 & 25.1 & 277.0 & 221.3 & 220.1 \\
\hline$\left[\mathrm{ZnBr}_{2}\left\{\left(\mathrm{Me}_{2} \mathrm{NCH}_{2}\right)_{2} \mathrm{Acr}\right\}\right](\mathbf{1 7})$ & 5.6 & 24.7 & 25.4 & --- & --- & --- \\
\hline$\left[\mathrm{CdBr}_{2}\left\{\left(\mathrm{Me}_{2} \mathrm{NCH}_{2}\right)_{2} \mathrm{Acr}\right\}\right]$ (19) & 4.7 & 22.8 & 22.7 & 283.0 & 241.7 & 241.6 \\
\hline $\begin{array}{c}{\left[(\mathrm{MeOH})_{2} \mathrm{Zn}\left\{\left(\mathrm{Me}_{2} \mathrm{NCH}_{2}\right)_{2} \mathrm{Acr}\right\}\right]^{2+}} \\
(\text { in } \mathrm{MeOH})\end{array}$ & 21.9 & 25.4 & 26.5 & 215.7 & 207.0 & 207.0 \\
\hline $\begin{array}{c}{\left[(\mathrm{MeOH})_{2} \mathrm{Cd}\left\{\left(\mathrm{Me}_{2} \mathrm{NCH}_{2}\right)_{2} \mathrm{Acr}\right\}\right]^{2+}} \\
(\text { in } \mathrm{MeOH})\end{array}$ & 15.0 & 32.1 & 31.8 & 237.3 & 225.0 & 225.2 \\
\hline
\end{tabular}

Furthermore, calculations on the hypothetical complexes, $\left[(\mathrm{MeOH})_{2} \mathrm{Zn}\left\{\left(\mathrm{Me}_{2} \mathrm{NCH}_{2}\right)_{2} \mathrm{Acr}\right\}\right]^{2+}$ and $\left[(\mathrm{MeOH})_{2} \mathrm{Cd}\left\{\left(\mathrm{Me}_{2} \mathrm{NCH}_{2}\right)_{2} \mathrm{Acr}\right\}\right]^{2+}$ formed in solution, were conducted. The replication of the conditions in solution as close as possible is of great interest, which is why the COSMO continuum solvation model was applied (for standard methanol solution conditions). ${ }^{[135]}$ The NBO results show that the coordination to the acridine nitrogen is strengthened (21.9 and $15.0 \mathrm{kcal} \cdot \mathrm{mol}^{-1}$ for $\mathrm{Zn}^{2+}$ and $\mathrm{Cd}^{2+}$, respectively). This is in line with the observations made when comparing 17 and 18. The complexes formed in the solid state have a weaker binding to the acridine moiety due to the crystallisation with bromide anions. The computed structures show smaller N1-Zn/Cd distances in agreement with this observation. The values are 216 and $237 \mathrm{pm}$, for $\mathrm{Zn}^{2+}$ and $\mathrm{Cd}^{2+}$ respectively. In methanol solution, sizeable interactions between the metal ion and the N1 nitrogen atom can be confirmed. The interactions with the side arm nitrogen atoms are relatively constant, just slightly enhanced in solution. For the illustration of the difference electron density maps of the discussed compounds, the electron densities of the individual parts were computed and subtracted from the electron density of the whole complex, respectively. Thereby, the geometry of the atoms is untouched. The individual parts are always the organic ligand 15, the metal ion $\left(\mathrm{Zn}^{2+} / \mathrm{Cd}^{2+}\right)$, and the coordinating counterparts $\left(\mathrm{Br}^{-}, \mathrm{DMF}, \mathrm{MeOH}\right)$. This facilitates the 
illustration of the regions where the electron density is enhanced due to the interaction of the relevant atoms. The green clouds represent the accumulated electron density.

In Figure 2.65, the interaction of the lone pairs of all three nitrogen atoms with the zinc ion is accentuated in both structures with a slightly higher accumulated electron density in the methanol compound. This is in line with the results of the computational studies. In the case of the cadmium structures, the interaction with the metal ion is accentuated to a lesser extent. In the compound calculated in solution small electron density clouds are still observable in the region of the nitrogen atoms pointing towards the metal ion centre (Figure 2.66, left). However, the $\mathrm{CdBr}_{2}$ structure does not have any observable interactions, using the same isosurface level. In Figure 2.67, two more electron density maps of $\mathbf{1 9}$ are shown with smaller isosurface levels. With $0.009 \mathrm{e}^{-3}$ the interaction with the side arm amines becomes apparent and at a level of $0.005 \mathrm{e}^{-3}$, the N1-Cd interaction can be observed as well.
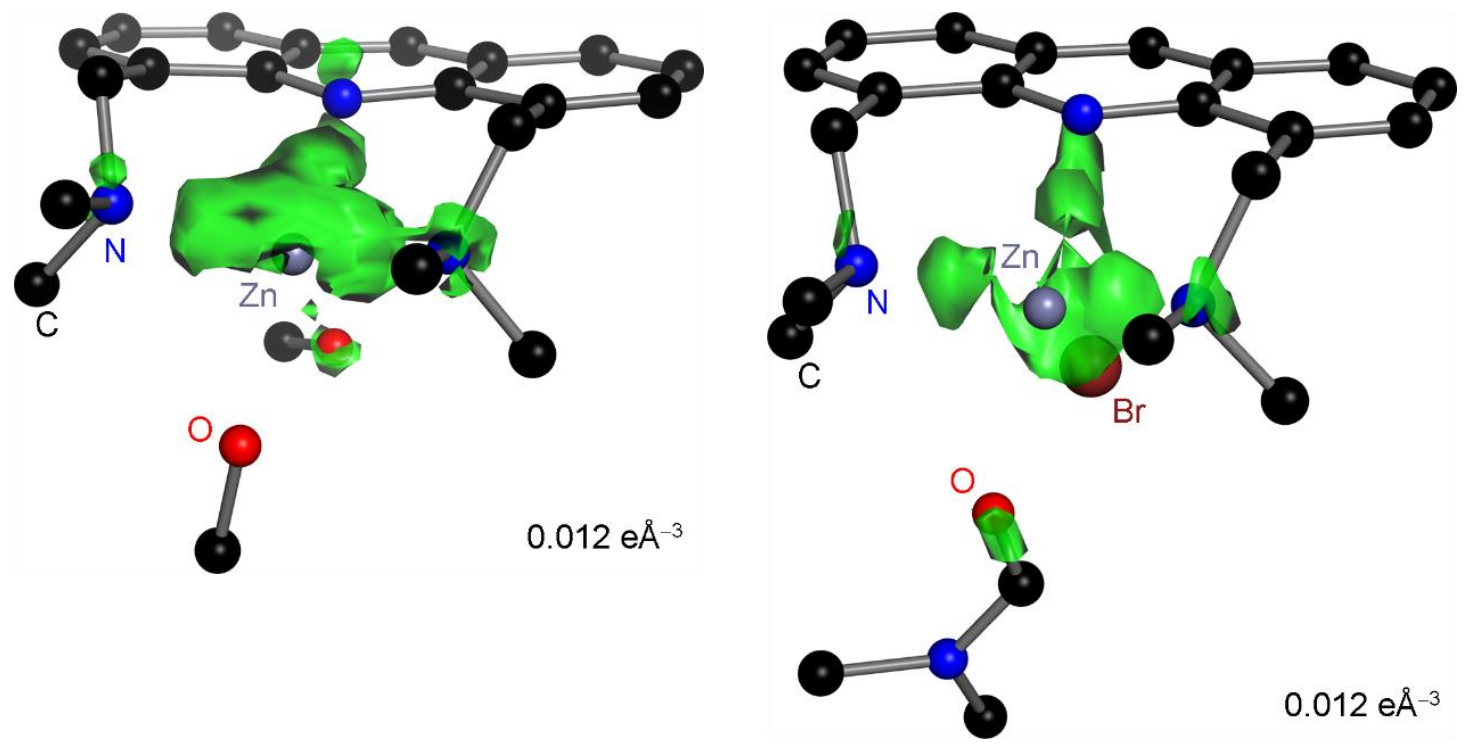

0.012 e $\AA^{-3}$

Figure 2.65: Computed electron density map of $\left[(\mathrm{MeOH})_{2} \mathrm{Zn}\left\{\left(\mathrm{Me}_{2} \mathrm{NCH}_{2}\right)_{2} \mathrm{Acr}\right\}\right]^{2+}$ (left) and $\left[(\mathrm{dmf}) \mathrm{ZnBr}\left\{\left(\mathrm{Me}_{2} \mathrm{NCH}_{2}\right)_{2} \mathrm{Acr}\right\}\right]^{+}(\mathbf{1 8}$, right $)$ at an isosurface level of $0.012 \mathrm{e}^{-3}$, whereof the electron densities of the individual parts have been subtracted.
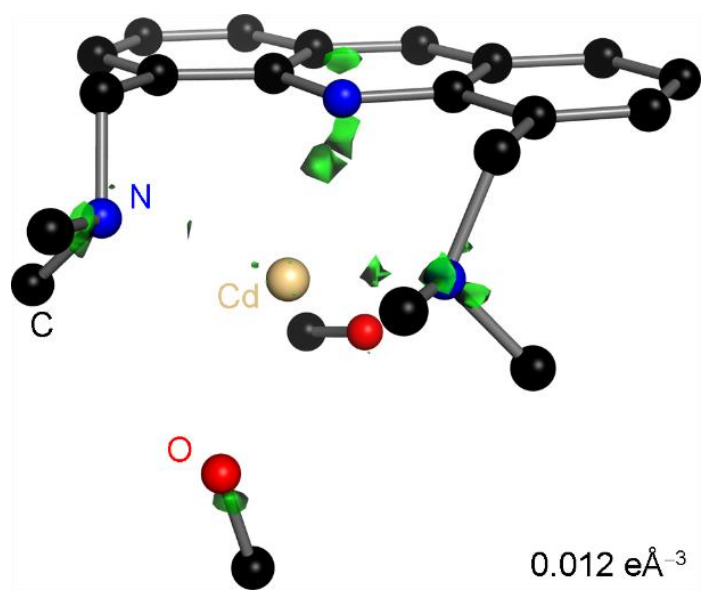

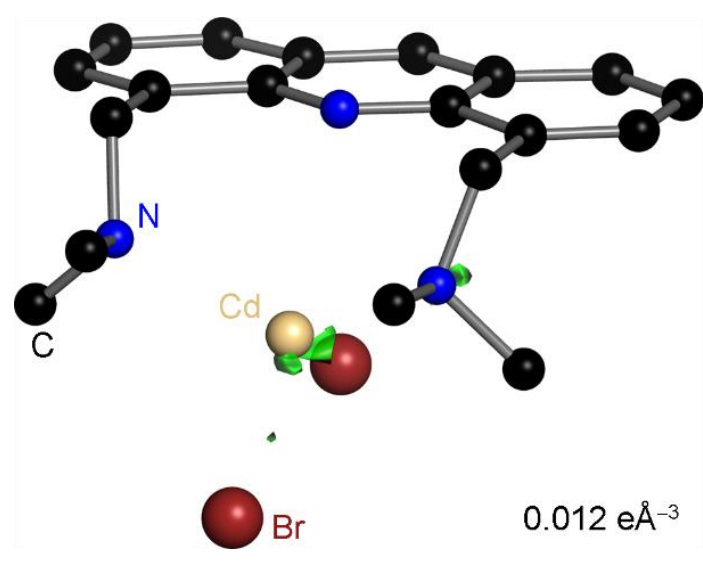

Figure 2.66: Computed electron density map of $\left[(\mathrm{MeOH})_{2} \mathrm{Cd}\left\{\left(\mathrm{Me}_{2} \mathrm{NCH}_{2}\right)_{2} \mathrm{Acr}\right\}\right]^{2+}$ (left) and $\left[\mathrm{CdBr}_{2}\left\{\left(\mathrm{Me}_{2} \mathrm{NCH}_{2}\right)_{2} \mathrm{Acr}\right\}\right](\mathbf{1 9}$, right $)$ at an isosurface level of $0.012 \mathrm{e}^{-3}$, whereof the electron densities of the individual parts have been subtracted. 

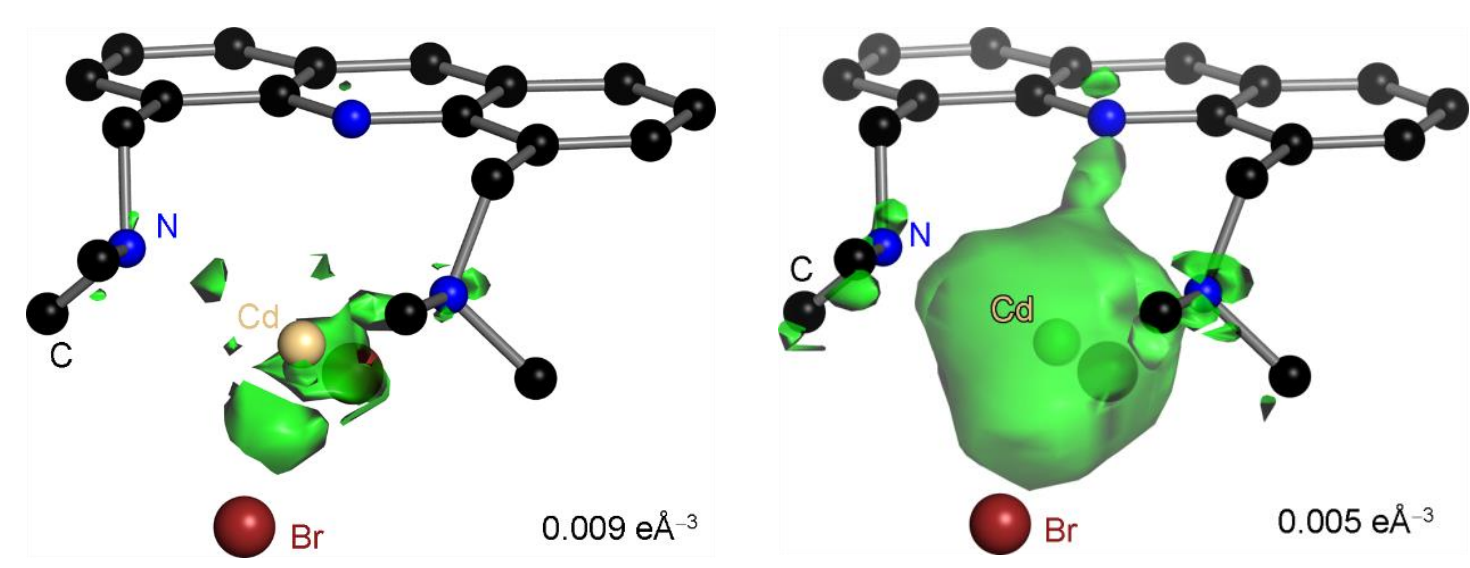

Figure 2.67: Computed electron density map of 19 at different isosurface levels, whereof the electron densities of the individual parts have been subtracted. Left: $0.009 \mathrm{e} \AA^{-3}$, right: $0.005 \mathrm{e}^{-3}$.

When comparing the results of the computational studies with the fluorescence properties, it is apparent from the difference density plots that the metal coordination does not affect the $\pi$-density significantly in agreement with the almost unchanged emission wavelength. A possible explanation would be that the coordination does not influence the $\pi$-system significantly. Considering the geometry optimisations for the complex in methanol solution, both metal ion interactions with the aromatic nitrogen atom become stronger. This should also be the case for the titration experiment of the fluorescence measurements.

\subsubsection{Investigation of the metal complexes in solution}

For a deeper insight into the liquid phase, ${ }^{1} \mathrm{H}$ NMR spectra in DMSO- $d_{6}$ were recorded of the relevant compounds depicted in Figure 2.68. In comparison to the spectrum of the pure ligand, the metal complexes show significantly broadened signals. This is often caused by dynamic processes. ${ }^{[18]}$ Consequently the spectra of $\mathbf{1 7}$ and $\mathbf{1 9}$ were measured at different temperatures.

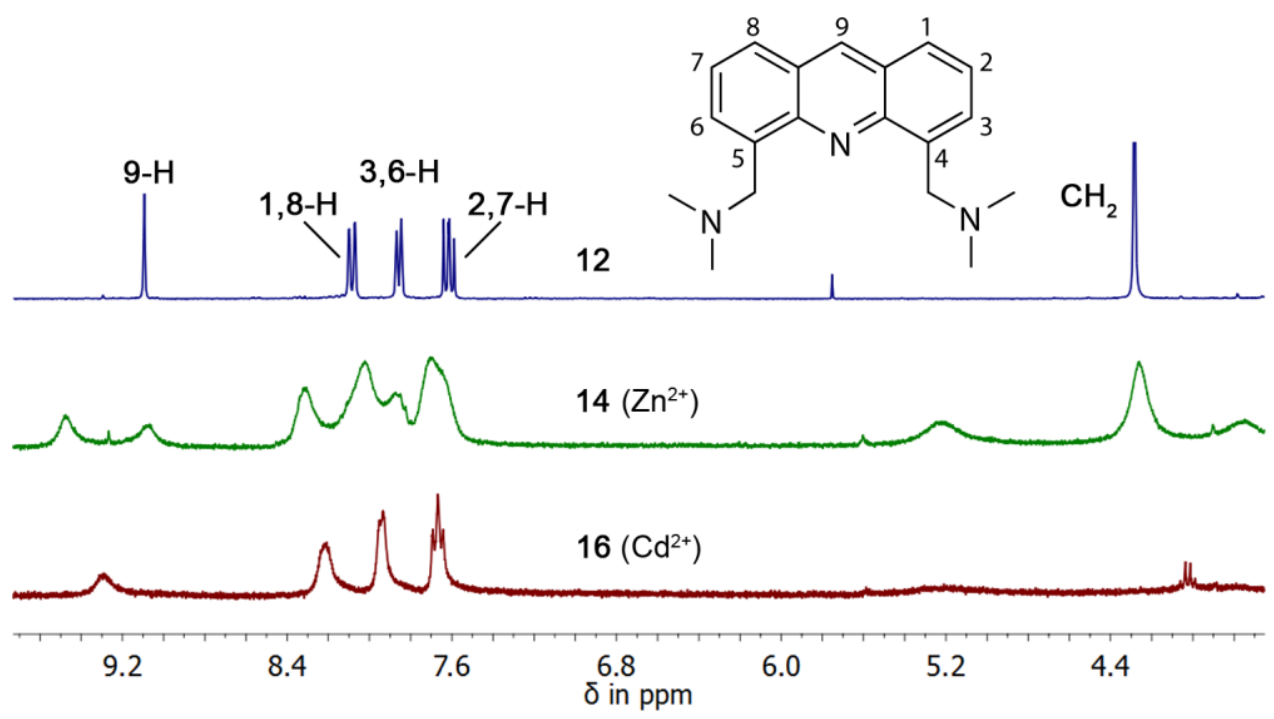

Figure 2.68: ${ }^{1} \mathrm{H}$ NMR spectra of compounds 15, 17, and 19 in DMSO- $d_{6}$ at ambient temperature.

Therefore, the NMR solvent was changed to DMF- $d_{7}$ due to its wide temperature range of liquidity from -61 to $151{ }^{\circ} \mathrm{C}$. Another advantage is the higher comparability to the solid state structures since they were crystallised from the same solvent. Especially the zinc structure 
contains a DMF molecule coordinating to the metal ion. This motif will be discussed in this chapter, too. With the use of DMF- $d_{7}$ for the NMR experiments, the signals are likewise broadened at ambient temperature. An increase of the temperature sharpens the broad signals until one definite set of signals is obtained (Figure 2.69, 353 K). At the coalescence temperature of nearly $303 \mathrm{~K}$, the diastereotopicity of the $\mathrm{CH}_{2}$ protons becomes visible. The signal of the four methyl groups at the amines also splits into two signals.

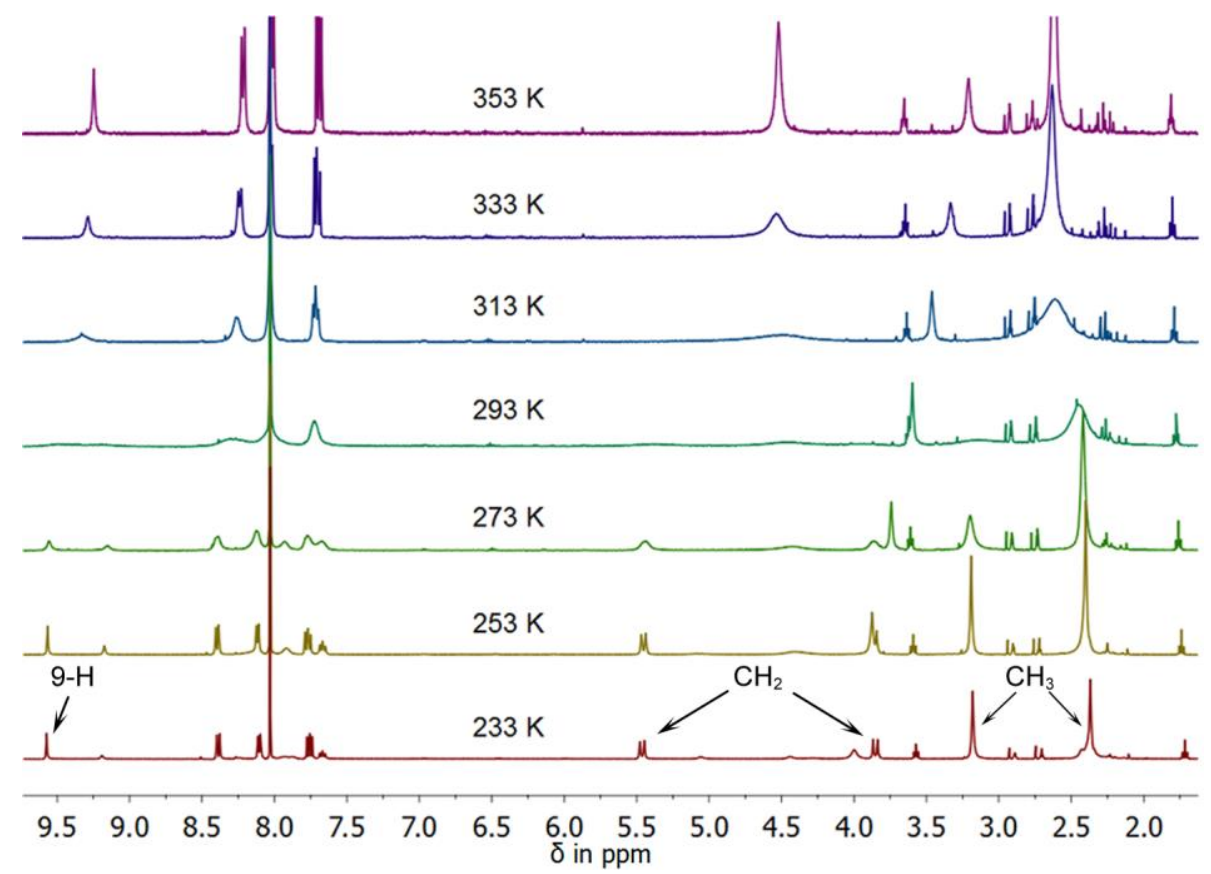

Figure 2.69: Temperature-dependent ${ }^{1} \mathrm{H}$ NMR spectra of $\mathbf{1 7}$ in DMF- $d_{7}$. The discussed signals are marked at $233 \mathrm{~K}$.<smiles>CN(C)Cc1cccc2cc3cccc(CN(C)C)c3nc12</smiles>

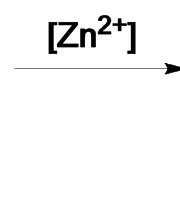

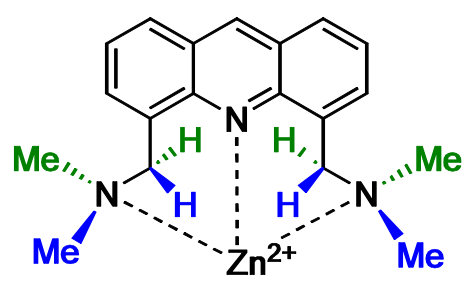

Scheme 2.23: Visualisation of the hindered rotation of the amines after the coordination of zinc. The coloured protons and methyl groups are no longer chemically equivalent and can be monitored.

The velocity of their hindered rotation caused by the coordination of the zinc ion (Scheme 2.23) lies underneath the NMR timescale at lower temperatures and can be monitored. At lower temperatures, the spectrum splits into two different sets of signals with a maximum intensity at around $253 \mathrm{~K}$. At this temperature they exhibit an intensity ratio of around 2:1 what can be monitored by the singlet of the H-9 proton at $9.57 \mathrm{ppm}$ and $9.17 \mathrm{ppm}$, respectively (Figure 2.70). This splitting is most likely to be attributed to the exchange of the bromine atoms with the solvent DMF, which has already been observed in the solid state structure. To prove this statement, an excess of $\mathrm{NaBr}$ was added to the sample, altering the ${ }^{1} \mathrm{H}$ NMR spectrum significantly: a third set of signals appears whereby the intensity ratio of the other two sets of signals is reversed (Figure 2.71). To distinguish between these different species, two pseudo $2 \mathrm{D}{ }^{1} \mathrm{H}$-DOSY experiments at 
low temperature were carried out, with and without the presence of $\mathrm{NaBr}$ salt (Figure 2.72; for full spectrum see Figure 7.3).

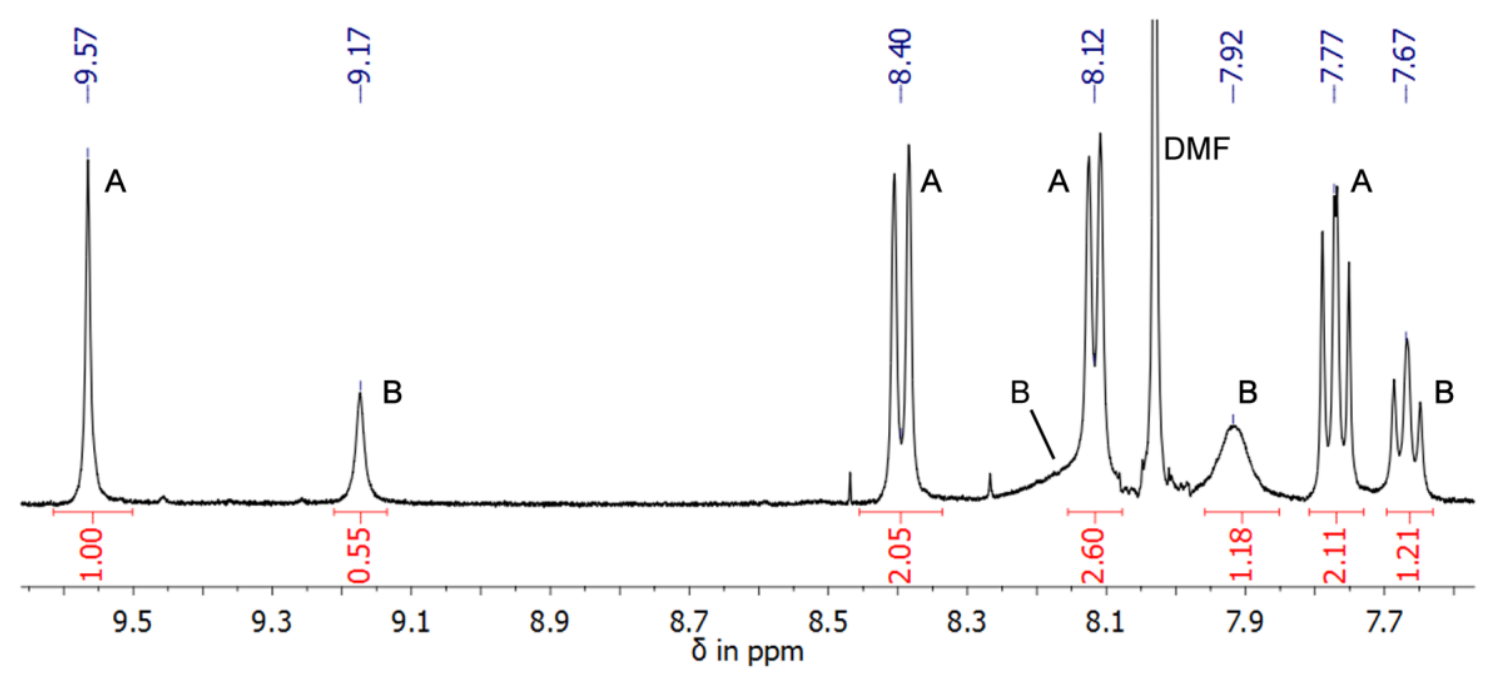

Figure 2.70: Aromatic region of the ${ }^{1} \mathrm{H}$ NMR spectrum of $\mathbf{1 7}$ in DMF- $d_{7}$ at $253 \mathrm{~K}$, illustrating the two sets of signals ( $\mathbf{A}$ and $\mathbf{B}$ ) observed at lower temperatures.

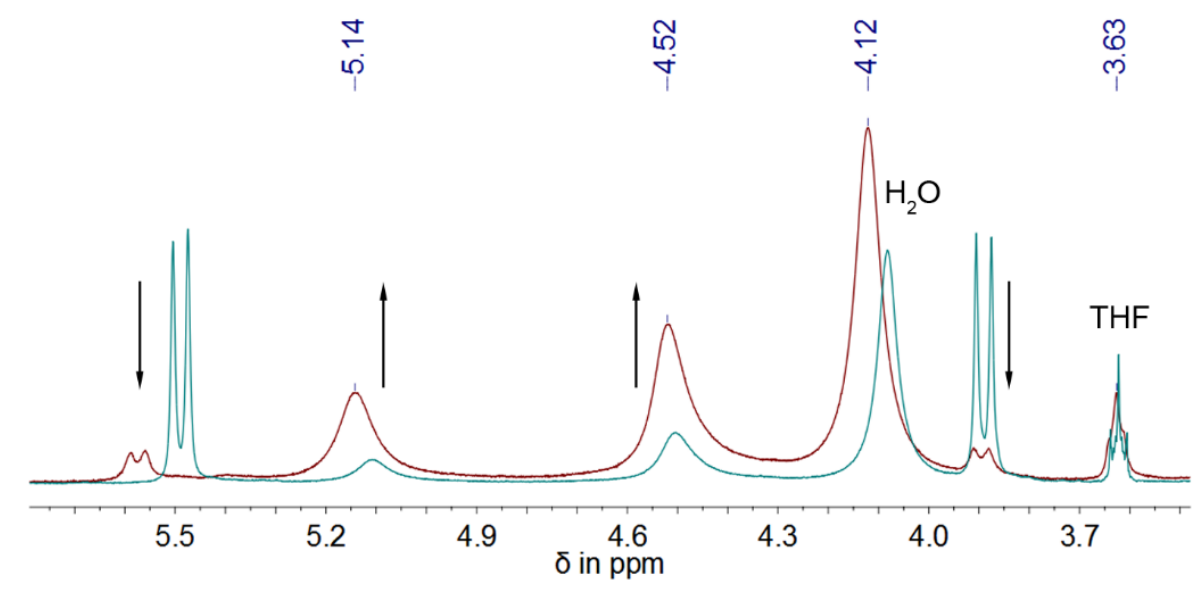

Figure 2.71: Extract of the superimposed ${ }^{1} \mathrm{H}$ NMR spectra of $\mathbf{1 7}$ without (turquois) and with addition of 10 eq $\mathrm{NaBr}$ (red) in DMF- $d_{7}$ at $243 \mathrm{~K}$. After the addition of the salt, the signal intensity of the diastereotopic methylene protons $\left(\mathrm{CH}_{2}\right)$ decreases, while two different signals at 5.14 and $4.52 \mathrm{ppm}$ rise.

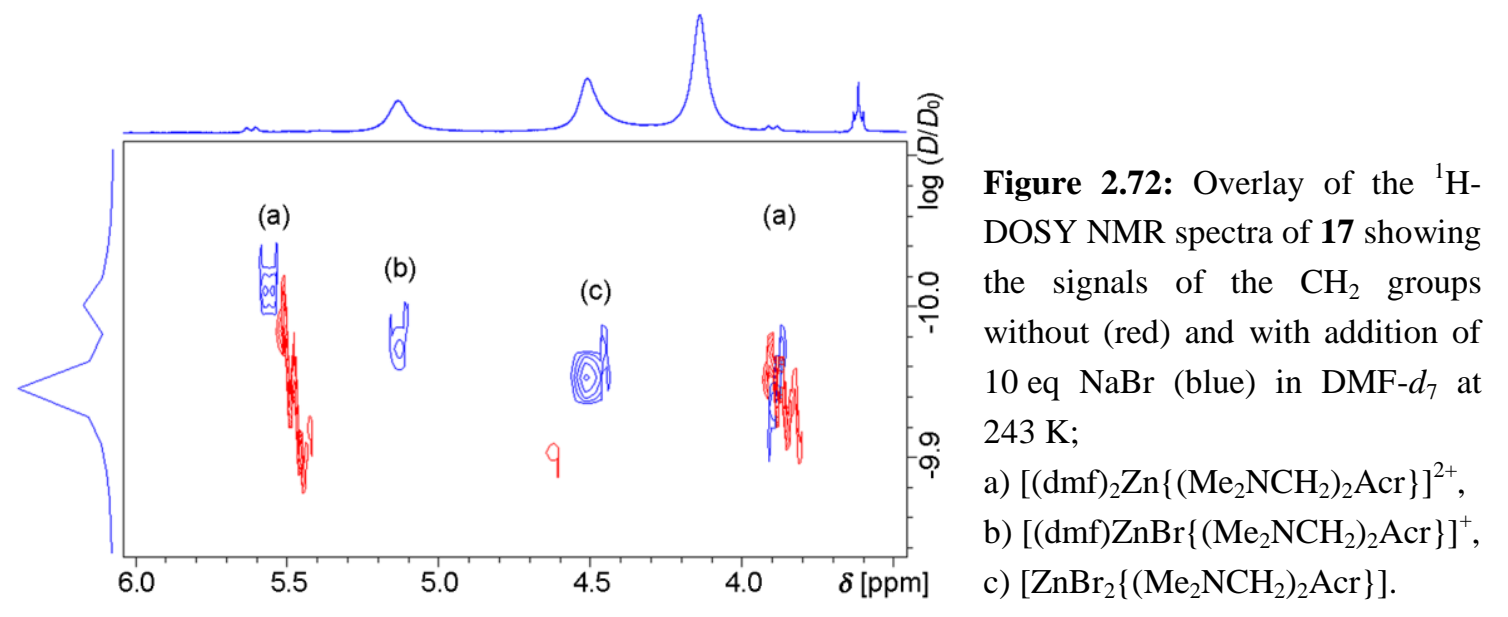


Additionally, tetramethylbutane (TMB) was added as internal reference for the ${ }^{1} \mathrm{H}-\mathrm{DOSY}$ NMR experiments. Its diffusion coefficient was kept constant to generate comparability of the different spectra. The diffusion coefficient of the rising signals decreases slightly from (a) to (c) which can be explained by the smaller radius/mass ratio of a bromine atom compared to a DMF molecule (van der Waals volume of bromine: $2.74^{-29} \mathrm{~m}^{3}$; DMF: $1.31^{-28} \mathrm{~m}^{3}$ ). ${ }^{[87,136]}$ Consequently, (c) should represent the dibrominated species (17). The new signal (b) has a diffusion coefficient that lies between the two others and is therefore assigned to the monobrominated compound (18). Furthermore, the signals at room temperature are no longer broadened after the addition of $\mathrm{NaBr}$ (Figure 7.2). Due to these results, the dynamic processes can be attributed to the exchange between bromide ions and solvent molecules.

Zinc ions are spectroscopically silent making it difficult to detect a ligand-metal interaction by NMR spectroscopy. However, the ${ }^{15} \mathrm{~N}$ NMR chemical shifts of the free ligand and of the zinc complex were measured using the ${ }^{1} \mathrm{H},{ }^{15} \mathrm{~N}-\mathrm{HMBC}$ experiment (Figure 7.5 and Figure 7.6, respectively). The comparison of the resulting shifts shows a small but significant deshielding of $\Delta \delta=8.6 \mathrm{ppm}^{[137]}$

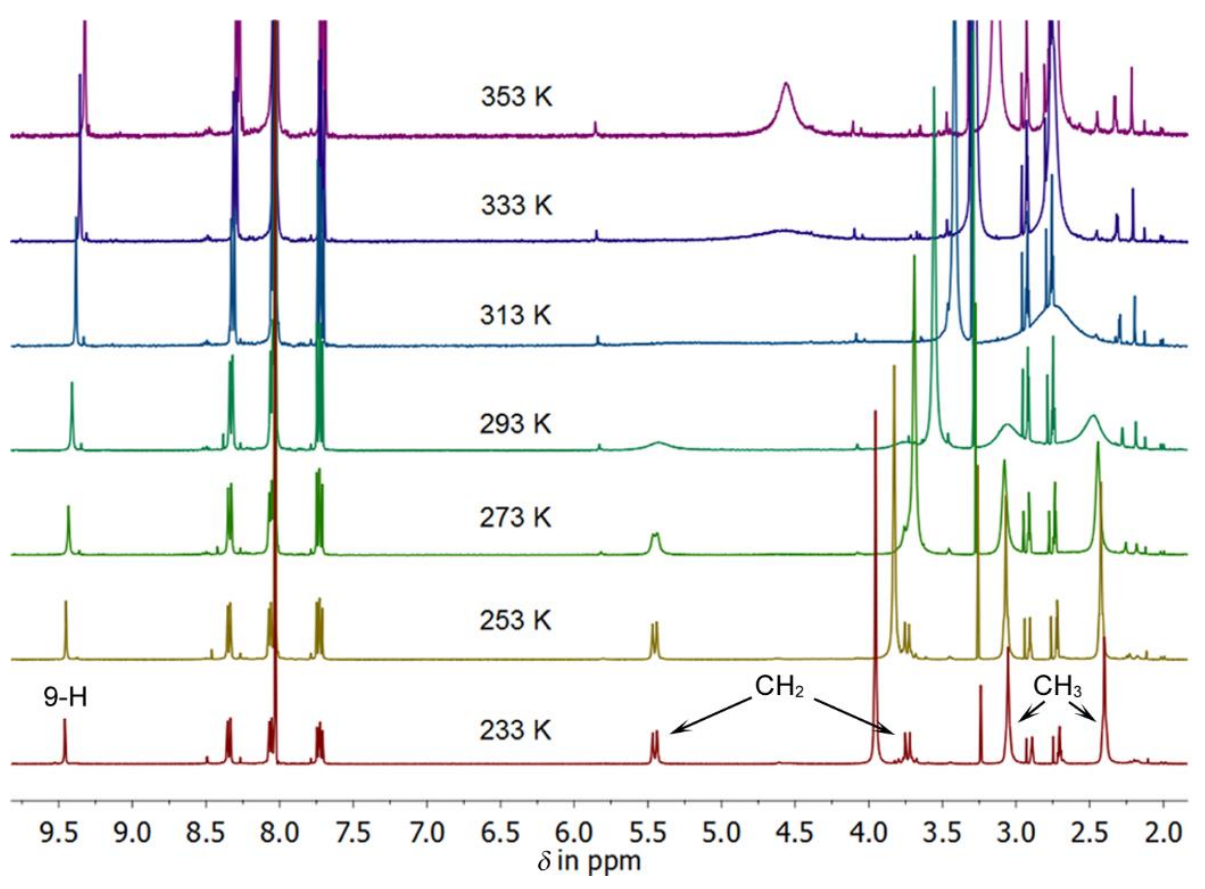

Figure 2.73: Temperature-dependent ${ }^{1} \mathrm{H}$ NMR spectra of 19 in DMF- $d_{7}$. The discussed signals are marked at $233 \mathrm{~K}$.

In principle, the ${ }^{1} \mathrm{H}$ NMR spectra of $\mathbf{1 9}$ show similar dependencies upon temperature variation as shown for compound 17 (Figure 2.73). The coalescence temperature in DMF- $d_{7}$ is around $313 \mathrm{~K}$ where the diastereotopicity of the $\mathrm{CH}_{2}$ protons becomes visible as well as the mentioned splitting of the signal of the methyl groups. But in this case no second set of signals appears and the spectrum is less broadened. Most likely, the soft Lewis acid $\mathrm{Cd}^{2+}$ does not exchange its large and soft bromides with the small oxygen donor DMF at all or at a smaller rate. However, the advantage of this complex is the NMR active ${ }^{113} \mathrm{Cd}$ nucleus. Employing a $2 \mathrm{D}{ }^{1} \mathrm{H},{ }^{113} \mathrm{Cd}-\mathrm{HMBC}$ experiment at low temperature, the identification of vicinal couplings of the methyl and methylene protons to the metal ion were possible (Figure 7.7). The chemical shift of the cadmium isotope is $-345 \mathrm{ppm}$, referenced to $\mathrm{Me}_{2} \mathrm{Cd}$. Interestingly, a coupling to the ${ }^{113} \mathrm{Cd}$ nucleus was only 
observed for one proton of each methylene group. The coupling to the other proton is likely not to be observed due to an unfavourable angle between the related atoms. ${ }^{[138]}$ From the NMR spectroscopic experiments it is clear that the complexes adopt the same contact ion pairs in solution as observed in the solid state.

Another analytical method which allows investigating the transferability of the solid state structure to solution is electrospray ionisation mass spectrometry. A time-of-flight (TOF) spectrometer was chosen because of the poor solubility of $\mathbf{1 7}$ and $\mathbf{1 9}$ in most of the common solvents (e.g. THF, $\mathrm{MeOH}, \mathrm{MeCN}$ ). The measurements were performed in pure THF for the zinc complex and in $\mathrm{THF} / \mathrm{H}_{2} \mathrm{O}$ for the cadmium complex due to the even lower solubility. However, both complexes were sufficiently present in solution for the TOF spectrometer. Figure 2.74 depicts two comparisons of the mass patterns of $\mathbf{1 7}$ and 19. The black lines represent the experimental data, whereas the red lines illustrate the simulated spectrum for the proposed structural motif (in the positive ion mode one bromide anion was omitted for the calculated spectrum).
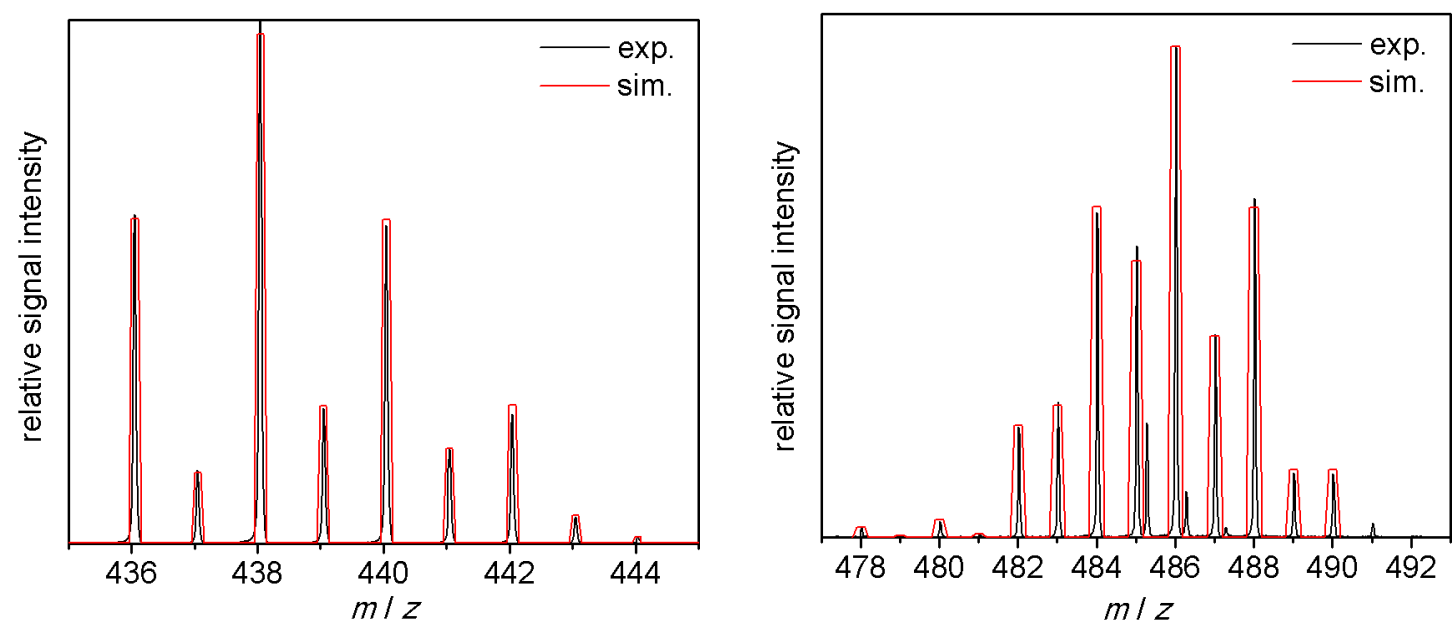

Figure 2.74: Parts of the mass spectra showing the simulated (red) and the experimental (black) isotope pattern of the cationic $\left[\left(\mathrm{C}_{19} \mathrm{H}_{23} \mathrm{~N}_{3}\right) \mathrm{ZnBr}\right]^{+}$(left) and $\left[\left(\mathrm{C}_{19} \mathrm{H}_{23} \mathrm{~N}_{3}\right) \mathrm{CdBr}\right]^{+}$(right).

The simulated isotope patterns were calculated using the COMPASS ${ }^{\circledR}$ software package from Bruker Daltonik. The comparisons demonstrate perfect matches of the predicted and observed species, which is also given in precise $m / z$ data in Table 2.13. Furthermore, the full mass spectrum is free of any ionic fragments because only signals generated by the acridine derivative, with and without metal ion, and related fragments can be detected (Figure 7.8).

Table 2.13: Measured and theoretical $\mathrm{m} / \mathrm{z}$ ratios of cations observed upon ESI-TOF-mass spectrometric analysis of $\mathbf{1 7}$ and $\mathbf{1 9}$ in THF or in a THF/ $\mathrm{H}_{2} \mathrm{O}$ mixture, respectively.

\begin{tabular}{c|cc|cc}
\hline & \multicolumn{2}{|c|}{$\mathbf{M = Z n ( 1 7 )}$} & \multicolumn{2}{c}{$\mathbf{M = C d ~ ( 1 9 )}$} \\
Cation & $m / z$ (measured) & $m / z$ (theoretical) & $m / z$ (measured) & $m / z$ (theoretical) \\
\hline \hline$\left[\left(\mathrm{C}_{19} \mathrm{H}_{23} \mathrm{~N}_{3}\right) \mathrm{H}\right]^{+}$ & 294.20 & 294.20 & 294.21 & 294.20 \\
{$\left[\left(\mathrm{C}_{19} \mathrm{H}_{23} \mathrm{~N}_{3}\right) \mathbf{M B r}^{+}\right.$} & 438.05 & 438.03 & 486.02 & 486.01 \\
{$\left[\left(\mathrm{C}_{19} \mathrm{H}_{23} \mathrm{~N}_{3}\right)_{2} \mathrm{HMBr}_{2}\right]^{+}$} & 813.15 & 813.15 & 861.15 & 861.12 \\
{$\left[\left(\mathrm{C}_{19} \mathrm{H}_{23} \mathrm{~N}_{3}\right)_{2} \mathbf{M}_{2} \mathrm{Br}_{3}\right]^{+}$} & 957.00 & 956.99 & 1050.95 & 1050.94 \\
\hline
\end{tabular}


The same is valid for the $\mathrm{Cd}^{2+}$ complex (Figure 7.9). Both methods, NMR spectroscopy and mass spectrometry, emphasise that the solid state structure is maintained in solution. Apart from the expected complexes shown in Figure 2.74, additional interesting species were found during the measurement. In Table 2.13, two dimeric complexes were identified. One consists of two ligands with an $\mathrm{MBr}_{2}$ centre and a significant abundance. The second is involving two ligand systems but also two metal ions with the molecular formula $\left[\left(\mathrm{C}_{19} \mathrm{H}_{23} \mathrm{~N}_{3}\right)_{2} \mathrm{M}_{2} \mathrm{Br}_{3}\right]^{+}$. In both metal ion cases, the abundance of the latter species was very low. However, with the help of a CID spectrum (collision induced dissociation) the mass pattern could be resolved precisely and its fragments could all be assigned to the used compounds. Additionally, the simulated spectra perfectly match the measured data which is true for all listed cations (Figure 7.10 and Figure 7.11). These complexes are most likely formed during the measurement, especially the aggregates with a high molecular mass. But the structure with the formula $\left[\left(\mathrm{C}_{19} \mathrm{H}_{23} \mathrm{~N}_{3}\right)_{2} \mathrm{HMBr}_{2}\right]^{+}$is conceivable in solution if the ligand to metal salt ratio would be increased. Further investigations with varied ratios would be an interesting study. 


\section{CONCLUSION AND OUTLOOK}

Two different fluorophores were investigated in this thesis on their potential usability as molecular sensors. A modified and greatly improved reaction procedure ${ }^{[64]}$ was established for the scarcely explored 5,10-dimethyl-2-azaanthracene (3). The methyl groups act as spacer units for further substituted amine receptors. Different from what was expected, their bromination was not feasible and resulted in the decomposition of the compound. Former substitution studies with the pure 2-azaanthracene were equally unsuccessful. ${ }^{[63]}$ Unless easy derivatisation is not guaranteed, this fluorophore is not consistent with the requirement for sensor devices.

The second approach was based on the heteroaromatic system acridine. In this case, a variety of new amine substituted ligands were synthesised and examined with special emphasis on their fluorescence properties. It was found that the introduction of an amine group in 4-position is not straightforward. Only a few publications are present considering the synthesis. Future investigations of 4-aminoacridine derivatives should focus on the very recently reported copper catalysed Ullmann coupling with a subsequent condensation reaction by the use of $\mathrm{POCl}_{3}$ (Scheme 3.1). ${ }^{[139]}$<smiles>[X]c1ccccc1C(=O)O</smiles><smiles>Nc1ccccc1[N+](=O)[O-]</smiles>

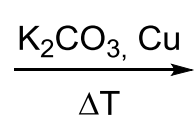<smiles>O=C(O)c1ccccc1Nc1ccccc1[N+](=O)[O-]</smiles>

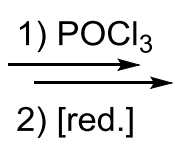<smiles>Nc1cccc2c(Cl)c3ccccc3nc12</smiles>

Scheme 3.1: Proposed reaction pathway for the synthesis of 4-amino-9-chloroacridine, inspired by the publication of Jiang and co-workers. ${ }^{[139]}$

More feasible than the monosubstitution was the introduction of two bromomethylene units which bromine atoms were subsequently substituted by secondary amines. The resulting new acridine derivatives (10-16) were characterised in detail by NMR and fluorescence spectroscopy. Apart from the oily TrMEDA derivatives $\mathbf{1 1}$ and 12, the solid state structures of all fluorophore-spacerreceptor systems were examined by X-ray diffraction. Their geometrical arrangements and the packing effects of the crystal structures were discussed and compared. The synthesised acridinyl amines respond differently towards the tested metal ions and show interesting behaviour in various solvents. For instance, an extraordinary excimer formation of $\mathbf{1 3}$ was observed by the use of a non-polar solvent at a defined concentration.

The PET based sensing abilities of the acridine systems were improved by the variation of the functionality of the amine side arms, resulting in the molecular sensor 4,5-bis $(\mathrm{N}, \mathrm{N}$ dimethylaminemethylene)acridine (15). Its fluorescence spectra show a remarkable metal ion selectivity and increase in fluorescence emission upon titration with dissolved $\mathrm{ZnBr}_{2}$ and $\mathrm{CdBr}_{2}$ in methanol (Figure 3.2). For the latter, even water can be used to dissolve the metal salt without influencing the detectability threshold. The high sensitivity of the acridine based sensor molecule and its good stability was demonstrated successfully. The very efficient electron transfer features 15 as a valuable ligand for medical or environmental purposes. Besides the detection of metal ions, the addition of acids and bases resulted in an instantaneously switch between the fluorescent on/off states by protonation/deprotonation of the amine receptors, repeatable without degeneration. Thus, $\mathbf{1 5}$ is as well applicable as a $\mathrm{pH}$ sensor. 


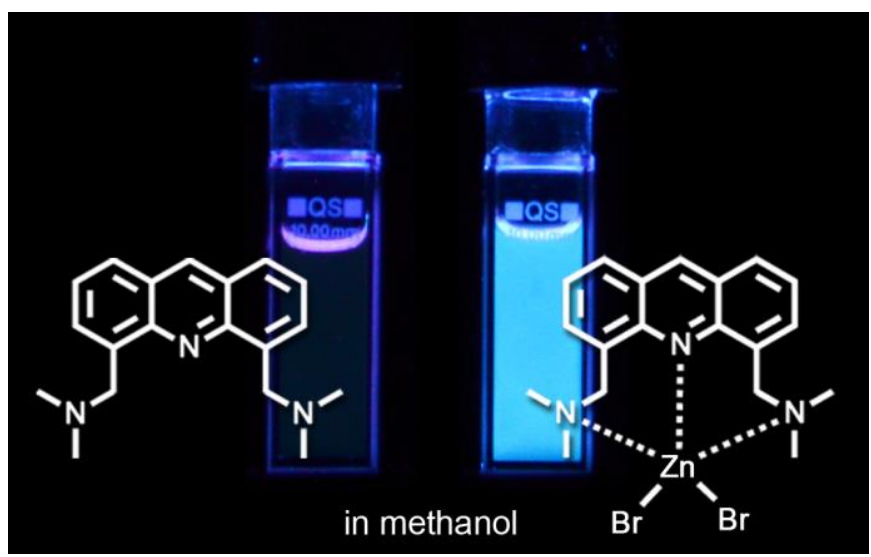

Figure 3.1: Fluorescence phenomenon of 15 in methanol $\left(10^{-5} \mathrm{M}\right.$, left) and with addition of $\mathrm{ZnBr}_{2}(1 \mathrm{eq}$, right). The cuvettes were irradiated with UV light at $365 \mathrm{~nm}$.
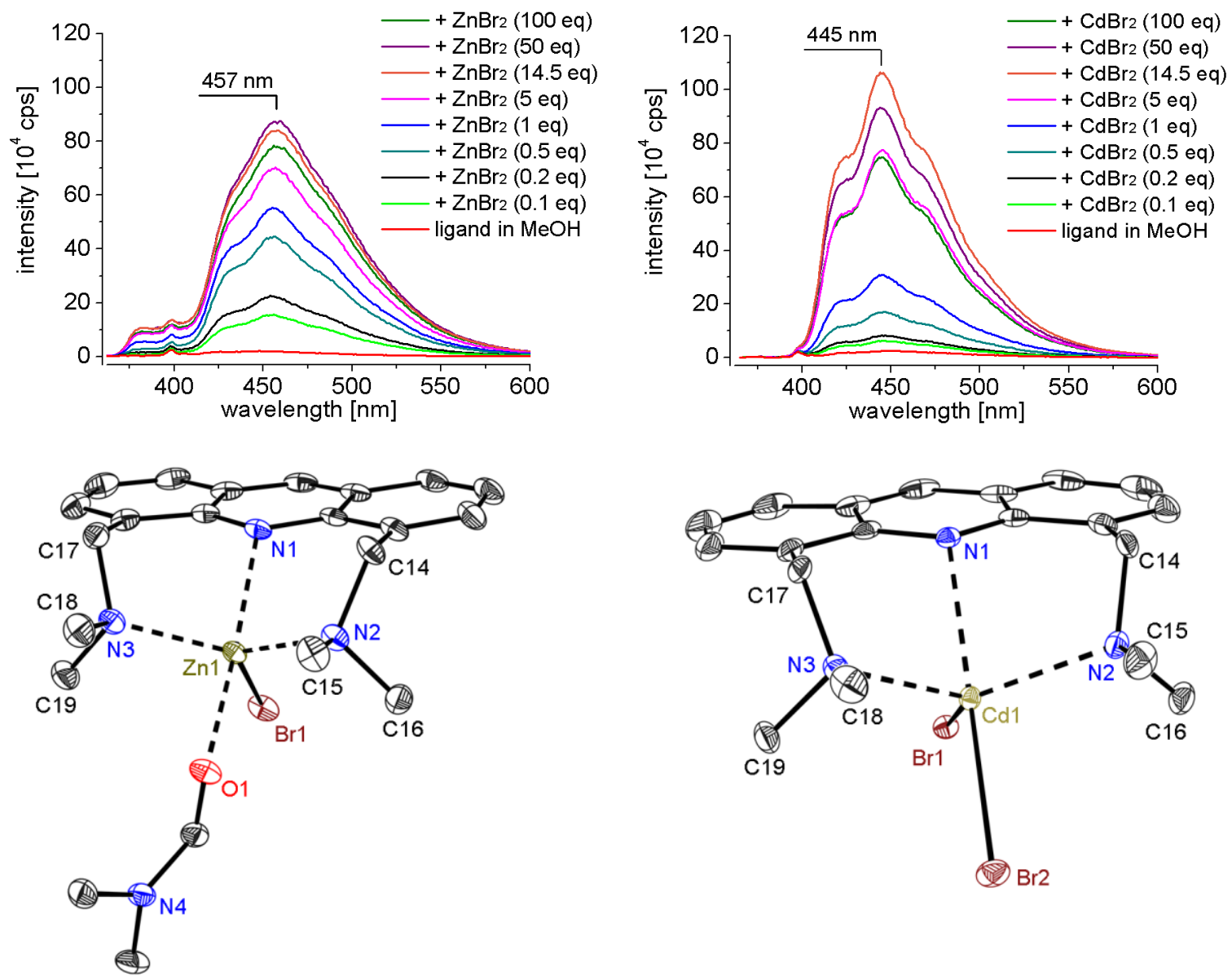

Figure 3.2: Titration experiment with dissolved $\mathrm{Zn}^{2+}$ and $\mathrm{Cd}^{2+}$ ions to a solution of $\mathbf{1 5}$ in methanol demonstrating the huge enhancement of the emission intensity (top). The corresponding solid state structures of the metal complexes $\mathbf{1 8}$ and $\mathbf{1 9}$ are depicted underneath.

The solid state contact ion pairs $\mathbf{1 8}$ and $\mathbf{1 9}$ determined by X-ray diffraction were confirmed to be present in solution by NMR spectroscopy and ESI mass spectrometry. Compared to the numerous publications concerning zinc sensors, only very few provide structural evidence of the metal ion coordination, although this is fundamental for the understanding of fluorescence processes. The 
solid state structures indicate a coordination of the $\mathrm{Zn}^{2+}$ and $\mathrm{Cd}^{2+}$ ions by all three nitrogen atoms, additionally proven by computational studies.

With respect to these computational results, the coordination of zinc is stronger than its heavier homologue cadmium which was supported by fluorescence measurements. To classify the strength of the chelation, the determination of the stability constant is of interest. Therefore, initial attempts have been made in the course of this thesis: Job plots ${ }^{[140]}$ were generated with values obtained from fluorescence titration experiments. The graphs indicated a ligand to metal coordination of 2:1 which is not in line with the results discussed above. Interestingly, complementary NMR experiments in the course of a master thesis stated a ligand to metal coordination of $1: 1$ and in some cases $1: 2 .{ }^{[141]}$ Due to the inconsistent results, a reliable value for the stability constant could not be calculated. This unexpected behaviour should be investigated in more detail.

In the case of zinc, spectroscopic studies concerning the influence of the anion were made. For instance, the exchange of bromide with acetate ions gave a tremendous increase of the fluorescence emission, reaching $95 \%$ of the intensity of pure anthracene (under the same experimental conditions). In comparison, the use of $\mathrm{ZnBr}_{2}$ yielded a value of $12 \%$. Additionally, the geometrical parameters of both solid state structures were discussed and compared with each other.

Fluorescence measurements in pure water were not feasible in the course of this thesis due to the water-insolubility of the organic ligand. However, according to the high demand for molecular sensors in medical applications, its solubility behaviour should be improved. The 9-position of the acridine ring is suitable for the introduction of functional groups. This was shown with the synthesis of 9-methyl- (4) and 9-bromomethyleneacridine (5). These compounds can easily be modified by oxidation or substitution reactions. For instance, Scheme 3.2 illustrates two synthetic routes to 9-carboxyacridine whose polar group strongly enhances the water-solubility of acridine.

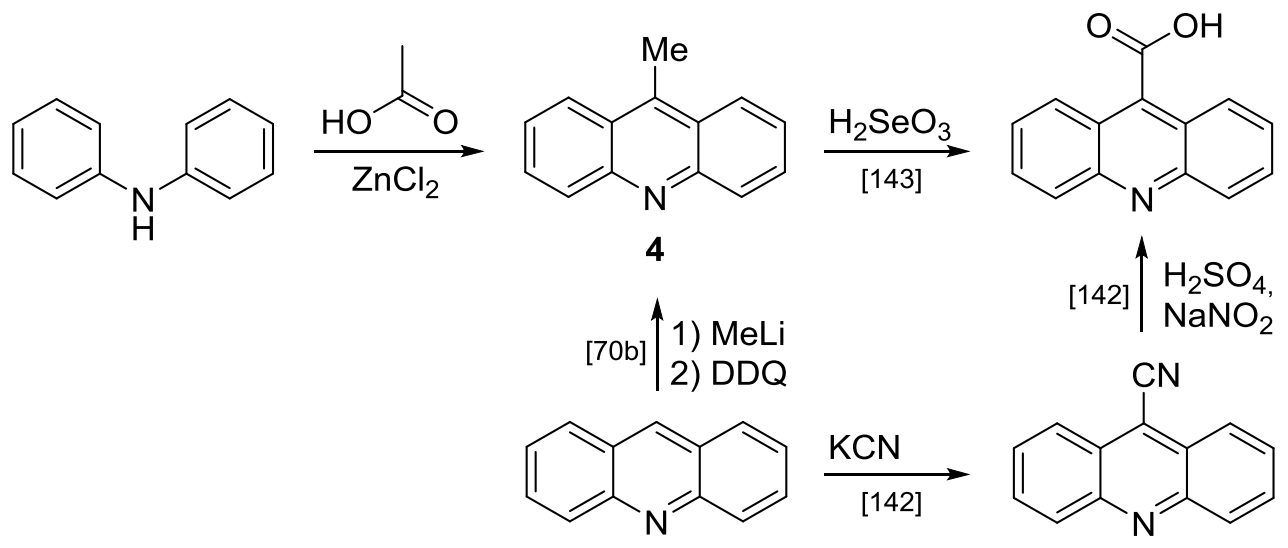

Scheme 3.2: Synthesis of 9-carboxyacridine by two different reaction pathways. ${ }^{\text {[70b] [142] [143] }}$

The reaction pathway via 9-cyanoacridine was already established in the Stalke group in 2015. ${ }^{[144]}$ This intermediate could also be of interest for further studies since the strong electronwithdrawing cyano group is known to shift the emission of fluorophores to longer wavelengths. ${ }^{[145]}$ Thus, the emitting range of the compounds lie more in the visible region of the electromagnetic spectrum and is easily detectable by the naked eye. 



\section{EXPERIMENTAL SETTINGS}

\subsection{General working procedure}

Air and moisture sensitive reactions were carried out using modified Schlenk ${ }^{[146]}$ techniques in dried nitrogen or argon atmosphere and resulting sensitive substances were stored in an argon glovebox. The used solvents were purchased in high quality and dried due to standard laboratory techniques if necessary. For the fluorescence measurements, water was purified first through a Millipore water purification system Milli-RO 3 plus and finally with a Millipore ultrapure water system Milli-Q plus 185. All employed reactants were commercially available or reproduced according to the given literature procedure.

\subsection{Applied analytical methods}

\subsubsection{Computational studies}

For the geometry optimisations of 11, the basis Ahlrichs-TZV/def2-TZV ${ }^{[131 e, ~ 147] ~ w e r e ~ u s e d ~ f o r ~}$ calculations (including the atom-pairwise dispersion correction ${ }^{[131 f, 148]}$ with the Becke-Johnson damping scheme (D3BJ ${ }^{[149]}$ ). The LIBINT2 library ${ }^{[150]}$ was used for the computation of twoelectron integrals. The geometry optimisations of 17-19 were carried out at the B3LYP-D3/def2$\mathrm{TZVPP}^{[131,151]}$ level of theory (including Becke-Jones type damping ${ }^{[149]}$ of the dispersion correction $^{[131]]}$ ). In the case of Cd, the Stuttgart/Dresden ECP28MDF ${ }^{[152]}$ was used. The electronic densities and the corresponding NBO analysis ${ }^{[132-133]}$ were computed at the same level. The B3LYP calculations were performed under the RIJCOSX approximation. ${ }^{[153]}$

All calculations $(\mathbf{1 1}, \mathbf{1 7}-\mathbf{1 9})$ were realised with the $\mathrm{ORCA}^{[109]}$ program package.

\subsubsection{Elemental analysis}

Elemental analyses were carried out by the Analytische Labor des Instituts für Anorganische Chemie der Georg-August-Universität Göttingen. The determination of the organic elements (C, $\mathrm{H}, \mathrm{N}, \mathrm{S}$ ) were realised with an Elementar Vario EL3 and the metal ions were analysed with the AAS 5FL Analytik Jena. The amount of halides $(\mathrm{Cl}, \mathrm{Br})$ was determined potentiometric or titrimetric.

\subsubsection{Fluorescence spectroscopy}

The fluorescence measurements were performed on a Horiba Jobin-Yvon Fluoromax-4 spectrometer. A general setup of a spectrofluorometer is depicted in Figure 4.1. The Fluoromax-4 is equipped with a $150 \mathrm{~W}$ xenon arc lamp (1) which provides a continuous spectrum of light. This is focused on the entrance slit of the Czerny-Turner excitation monochromator (2) by elliptical mirrors. The beam with the selected wavelength is split and a small partition of light is directed to a reference detector (4). The main part of the beam hits the sample which perpendicular emitted light is directed to the emission monochromator (5). From here, the signal is detected by a photomultiplier (6). 


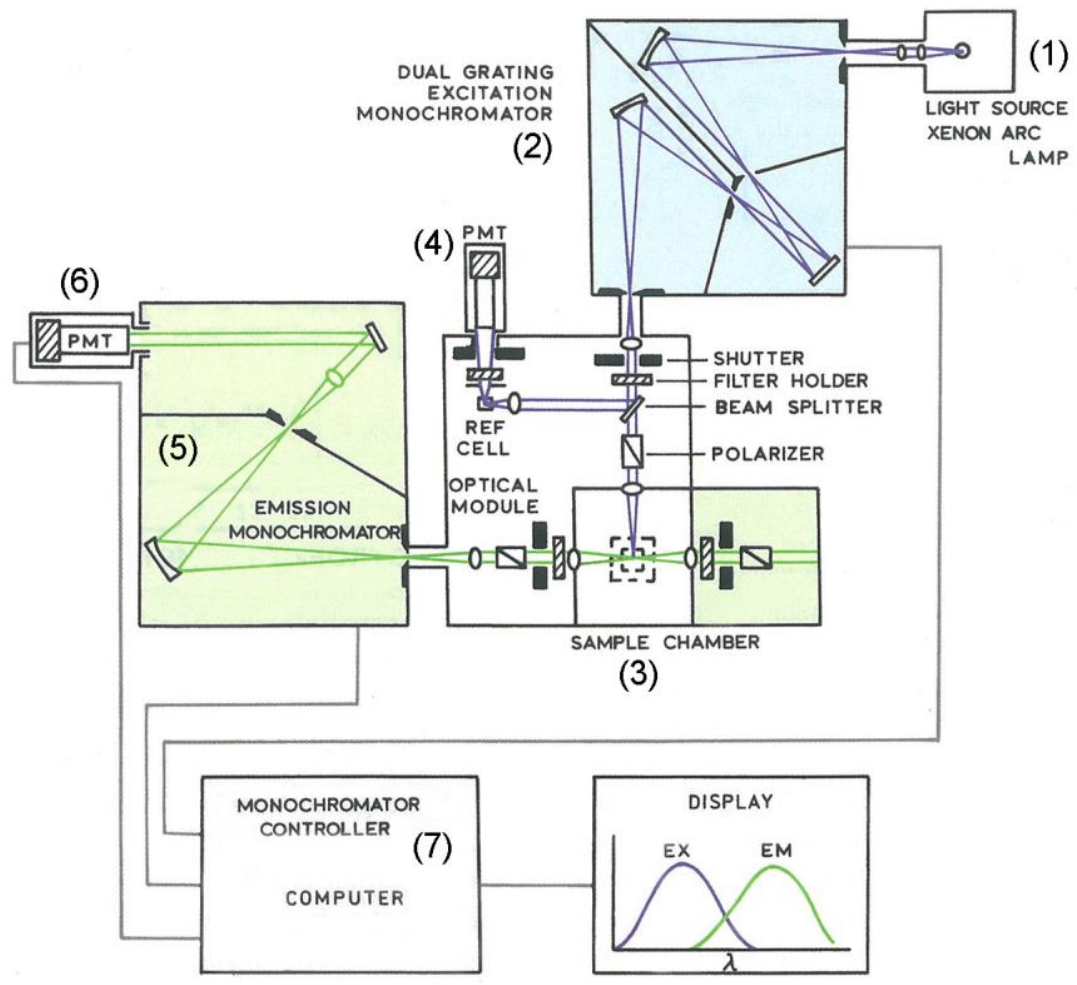

Figure 4.1: General setup of a spectrofluorometer, illustrating the beam path. ${ }^{[154]}$

The spectra were recorded and modified graphically using the software FluorEssence ${ }^{\mathrm{TM}}$ v.3.0 at a computer terminal (7). ${ }^{[155]}$ The entrance and exit slit widths of each monochromator can be adjusted to vary the amount of passing light. If the slits are wider, a more intense spectrum is obtained but with loss of resolution. On the one hand, the overflow of the detector due to too strong emitting samples can be prevented. On the other hand, the signal-to-noise ratio of low fluorescent compounds can be improved.

\subsubsection{Mass spectrometry}

Most of the mass spectrometry experiments were carried out on a MAT $95(70 \mathrm{eV})$ instrument for EI-MS ${ }^{[156]}$ experiments as well as on a HCT Ultra instrument for ESI-MS ${ }^{[157]}$ experiments. For the electrospray ionisation mass spectrometric studies of $\mathbf{1 7}$ and $\mathbf{1 9}$, sample solutions of $c \approx 5 \mathrm{mM}$ were continuously administered into the ESI source of a micrOTOF-QII mass spectrometer (Bruker Daltonik) by a step motor-driven gas-tight syringe (flow rate: $0.5 \mathrm{ml} / \mathrm{h}$ ). This instrument combines a quadrupole mass filter with a time-of-flight analyser. The simulated isotope patterns were calculated using the COMPASS ${ }^{\circledR}$ software package from Bruker Daltonik. The mass to charge ratios of the molecular ions and the fragment ions are based on the isotopes bearing the highest natural abundances $\left({ }^{1} \mathrm{H},{ }^{13} \mathrm{C},{ }^{14} \mathrm{~N},{ }^{16} \mathrm{O},{ }^{32} \mathrm{~S},{ }^{64} \mathrm{Zn},{ }^{114} \mathrm{Cd},{ }^{79} \mathrm{Br} /{ }^{81} \mathrm{Br}\right)$.

\subsubsection{NMR spectroscopy}

The NMR measurements were performed on a Bruker Avance III 300 and Bruker Avance III HD 400 spectrometer. Unless it was stated otherwise, the measurements were carried out at room temperature in 1-10\% solutions of deuterated solvents. The chemical shifts $(\delta)$ are reported in parts per million (ppm) and the coupling constants $J$ in $\mathrm{Hz}$. The residual proton signals of the 
incompletely deuterated solvents were chosen as internal standards for ${ }^{1} \mathrm{H}$ NMR spectra. ${ }^{[158]}$ For

${ }^{13} \mathrm{C}$ spectra, the carbon resonances of the solvents were used for calibration. The assignment of the peaks was accomplished by two dimensional NMR techniques $\left({ }^{1} \mathrm{H},{ }^{1} \mathrm{H}\right.$-COSY, ${ }^{1} \mathrm{H},{ }^{13} \mathrm{C}-\mathrm{HSQC}$, ${ }^{1} \mathrm{H},{ }^{13} \mathrm{C}-\mathrm{HMBC}$ ). In the case of $\mathbf{1 7}$ and $\mathbf{1 9}$, further specialised experiments were used for the evidence of ligand-metal interactions ( ${ }^{1} \mathrm{H}-\mathrm{DOSY},{ }^{1} \mathrm{H},{ }^{15} \mathrm{~N}-\mathrm{HMBC},{ }^{1} \mathrm{H},{ }^{113} \mathrm{Cd}-\mathrm{HMBC}$ ). The observed multiplicities are abbreviated as follows: $\mathrm{s}=$ singlet, $\mathrm{d}=$ doublet, $\mathrm{t}=$ triplet, $\mathrm{q}=$ quartet, $\mathrm{m}=$ multiplet. Combined abbreviations are derived from their components (e.g. $\mathrm{dd}=$ doublet of doublets).

\subsubsection{UV/Vis spectroscopy}

Absorption spectra (UV/vis) of $\mathbf{1 3}$ were recorded at a Jasco V-650 double beam spectrophotometer and of all other compounds at an Agilent 8453 diode array spectrophotometer. The samples contained $10^{-5} \mathrm{M}$ solutions in methanol. The data were corrected by subtraction of a reference measurement of the pure solvent. In contrast to the fluorescence experiments, the detector is placed behind the cuvette, in linear arrangement to the beam. The transmitting light which is not absorbed by the sample solution is detected. Non-radiative processes can be observed in this way.

\subsection{Synthesis and characterisation}

\subsubsection{Synthesis of 1-phenoxycarbonyl-3-acetyl-4-(phenylethyl)-1,4-dihydropyridine} (1)

1-Bromo-1-phenylethane ( $3.65 \mathrm{ml}, 26.4 \mathrm{mmol}, 1.0$ eq.) was added over $5 \mathrm{~min}$ to a suspension of zinc dust (2.58 g, $39.5 \mathrm{mmol}, 1.5 \mathrm{eq}$.) in THF ( $30 \mathrm{~mL})$ and was stirred at room temperature for $4 \mathrm{~h}$. Separately, to a solution of 3-acetylpyridine ( $4.35 \mathrm{~g}, 35.9 \mathrm{mmol}, 1.4$ eq.) in THF (45 ml) phenylchloro formiate (5.63 g, $35.9 \mathrm{mmol}, 1.4$ eq.) was added slowly at room temperature and the mixture was stirred for one hour.

After completion of the reactions, the organozinc compound was given dropwise to the activated pyridine derivative at $0{ }^{\circ} \mathrm{C}$, where it was stirred for $60 \mathrm{~min}$. The reaction mixture was quenched with aqueous $\mathrm{NH}_{4} \mathrm{Cl}$ solution $(20 \%, 15 \mathrm{ml})$ and extracted with EA (2x $\left.100 \mathrm{ml}\right)$. The organic phase was washed with saturated, aqueous $\mathrm{Na}_{2} \mathrm{CO}_{3}$ solution $(60 \mathrm{ml})$ and dem. water $(40 \mathrm{ml})$ and was dried over $\mathrm{MgSO}_{4}$. The pure product was filtrated and the solvent was removed under reduced pressure. $1(5.38 \mathrm{~g}, 15.5 \mathrm{mmol}, 59 \%)$ could be obtained after purification by column chromatography (PE/EA, 10:1) as a yellow oil. The diastereomeric ratio of the two formed compounds is approximately $3: 1$.

Chemical formula: $\quad \mathrm{C}_{22} \mathrm{H}_{21} \mathrm{NO}_{3}$

Molecular weight: $\quad 347.41 \mathrm{~g} / \mathrm{mol}$

DC $\left(\mathrm{SiO}_{2}\right): \quad \quad R_{\mathrm{f}}=0.30 / 0.24(\mathrm{PE} / \mathrm{EA}, 10: 1)$<smiles>CC1c2ccccc2C(=O)C2=CN(C(=O)Oc3ccccc3)C=CC21</smiles> 


\section{${ }^{1}$ H NMR}

$\left(300 \mathrm{MHz}, \mathrm{CDCl}_{3}\right): \quad$ main product: $\delta[\mathrm{ppm}]=7.68\left(\mathrm{~s}, 1 \mathrm{H}, \mathrm{H}_{5}\right), 7.49-6.86\left(\mathrm{~m}, 10 \mathrm{H}, \mathrm{H}_{\mathrm{Ph}}\right)$, 6.86-6.78 (m, $\left.1 \mathrm{H}, \mathrm{H}_{2}\right), 5.32\left(\mathrm{br}, 1 \mathrm{H}, \mathrm{H}_{3}\right), 3.76\left(\mathrm{dd},{ }^{3} \mathrm{~J}=10.2,4.8 \mathrm{~Hz}\right.$ $\left.1 \mathrm{H}, \mathrm{H}_{4}\right), 3.04\left(\mathrm{dq},{ }^{3} J=7.2,4.8 \mathrm{~Hz}, 1 \mathrm{H}, \mathrm{H}_{6}\right), 2.32\left(\mathrm{~s}, 3 \mathrm{H}, \mathrm{COCH}_{3}\right), 1.30$ $\left(\mathrm{d},{ }^{3} \mathrm{~J}=7.2 \mathrm{~Hz}, 3 \mathrm{H}, \mathrm{CH}_{3}\right)$.

side product: $\delta[\mathrm{ppm}]=8.06\left(\mathrm{~s}, 1 \mathrm{H}, \mathrm{H}_{5}\right), 7.49-6.86\left(\mathrm{~m}, 10 \mathrm{H}, \mathrm{H}_{\mathrm{Ph}}\right)$, 6.86-6.78 (m, $\left.1 \mathrm{H}, \mathrm{H}_{2}\right), 4.88$ (br, $\left.1 \mathrm{H}, \mathrm{H}_{3}\right)$, 3.81-3.71 (m, $\left.1 \mathrm{H}, \mathrm{H}_{4}\right)$, 3.27$3.16\left(\mathrm{~m}, 1 \mathrm{H}, \mathrm{H}_{6}\right), 2.37$ (s, $\left.3 \mathrm{H}, \mathrm{COCH}_{3}\right), 1.18\left(\mathrm{~d},{ }^{3} \mathrm{~J}=7.2 \mathrm{~Hz}, 3 \mathrm{H}, \mathrm{CH}_{3}\right)$.

${ }^{13}$ C NMR

$\left(75 \mathrm{MHz}, \mathrm{CDCl}_{3}\right)$ : main product: $\delta[\mathrm{ppm}]=196.97\left(1 \mathrm{C}, \underline{\mathrm{COCH}}_{3}\right), 156.13(1 \mathrm{C}, \underline{\mathrm{COPh}})$ $134.85\left(1 \mathrm{C}, \mathrm{C}_{5}\right), 110.84\left(1 \mathrm{C}, \mathrm{C}_{3}\right), 37.86\left(1 \mathrm{C}, \mathrm{C}_{4}\right), 44.12\left(1 \mathrm{C}, \mathrm{C}_{6}\right)$, $25.37\left(1 \mathrm{C}, \mathrm{CO}^{\mathrm{C}} \mathrm{H}_{3}\right), 17.44\left(1 \mathrm{C}, \underline{\mathrm{CH}}_{3}\right)$.

side product: $\delta[\mathrm{ppm}]=196.97\left(1 \mathrm{C}, \underline{\mathrm{COCH}_{3}}\right), 156.13(1 \mathrm{C}, \underline{\mathrm{COPh}})$, $134.85\left(1 \mathrm{C}, \mathrm{C}_{5}\right), 110.15\left(1 \mathrm{C}, \mathrm{C}_{3}\right), 39.09\left(1 \mathrm{C}, \mathrm{C}_{4}\right), 41.53\left(1 \mathrm{C}, \mathrm{C}_{6}\right)$, $25.44\left(1 \mathrm{C}, \mathrm{CO} \underline{\mathrm{C}} \mathrm{H}_{3}\right), 13.31\left(1 \mathrm{C}, \underline{\mathrm{CH}}_{3}\right)$.

The carbon atoms of the phenyl groups could not be identified due to a too crowded spectrum.

\subsubsection{Synthesis of 3-acetyl-4-(phenylethyl)pyridine (2)}

1 (160 mg, $0.46 \mathrm{mmol}, 1.0$ eq.) was converted slowly with conc. $\mathrm{HNO}_{3}(30 \mathrm{ml})$ at $0{ }^{\circ} \mathrm{C}$ and stirred for $45 \mathrm{~min}$ at room temperature. For neutralisation, the reaction mixture was poured onto ice and treated with $\mathrm{K}_{2} \mathrm{CO}_{3}$. After extraction with DCM (3x $\left.30 \mathrm{ml}\right)$, it was washed with dem. water ( $2 \mathrm{x}$ $30 \mathrm{ml}$ ) and dried over $\mathrm{MgSO}_{4}$. The title compound (98 $\mathrm{mg}, 0.43 \mathrm{mmol}, 94 \%$ ) was obtained after a column chromatographic work-up (PE/EA, 10:1) as a dark-red oil.

$\begin{array}{ll}\text { Chemical formula: } & \mathrm{C}_{15} \mathrm{H}_{15} \mathrm{NO} \\ \text { Molecular weight: } & 225.29 \mathrm{~g} / \mathrm{mol} \\ \text { DC }\left(\mathrm{SiO}_{2}\right): & R_{\mathrm{f}}=0.45(\mathrm{PE} / \mathrm{EA}, 10: 1)\end{array}$

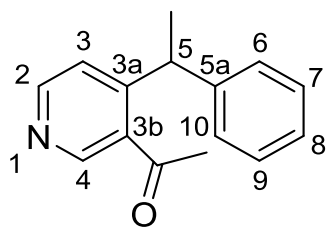

${ }^{1}$ H NMR

$\left(500 \mathrm{MHz}, \mathrm{CDCl}_{3}\right): \quad \delta[\mathrm{ppm}]=8.80\left(\mathrm{~s}, 1 \mathrm{H}, \mathrm{H}_{4}\right), 8.61\left(\mathrm{~d},{ }^{3} J=5.3 \mathrm{~Hz}, 1 \mathrm{H}, \mathrm{H}_{2}\right), 7.33-7.28(\mathrm{~m}$, $\left.3 \mathrm{H}, \mathrm{H}_{3}, \mathrm{Ph}\right), 7.25-7.15\left(\mathrm{~m}, 3 \mathrm{H}, \mathrm{H}_{\mathrm{Ph}}\right), 4.98\left(\mathrm{q},{ }^{3} J=7.1 \mathrm{~Hz}, 1 \mathrm{H}, \mathrm{H}_{5}\right), 2.46$ (s, $\left.3 \mathrm{H}, \mathrm{COC}_{3}\right), 1.63\left(\mathrm{~d},{ }^{3} \mathrm{~J}=7.1 \mathrm{~Hz}, 3 \mathrm{H}, \mathrm{CH}_{3}\right)$.

\section{${ }^{13}$ C NMR}

$\left(125 \mathrm{MHz}, \mathrm{CDCl}_{3}\right): \quad \delta[\mathrm{ppm}]=201.36(1 \mathrm{C}, \mathrm{C}=\mathrm{O}), 155.31\left(1 \mathrm{C}, \mathrm{C}_{3 \mathrm{a}}\right), 151.72\left(1 \mathrm{C}, \mathrm{C}_{2}\right), 148.92$ $\left(1 \mathrm{C}, \mathrm{C}_{4}\right), 144.36\left(1 \mathrm{C}, \mathrm{C}_{5 \mathrm{a}}\right), 134.43\left(1 \mathrm{C}, \mathrm{C}_{3 \mathrm{~b}}\right), 128.65\left(2 \mathrm{C}, \mathrm{C}_{7,9}\right), 128.21$ $\left(2 \mathrm{C}, \mathrm{C}_{6,10}\right), 126.69\left(1 \mathrm{C}, \mathrm{C}_{8}\right), 123.40\left(1 \mathrm{C}, \mathrm{C}_{3}\right), 39.45\left(1 \mathrm{C}, \mathrm{C}_{5}\right), 30.44$ (1 C, $\left.\mathrm{CO}^{-} H_{3}\right), 21.33\left(1 \mathrm{C}, \underline{\mathrm{CH}}_{3}\right)$. 


\subsubsection{Synthesis of 5,10-dimethyl-2-azaanthracene (3)}

2 (106 mg, $0.47 \mathrm{mmol}, 1.0 \mathrm{eq})$ was dissolved in MSA $(2 \mathrm{ml})$ and stirred for $24 \mathrm{~h}$ at room temperature. The reaction mixture was poured onto ice, neutralised with aqueous $\mathrm{NaHCO}_{3}$ solution and extracted with DCM (3x $25 \mathrm{ml}$ ). The combined organic phases were dried over $\mathrm{MgSO}_{4}$, filtrated and the solvent was removed under reduced pressure. A column chromatographic work-up (PE/EA, 1:1 $\rightarrow$ 1:3) yielded the desired product $(90 \mathrm{mg}, 0.43 \mathrm{mmol}$, $92 \%$, obtained as a yellow solid.

Chemical formula: $\quad \mathrm{C}_{15} \mathrm{H}_{13} \mathrm{~N}$

Molecular weight: $\quad 207.27 \mathrm{~g} / \mathrm{mol}$

$\mathrm{DC}\left(\mathrm{SiO}_{2}\right): \quad R_{\mathrm{f}}=0.15(\mathrm{PE} / \mathrm{EA}, 1: 1)$

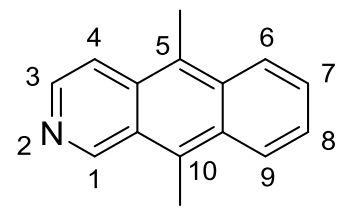

${ }^{1}$ H NMR

$\left(300 \mathrm{MHz}, \mathrm{CDCl}_{3}\right): \quad \delta[\mathrm{ppm}]=9.81\left(\mathrm{~s}, 1 \mathrm{H}, \mathrm{H}_{1}\right), 8.44\left(\mathrm{~d},{ }^{3} J=6.4 \mathrm{~Hz}, 1 \mathrm{H}, \mathrm{H}_{3}\right), 8.34(\mathrm{dd}$, $\left.{ }^{3} J=7.9,{ }^{4} J=1.7 \mathrm{~Hz}, 1 \mathrm{H}, \mathrm{H}_{6} / \mathrm{H}_{9}\right), 8.29\left(\mathrm{dd},{ }^{3} J=8.1,{ }^{4} J=1.8 \mathrm{~Hz}, 1 \mathrm{H}\right.$, $\left.\mathrm{H}_{6} / \mathrm{H}_{9}\right), 7.97\left(\mathrm{~d},{ }^{3} J=6.4 \mathrm{~Hz}, 1 \mathrm{H}, \mathrm{H}_{4}\right), 7.66-7.53\left(\mathrm{~m}, 2 \mathrm{H}, \mathrm{H}_{7,8}\right), 3.16(\mathrm{~s}$, $\left.3 \mathrm{H}, \mathrm{CH}_{3}\right), 3.00\left(\mathrm{~s}, 3 \mathrm{H}, \mathrm{CH}_{3}\right)$.

${ }^{13}$ C NMR

$\left(75 \mathrm{MHz}, \mathrm{CDCl}_{3}\right): \quad \delta[\mathrm{ppm}]=151.71\left(1 \mathrm{C}, \mathrm{C}_{1}\right), 138.82\left(1 \mathrm{C}, \mathrm{C}_{3}\right), 132.83\left(1 \mathrm{C}, \mathrm{C}_{\text {quart }}\right), 131.98$ $\left(1 \mathrm{C}, \mathrm{C}_{\text {quart }}\right), 130.81$ (1 C, $\left.\mathrm{C}_{\text {quart }}\right), 130.67$ (1 C, $\left.\mathrm{C}_{\text {quart }}\right), 128.05$ (1 C, $\left.\mathrm{C}_{\text {quart }}\right)$, $127.28\left(1 \mathrm{C}, \mathrm{C}_{7} / \mathrm{C}_{8}\right), 125.83\left(1 \mathrm{C}, \mathrm{C}_{7} / \mathrm{C}_{8}\right), 125.80\left(1 \mathrm{C}, \mathrm{C}_{6} / \mathrm{C}_{9}\right), 125.44$ $\left(1 \mathrm{C}, \mathrm{C}_{6} / \mathrm{C}_{9}\right), 124.72\left(1 \mathrm{C}, \mathrm{C}_{\text {quart }}\right), 117.71\left(1 \mathrm{C}, \mathrm{C}_{4}\right), 13.74\left(1 \mathrm{C}, \underline{\mathrm{CH}_{3}}\right)$, $13.50\left(1 \mathrm{C}, \underline{\mathrm{CH}}_{3}\right)$.

\section{EI MS}

$m / z(\%): \quad \quad \mathrm{m} / \mathrm{z}(\%)=207(50)[\mathrm{M}]^{+\bullet}, 192(100)\left[\mathrm{M}-\mathrm{CH}_{3}\right]^{+\bullet}, 178(6)\left[\mathrm{M}-\left(\mathrm{CH}_{3}\right)_{2}\right]^{+\bullet}$.

\subsubsection{Synthesis of 9-methylacridine (4)}

Diphenylamine (20.08 g, $118.6 \mathrm{mmol}, 1.0 \mathrm{eq})$ and zinc chloride $(80.0 \mathrm{~g}, 587.0 \mathrm{mmol}, 4.9 \mathrm{eq})$ was treated with glacial acetic acid $(25 \mathrm{ml}, 437.1 \mathrm{mmol}, 3.7 \mathrm{eq})$. The reaction mixture was stirred for one day at an oil bath temperature of $200{ }^{\circ} \mathrm{C}$. After cooling to room temperature, aqueous $2 \mathrm{M}$ $\mathrm{NaOH}$ solution was added and the product was extracted with DCM $(4 \mathrm{x} 600 \mathrm{ml})$. The gathered organic phases were dried over $\mathrm{MgSO}_{4}$, filtrated and the solvent was removed under removed pressure. The product $(13.1 \mathrm{~g}, 67.8 \mathrm{mmol}, 57.2 \%)$ could be obtained as a yellow powder.

Chemical formula: $\quad \mathrm{C}_{14} \mathrm{H}_{12} \mathrm{~N}$

Molecular weight: $\quad 193.22 \mathrm{~g} / \mathrm{mol}$

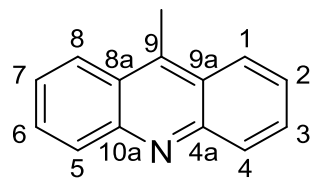


DC $\left(\mathrm{SiO}_{2}\right): \quad \quad R_{\mathrm{f}}=0.35(\mathrm{PE} / \mathrm{EA}, 30: 1)$

${ }^{1}$ H NMR

$\left(300 \mathrm{MHz}, \mathrm{CDCl}_{3}\right): \quad \delta[\mathrm{ppm}]=8.29-8.21\left(\mathrm{~m}, 4 \mathrm{H}, \mathrm{H}_{1,8,4,5}\right), 7.77\left(\mathrm{ddd},{ }^{3} J=8.7 \mathrm{~Hz}, 6.6 \mathrm{~Hz}\right.$, $\left.{ }^{4} J=1.2 \mathrm{~Hz}, 2 \mathrm{H}, \mathrm{H}_{3,6}\right), 7.56\left(\mathrm{ddd},{ }^{3} J=8.7 \mathrm{~Hz}, 6.6 \mathrm{~Hz},{ }^{4} J=1.2 \mathrm{~Hz}, 2 \mathrm{H}\right.$, $\left.\mathrm{H}_{2,7}\right), 3.13\left(\mathrm{~s}, 3 \mathrm{H}, \mathrm{C}_{3}\right)$.

${ }^{13}$ C NMR

$\left(75 \mathrm{MHz}, \mathrm{CDCl}_{3}\right): \quad \delta[\mathrm{ppm}]=148.21\left(2 \mathrm{C}, \mathrm{C}_{4 \mathrm{a}, 10 \mathrm{a}}\right), 130.13\left(2 \mathrm{C}, \mathrm{C}_{3,6}\right), 130.06\left(2 \mathrm{C}, \mathrm{C}_{4,5}\right)$, $125.64\left(2 \mathrm{C}, \mathrm{C}_{2,7}\right), 124.70\left(2 \mathrm{C}, \mathrm{C}_{1,8}\right), 13.85\left(1 \mathrm{C}, \mathrm{CH}_{3}\right)$.

EI MS

$m / z(\%): \quad 193(100)[\mathrm{M}]^{+}$.

\subsubsection{Synthesis of 9-(bromomethylene)acridine (5)}

4 (0.84 g, $4.35 \mathrm{mmol}, 1.0 \mathrm{eq}), N$-bromosuccinimide $(0.83 \mathrm{~g}, 4.66 \mathrm{mmol}, 1.1 \mathrm{eq})$, and benzoic peroxide ( $72 \mathrm{mg}, 301 \mu \mathrm{mol}, 7 \mathrm{~mol} \%$ ) were dissolved in chloroform and heated to $65{ }^{\circ} \mathrm{C}$ for $3 \mathrm{~h}$. When the reaction mixture was cooled to room temperature, a recrystallisation from ethanol was applied. A pure, yellow product was obtained $(0.65 \mathrm{~g}, 2.40 \mathrm{mmol}, 55.2 \%)$ after a column chromatographic work-up (PE/EA, 5:1).

Chemical formula: $\quad \mathrm{C}_{14} \mathrm{H}_{10} \mathrm{BrN}$

Molecular weight: $\quad 272.15 \mathrm{~g} / \mathrm{mol}$<smiles>BrCc1c2ccccc2nc2ccccc12</smiles>

\section{${ }^{1}$ H NMR}

$\left(300 \mathrm{MHz}, \mathrm{CDCl}_{3}\right): \quad \delta[\mathrm{ppm}]=8.34\left(\mathrm{~d},{ }^{3} J=8.7 \mathrm{~Hz}, 2 \mathrm{H}, \mathrm{H}_{4,5}\right), 8.27\left(\mathrm{~d},{ }^{3} J=8.7 \mathrm{~Hz}, 2 \mathrm{H}, \mathrm{H}_{1,8}\right)$, 7.82 (ddd, ${ }^{3} J=8.7 \mathrm{~Hz}, 6.6 \mathrm{~Hz},{ }^{4} J=1.2 \mathrm{~Hz}, 2 \mathrm{H}, \mathrm{H}_{3,6}$ ), 7.69 (ddd, $\left.{ }^{3} J=8.7 \mathrm{~Hz}, 6.6 \mathrm{~Hz},{ }^{4} J=1.2 \mathrm{~Hz}, 2 \mathrm{H}, \mathrm{H}_{2,7}\right), 5.41\left(\mathrm{~s}, 2 \mathrm{H}, \mathrm{C}_{2}\right)$.

${ }^{13}$ C NMR

$\left(75 \mathrm{MHz}, \mathrm{CDCl}_{3}\right): \quad \delta[\mathrm{ppm}]=147.93\left(2 \mathrm{C}, \mathrm{C}_{4 \mathrm{a}, 10 \mathrm{a}}\right), 140.72\left(1 \mathrm{C}, \mathrm{C}_{9}\right), 131.07\left(2 \mathrm{C}, \mathrm{C}_{3,6}\right)$, $129.64\left(2 \mathrm{C}, \mathrm{C}_{4,5}\right), 127.22\left(2 \mathrm{C}, \mathrm{C}_{2,7}\right), 124.01\left(2 \mathrm{C}, \mathrm{C}_{8 \mathrm{a}, 9 \mathrm{a}}\right), 123.63(2 \mathrm{C}$, $\left.\mathrm{C}_{1,8}\right), 22.96\left(1 \mathrm{C}, \underline{\mathrm{C}} \mathrm{H}_{2}\right)$.

\section{EI MS}

$\mathrm{m} / \mathrm{z}(\%)$ :

$271(10)[\mathrm{M}]^{+\bullet}, 192(100)[\mathrm{M}-\mathrm{Br}]^{+\bullet}$. 


\subsubsection{Synthesis of 2-bis( $p$-nitrobenzenesulfonyl)aminodiphenylamine (6)}

A suspension of $p$-nitrobenzenesulfonyl chloride $(1.80 \mathrm{~g}, 8.12 \mathrm{mmol}, 3.0 \mathrm{eq})$ in DCM $(5 \mathrm{ml})$ was given to a solution of 2-aminodiphenylamine $(0.50 \mathrm{~g}, 2.69 \mathrm{mmol}, 1.0 \mathrm{eq})$ and trimethylamine $(1.15 \mathrm{ml}, 8.30 \mathrm{mmol}, 3.1 \mathrm{eq})$ in DCM $(20 \mathrm{ml})$. After $1 \mathrm{~h}$ at room temperature, the solvent was removed under reduced pressure and the obtained solid was treated with $\mathrm{CHCl}_{3}(7 \mathrm{ml})$. The resulting suspension was heated, filtrated and the residue was washed with DCM. The target compound (1.11 g, $2.00 \mathrm{mmol}, 74.3 \%)$ was obtained as orange powder.

Chemical formula: $\quad \mathrm{C}_{24} \mathrm{H}_{18} \mathrm{~N}_{4} \mathrm{O}_{8} \mathrm{~S}_{2}$

Molecular weight: $\quad 554.55 \mathrm{~g} / \mathrm{mol}$

${ }^{1}$ H NMR

$\left(300 \mathrm{MHz}, \mathrm{DMSO}-d_{6}\right): \quad \delta[\mathrm{ppm}]=8.35\left(\mathrm{~d},{ }^{3} J=8.9 \mathrm{~Hz}, 4 \mathrm{H}\right), 8.14\left(\mathrm{~d},{ }^{3} J=9.1 \mathrm{~Hz}, 4 \mathrm{H}\right), 7.43$ $\left(\mathrm{ddd},{ }^{3} J=8.5 \mathrm{~Hz}, 7.0 \mathrm{~Hz},{ }^{4} J=1.7 \mathrm{~Hz}, 1 \mathrm{H}\right), 7.29\left(\mathrm{dd},{ }^{3} J=8.3 \mathrm{~Hz}\right.$, $\left.{ }^{4} J=1.4 \mathrm{~Hz}, 1 \mathrm{H}\right), 7.12\left(\mathrm{t},{ }^{3} J=7.9 \mathrm{~Hz}, 2 \mathrm{H}\right), 6.99\left(\mathrm{ddd},{ }^{3} J=8.5 \mathrm{~Hz}\right.$, $\left.7.1 \mathrm{~Hz},{ }^{4} \mathrm{~J}=1.5 \mathrm{~Hz}, 1 \mathrm{H}\right), 6.91\left(\mathrm{dd},{ }^{3} \mathrm{~J}=7.9 \mathrm{~Hz},{ }^{4} J=1.7 \mathrm{~Hz}, 1 \mathrm{H}\right)$, 6.84-6.74 (m, $3 \mathrm{H}), 6.42$ (s, $1 \mathrm{H}, \mathrm{NH})$.

\section{EI MS}

m/z (\%): $\left.\quad 554(16)[\mathrm{M}]^{+\bullet}, 368(20)[\mathrm{M}-(\mathrm{Nos})]^{+\bullet}, 181(100)[\mathrm{M}-2(\mathrm{Nos}) \mathrm{H})\right]^{+\bullet}$.

\subsubsection{Synthesis of 2-nitrodiphenylamine (7)}

2-Nitroaniline $(0.55 \mathrm{~g}, 4.00 \mathrm{mmol}, 1.0 \mathrm{eq}), \mathrm{K}_{2} \mathrm{CO}_{3}(1.11 \mathrm{~g}, 8.00 \mathrm{mmol}, 2.0 \mathrm{eq})$, CuI $(76 \mathrm{mg}$, $0.40 \mathrm{mmol}, 10 \mathrm{~mol} \%$ ), and $L$-proline ( $92 \mathrm{mg}, 0.80 \mathrm{mmol}, 20 \mathrm{~mol} \%$ ) were treated with an excess of bromobenzene $(7 \mathrm{ml})$ which was used as reagent and solvent. Due to the poor solubility of the starting materials, DMSO was added $(3 \mathrm{ml})$. The mixture was heated to $125^{\circ} \mathrm{C}$ for $48 \mathrm{~h}$, then the solvent was removed under reduced pressure at $40{ }^{\circ} \mathrm{C}$. Brine was added and the solution was extracted three times with ethyl acetate. The gathered organic layers were washed two times with brine. The organic phase was dried over $\mathrm{MgSO}_{4}$, filtrated and the solvent was again removed under reduced pressure. The crude product was purified by column chromatography (PE/EA, 40:1) to give $7(0.57 \mathrm{~g}, 2.66 \mathrm{mmol}, 66.5 \%)$ as a yellow powder.

Chemical formula: $\quad \mathrm{C}_{12} \mathrm{H}_{10} \mathrm{~N}_{4} \mathrm{O}_{4}$

Molecular weight: $\quad 274.24 \mathrm{~g} / \mathrm{mol}$

DC $\left(\mathrm{SiO}_{2}\right): \quad \quad R_{\mathrm{f}}=0.38(\mathrm{PE} / \mathrm{EA}, 20: 1)$<smiles>O=[N+]([O-])c1ccccc1Nc1ccccc1</smiles> 


\section{${ }^{1}$ H NMR}

$\left(300 \mathrm{MHz}, \mathrm{CDCl}_{3}\right): \quad \delta[\mathrm{ppm}]=9.49(\mathrm{~s}, 1 \mathrm{H}, \mathrm{NH}), 8.21\left(\mathrm{dd},{ }^{3} \mathrm{~J}=8.5 \mathrm{~Hz},{ }^{4} J=1.3 \mathrm{~Hz}, 1 \mathrm{H}, \mathrm{H}_{3}\right)$, 7.47-7.35 (m, $\left.2 \mathrm{H}, \mathrm{H}_{\mathrm{Ph}}\right), 7.38-7.33\left(\mathrm{~m}, 1 \mathrm{H}, \mathrm{H}_{5}\right), 7.28\left(\mathrm{~d},{ }^{3} J=8.2 \mathrm{~Hz}, 2 \mathrm{H}\right.$, $\left.\mathrm{H}_{\mathrm{Ph}}\right), 7.23\left(\mathrm{~d},{ }^{3} J=7.6 \mathrm{~Hz}, 2 \mathrm{H}, \mathrm{H}_{6}\right), 6.77\left(\mathrm{ddd},{ }^{3} J=8.5 \mathrm{~Hz},{ }^{3} J=7.0 \mathrm{~Hz}\right.$, $\left.{ }^{4} J=1.3 \mathrm{~Hz}, 1 \mathrm{H}, \mathrm{H}_{4}\right)$.

\section{${ }^{13}$ C NMR}

$\left(75 \mathrm{MHz}, \mathrm{CDCl}_{3}\right): \quad \delta[\mathrm{ppm}]=143.20\left(1 \mathrm{C}, \mathrm{C}_{1}\right), 138.85\left(1 \mathrm{C}, \mathrm{C}_{7}\right), 135.77\left(1 \mathrm{C}, \mathrm{C}_{5}\right), 133.36$

$\left(1 \mathrm{C}, \mathrm{C}_{2}\right), 129.85\left(2 \mathrm{C}, \mathrm{C}_{\mathrm{Ph}}\right), 126.78\left(1 \mathrm{C}, \mathrm{C}_{3}\right), 125.77\left(1 \mathrm{C}, \mathrm{C}_{\mathrm{Ph}}\right), 124.50$

$\left(2 \mathrm{C}, \mathrm{C}_{\mathrm{Ph}}\right), 117.61\left(1 \mathrm{C}, \mathrm{C}_{4}\right), 116.17\left(1 \mathrm{C}, \mathrm{C}_{6}\right)$.

\section{EI MS}

$m / z(\%)$ :

$214.1(100)[\mathrm{M}]^{+\bullet}, 197.2(12)[\mathrm{M}-\mathrm{O}]^{+\bullet}, 180.2(22)\left[\mathrm{M}-\mathrm{HO}_{2}\right]^{+\bullet}, 167.2(64)$

$\left[\mathrm{M}-\mathrm{HNO}_{2}\right]^{+\cdot}$.

\subsubsection{Synthesis of 4,5-bis(bromomethylene)acridine (8)}

A solution of acridine ( $4.06 \mathrm{~g}, 22.6 \mathrm{mmol}, 1.0 \mathrm{eq})$ in conc. $\mathrm{H}_{2} \mathrm{SO}_{4}(50 \mathrm{ml})$ was heated up to $50{ }^{\circ} \mathrm{C}$ when BMME (7.4 ml, $90.6 \mathrm{mmol}, 4.0 \mathrm{eq})$ was added. After stirring for $17 \mathrm{~h}$ at this temperature, the reaction mixture was cooled to room temperature and the formed excess of bromine gas was discharged through an aqueous solution of thiosulfate. The crude product was crushed out by pouring onto ice $(400 \mathrm{~g})$ and stirring for $1 \mathrm{~h}$. The yellow precipitate was filtered, washed several times with dem. water and dissolved with chloroform $(400 \mathrm{ml})$. The solution was washed with brine (3x $200 \mathrm{ml})$ and the gathered aqueous phases were extracted with chloroform (100 $\mathrm{ml})$. The organic phases were dried over $\mathrm{MgSO}_{4}$, filtered and the solvent was removed under reduced pressure. For purification, the crude dark yellow product was recrystallised from chloroform two times to obtain a light yellow powder (2.54 g, $6.96 \mathrm{mmol}, 30.7 \%)$.

\footnotetext{
Chemical formula: $\quad \mathrm{C}_{15} \mathrm{H}_{11} \mathrm{Br}_{2} \mathrm{~N}$

Molecular weight: $\quad 365.06 \mathrm{~g} / \mathrm{mol}$

DC $\left(\mathrm{SiO}_{2}\right): \quad R_{\mathrm{f}}=0.62(\mathrm{MeOH})$<smiles>BrCc1cccc2cc3cccc(CBr)c3nc12</smiles>

${ }^{1}$ H NMR

$\left(300 \mathrm{MHz}, \mathrm{CDCl}_{3}\right): \quad \delta[\mathrm{ppm}]=8.78\left(\mathrm{~s}, 1 \mathrm{H}, \mathrm{H}_{9}\right), 7.99\left(\mathrm{~d},{ }^{3} J=8.5 \mathrm{~Hz}, 2 \mathrm{H}, \mathrm{H}_{1,8}\right), 7.94(\mathrm{~d}$, $\left.{ }^{3} J=6.8 \mathrm{~Hz}, 2 \mathrm{H}, \mathrm{H}_{3,6}\right), 7.51\left(\mathrm{dd},{ }^{3} J=8.5 \mathrm{~Hz}, 6.9 \mathrm{~Hz}, 2 \mathrm{H}, \mathrm{H}_{2,7}\right), 5.43$ (s, $4 \mathrm{H}, \mathrm{CH}_{2}$ ).
}

\section{${ }^{13}$ C NMR}

$\left(75 \mathrm{MHz}, \mathrm{CDCl}_{3}\right): \quad \delta[\mathrm{ppm}]=145.81\left(2 \mathrm{C}, \mathrm{C}_{4 \mathrm{a}, 10 \mathrm{a}}\right), 136.75\left(1 \mathrm{C}, \mathrm{C}_{9}\right), 136.54\left(2 \mathrm{C}, \mathrm{C}_{4,5}\right)$, $131.34\left(2 \mathrm{C}, \mathrm{C}_{3,6}\right), 129.18\left(2 \mathrm{C}, \mathrm{C}_{1,8}\right), 126.93\left(2 \mathrm{C}, \mathrm{C}_{8 \mathrm{a}, 9 \mathrm{a}}\right), 125.99(2 \mathrm{C}$, $\left.\mathrm{C}_{2,7}\right), 30.27\left(2 \mathrm{C}, \mathrm{CH}_{2}\right)$. 


\section{EI MS}

$\mathrm{m} / \mathrm{z}(\%)$ :

$365(34)[\mathrm{M}]^{+\bullet}, 286 / 284(76 / 76)[\mathrm{M}-\mathrm{Br}]^{+\bullet}, 205(100)[\mathrm{M}-2 \mathrm{Br}]^{+\bullet}$.

Elemental analysis

in $\%$ (calculated):

C: 48.86 (49.35), H: 3.06 (3.04), N: 3.78 (3.84), Br: 43.30 (43.78).

\subsubsection{Synthesis of 4,5-bis(bromomethylene)-9-methylacridine (9)}

The synthesis of $\mathbf{9}$ is the same as for $\mathbf{8}$, with $4(5.0 \mathrm{~g}, 25.88 \mathrm{mmol}, 1.0 \mathrm{eq})$, BMME $(9.3 \mathrm{ml}$, $114.0 \mathrm{mmol}, 4.4 \mathrm{eq})$ and conc. $\mathrm{H}_{2} \mathrm{SO}_{4}(70 \mathrm{ml})$. The crude product was purified by column chromatography (PE/EA, 10:1) which resulted in a yellow powder $(5.28 \mathrm{~g}, 13.96 \mathrm{mmol}, 53.9 \%)$. The crystallisation of 9 was performed in DCM at $-30{ }^{\circ} \mathrm{C}$.

Chemical formula: $\quad \mathrm{C}_{16} \mathrm{H}_{13} \mathrm{Br}_{2} \mathrm{~N}$

Molecular weight: $\quad 379.09 \mathrm{~g} / \mathrm{mol}$

$\mathrm{DC}\left(\mathrm{SiO}_{2}\right): \quad R_{\mathrm{f}}=0.11(\mathrm{PE} / \mathrm{EA}, 10: 1)$<smiles>Cc1c2ccccc2c(CBr)c2nc3c(CBr)cccc3c(CBr)c12</smiles>

${ }^{1}$ H NMR

$\left(300 \mathrm{MHz}, \mathrm{CDCl}_{3}\right): \quad \delta[\mathrm{ppm}]=8.22\left(\mathrm{dd},{ }^{3} J=8.9 \mathrm{~Hz},{ }^{4} J=1.1 \mathrm{~Hz}, 2 \mathrm{H}, \mathrm{H}_{1,8}\right), 7.91\left(\mathrm{dd},{ }^{3} J=6.8\right.$ $\left.\mathrm{Hz},{ }^{4} \mathrm{~J}=1.1 \mathrm{~Hz}, 2 \mathrm{H}, \mathrm{H}_{3,6}\right), 7.51\left(\mathrm{dd},{ }^{3} \mathrm{~J}=8.9 \mathrm{~Hz}, 6.8 \mathrm{~Hz}, 2 \mathrm{H}, \mathrm{H}_{2,7}\right), 5.44$ (s, $\left.4 \mathrm{H}, \underline{\mathrm{C}}_{2}\right), 3.07$ (s, $\left.3 \mathrm{H}, \mathrm{C}_{3}\right)$

${ }^{13}$ C NMR

$\left(75 \mathrm{MHz}, \mathrm{CDCl}_{3}\right): \quad \delta[\mathrm{ppm}]=145.15\left(2 \mathrm{C}, \mathrm{C}_{4 \mathrm{a}, 10 \mathrm{a}}\right), 143.01\left(1 \mathrm{C}, \mathrm{C}_{9}\right), 137.15\left(2 \mathrm{C}, \mathrm{C}_{4,5}\right)$, $130.75\left(2 \mathrm{C}, \mathrm{C}_{3,6}\right), 125.80\left(2 \mathrm{C}, \mathrm{C}_{1,8}\right), 125.62\left(2 \mathrm{C}, \mathrm{C}_{8 \mathrm{a}, 9 \mathrm{a}}\right), 125.55(2 \mathrm{C}$, $\left.\mathrm{C}_{2,7}\right), 30.80\left(2 \mathrm{C}, \mathrm{CH}_{2}\right), 14.10\left(1 \mathrm{C}, \mathrm{CH}_{3}\right)$.

\section{EI MS}

$m / z(\%): \quad 379(45)[\mathrm{M}]^{+\bullet}, 298(85)[\mathrm{M}-\mathrm{Br}]^{+\bullet}, 219(100)[\mathrm{M}-2 \mathrm{Br}]^{+\bullet}$.

\subsubsection{Synthesis of 4,5-bis(di(2'-methylthiobenzyl)aminemethylene)-9-methyl- acridine (10)}

9 (0.54 g, $1.43 \mathrm{mmol}, 1.0 \mathrm{eq})$, bis(2-methylthiobenzyl)amine (0.82 g, $2.85 \mathrm{mmol}, 2.0 \mathrm{eq})$ and $\mathrm{K}_{2} \mathrm{CO}_{3}(0.79 \mathrm{~g}, 5.72 \mathrm{mmol}, 4.0 \mathrm{eq})$ were dissolved in $\mathrm{MeCN}(22 \mathrm{ml})$ and heated up to $85^{\circ} \mathrm{C}$ for $3 \mathrm{~h}$. After cooling to room temperature, the solvent was decanted and the crude product was washed with hexane and dissolved again in toluene. The insoluble inorganic salts were filtered off after what the solvent was removed under reduced pressure. The desired compound $(0.54 \mathrm{~g}$, $0.68 \mathrm{mmol}, 47.6 \%$ ) could be obtained as a bright yellow powder. Suitable crystals for X-ray analysis were obtained after recrystallisation from a pentane/ethyl acetate mixture. 
Chemical formula: $\quad \mathrm{C}_{48} \mathrm{H}_{49} \mathrm{~N}_{3} \mathrm{~S}_{4}$

Molecular weight: $\quad 795.28 \mathrm{~g} / \mathrm{mol}$

DC $\left(\mathrm{SiO}_{2}\right): \quad R_{\mathrm{f}}=0.2(\mathrm{PE} / \mathrm{EA}, 20: 1)$

${ }^{1}$ H NMR

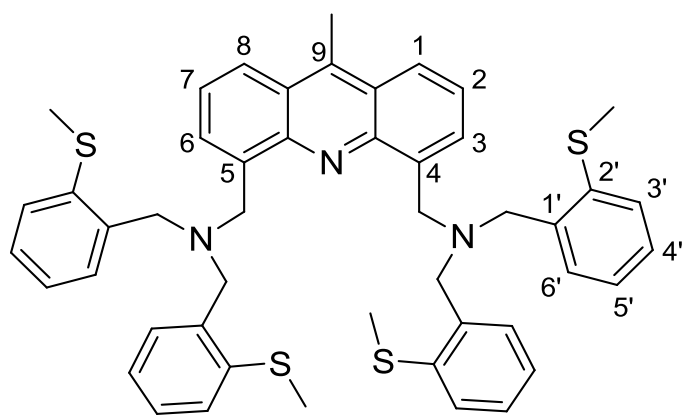

$\left(300 \mathrm{MHz}, \mathrm{CDCl}_{3}\right): \quad \delta[\mathrm{ppm}]=8.25\left(\mathrm{~d},{ }^{3} J=7.7 \mathrm{~Hz}, 2 \mathrm{H}, \mathrm{H}_{1,8}\right), 7.98\left(\mathrm{~d},{ }^{3} J=6.5 \mathrm{~Hz}, 2 \mathrm{H}, \mathrm{H}_{3,6}\right)$, 7.67-7.61 (m, $\left.4 \mathrm{H}, \mathrm{H}_{6}\right)$, 7.63-7.56 (m, $\left.2 \mathrm{H}, \mathrm{H}_{2,7}\right), 7.27-7.17(\mathrm{~m}, 8 \mathrm{H}$,

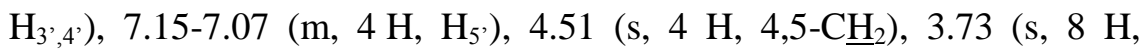
$\left.\mathrm{NC}_{2}\right), 3.08$ (s, $\left.3 \mathrm{H}, 9-\underline{\mathrm{C}}_{3}\right), 2.40$ (s, $\left.12 \mathrm{H}, \mathrm{SC}_{3}\right)$.

${ }^{13}$ C NMR

$\left(75 \mathrm{MHz}, \mathrm{CDCl}_{3}\right): \quad \delta[\mathrm{ppm}]=145.60\left(2 \mathrm{C}, \mathrm{C}_{4 \mathrm{a}, 10 \mathrm{a}}\right), 137.41\left(4 \mathrm{C}, \mathrm{C}_{2}\right), 136.89\left(2 \mathrm{C}, \mathrm{C}_{4,5}\right)$, $136.46\left(4 \mathrm{C}, \mathrm{C}_{1}\right), 128.69\left(2 \mathrm{C}, \mathrm{C}_{3,6}\right), 128.04\left(4 \mathrm{C}, \mathrm{C}_{6}\right), 127.38\left(4 \mathrm{C}, \mathrm{C}_{3}\right)$ ), 125.30 ( $\left.2 \mathrm{C}, \mathrm{C}_{2,7}\right), 124.94\left(4 \mathrm{C}, \mathrm{C}_{4}\right), 124.59$ (2 C, $\mathrm{C}_{8 \mathrm{a}, 9 \mathrm{a}}$ ), 124.48 (4 C, $\left.\mathrm{C}_{5}\right), 123.67\left(2 \mathrm{C}, \mathrm{C}_{1,8}\right), 55.27\left(4 \mathrm{C}, \mathrm{NCH}_{2}\right), 52.68\left(2 \mathrm{C}, 4,5-\underline{\mathrm{CH}}_{2}\right), 14.91$ $\left(4 \mathrm{C}, \mathrm{SCH}_{3}\right), 13.74\left(1 \mathrm{C}, 9-\underline{\mathrm{CH}}_{3}\right)$.

EI MS

$m / z(\%)$ :

$795.2(100)[\mathrm{M}]^{+\bullet}, 780.2(24)[\mathrm{M}-\mathrm{Me}]^{+*}$

\subsubsection{Synthesis of $4,5-\operatorname{bis}\left(N, N^{\prime}, N^{\prime}\right.$-trimethylethylenediaminemethylene)acridine} (11)

$\mathrm{K}_{2} \mathrm{CO}_{3}(1.35 \mathrm{~g}, 13.23 \mathrm{mmol}, 3.5 \mathrm{eq})$ and $8(1.38 \mathrm{~g}, 3.78 \mathrm{mmol}, 1.0 \mathrm{eq})$ formed a yellow suspension with $\mathrm{MeCN}(60 \mathrm{ml})$. When $N, N^{\prime}, N^{\prime}$-trimethylethylenediamine $(1.00 \mathrm{ml}, 7.75 \mathrm{mmol}$, $2.1 \mathrm{eq)} \mathrm{was} \mathrm{added,} \mathrm{the} \mathrm{temperature} \mathrm{was} \mathrm{increased} \mathrm{to} 85{ }^{\circ} \mathrm{C}$ and the reaction mixture was stirred for $8 \mathrm{~h}$. The volatile compounds were removed under reduced pressure and ethyl acetate and brine was added to the crude product. The organic phase was separated and washed with brine. The aqueous phases were extracted five times with ethyl acetate and all gathered organic layers were again washed with brine. The solvent was dried over $\mathrm{MgSO}_{4}$, filtered and removed in vacuo. The target compound was obtained as brown oil (212 mg, $520 \mu \mathrm{mol}, 13.8 \%)$.

Chemical formula: $\quad \mathrm{C}_{25} \mathrm{H}_{37} \mathrm{~N}_{5}$

Molecular weight: $\quad 407.61 \mathrm{~g} / \mathrm{mol}$

DC $\left(\mathrm{Al}_{\mathbf{2}} \mathbf{O}_{3}\right): \quad \quad R_{\mathrm{f}}=0.38(\mathrm{EA} / \mathrm{MeOH}, 2: 1)$.

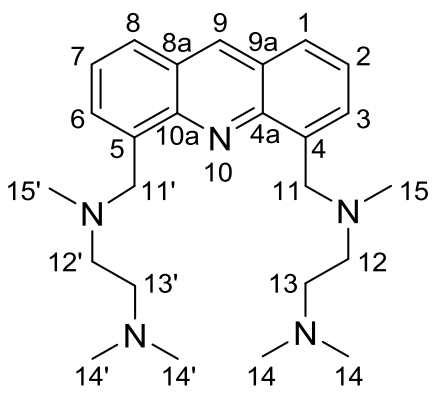




\section{${ }^{1}$ H NMR}

$\left(300 \mathrm{MHz}, \mathrm{CDCl}_{3}\right): \quad \delta[\mathrm{ppm}]=8.69\left(\mathrm{~s}, 1 \mathrm{H}, \mathrm{H}_{9}\right), 7.92-7.82\left(\mathrm{~m}, 4 \mathrm{H}, \mathrm{H}_{1,3,6,8}\right), 7.50\left(\mathrm{dd},{ }^{3} J=\right.$ $\left.8.4 \mathrm{~Hz}, 6.9 \mathrm{~Hz}, 2 \mathrm{H}, \mathrm{H}_{2,7}\right), 4.46\left(\mathrm{~s}, 4 \mathrm{H}, \mathrm{H}_{11,11}\right), 2.82-8.73(\mathrm{~m}, 4 \mathrm{H}$, $\left.\mathrm{H}_{12,12^{\prime}}\right), 2.68-2.59\left(\mathrm{~m}, 4 \mathrm{H}, \mathrm{H}_{13,13}\right), 2.42\left(\mathrm{~s}, 6 \mathrm{H}, \mathrm{H}_{15,15}\right), 2.30$ (s, $12 \mathrm{H}$, $\mathrm{H}_{14, \mathrm{H} 14) \text {. }}$

\section{${ }^{13}$ C NMR}

$\left(75 \mathrm{MHz}, \mathrm{CDCl}_{3}\right): \quad \delta[\mathrm{ppm}]=147.09\left(2 \mathrm{C}, \mathrm{C}_{4 \mathrm{a}, 10 \mathrm{a}}\right), 136.97\left(2 \mathrm{C}, \mathrm{C}_{4,5}\right), 136.13\left(1 \mathrm{C}, \mathrm{C}_{9}\right)$, $129.72\left(2 \mathrm{C}, \mathrm{C}_{3,6}\right), 127.05\left(2 \mathrm{C}, \mathrm{C}_{1,8}\right), 126.37\left(2 \mathrm{C}, \mathrm{C}_{8 \mathrm{a}, 9 \mathrm{a}}\right), 125.49(2 \mathrm{C}$, $\left.\mathrm{C}_{2,7}\right), 57.19\left(2 \mathrm{C}, \mathrm{C}_{11,11^{\prime}}\right), 57.03\left(2 \mathrm{C}, \mathrm{C}_{13,13}\right), 55.44\left(2 \mathrm{C}, \mathrm{C}_{12,12}\right), 45.34$ (4 C, $\left.\mathrm{C}_{14,14}\right), 43.03\left(2 \mathrm{C}, \mathrm{C}_{15,15^{\prime}}\right)$.

\subsubsection{Synthesis of 4,5-bis( $N, N^{\prime}, N^{\prime}$-trimethylethylenediaminemethylene)-9-methyl- acridine (12)}

The formation of the methyl derivative $9(396 \mathrm{mg}, 1.04 \mathrm{mmol}, 1.0 \mathrm{eq})$ with $N, N^{\prime}, N^{\prime}-$ trimethylethylenediamine $(0.29 \mathrm{ml}, 2.23 \mathrm{mmol}, 2.1 \mathrm{eq})$ and $\mathrm{K}_{2} \mathrm{CO}_{3}(0.55 \mathrm{~g}, 3.98 \mathrm{mmol}, 3.8 \mathrm{eq})$ was performed in $\mathrm{MeCN}(40 \mathrm{ml})$ at $50{ }^{\circ} \mathrm{C}$. The reaction mixture was stirred for 16 hours and afterwards purified by column chromatography on alumina (EA/MeOH, 1:0 $\rightarrow$ EA/MeOH, 20:1). 12 (75 mg, $178 \mu \mathrm{mol}, 17.1 \%$ ) could be obtained as a yellow solid.

Chemical formula: $\quad \mathrm{C}_{26} \mathrm{H}_{39} \mathrm{~N}_{5}$

Molecular weight: $\quad 421.32 \mathrm{~g} / \mathrm{mol}$

DC $\left(\mathbf{A l}_{\mathbf{2}} \mathbf{O}_{3}\right): \quad R_{\mathrm{f}}=0.17(\mathrm{EA} / \mathrm{MeOH}, 10: 1)$

${ }^{1}$ H NMR

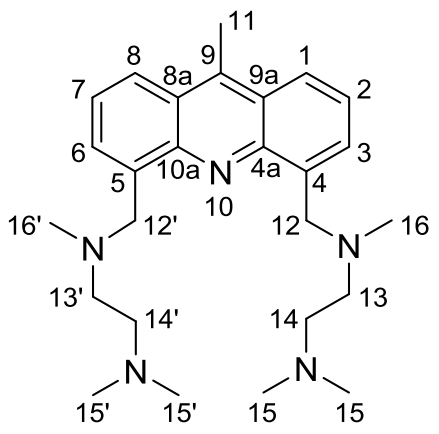

$\left(300 \mathrm{MHz}, \mathrm{CDCl}_{3}\right): \quad \delta[\mathrm{ppm}]=8.11\left(\mathrm{~d},{ }^{3} J=8.6 \mathrm{~Hz}, 2 \mathrm{H}, \mathrm{H}_{1,8}\right), 7.87\left(\mathrm{~d},{ }^{3} J=6.7 \mathrm{~Hz}, 2 \mathrm{H}, \mathrm{H}_{3,6}\right)$, $7.50\left(\mathrm{dd},{ }^{3} \mathrm{~J}=8.6 \mathrm{~Hz}, 6.7 \mathrm{~Hz}, 2 \mathrm{H}, \mathrm{H}_{2,7}\right), 4.46\left(\mathrm{~s}, 4 \mathrm{H}, \mathrm{H}_{12}, 12^{2}\right), 3.07$ (s, $\left.3 \mathrm{H}, \mathrm{H}_{11}\right), 2.72\left(\mathrm{dd},{ }^{3} \mathrm{~J}=8.4 \mathrm{~Hz}, 5.7 \mathrm{~Hz}, 4 \mathrm{H}, \mathrm{H}_{13,13}\right), 2.53\left(\mathrm{dd},{ }^{3} \mathrm{~J}=\right.$ $\left.8.4 \mathrm{~Hz}, 5.7 \mathrm{~Hz}, 4 \mathrm{H}, \mathrm{H}_{14,14}\right), 2.24\left(\mathrm{~s}, 6 \mathrm{H}, \mathrm{H}_{16,16}\right), 2.17$ (s, $12 \mathrm{H}, \mathrm{H}_{15,15}$ ).

${ }^{13}$ C NMR

$\left(75 \mathrm{MHz}, \mathrm{CDCl}_{3}\right): \quad \delta[\mathrm{ppm}]=146.37\left(2 \mathrm{C}, \mathrm{C}_{4 \mathrm{a}}, 10 \mathrm{a}\right), 141.84\left(1 \mathrm{C}, \mathrm{C}_{9}\right), 138.03\left(2 \mathrm{C}, \mathrm{C}_{4,5}\right)$, $129.03\left(2 \mathrm{C}, \mathrm{C}_{3,6}\right), 125.23\left(2 \mathrm{C}, \mathrm{C}_{1,8}\right), 125.17\left(2 \mathrm{C}, \mathrm{C}_{8 \mathrm{a}, 9 \mathrm{a}}\right), 123.22(2 \mathrm{C}$, $\left.\mathrm{C}_{2,7}\right), 57.85\left(2 \mathrm{C}, \mathrm{C}_{12,12^{2}}\right), 57.62\left(2 \mathrm{C}, \mathrm{C}_{14,14}\right), 56.24\left(2 \mathrm{C}, \mathrm{C}_{13,13^{\prime}}\right), 46.05$ (4 C, $\left.\mathrm{C}_{15,15^{\prime}}\right), 43.27\left(2 \mathrm{C}, \mathrm{C}_{16,16}\right), 14.00\left(1 \mathrm{C}, \mathrm{C}_{11}\right)$.

\section{EI MS}

$\mathrm{m} / \mathrm{z}(\%)$ :

$421 \quad(10) \quad[\mathrm{M}]^{+\bullet}, \quad 363 \quad(65) \quad\left[\mathrm{M}-\left(\mathrm{NMe}_{2}\right)_{2}\right]^{+*}, \quad 319 \quad$ (30) $\quad[\mathrm{M}-$ $\left.\left(\mathrm{NMeC}_{2} \mathrm{H}_{4} \mathrm{NMe}_{2}\right)\right]^{+\bullet}, 220(100)\left[\mathrm{M}-\left(\mathrm{NMeC}_{2} \mathrm{H}_{4} \mathrm{NMe}_{2}\right)_{2}\right]^{+\bullet}$. 


\subsubsection{Synthesis of 4,5-bis( $N$-methyl- $N$-phenylaminemethylene)acridine (13)}

To a suspension of $8(0.50 \mathrm{~g}, 1.37 \mathrm{mmol}, 1.0 \mathrm{eq})$ and $\mathrm{K}_{2} \mathrm{CO}_{3}(0.66 \mathrm{~g}, 4.78 \mathrm{mmol}, 3.5 \mathrm{eq})$ in $\mathrm{MeCN}$ $(30 \mathrm{ml})$, methylphenylamine $(0.30 \mathrm{ml}, 2.76 \mathrm{mmol}, 2.0 \mathrm{eq})$ was added. After stirring the reaction mixture for $4 \mathrm{~h}$ at room temperature, the volatile components were removed under reduced pressure. The crude product was dissolved in ethyl acetate and washed three times with brine. The aqueous phases were again extracted with ethyl acetate and the gathered organic layers were dried over $\mathrm{MgSO}_{4}$, filtrated and the solvent was removed in vacuo. For further purification, a recrystallisation from methanol was implemented. Thereby, the impurities were dissolved and filtrated off. 13 ( $0.49 \mathrm{mg}, 1.17 \mathrm{mmol}, 85.4 \%$ ) could be obtained as a yellow solid.

Chemical formula: $\quad \mathrm{C}_{29} \mathrm{H}_{27} \mathrm{~N}_{3}$

Molecular weight: $\quad 417.55 \mathrm{~g} / \mathrm{mol}$

\section{${ }^{1}$ H NMR}<smiles>CN(Cc1cccc2cc3cccc(CN(C)c4ccccc4)c3nc12)c1ccccc1</smiles>

(300 MHz, DMSO- $\left.d_{6}\right): \quad \delta[\mathrm{ppm}]=9.15\left(\mathrm{~s}, 1 \mathrm{H}, \mathrm{H}_{9}\right), 8.07\left(\mathrm{~d},{ }^{3} J=8.3 \mathrm{~Hz}, 2 \mathrm{H}, \mathrm{H}_{1,8}\right), 7.55$ (dd, $\left.{ }^{3} J=8.3 \mathrm{~Hz}, 6.9 \mathrm{~Hz}, 2 \mathrm{H}, \mathrm{H}_{2,7}\right), 7.47\left(\mathrm{~d},{ }^{3} J=6.9 \mathrm{~Hz}, 2 \mathrm{H}, \mathrm{H}_{3,6}\right), 7.12$ $\left(\mathrm{dd},{ }^{3} J=8.6 \mathrm{~Hz}, 7.2 \mathrm{~Hz}, 4 \mathrm{H}, \operatorname{meta} \mathrm{H}_{\mathrm{Ph}}\right), 6.73\left(\mathrm{~d},{ }^{3} J=8.6 \mathrm{~Hz}, 4 \mathrm{H}\right.$, ortho $\left.\mathrm{H}_{\mathrm{Ph}}\right), 6.58\left(\mathrm{t},{ }^{3} J=7.2 \mathrm{~Hz}, 2 \mathrm{H}\right.$, para $\left._{\mathrm{Ph}}\right), 5.38\left(\mathrm{~s}, 4 \mathrm{H}, \mathrm{CH}_{2}\right), 3.19$ (s, $6 \mathrm{H}, \mathrm{CH}_{3}$ ).

${ }^{13}$ C NMR

$\left(75 \mathrm{MHz}, \mathrm{DMSO}-d_{6}\right): \quad \delta[\mathrm{ppm}]=149.15\left(2 \mathrm{C}\right.$, ipso $\left.\mathrm{C}_{\mathrm{Ph}}\right), 145.76\left(2 \mathrm{C}, \mathrm{C}_{4 \mathrm{a}, 10 \mathrm{a}}\right), 136.73\left(1 \mathrm{C}, \mathrm{C}_{9}\right)$, $135.68\left(2 \mathrm{C}, \mathrm{C}_{4,5}\right), 128.98\left(4 \mathrm{C}, \operatorname{meta}_{\mathrm{Ph}}\right), 127.01\left(2 \mathrm{C}, \mathrm{C}_{1,8}\right), 126.79$ $\left(2 \mathrm{C}, \mathrm{C}_{3,6}\right), 126.10\left(2 \mathrm{C}, \mathrm{C}_{8 \mathrm{a}, 9 \mathrm{a}}\right), 125.64\left(2 \mathrm{C}, \mathrm{C}_{2,7}\right), 115.59(2 \mathrm{C}$, para $\left.\mathrm{C}_{\mathrm{Ph}}\right), 111.66\left(4 \mathrm{C}\right.$, ortho $\left.\mathrm{C}_{\mathrm{Ph}}\right), 52.55\left(2 \mathrm{C}, \mathrm{CH}_{2}\right), 38.87\left(2 \mathrm{C}, \mathrm{CH}_{3}\right)$.

\section{EI MS}

$\mathrm{m} / \mathrm{z}(\%)$ :

417.2 (14) $[\mathrm{M}]^{+\bullet}, 310.1(100) \quad[\mathrm{M}-\mathrm{NMePh}]^{+*}, 205.1$ (84) $\quad[\mathrm{M}-$ $\left.(\mathrm{NMePh})_{2}\right]^{+*}$.

Elemental analysis

in \% (calculated): $\quad$ C: 82.31 (83.42), H: $6.33(6.52), \mathrm{N}: 9.92(10.06)$.

\subsubsection{Synthesis of 4,5-bis $(N$-methyl- $N$-phenylaminemethylene)acridine hydro- chloride (14)}

13 (125 mg, $300 \mu \mathrm{mol}, 1.0 \mathrm{eq})$ was suspended in ethanol $(10 \mathrm{ml})$ and conc. aqueous $\mathrm{HCl}$ was added $(10 \mathrm{ml}, 120 \mathrm{mmol}, 400 \mathrm{eq})$. The reaction mixture was stirred at room temperature for $30 \mathrm{~min}$ whereby it became a clear solution. After adding dem. $\mathrm{H}_{2} \mathrm{O}(10 \mathrm{ml})$, the solution was extracted with hexane $(2 \times 20 \mathrm{ml})$. The organic phases were discarded and the volatile compounds 
were removed under reduced pressure. The product (106 mg, $216 \mu \mathrm{mol}, 72.0 \%)$ could be obtained as a yellow solid.

14 was dissolved in a boiling ethanol/hexane (1:1) mixture. Suitable crystals for X-ray analysis were obtained after storing the crystals for four weeks at room temperature.

Chemical formula: $\quad \mathrm{C}_{29} \mathrm{H}_{29} \mathrm{~N}_{3} \mathrm{Cl}_{2}$

Molecular weight: $\quad 490.47 \mathrm{~g} / \mathrm{mol}$

\section{${ }^{1}$ H NMR}

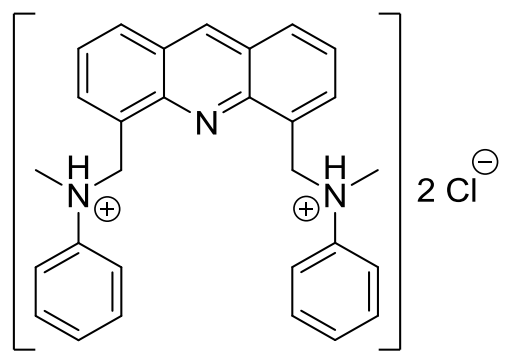

$\left(300 \mathrm{MHz}, \mathrm{DMSO}-d_{6}\right): \quad \delta[\mathrm{ppm}]=9.17\left(\mathrm{~s}, 1 \mathrm{H}, \mathrm{H}_{9}\right), 8.14\left(\mathrm{~d},{ }^{3} J=8.0 \mathrm{~Hz}, 2 \mathrm{H}, \mathrm{H}_{1,8}\right), 7.82-7.70$ $\left(\mathrm{m}, 2 \mathrm{H}, \mathrm{H}_{3,6}\right), 7.59\left(\mathrm{dd},{ }^{3} \mathrm{~J}=8.3 \mathrm{~Hz}, 6.9 \mathrm{~Hz}, 2 \mathrm{H}, \mathrm{H}_{2,7}\right), 7.29-7.08$ (m, $8 \mathrm{H}$, meta $_{\mathrm{Ph}}$, ortho $\left.\mathrm{H}_{\mathrm{Ph}}\right)$, 6.97-6.84 (m, $\left.2 \mathrm{H}, \operatorname{para}_{\mathrm{Ph}}\right), 5.48(\mathrm{~s}, 4 \mathrm{H}$, $\left.\mathrm{CH}_{2}\right), 3.28\left(\mathrm{~s}, 6 \mathrm{H}, \mathrm{CH}_{3}\right)$.

\section{EI MS}

$\mathrm{m} / \mathrm{z}(\%)$ :

$417.2(14)\left[\mathrm{M}-(\mathrm{HCl})_{2}\right]^{+\bullet}, 310.1(100)\left[\mathrm{M}-\mathrm{NMePh}(\mathrm{HCl})_{2}\right]^{+\bullet}, 205.1(84)$
$\left[\mathrm{M}-(\mathrm{NMePh})_{2}(\mathrm{HCl})_{2}\right]^{+\bullet}$

\section{Elemental analysis}

in \% (calculated):

14: C: 65.94 (71.02), H: 6.40 (5.96), N: 7.94 (8.57), Cl: 17.50 (14.46).

Chemical composition gained by X-ray analysis: $\mathbf{1 4}+\mathrm{H}_{3} \mathrm{O}^{+}+\mathrm{Cl}^{-}+$ EtOH: C: 65.94 (63.00), H: 6.40 (6.48), N: 7.94 (7.11), Cl: 17.50 (17.99).

\subsubsection{Synthesis of 4,5-bis(dimethylaminemethylene)acridine (15)}

The synthesis procedure for $\mathbf{1 5}$ is the same as for $\mathbf{1 3}$ with $\mathbf{8}(1.55 \mathrm{~g}, 4.25 \mathrm{mmol}, 1.0 \mathrm{eq}), \mathrm{K}_{2} \mathrm{CO}_{3}$ (1.76 g, $12.75 \mathrm{mmol}, 3.0 \mathrm{eq})$, dimethylamine in THF (2.0 M, $4.5 \mathrm{ml}, 9.0 \mathrm{mmol}, 2.1 \mathrm{eq})$ and $\mathrm{MeCN}$ $(40 \mathrm{ml})$. In this case, no recrystallisation was needed to obtain the product $(0.78 \mathrm{~g}, 2.66 \mathrm{mmol}$, $62.6 \%$ ) as a yellow-brown solid.

Chemical formula: $\quad \mathrm{C}_{19} \mathrm{H}_{23} \mathrm{~N}_{3}$

Molecular weight: $\quad 293.41 \mathrm{~g} / \mathrm{mol}$

DC $\left(\mathbf{A l}_{\mathbf{2}} \mathbf{O}_{3}\right): \quad R_{\mathrm{f}}=0.13(\mathrm{EA} / \mathrm{MeOH}, 10: 1)$.<smiles>CN(C)Cc1cccc2cc3cccc(CN(C)C)c3nc12</smiles>

${ }^{1}$ H NMR

$\left(300 \mathrm{MHz}, \mathrm{DMSO}-d_{6}\right): \quad \delta[\mathrm{ppm}]=9.09\left(\mathrm{~s}, 1 \mathrm{H}, \mathrm{H}_{9}\right), 8.08\left(\mathrm{dd},{ }^{3} \mathrm{~J}=8.5 \mathrm{~Hz},{ }^{4} \mathrm{~J}=1.6 \mathrm{~Hz}, 2 \mathrm{H}\right.$, $\left.\mathrm{H}_{1,8}\right), 7.86\left(\mathrm{dd},{ }^{3} J=6.8 \mathrm{~Hz},{ }^{4} J=1.3 \mathrm{~Hz}, 2 \mathrm{H}, \mathrm{H}_{3,6}\right), 7.61\left(\mathrm{dd},{ }^{3} J=8.5\right.$, 
$\left.6.8 \mathrm{~Hz}, 2 \mathrm{H}, \mathrm{H}_{2,7}\right), 4.28\left(\mathrm{~s}, 4 \mathrm{H}, \mathrm{CH}_{2}\right), 2.35\left(\mathrm{~s}, 12 \mathrm{H}, \mathrm{CH}_{3}\right)$.

${ }^{13}$ C NMR

$\left(75 \mathrm{MHz}, \mathrm{DMSO}-d_{6}\right): \quad \delta[\mathrm{ppm}]=146.22\left(2 \mathrm{C}, \mathrm{C}_{4 \mathrm{a}, 10 \mathrm{a}}\right), 136.60\left(2 \mathrm{C}, \mathrm{C}_{4,5}\right), 135.92\left(1 \mathrm{C}, \mathrm{C}_{9}\right)$, $129.96\left(2 \mathrm{C}, \mathrm{C}_{3,6}\right), 127.42\left(2 \mathrm{C}, \mathrm{C}_{1,8}\right), 125.89\left(2 \mathrm{C}, \mathrm{C}_{8 \mathrm{a}, 9 \mathrm{a}}\right), 125.55(2 \mathrm{C}$, $\left.\mathrm{C}_{2,7}\right), 58.24\left(2 \mathrm{C}, \mathrm{CH}_{2}\right), 45.40\left(4 \mathrm{C}, \mathrm{CH}_{3}\right)$.

${ }^{15}$ N NMR

$\left(40.6 \mathrm{MHz}, \mathrm{DMF}-d_{7}\right): \quad \delta[\mathrm{ppm}]=-81.2\left(\mathrm{~N}_{\text {arom }}\right),-355.3\left(\mathrm{NMe}_{2}\right)\left(\right.$ taken from $\left.{ }^{1} \mathrm{H},{ }^{15} \mathrm{~N}-\mathrm{HMBC}\right)$.

\section{EI MS}

$\mathrm{m} / \mathrm{z}(\%)$ :

293 (4) $[\mathrm{M}]^{+\bullet}, 250(64)\left[\mathrm{M}-\mathrm{NMe}_{2}\right]^{+\bullet}, 205(100)\left[\mathrm{M}-\left(\mathrm{NMe}_{2}\right)_{2}\right]^{+\bullet}$.

\section{Elemental analysis}

in \% (calculated): $\quad$ C: 76.45 (77.78), H: 7.77 (7.90), N: $13.94(14.32)$.

\subsubsection{Synthesis of 4,5-bis(dimethylaminemethylene)acridine hydrochloride (16)}

Conc. $\mathrm{HCl}(0.4 \mathrm{ml}, 4.8 \mathrm{mmol}, 4.8 \mathrm{eq})$ was added dropwise to a solution of 15 (293 $\mathrm{mg}, 1 \mathrm{mmol}$, $1.0 \mathrm{eq})$ in toluene $(15 \mathrm{ml})$. After stirring for $15 \mathrm{~min}$ at room temperature, a dark yellow precipitate was formed which was filtered and washed with toluene $(3 \times 5 \mathrm{ml})$. The pure product $(342 \mathrm{mg}$, $934 \mu \mathrm{mol}, 93.4 \%$ ) was dried under reduced pressure to obtain a bright yellow powder.

Suitable crystals for X-ray analysis were formed after a recrystallisation from methanol.

Reverse reaction to 15: The deprotonation of $16(235 \mathrm{mg}, 641 \mu \mathrm{mol}, 1.0 \mathrm{eq})$ was carried out in a saturated aqueous $\mathrm{NaHCO}_{3}$ solution $(10 \mathrm{ml}, 11.4 \mathrm{mmol}, 17.8 \mathrm{eq})$ which was stirred for $30 \mathrm{~min}$. The product was extracted with ethyl acetate $(20 \mathrm{ml})$ and the organic phase was washed with aqueous $\mathrm{NaHCO}_{3}$ solution (2x $10 \mathrm{ml}$ ). The organic phase was dried over $\mathrm{MgSO}_{4}$, filtered and the volatile compounds were removed under reduced pressure. 15 (155 mg, $528 \mu \mathrm{mol}, 82.4 \%$ ) could be reobtained as a yellow-brown solid.

Chemical formula: $\quad \mathrm{C}_{19} \mathrm{H}_{25} \mathrm{~N}_{3} \mathrm{Cl}_{2}$

Molecular weight: $\quad 366.33 \mathrm{~g} / \mathrm{mol}$<smiles>CN(C)Cc1cccc2cc3cccc(CN(C)C)c3nc12</smiles>

\section{${ }^{1}$ H NMR}

$\left(300 \mathrm{MHz}, \mathrm{DMSO}-d_{6}\right): \quad \delta[\mathrm{ppm}]=10.42\left(\mathrm{~s}, 2 \mathrm{H}, \mathrm{NH}_{2}\right), 9.36\left(\mathrm{~s}, 1 \mathrm{H}, \mathrm{H}_{9}\right), 8.38\left(\mathrm{~d},{ }^{3} J=8.2 \mathrm{~Hz}\right.$, $\left.2 \mathrm{H}, \mathrm{H}_{1,8}\right), 8.17\left(\mathrm{~d},{ }^{3} J=6.4 \mathrm{~Hz}, 2 \mathrm{H}, \mathrm{H}_{3,6}\right), 7.77\left(\mathrm{dd},{ }^{3} J=8.2,7.0 \mathrm{~Hz}\right.$, $\left.2 \mathrm{H}, \mathrm{H}_{2,7}\right), 5.16\left(\mathrm{~d},{ }^{3} \mathrm{~J}=5.7 \mathrm{~Hz}, 2 \mathrm{H}, \mathrm{CH}_{2}\right), 2.91\left(\mathrm{~d},{ }^{3} \mathrm{~J}=4.8 \mathrm{~Hz} 12 \mathrm{H}\right.$, $\mathrm{CH}_{3}$. 


\section{${ }^{13}$ C NMR}

$\left(75 \mathrm{MHz}, \mathrm{DMSO}-d_{6}\right): \quad \delta[\mathrm{ppm}]=146.06\left(2 \mathrm{C}, \mathrm{C}_{4 \mathrm{a}, 10 \mathrm{a}}\right), 138.56\left(1 \mathrm{C}, \mathrm{C}_{9}\right), 135.77\left(2 \mathrm{C}, \mathrm{C}_{3,6}\right)$, $131.17\left(2 \mathrm{C}, \mathrm{C}_{1,8}\right), 128.07\left(2 \mathrm{C}, \mathrm{C}_{4,5}\right), 126.45\left(2 \mathrm{C}, \mathrm{C}_{8 \mathrm{a}, 9 \mathrm{a}}\right), 125.70(2 \mathrm{C}$, $\left.\mathrm{C}_{2,7}\right), 55.90\left(2 \mathrm{C}, \mathrm{CH}_{2}\right), 42.54\left(4 \mathrm{C}, \mathrm{CH}_{3}\right)$.

\section{ESI MS}

$\mathrm{m} / \mathrm{z}(\%)$ :

$400(27)[\mathrm{M}+\mathrm{Cl}]^{-}, 364(18)[\mathrm{M}-\mathrm{H}]^{-}$.

\section{Elemental analysis}

in \% (calculated): $\quad$ C: 61.46 (62.29), H: 6.61 (6.88), N: 11.18 (11.47), Cl: 20.35 (19.36).

\subsubsection{Synthesis of 4,5-bis(dimethylaminemethylene)acridine dibromido zinc(II) (17)}

15 (175 mg, $596 \mu \mathrm{mol}, 1.0 \mathrm{eq})$ was dissolved in THF (1 ml) and a solution of $\mathrm{ZnBr}_{2}$ (134 mg, $596 \mu \mathrm{mol}, 1.0 \mathrm{eq})$ in THF $(0.2 \mathrm{ml})$ was added dropwise. Thereby, the zinc complex precipitated immediately. The suspension was filtrated and washed several times with THF. The pure product (145 mg, $280 \mu \mathrm{mol}, 47.0 \%$ ) could be obtained as a yellow powder.

17 was dissolved in a THF/DMF (9.3:1) mixture and heated up to the boiling point of THF. After two weeks at room temperature, suitable crystals for X-ray analysis could be obtained.

Reverse reaction to 15: 17 (139 mg, $268 \mu \mathrm{mol}, 1.0 \mathrm{eq})$ was suspended in an aq. $\mathrm{NaHCO}_{3}$ solution $(5 \%, 10 \mathrm{ml})$ when $\mathrm{Na}_{2} \mathrm{H}_{2}$ EDTA $(360 \mathrm{mg}, 1.07 \mathrm{mmol}, 4.0 \mathrm{eq})$, dissolved in an aq. $\mathrm{NaHCO}_{3}$ solution $(10 \%, 6 \mathrm{ml})$, was added. The suspension was stirred at room temperature for $45 \mathrm{~min}$. The product was extracted with ethyl acetate $(2 \mathrm{x} 4 \mathrm{ml})$ and the gathered organic phases were washed with an aq. saturated $\mathrm{NaHCO}_{3}$ solution ( $3 \times 10 \mathrm{ml}$ ). The organic phases were dried over $\mathrm{MgSO}_{4}$ and filtered. After the solvent was removed under reduced pressure, 15 (74 mg, $252 \mu \mathrm{mol}, 94 \%$ ) was reobtained as a yellow-brown solid.

\section{Chemical formula: $\quad \mathrm{C}_{19} \mathrm{H}_{23} \mathrm{~N}_{3} \mathrm{ZnBr}_{2}$ \\ Molecular weight: $\quad 518.61 \mathrm{~g} / \mathrm{mol}$}

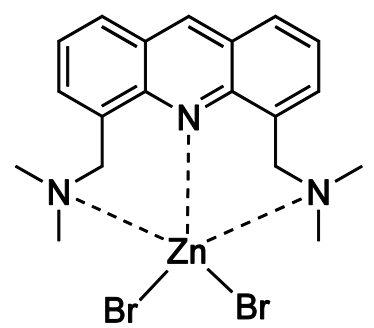

\section{${ }^{1}$ H NMR}

$\left(400 \mathrm{MHz}, \mathrm{DMF}-d_{7}, \quad \delta[\mathrm{ppm}]=9.25\left(\mathrm{~s}, 1 \mathrm{H}, \mathrm{H}_{9}\right), 8.22\left(\mathrm{~d},{ }^{3} J=8.3 \mathrm{~Hz}, 2 \mathrm{H}, \mathrm{H}_{1,8}\right), 8.01(\mathrm{~d}\right.$, $\left.80^{\circ} \mathrm{C}\right)$ : $\left.{ }^{3} J=7.1 \mathrm{~Hz}, 2 \mathrm{H}, \mathrm{H}_{3,6}\right), 7.69\left(\mathrm{dd},{ }^{3} J=8.3,7.1 \mathrm{~Hz}, 2 \mathrm{H}, \mathrm{H}_{2,7}\right), 4.52(4 \mathrm{H}$, $\mathrm{CH}_{2}$ ), 2.62 (s, $12 \mathrm{H}, \mathrm{CH}_{3}$ ).

$\left(400 \mathrm{MHz}, \mathrm{DMF}-d_{7}, \quad \delta[\mathrm{ppm}]=9.57\left(\mathrm{~s}, 1 \mathrm{H}, \mathrm{H}_{9}\right), 8.39\left(\mathrm{dd},{ }^{3} \mathrm{~J}=8.0 \mathrm{~Hz},{ }^{4} \mathrm{~J}=1.6 \mathrm{~Hz}, 2 \mathrm{H}\right.\right.$, $\left.-30{ }^{\circ} \mathrm{C}\right)$ : $\left.\mathrm{H}_{1,8}\right), 8.11\left(\mathrm{dd},{ }^{3} J=6.1 \mathrm{~Hz},{ }^{4} J=1.3 \mathrm{~Hz}, 2 \mathrm{H}, \mathrm{H}_{3,6}\right), 7.76\left(\mathrm{dd},{ }^{3} J=8.4\right.$, $\left.6.8 \mathrm{~Hz}, 2 \mathrm{H}, \mathrm{H}_{2,7}\right), 5.46\left(\mathrm{~d},{ }^{2} J=11.9 \mathrm{~Hz}, 2 \mathrm{H}, \mathrm{CH}_{2}\right), 3.86(\mathrm{~d}$, 
$\left.{ }^{2} J=12.1 \mathrm{~Hz}, 2 \mathrm{H}, \mathrm{CH}_{2}\right), 3.19\left(\mathrm{~s}, 6 \mathrm{H}, \mathrm{CH}_{3}\right), 2.38\left(\mathrm{~s}, 6 \mathrm{H}, \mathrm{CH}_{3}\right)$.

${ }^{13}$ C NMR

$\left(100 \mathrm{MHz}, \mathrm{DMF}-d_{7}, \quad \delta[\mathrm{ppm}]=148.65\left(2 \mathrm{C}, \mathrm{C}_{4 \mathrm{a}, 10 \mathrm{a}}\right), 141.97\left(1 \mathrm{C}, \mathrm{C}_{9}\right), 135.45\left(2 \mathrm{C}, \mathrm{C}_{3,6}\right)\right.$, $\left.-30{ }^{\circ} \mathrm{C}\right)$ :

$130.52\left(2 \mathrm{C}, \mathrm{C}_{1,8}\right), 127.68\left(2 \mathrm{C}, \mathrm{C}_{8 \mathrm{a}, 9 \mathrm{a}}\right), 126.44\left(2 \mathrm{C}, \mathrm{C}_{2,7}\right), 63.13(2 \mathrm{C}$, $\left.\mathrm{CH}_{2}\right), 48.91\left(2 \mathrm{C}, \mathrm{CH}_{3}\right), 46.37\left(2 \mathrm{C}, \mathrm{CH}_{3}\right)$.

${ }^{15}$ N NMR

(40.6 MHz, DMF- $d_{7}, \quad \delta[\mathrm{ppm}]=-346.7$ (taken from $\left.{ }^{1} \mathrm{H},{ }^{15} \mathrm{~N}-\mathrm{HMBC}\right)$. $\left.-30{ }^{\circ} \mathrm{C}\right)$ :

Elemental analysis

in $\%$ (calculated): C: 44.00 (44.00), H: 4.50 (4.47), N: 8.10 (8.10), Br: 30.00 (30.81).

\section{TOF ESI MS}

$\mathrm{m} / \mathrm{z}(\%)$ :

$$
\begin{aligned}
& 957.01(0.6)\left[\left(\mathrm{C}_{19} \mathrm{H}_{23} \mathrm{~N}_{3}\right)_{2} \mathrm{Zn}_{2} \mathrm{Br}_{3}\right]^{+}, 813.15(1.8)\left[\left(\mathrm{C}_{19} \mathrm{H}_{23} \mathrm{~N}_{3}\right)_{2} \mathrm{ZnBr}_{2} \mathrm{H}\right]^{+}, \\
& 438.05(100)\left[\mathrm{C}_{19} \mathrm{H}_{23} \mathrm{~N}_{3} \mathrm{ZnBr}\right]^{+}, 300.22(34)\left[\mathrm{C}_{19} \mathrm{H}_{23} \mathrm{~N}_{3} \mathrm{Li}\right]^{+}, 294.20(93) \\
& {\left[\mathrm{C}_{19} \mathrm{H}_{24} \mathrm{~N}_{3}\right]^{+} .}
\end{aligned}
$$

\subsubsection{Synthesis of 4,5-bis(dimethylaminemethylene)acridine dibromido cadmium(II) (19)}

15 (141 mg, $480 \mu \mathrm{mol}, 1.0 \mathrm{eq})$ was dissolved in methanol $(0.5 \mathrm{ml})$ and a solution of $\mathrm{CdBr}_{2}$ (131 mg, $480 \mu \mathrm{mol}, 1.0 \mathrm{eq})$ in methanol $(1.5 \mathrm{ml})$ was poured into. During the addition, the cadmium complex precipitated immediately. Another $5 \mathrm{ml}$ of methanol was added and the suspension was filtrated. The residue was washed several times with methanol $(3 \mathrm{x} 5 \mathrm{ml})$ and dried under reduced pressure. The pure product ( $245 \mathrm{mg}, 433 \mu \mathrm{mol}, 90.2 \%$ ) was obtained as a yellow powder.

Suitable crystals for X-ray diffraction were obtained through crystallisation from a THF/DMF (1.5:1) mixture by slowly cooling the boiling solution to room temperature.

Chemical formula: $\quad \mathrm{C}_{19} \mathrm{H}_{23} \mathrm{~N}_{3} \mathrm{CdBr}_{2}$

Molecular weight: $\quad 565.63 \mathrm{~g} / \mathrm{mol}$

${ }^{1}$ H NMR<smiles></smiles>

$\left(400 \mathrm{MHz}, \mathrm{DMF}-d_{7}, \quad \delta[\mathrm{ppm}]=9.32\left(\mathrm{~s}, 1 \mathrm{H}, \mathrm{H}_{9}\right), 8.28\left(\mathrm{~d},{ }^{3} J=8.5 \mathrm{~Hz}, 2 \mathrm{H}, \mathrm{H}_{1,8}\right), 8.08-7.99\right.$ $\left.80{ }^{\circ} \mathrm{C}\right): \quad\left(\mathrm{m}, 2 \mathrm{H}, \mathrm{H}_{3,6}\right), 7.71\left(\mathrm{dd},{ }^{3} \mathrm{~J}=8.5,6.8 \mathrm{~Hz}, 2 \mathrm{H}, \mathrm{H}_{2,7}\right), 4.56\left(\mathrm{~s}, 4 \mathrm{H}, \mathrm{CH}_{2}\right)$, $2.74\left(\mathrm{~s}, 12 \mathrm{H}, \mathrm{CH}_{3}\right)$. 


\section{${ }^{113}$ Cd NMR}

(66.6 MHz, DMF- $d_{7}, \quad \delta[\mathrm{ppm}]=-345.5$ (taken from $\left.{ }^{1} \mathrm{H},{ }^{113} \mathrm{Cd}-\mathrm{HMBC}\right)$. $\left.-30{ }^{\circ} \mathrm{C}\right)$ :

\section{TOF ESI MS}

$\mathrm{m} / \mathrm{z}(\%)$ :

$$
\begin{aligned}
& 1050.96 \quad(0.4) \quad\left[\left(\mathrm{C}_{19} \mathrm{H}_{23} \mathrm{~N}_{3}\right)_{2} \mathrm{Cd}_{2} \mathrm{Br}_{3}\right]^{+}, \quad 861.15 \\
& {\left[\left(\mathrm{C}_{19} \mathrm{H}_{23} \mathrm{~N}_{3}\right)_{2} \mathrm{CdBr}_{2} \mathrm{H}\right]^{+}, 587.40 \text { (9.2) }\left[\left(\mathrm{C}_{19} \mathrm{H}_{23} \mathrm{~N}_{3}\right)_{2} \mathrm{H}\right]^{+}, 542.34 \text { (10.8) }} \\
& {\left[\mathrm{C}_{36} \mathrm{H}_{40} \mathrm{~N}_{5}\right]^{+}, \quad 486.02 \quad(15) \quad\left[\left(\mathrm{C}_{19} \mathrm{H}_{23} \mathrm{~N}_{3}\right) \mathrm{CdBr}\right]^{+}, \quad 294.21} \\
& {\left[\left(\mathrm{C}_{19} \mathrm{H}_{23} \mathrm{~N}_{3}\right) \mathrm{H}\right]^{+} \text {. }}
\end{aligned}
$$

\section{Elemental analysis}

in \% (calculated):

C: 40.28 (40.35), H: 4.31 (4.10), N: 7.20 (7.43), Cd: 19.00 (19.87).

\subsubsection{Synthesis of 4,5-bis(dimethylaminemethylene)acridine tetrakis( $\mu$-acetate) bis(acetate) zinc(II) (20)}

Anhydrous zinc acetate $(100 \mathrm{mg}, 545 \mu \mathrm{mol}, 1.6 \mathrm{eq})$ was dissolved in dem. water $(0.5 \mathrm{ml})$ and added to a solution of $\mathbf{1 5}(100 \mathrm{mg}, 341 \mu \mathrm{mol}, 1.0 \mathrm{eq})$ in methanol. The volatile compounds of the resulting suspension were removed in vacuo and a mixture of toluene with DMF was used to dissolve the product at room temperature. Suitable crystals for X-ray analysis were formed at $-30{ }^{\circ} \mathrm{C}$ after ten days.

Reverse reaction to 15: The free ligand was reobtained by the application of the same procedure to $\mathbf{2 0}$ as for 17. A direct yield cannot be reported since $\mathbf{2 0}$ was not completely isolated before. Nevertheless, $78 \%$ (78 mg, $266 \mu \mathrm{mol}$ ) of the used ligand 15 was gained back.

Chemical formula: $\quad \mathrm{C}_{31} \mathrm{H}_{41} \mathrm{~N}_{3} \mathrm{O}_{12} \mathrm{Zn}_{3}$

Molecular weight: $\quad 843.82 \mathrm{~g} / \mathrm{mol}$

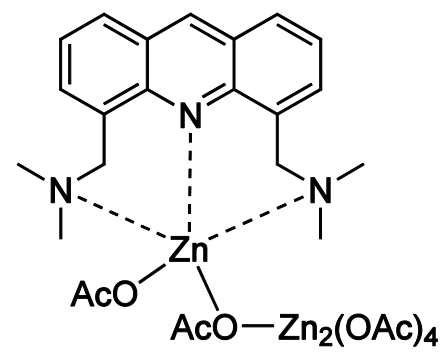





\section{CRYSTALLOGRAPHIC SECTION}

\subsection{Crystal selection and manipulation}

Suitable single crystals for X-ray structural analysis were selected from a Schlenk flask under an argon atmosphere and covered with perfluorated polyether oil on a microscope slide, which was cooled with a nitrogen gas flow using the X-TEMP2 device. ${ }^{[159]}$ An appropriate crystal was selected using a polarising microscope, mounted on the tip of a MiTeGen MicroMount, fixed to a goniometer head and shock-cooled by the crystal cooling device.

\subsection{Data collection and processing}

The data for the determined structures were collected on four different diffractometers. The datasets using $\mathrm{Cu}-\mathrm{K} \alpha$ radiation $(\lambda=1.54178 \AA$ ) were recorded on a Bruker MacScience rotating anode diffractometer equipped with a SMART6000 CCD detector. In the case of Mo- $K_{\alpha}$ radiation $(\lambda=0.71073 \AA)$, either an Incoatec Mo microsource ${ }^{[160]}$ with mirror optics or a rotating anode with turbo X-ray source was used. The smallest wavelength with $\lambda=0.56086 \AA$ is generated by an Incoatec Ag microfocus source with Incoatec Quazar mirror optics. The Mo and Ag diffractometers were equipped with an APEX II detector mounted on a three circle D8 goniometer. A low-temperature device was used in every case keeping the crystal temperature at $100 \mathrm{~K}$ during the experiment. The data were integrated with $S A I N T^{161]}$ and an empirical absorption correction was applied $(S A D A B S)^{[83]} . X P R E P^{[162]}$ was used to for the examination of data statistics and space group determination.

\subsection{Structure solution and refinement}

The structures were solved by direct methods using $S H E L X T^{[163]}$ and refined by full-matrix leastsquares methods against $F^{2}(S H E L X L)^{[164]}$ within the ShelXle GUI. ${ }^{[165]}$ All non-hydrogen atoms were refined with anisotropic displacement parameters. The C-bonded hydrogen atoms were refined isotropically on calculated positions using a riding model with their $U_{\text {iso }}$ values constrained to equal 1.5 times the $U_{\text {eq }}$ of their pivot atoms for terminal $\mathrm{sp}^{3}$ carbon atoms and 1.2 times for all other carbon atoms. The positions of hydrogen atoms attached to heteroatoms were found in the Fourier-density-difference map. They were refined either with the DFIX command to set the distance to tabulated values or if similar bonding situations were present $(\mathrm{N}-\mathrm{H}, \mathrm{O}-\mathrm{H})$, the restraint SADI was used to align the distances. ${ }^{[84]}$

In the absence of restraints, the only data the structural model is refined against are the measured intensities in the form of squared structure factors. Structure factors are calculated from the atomic model and the so-calculated intensities are then compared with the measured intensities, and the best model is the one that minimises $M\left(p_{i}, k\right)$ (Eq. 5.1) using the weights $w$ defined in Eq. 5.2 ( $p_{i}$ : structural parameter; $k$ : scale factor).

Eq. 5.1

$$
M\left(p_{i}, k\right)=\sum w\left[k\left|F_{\text {obs }}\right|^{2}-\left|F_{\text {calc }}\right|^{2}\right]^{2}=\min
$$

Eq. 5.2

$$
w^{-1}=\sigma^{2}\left(F_{\mathrm{obs}}^{2}\right)+(g 1 \times P)^{2}+g 2 \times P \quad \text { with } P=\left(\frac{\operatorname{Max}\left(F_{\mathrm{obs}}^{2}, 0\right)+2 F_{\mathrm{calc}}^{2}}{3}\right)
$$


The results of the refinements were verified by comparison of the calculated and the observed structure factors. Commonly used criteria are the residuals $R_{1}$ (Eq. 5.3) and $w R_{2}$ (Eq. 5.4). The $w R_{2}$ is more significant because the model is refined against $F^{2}$.

Eq. 5.3

$$
R_{1}=\frac{\sum|| F_{\text {obs }}|-| F_{\text {calc }}||}{\sum\left|F_{\text {obs }}\right|}
$$

$$
w R_{2}=\sqrt{\frac{\sum w\left(F_{\mathrm{obs}}^{2}-F_{\mathrm{calc}}^{2}\right)^{2}}{\sum w\left(F_{\mathrm{obs}}^{2}\right)^{2}}}
$$

Additionally, the goodness-of-fit ( $\mathrm{GoF})$ a figure or merit showing the relation between deviation of $F_{\text {calc }}$ from $F_{\text {obs }}$ and the over-determination of refined parameters is calculated (Eq. $5.5 ; N_{\mathrm{R}}$ : number of reflections; $N_{\mathrm{P}}$ : number of parameters).

Eq. 5.5

$$
\mathrm{GoF}=\sqrt{\frac{\sum w\left(F_{\mathrm{obs}}^{2}-F_{\text {calc }}^{2}\right)^{2}}{N_{\mathrm{R}}-N_{\mathrm{P}}}}
$$

The residual densities from difference Fourier analysis should be low. Due to the model restrictions, the residuals are normally found in the bonding regions. Higher residuals for heavy scatterers are acceptable as they arise mainly from absorption effects and Fourier truncation errors due to the limited recorded resolution range. The highest peak and deepest hole from difference Fourier analysis are listed in the crystallographic tables.

Additionally, the orientation, size and ellipticity of the ADPs show the quality of the model. Ideally, the ADPs should be oriented perpendicular to the bonds, be equal in size and show little ellipticity. ${ }^{[166]}$ All graphics were generated and plotted with the $X P^{[167]}$ program at the $50 \%$ probability level.

\subsection{Treatment of disorder}

Structures containing disordered fragments were refined using constraints and restraints. Constraints used within this work are, for example, the site occupation factor and the AFIX instruction, which defines and constrains rigid groups. Mathematically, restraints are treated as additional experimental observations, thus increasing the number of data to refine against. In the presence of restraints, the minimisation function changes as follows:

Eq. 5.6

$$
M=\sum w\left(F_{\text {obs }}^{2}-F_{\text {calc }}^{2}\right)^{2}+\sum w_{r}\left(R_{\text {target }}-R_{\text {calc }}\right)^{2},
$$

whereby $R_{\text {calc }}$ is the actual value of the restrained quantity.

The geometries of chemically equivalent but crystallographically independent fragments can be fitted to each other by distance restraints. Especially, the 1,2-distances (bond lengths) and 1,3distances (bond angles) are set to be equal within their effective standard deviations. This is helpful for refining disordered positions as the averaging of equivalent fragments implements chemical information and stabilises the refinement. Within this work, disordered moieties were refined using distance restraints (SADI and SAME) and anisotropic displacement parameter restraints (SIMU and RIGU). ${ }^{[84]}$ 


\subsection{Determined structures}

\subsubsection{4,5-Bis(bromomethylene)-9-methylacridine (9)}

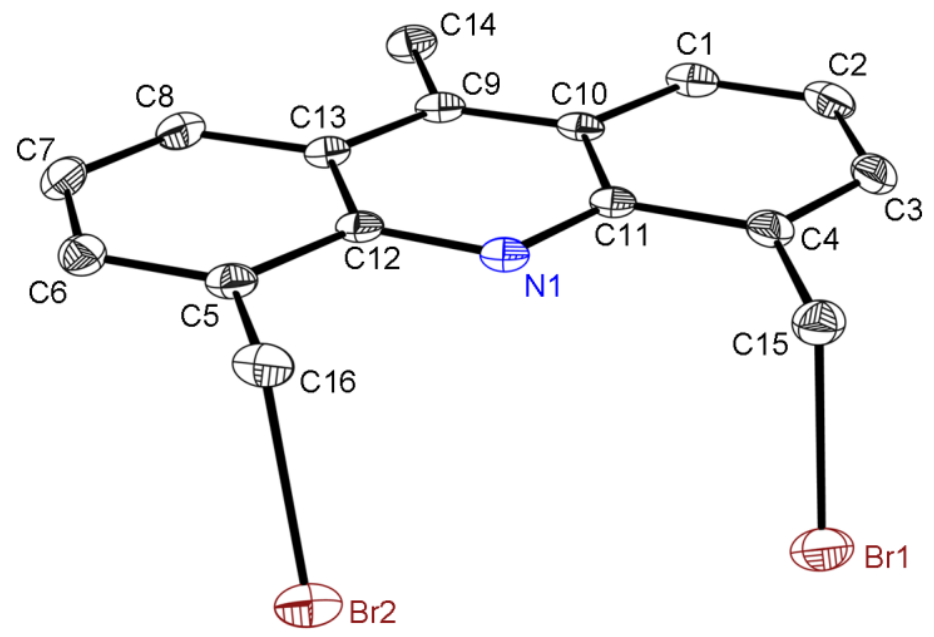

Figure 5.1: Solid state structure of 9 . The anisotropic displacement parameters are depicted at the 50\% probability level. All hydrogen atoms are omitted for clarity.

Table 5.1: Crystallographic information of 9.

\begin{tabular}{ll|ll}
\hline CCDC No. & $\mathrm{n} / \mathrm{a}$ & Density (calculated) & $1.836 \mathrm{Mg} / \mathrm{m}^{3}$ \\
Structure code & $9-\mathrm{MeAcrCH}_{2} \mathrm{Br}$ & Absorption coefficient & $5.896 \mathrm{~mm}^{-1}$ \\
Empirical formula & $\mathrm{C}_{16} \mathrm{H}_{13} \mathrm{Br}_{2} \mathrm{~N}$ & $F(000)$ & 744 \\
Formula weight & 379.09 & Crystal size & $0.185 \mathrm{x} 0.150 \times 0.122 \mathrm{~mm}^{3}$ \\
Temperature & $100(2) \mathrm{K}$ & $\Theta$ range for data collection & 2.313 to $27.875^{\circ}$ \\
Wavelength & $0.71073 \AA$ & Reflections collected & 37295 \\
Crystal system & monoclinic & Independent reflections & $3268\left[R_{\mathrm{int}}=0.0238\right]$ \\
Space group & $P 2_{1} / n$ & Completeness to $\Theta_{\text {max }}$ & $99.9 \%$ \\
Unit cell dimensions & $a=9.598(2) \AA$ & Max. and min. transmission & 0.4308 and 0.3717 \\
& $b=8.378(2) \AA$ & Data / restraints / parameters & $3268 / 0 / 173$ \\
& $c=17.193(3) \AA$ & Goodness-of-fit on $F^{2}$ & 1.060 \\
& $\alpha=90^{\circ}$ & Final $R$ indices $[I>2 \sigma(I)]$ & $R_{1}=0.0198, \mathrm{w} R_{2}=0.0529$ \\
& $\beta=97.32(2)^{\circ}$ & $R$ indices (all data) & $R_{1}=0.0216, \mathrm{w} R_{2}=0.0536$ \\
& $\gamma=90^{\circ}$ & Absolute structure parameter & $\mathrm{n} / \mathrm{a}$ \\
Volume & $1.3713(5) \mathrm{nm}{ }^{3}$ & Extinction coefficient & $\mathrm{n} / \mathrm{a}$ \\
\hline & 4 & Largest diff. peak and hole & 0.923 and $-0.698 \mathrm{e}^{-3}$ \\
\hline
\end{tabular}




\subsubsection{4,5-Bis(di(2'-methylthiobenzyl)aminemethylene)-9-methylacridine (10)}

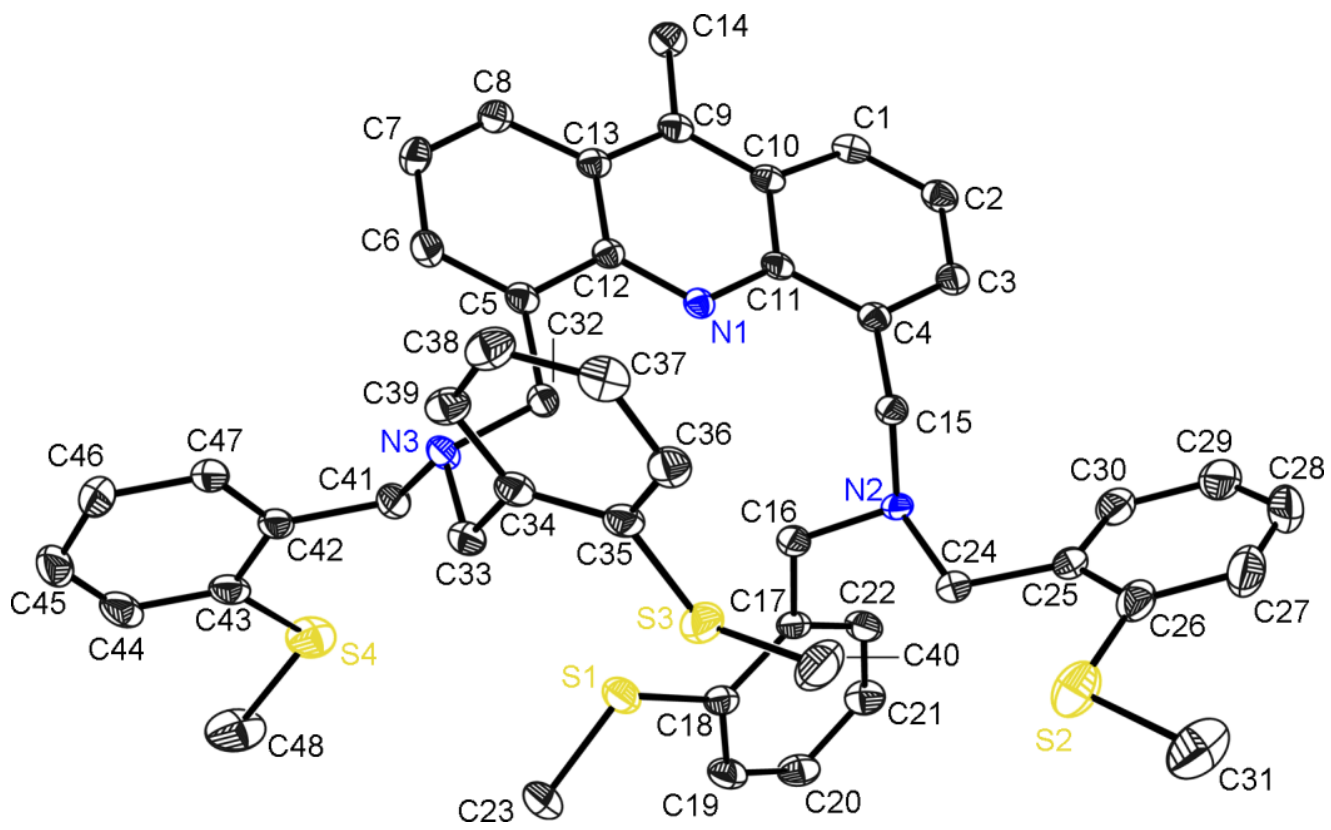

Figure 5.2: Solid state structure of 10. The anisotropic displacement parameters are depicted at the 50\% probability level. All hydrogen atoms are omitted for clarity. In proximity to C2 and C7 unusual residual electron density was noticed. This phenomenon could not be explained reasonable but was also observed at a solid state structure of an acridine unit treated with bromine. Maybe C2 and C7 are brominated by approx. $1 \%$ due to the forming of $\mathrm{HBr}$ during the reaction process to $\mathbf{1 0}$. Furthermore, the electron density at two of the four sulfur atoms (S1 and S3) is described too high.

Table 5.2: Crystallographic information of $\mathbf{1 0}$.

\begin{tabular}{ll|ll}
\hline CCDC No. & $\mathrm{n} / \mathrm{a}$ & Density (calculated) & $1.294 \mathrm{Mg} / \mathrm{m}^{3}$ \\
Structure code & AcrNBnSMe & Absorption coefficient & $0.271 \mathrm{~mm}^{-1}$ \\
Empirical formula & $\mathrm{C}_{48} \mathrm{H}_{49} \mathrm{~N}_{3} \mathrm{~S}_{4}$ & $F(000)$ & 844 \\
Formula weight & 796.14 & Crystal size & $0.460 \mathrm{x} 0.220 \times 0.060 \mathrm{~mm}^{3}$ \\
Temperature & $100(2) \mathrm{K}$ & $\Theta$ range for data collection & 1.210 to $26.037^{\circ}$ \\
Wavelength & $0.71073 \AA$ & Reflections collected & 38266 \\
Crystal system & triclinic & Independent reflections & $7945\left[R_{\text {int }}=0.0255\right]$ \\
Space group & $P \overline{1}$ & Completeness to $\Theta_{\text {max }}$ & $98.5 \%$ \\
Unit cell dimensions & $a=10.709(2) \AA$ & Max. and min. transmission & $0.7453 \mathrm{and} 0.7081$ \\
& $b=11.638(2) \AA$ & Data / restraints / parameters & $7945 / 24 / 501$ \\
& $c=17.300(3) \AA$ & Goodness-of-fit on $F^{2}$ & 1.026 \\
& $\alpha=93.28(2)^{\circ}$ & Final $R$ indices $[I>2 \sigma(I)]$ & $R_{1}=0.0383, \mathrm{w} R_{2}=0.0912$ \\
& $\beta=101.85(2)^{\circ}$ & $R$ indices (all data) & $R_{1}=0.0499, \mathrm{w} R_{2}=0.0989$ \\
& $\gamma=103.02(2)^{\circ}$ & Absolute structure parameter & $\mathrm{n} / \mathrm{a}$ \\
Volume & $2.0438(7) \mathrm{nm}^{3}$ & Extinction coefficient & $\mathrm{n} / \mathrm{a}$ \\
$Z$ & 2 & Largest diff. peak and hole & 1.080 and $-0.388 \mathrm{e} \AA^{-3}$ \\
\hline
\end{tabular}




\subsubsection{4,5-Bis $(N$-methyl- $N$-phenylaminemethylene)acridine hydrochloride (14)}

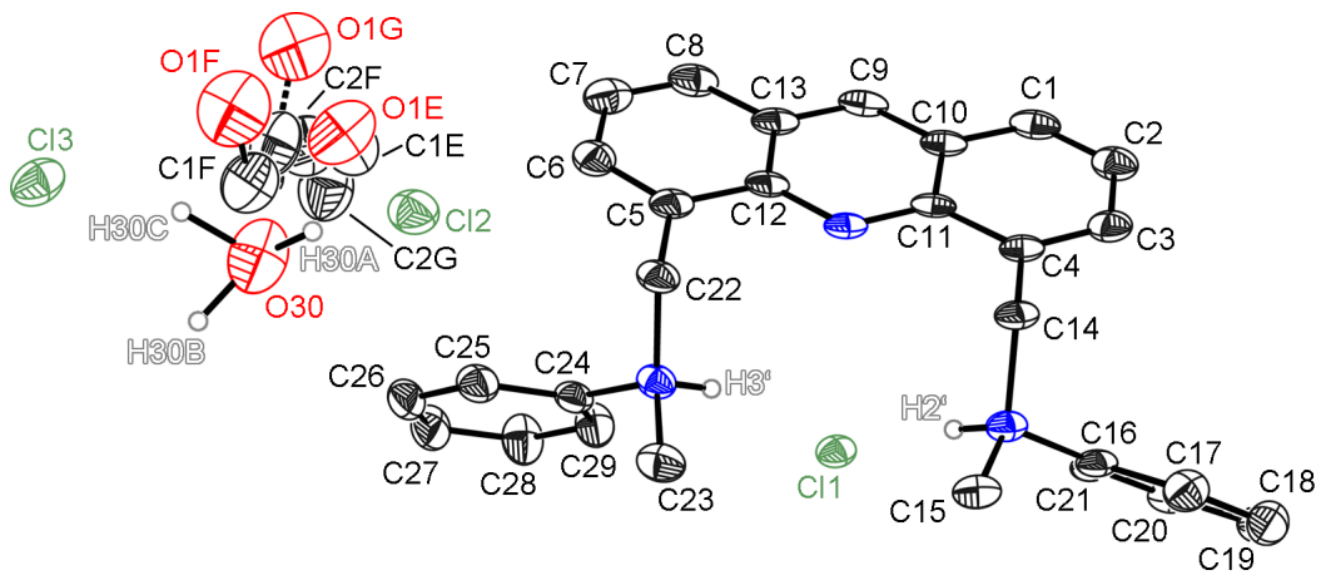

Figure 5.3: Solid state structure of 14. The anisotropic displacement parameters are depicted at the 50\% probability level. Not freely refined hydrogen atoms are omitted for clarity. The chlorine atom $\mathrm{Cl} 3$ lies on a special position with a site occupation factor (sof) of 0.5 and the ethanol solvent molecule is disordered over three positions (sof: $0.762(3), 0.131(3), 0.107(3)$ ). Therefore, the hydrogen atoms of the oxygen atoms (O1) could not be refined. The position of the hydrogen atoms at $\mathrm{O} 30$ were refined freely but more residual electron density could be found in its environment. Probably, the $\mathrm{H}_{3} \mathrm{O}^{+}$molecule is disordered, too, or one hydrogen atom is connected to the $\mathrm{Cl} 2$ atom, resulting in the neutral compounds $\mathrm{H}_{2} \mathrm{O}$ and $\mathrm{HCl}$. This issue could not be solved with the available data.

Table 5.3: Crystallographic information of 14.

\begin{tabular}{ll|ll}
\hline CCDC No. & $\mathrm{n} / \mathrm{a}$ & Density (calculated) & $1.270 \mathrm{Mg} / \mathrm{m}^{3}$ \\
Structure code & AcrNMePh_HCl & Absorption coefficient & $0.157 \mathrm{~mm}^{-1}$ \\
Empirical formula & $\mathrm{C}_{62} \mathrm{H}_{76} \mathrm{Cl}_{5} \mathrm{~N}_{6} \mathrm{O}_{4}$ & $F(000)$ & 2420 \\
Formula weight & 1146.54 & Crystal size & $0.196 \mathrm{x} 0.087 \mathrm{x} 0.044 \mathrm{~mm}^{3}$ \\
Temperature & $100(2) \mathrm{K}$ & $\Theta$ range for data collection & 1.927 to $18.233^{\circ}$ \\
Wavelength & $0.56086 \AA$ & Reflections collected & 91533 \\
Crystal system & monoclinic & Independent reflections & $4343\left[R_{\text {int }}=0.0723\right]$ \\
Space group & $C 2 / c$ & Completeness to $\Theta_{\text {max }}$ & $99.7 \%$ \\
Unit cell dimensions & $a=24.851(3) \AA$ & Max. and min. transmission & 0.7442 and 0.6867 \\
& $b=11.214(2) \AA$ & Data / restraints / parameters & $4343 / 221 / 426$ \\
& $c=24.692(3) \AA$ & Goodness-of-fit on $F^{2}$ & 1.030 \\
& $\alpha=90^{\circ}$ & Final $R$ indices $[I>2 \sigma(I)]$ & $R_{1}=0.0416, \mathrm{w} R_{2}=0.0954$ \\
& $\beta=119.56(2)^{\circ}$ & $R$ indices (all data) & $R_{1}=0.0531, \mathrm{w} R_{2}=0.1029$ \\
& $\gamma=90^{\circ}$ & Absolute structure parameter & $\mathrm{n} / \mathrm{a}$ \\
Volume & $5.9855(18) \mathrm{nm}^{3}$ & Extinction coefficient & $\mathrm{n} / \mathrm{a}$ \\
\hline
\end{tabular}




\subsection{4 $\left[\left(\mathrm{Me}_{2} \mathrm{NHCH}_{2}\right)_{2} \mathrm{Acr}\right] \mathrm{Cl}_{2} \cdot(\mathrm{MeOH})(16)$}

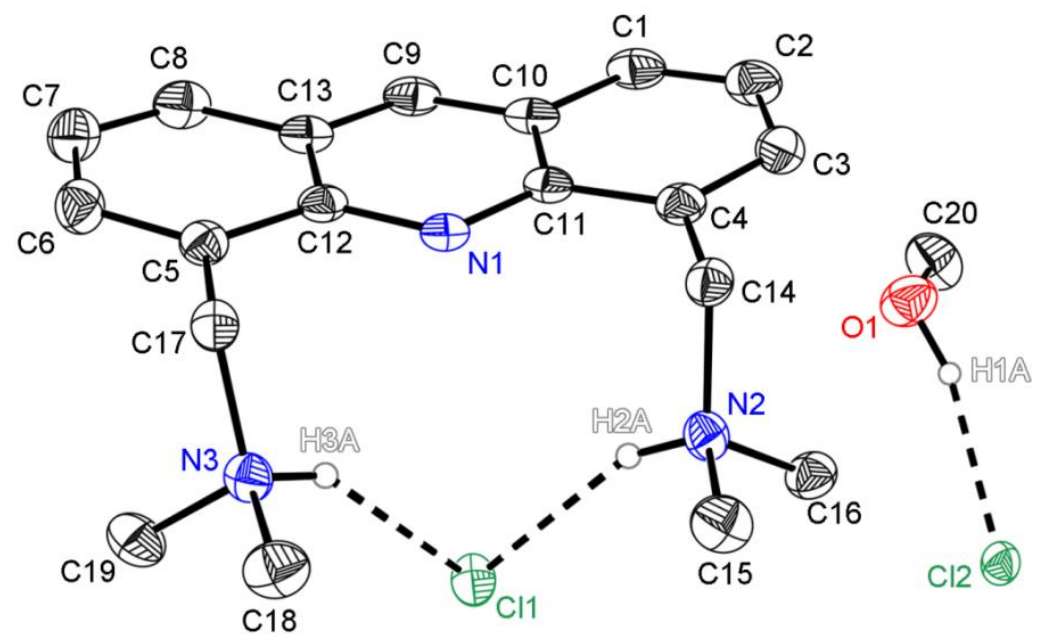

Figure 5.4: Solid state structure of 16. The anisotropic displacement parameters are depicted at the 50\% probability level. The positions of the hydrogen atoms H1A, H2A and H3A were refined freely whereas the remaining hydrogen atoms are omitted for clarity. The structure is published in Dalton Trans. 2016, 45, 5689-5699. ${ }^{[111]}$

Table 5.4: Crystallographic information of $\mathbf{1 6 .}$

\begin{tabular}{ll|ll}
\hline CCDC No. & 1423219 & Density (calculated) & $1.271 \mathrm{Mg} / \mathrm{m}^{3}$ \\
Structure code & AcrNMe $2 \mathrm{HCl}$ & Absorption coefficient & $2.905 \mathrm{~mm}^{-1}$ \\
Empirical formula & $\mathrm{C}_{20} \mathrm{H}_{29} \mathrm{Cl}_{2} \mathrm{~N}_{3} \mathrm{O}$ & $F(000)$ & 848 \\
Formula weight & $398.36 \mathrm{~g} / \mathrm{mol}$ & Crystal size & $0.10 \mathrm{x} 0.10 \times 0.05 \mathrm{~mm}^{3}$ \\
Temperature & $100(2) \mathrm{K}$ & $\Theta$ range for data collection & 28.413 to $66.420^{\circ}$ \\
Wavelength & $1.54178 \AA$ & Reflections collected & 3602 \\
Crystal system & orthorhombic & Independent reflections & $1723\left[R_{\text {int }}=0.0163\right]$ \\
Space group & $P n a 2_{1}$ & Completeness to $\Theta_{\text {max }}$ & $79.2 \%{ }^{(a)}$ \\
Unit cell dimensions & $a=16.700(3) \AA$ & Max. and min. transmission & 0.7528 and 0.6427 \\
& $b=11.503(2) \AA$ & Data / restraints / parameters & $1723 / 2 / 252$ \\
& $c=10.836(3) \AA$ & Goodness-of-fit on $F^{2}$ & 1.040 \\
& $\alpha=90^{\circ}$ & Final $R$ indices $[I>2 \sigma(I)]$ & $R_{1}=0.0184, \mathrm{w} R_{2}=0.0433$ \\
& $\beta=90^{\circ}$ & $R$ indices (all data) & $R_{1}=0.0193, \mathrm{w} R_{2}=0.0436$ \\
& $\gamma=90^{\circ}$ & Absolute structure parameter & $0.008(11)$ \\
Volume & $2.0816(6) \mathrm{nm}^{3}$ & Extinction coefficient & $\mathrm{n} / \mathrm{a}$ \\
\hline & 4 & Largest diff. peak and hole & 0.105 and $-0.090 \mathrm{e} \AA^{-3}$ \\
\hline
\end{tabular}

(a): The low completeness of the data set is due to a blockage of the cooling system during the measurement along with the loss of the crystal. 


\subsection{5 $\left[(\mathrm{dmf}) \mathrm{ZnBr}\left\{\left(\mathrm{Me}_{2} \mathrm{NCH}_{2}\right)_{2} \mathrm{Acr}\right\}\right]\left[(\mathrm{dmf}) \mathrm{ZnBr}_{3}\right](18)$}

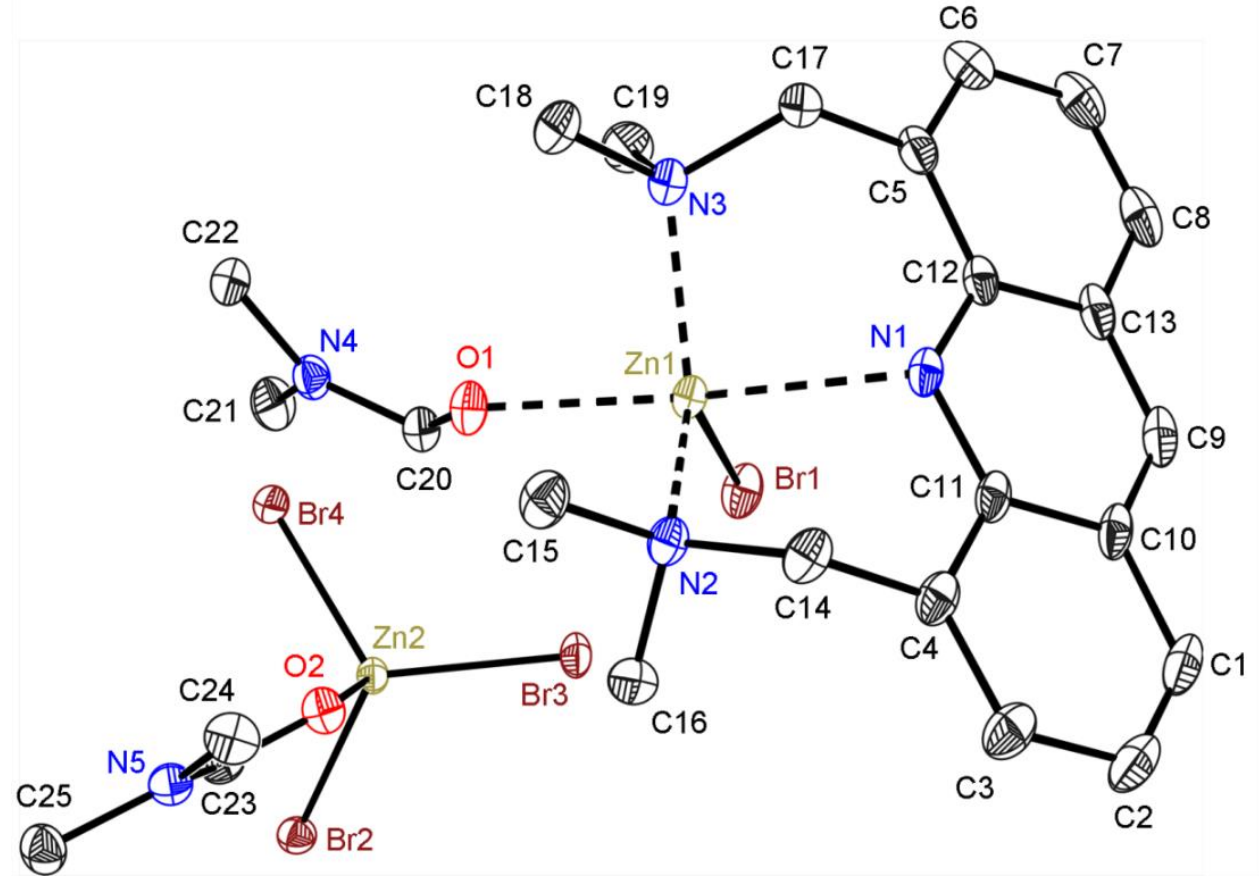

Figure 5.5: Solid state structure of $\mathbf{1 8}$. The anisotropic displacement parameters are depicted at the 50\% probability level. All hydrogen atoms are omitted for clarity. The structure is published in Dalton Trans. 2016, 45, 5689-5699. ${ }^{[111]}$

Table 5.5: Crystallographic information of $\mathbf{1 8}$.

\begin{tabular}{ll|ll}
\hline CCDC No. & 1061423 & Density (calculated) & $1.840 \mathrm{Mg} / \mathrm{m}^{3}$ \\
Structure code & $\mathrm{AcrNMe}_{2}-\mathrm{ZnDMF}$ & Absorption coefficient & $6.499 \mathrm{~mm}^{-1}$ \\
Empirical formula & $\mathrm{C}_{25} \mathrm{H}_{37} \mathrm{Br}_{4} \mathrm{~N}_{5} \mathrm{O}_{2} \mathrm{Zn} 2$ & $F(000)$ & 1752 \\
Formula weight & $889.97 \mathrm{~g} / \mathrm{mol}$ & Crystal size & $0.212 \times 0.179 \times 0.076 \mathrm{~mm}^{3}$ \\
Temperature & $100(2) \mathrm{K}$ & $\Theta$ range for data collection & 1.042 to $27.891^{\circ}$ \\
Wavelength & $0.71073 \AA$ & Reflections collected & 194567 \\
Crystal system & monoclinic & Independent reflections & $7674\left[R_{\text {int }}=0.0355\right]$ \\
Space group & $P{ }_{1} / c$ & Completeness to $\Theta_{\text {max }}$ & $99.9 \%$ \\
Unit cell dimensions & $a=20.985(3) \AA$ & Max. and min. transmission & 0.5631 and 0.3815 \\
& $b=9.749(2) \AA$ & Data / restraints / parameters & $7674 / 0 / 351$ \\
& $c=16.858(2) \AA$ & Goodness-of-fit on $F^{2}$ & 1.046 \\
& $\alpha=90^{\circ}$ & Final $R$ indices $[I>2 \sigma(I)]$ & $R_{1}=0.0204, \mathrm{w} R_{2}=0.0528$ \\
& $\beta=111.33(2)^{\circ}$ & $R$ indices (all data) & $R_{1}=0.0236, \mathrm{w} R_{2}=0.0542$ \\
Volume & $\gamma=90^{\circ}$ & Absolute structure parameter & $\mathrm{n} / \mathrm{a}$ \\
$Z$ & $3.2126(10) \mathrm{nm}^{3}$ & Extinction coefficient & $\mathrm{n} / \mathrm{a}$ \\
\hline
\end{tabular}




\subsection{6 $\left[\mathrm{CdBr}_{2}\left\{\left(\mathrm{Me}_{2} \mathrm{NCH}_{2}\right)_{2} \mathrm{Acr}\right\}\right](19)$}

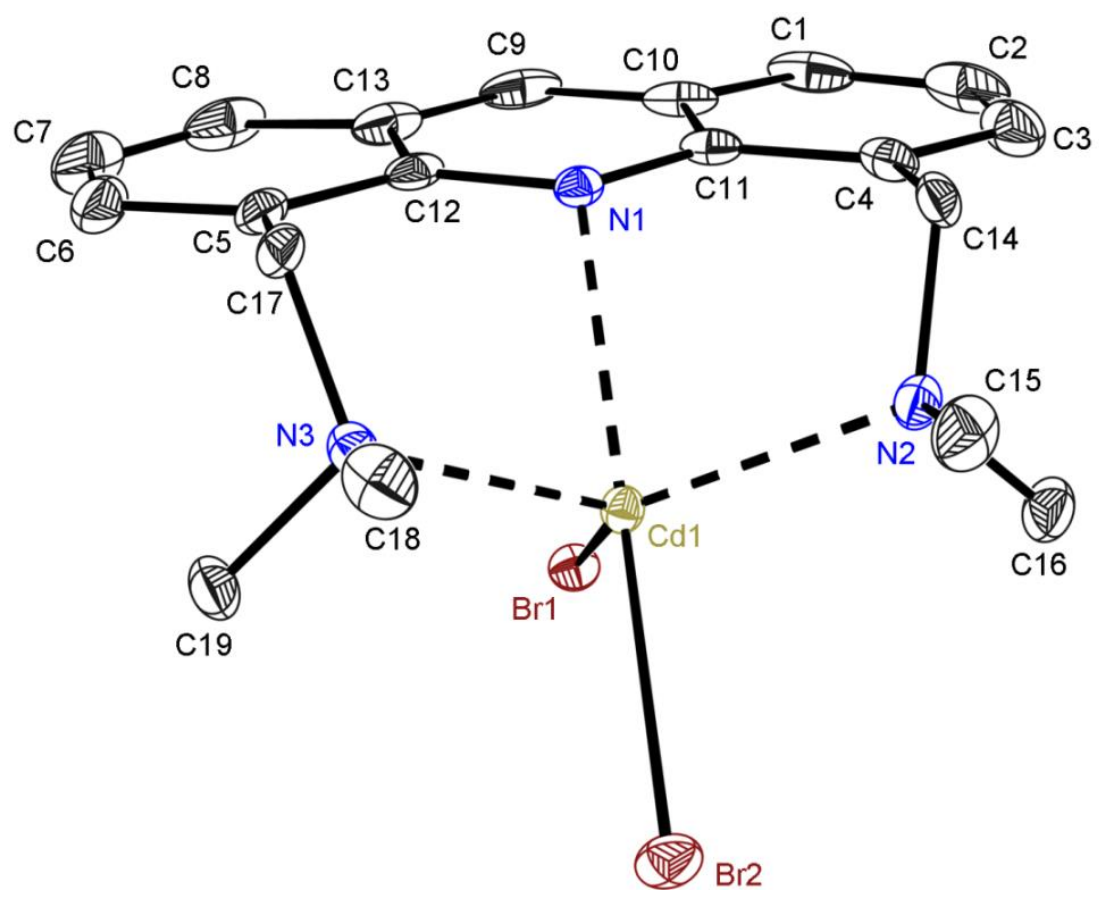

Figure 5.6: Solid state structure of 19. The anisotropic displacement parameters are depicted at the 50\% probability level. All hydrogen atoms are omitted for clarity. The structure is published in Dalton Trans. 2016, 45, 5689-5699. ${ }^{[11]}$

Table 5.6: Crystallographic information of 19.

\begin{tabular}{ll|ll}
\hline CCDC No. & 1061424 & Density (calculated) & $1.866 \mathrm{Mg} / \mathrm{m}^{3}$ \\
Structure code & $\mathrm{AcrNMe}_{2} \mathrm{Cd}$ & Absorption coefficient & $2.706 \mathrm{~mm}^{-1}$ \\
Empirical formula & $\mathrm{C}_{19} \mathrm{H}_{23} \mathrm{Br}_{2} \mathrm{CdN}_{3}$ & $F(000)$ & 1104 \\
Formula weight & $565.62 \mathrm{~g} / \mathrm{mol}$ & Crystal size & $0.225 \times 0.222 \times 0.185 \mathrm{~mm}^{3}$ \\
Temperature & $100(2) \mathrm{K}$ & $\Theta$ range for data collection & 1.690 to $21.684^{\circ}$ \\
Wavelength & $0.56086 \AA$ & Reflections collected & 77405 \\
Crystal system & orthorhombic & Independent reflections & $4810\left[R_{\text {int }}=0.0392\right]$ \\
Space group & $P 2_{1} 2_{1} 2_{1}$ & Completeness to $\Theta_{\text {max }}$ & $99.9 \%$ \\
Unit cell dimensions & $a=11.135(2) \AA$ & Max. and min. transmission & 0.7447 and 0.6799 \\
& $b=13.332(2) \AA$ & Data / restraints / parameters & $4810 / 0 / 231$ \\
& $c=13.562(3) \AA$ & Goodness-of-fit on $F^{2}$ & 1.014 \\
& $\alpha=90^{\circ}$ & Final $R$ indices $[I>2 \sigma(I)]$ & $R_{1}=0.0121, \mathrm{w} R_{2}=0.0261$ \\
& $\beta=90^{\circ}$ & $R$ indices (all data) & $R_{1}=0.0129, \mathrm{w} R_{2}=0.0263$ \\
Volume & $\gamma=90^{\circ}$ & Absolute structure parameter & $0.014(3)$ \\
$Z$ & $2.0133(6) \mathrm{nm}^{3}$ & Extinction coefficient & $0.00180(13)$ \\
\hline
\end{tabular}




\subsection{7 $\left[\mathrm{Zn}_{3}(\mu-\mathrm{OAc})_{4}(\mathrm{OAc})_{2}\left\{\left(\mathrm{Me}_{2} \mathrm{NCH}_{2}\right)_{2} \mathrm{Acr}\right\}\right](20)$}

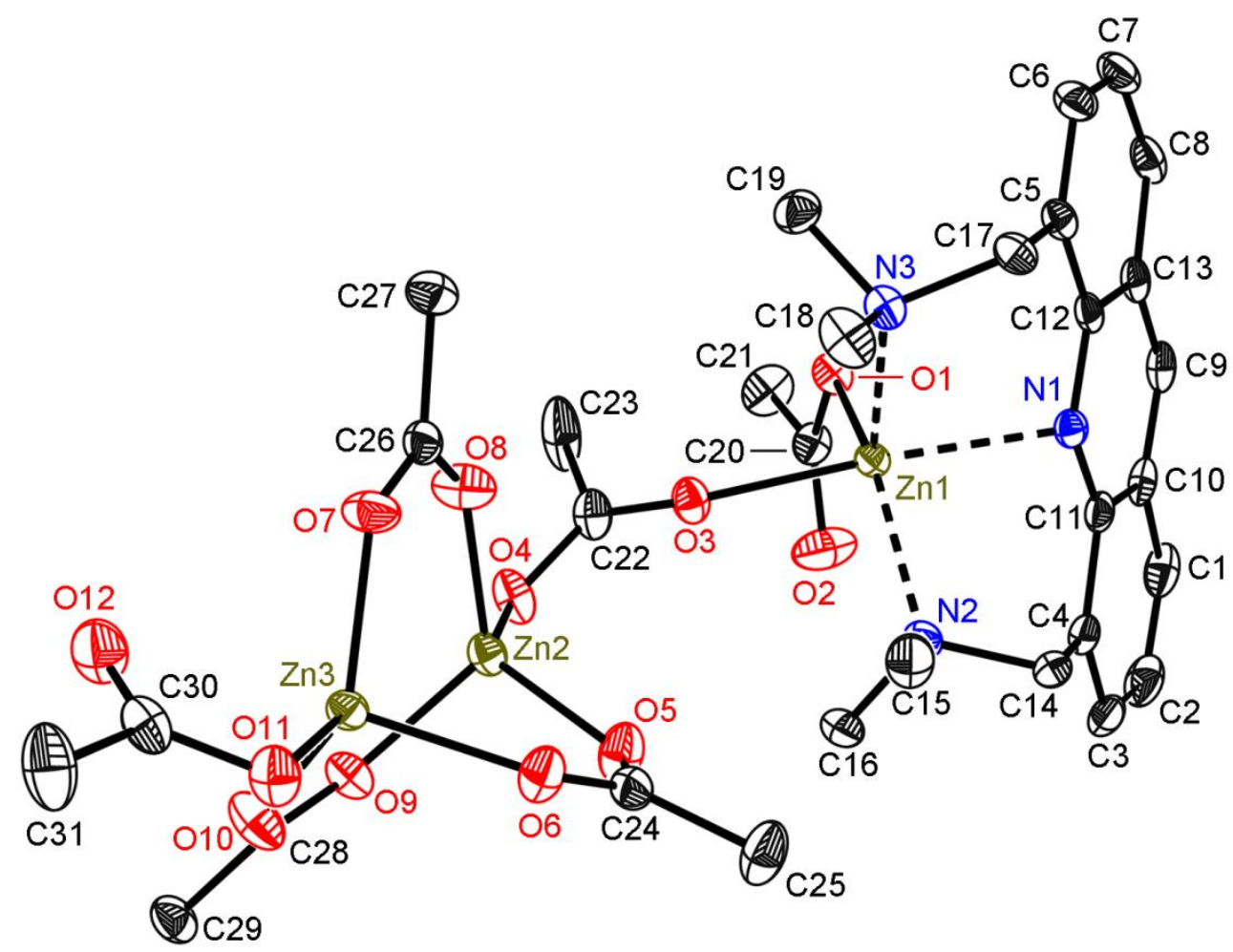

Figure 5.7: Solid state structure of 20. The anisotropic displacement parameters are depicted at the 50\% probability level. All hydrogen atoms are omitted for clarity. The structure is published in Dalton Trans. 2016, 45, 5689-5699..$^{[111]}$

Table 5.7: Crystallographic information of 20.

\begin{tabular}{ll|ll}
\hline CCDC No. & 1423218 & Density (calculated) & $1.602 \mathrm{Mg} / \mathrm{m}^{3}$ \\
Structure code & $\mathrm{AcrNMe}_{2} \mathrm{ZnOAc}$ & Absorption coefficient & $1.111 \mathrm{~mm}^{-1}$ \\
Empirical formula & $\mathrm{C}_{31} \mathrm{H}_{41} \mathrm{~N}_{3} \mathrm{O}_{12} \mathrm{Zn}_{3}$ & $F(000)$ & 1736 \\
Formula weight & $843.78 \mathrm{~g} / \mathrm{mol}$ & Crystal size & $0.179 \times 0.168 \times 0.122 \mathrm{~mm}^{3}$ \\
Temperature & $100(2) \mathrm{K}$ & $\Theta$ range for data collection & 1.940 to $20.553^{\circ}$ \\
Wavelength & $0.56086 \AA$ & Reflections collected & 178761 \\
Crystal system & monoclinic & Independent reflections & $7163\left[R_{\text {int }}=0.0485\right]$ \\
Space group & $P 2 / c$ & Completeness to $\Theta_{\text {max }}$ & $99.4 \%$ \\
Unit cell dimensions & $a=8.449(2) \AA$ & Max. and min. transmission & 0.5599 and 0.5243 \\
& $b=20.113(2) \AA$ & Data / restraints / parameters & $7163 / 0 / 452$ \\
& $c=21.008(3) \AA$ & Goodness-of-fit on $F^{2}$ & 1.037 \\
& $\alpha=90^{\circ}$ & Final $R$ indices $[I>2 \sigma(I)]$ & $R_{1}=0.0214, \mathrm{w}_{2}=0.0526$ \\
& $\beta=101.42(2)^{\circ}$ & $R$ indices (all data) & $R_{1}=0.0252, \mathrm{w} R_{2}=0.0544$ \\
& $\gamma=90^{\circ}$ & Absolute structure parameter & $\mathrm{n} / \mathrm{a}$ \\
Volume & $3.4993(11) \mathrm{nm}^{3}$ & Extinction coefficient & $\mathrm{n} / \mathrm{a}$ \\
\hline & 4 & Largest diff. peak and hole & 0.646 and $-0.458 \mathrm{e} \AA^{-3}$ \\
\hline
\end{tabular}




\subsection{8 $\left[\mathrm{Zn}_{2}(\mu-\mathrm{OMe})_{1.16} \mathrm{Br}_{2.84}\left\{\left(\mathrm{Me}_{2} \mathrm{NCH}_{2}\right)_{2} \mathrm{Acr}\right\}\right] \cdot($ thf $)(21)$}
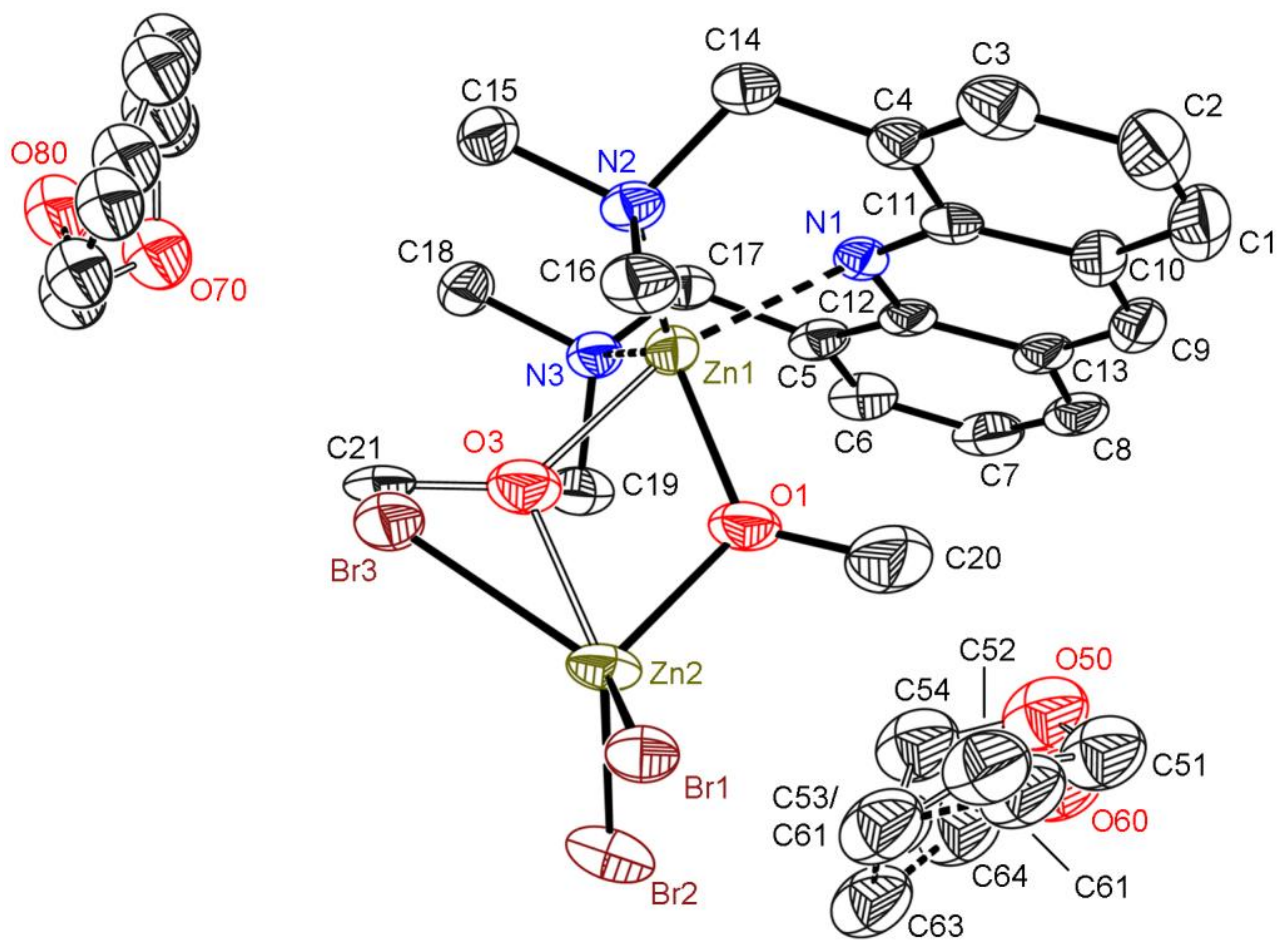

Figure 5.8: Solid state structure of 21. The anisotropic displacement parameters are depicted at the 50\% probability level. All hydrogen atoms are omitted for clarity. Both THF solvent molecules lie on an inversion centre and are disordered over two positions (sof: 0.277(6), 0.223(6), 0.244(8), 0.256(8)). The methoxide O3-C21 is disordered with $\mathrm{Br} 3$ (sof: 0.160(3) and 0.840(3)). The compound was formed during the attempt to crystallise $\mathrm{ZnBr}_{2}$ with 15 from a THF/MeOH mixture, whereby parts of the methanol solvent molecules were deprotonated.

Table 5.8: Crystallographic information of 21.

\begin{tabular}{ll|ll}
\hline CCDC No. & $\mathrm{n} / \mathrm{a}$ & Density (calculated) & $1.816 \mathrm{Mg} / \mathrm{m}^{3}$ \\
Structure code & AcrNMe2_ZnOMe & Absorption coefficient & $3.113 \mathrm{~mm}^{-1}$ \\
Empirical formula & $\mathrm{C}_{24.16} \mathrm{H}_{34.47} \mathrm{Br}_{2.84} \mathrm{~N}_{3}$ & $F(000)$ & 3017 \\
& $\mathrm{O}_{2.16} \mathrm{Zn}_{2}$ & & \\
Formula weight & 759.21 & Crystal size & $0.131 \times 0.094 \times 0.076 \mathrm{~mm}^{3}$ \\
Temperature & $100(2) \mathrm{K}$ & $\Theta$ range for data collection & 1.334 to $19.543^{\circ}$ \\
Wavelength & $0.56086 \AA$ & Reflections collected & 52354 \\
Crystal system & monoclinic & Independent reflections & $4945\left[R_{\text {int }}=0.0559\right]$ \\
Space group & $C 2 / c$ & Completeness to $\Theta_{\text {max }}$ & $99.8 \%$ \\
Unit cell dimensions & $a=16.197(2) \AA$ & Max. and min. transmission & 1.0000 and 0.8220 \\
& $b=18.066(2) \AA$ & Data / restraints / parameters & $4945 / 674 / 468$ \\
& $c=19.015(3) \AA$ & Goodness-of-fit on $F^{2}$ & 1.056 \\
& $\alpha=90^{\circ}$ & Final $R$ indices $[I>2 \sigma(I)]$ & $R_{1}=0.0386, \mathrm{w} R_{2}=0.0932$ \\
& $\beta=93.450(2)^{\circ}$ & $R$ indices (all data) & $R_{1}=0.0595, \mathrm{w} R_{2}=0.1055$ \\
$Z$ & $\gamma=90^{\circ}$ & Absolute structure parameter & $\mathrm{n} / \mathrm{a}$ \\
Volume & $5.5540(13) \mathrm{nm}^{3}$ & Extinction coefficient & $\mathrm{n} / \mathrm{a}$ \\
\hline
\end{tabular}




\subsection{9 $\left[\left(\mathrm{Me}_{2} \mathrm{NHCH}_{2}\right)_{2} \mathrm{Acr}\right] \mathrm{Br}_{2} \cdot(\mathrm{MeOH})(22)$}
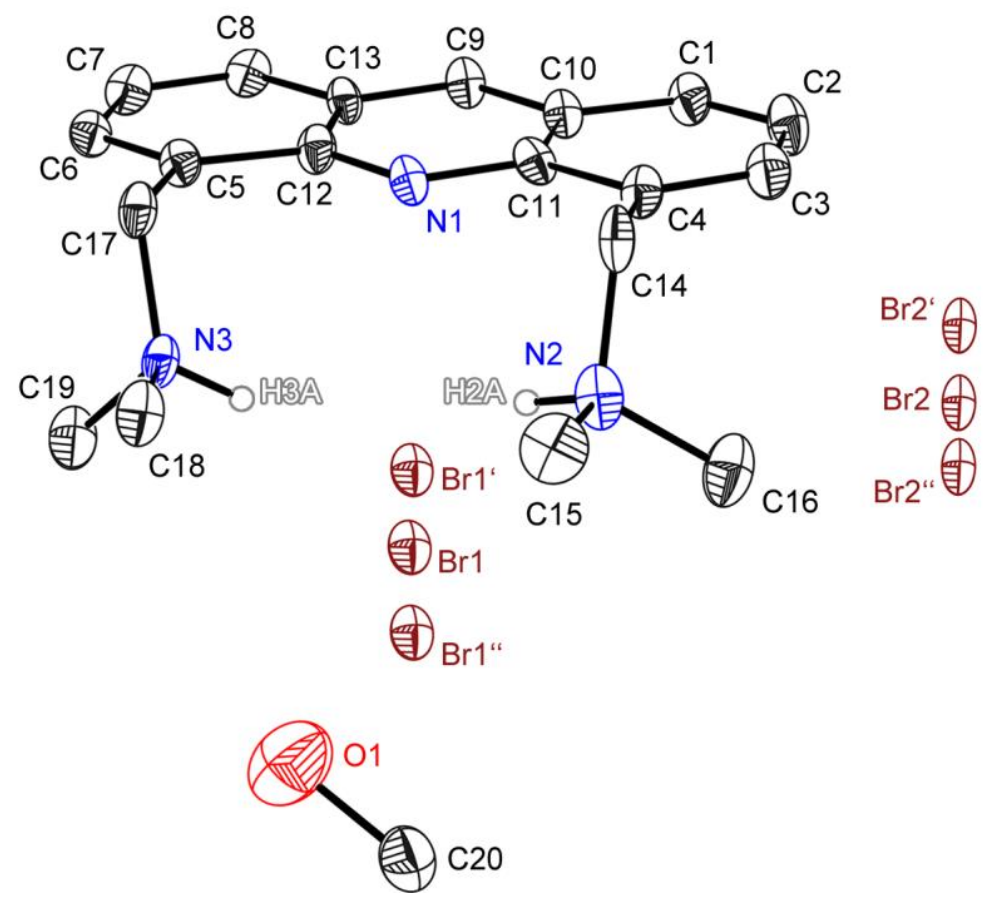

Figure 5.9: Solid state structure of 22. The anisotropic displacement parameters are depicted at the 50\% probability level. Not freely refined hydrogen atoms are omitted for clarity. Both bromide anions are disordered over three positions (sof: Br1: 0.941(2), 0.033(2), 0.026(2); Br2: 0.905(3), 0.074(2), 0.021(2)). The hydrogen atom at the oxygen (O1) could not be refined. The compound was formed during the attempt to crystallise $\mathrm{SnBr}_{2}$ with $\mathbf{1 5}$. Due to the Lewis acid properties of the metal salt, the ligand got protonated at its amines in a solution of methanol. The two bromide anions act as counter anions to the two-fold positive organic ligand.

Table 5.9: Crystallographic information of 22.

\begin{tabular}{ll|ll}
\hline CCDC No. & $\mathrm{n} / \mathrm{a}$ & Density (calculated) & $1.556 \mathrm{Mg} / \mathrm{m}^{3}$ \\
Structure code & AcrNMe2_SnBr2 & Absorption coefficient & $3.912 \mathrm{~mm}^{-1}$ \\
Empirical formula & $\mathrm{C}_{20} \mathrm{H}_{29} \mathrm{Br}_{2} \mathrm{~N}_{3} \mathrm{O}$ & $F(000)$ & 992 \\
Formula weight & 487.28 & Crystal size & $0.189 \times 0.114 \times 0.077 \mathrm{~mm}^{3}$ \\
Temperature & $100(2) \mathrm{K}$ & $\Theta$ range for data collection & 2.163 to $25.403^{\circ}$ \\
Wavelength & $0.71073 \AA$ & Reflections collected & 38418 \\
Crystal system & orthorhombic & Independent reflections & $3825\left[R_{\text {int }}=0.0640\right]$ \\
Space group & $P 2_{1} 2_{1} 2_{1}$ & Completeness to $\Theta_{\text {max }}$ & $100.0 \%$ \\
Unit cell dimensions & $a=11.135(2) \AA$ & Max. and min. transmission & 0.4288 and 0.3715 \\
& $b=11.608(2) \AA$ & Data / restraints / parameters & $3825 / 182 / 266$ \\
& $c=16.088(3) \AA$ & Goodness-of-fit on $F^{2}$ & 1.136 \\
& $\alpha=90^{\circ}$ & Final $R$ indices $[I>2 \sigma(I)]$ & $R_{1}=0.0435, \mathrm{w} R_{2}=0.0992$ \\
& $\beta=90^{\circ}$ & $R$ indices (all data) & $R_{1}=0.0517, \mathrm{w} R_{2}=0.1022$ \\
Volume & $\gamma=90^{\circ}$ & Absolute structure parameter & $0.50(3)$ \\
$Z$ & $2.0795(4) \mathrm{nm}^{3}$ & Extinction coefficient & $\mathrm{n} / \mathrm{a}$ \\
\hline
\end{tabular}




\section{CRYSTAL STRUCTURE DETERMINATION IN COLLABORATIONS}

\subsection{Structures determined for Mykyta Tretiakov (Prof. Dr. H. W. Roesky)}

\subsection{1 $\mathrm{I}_{2} \mathrm{~S}=\mathrm{C}\left[\mathrm{N}\left(2,6-{ }^{i} \mathrm{Pr}_{2}-\mathrm{C}_{6} \mathrm{H}_{3}\right) \mathrm{CH}\right]_{2}(23)$}

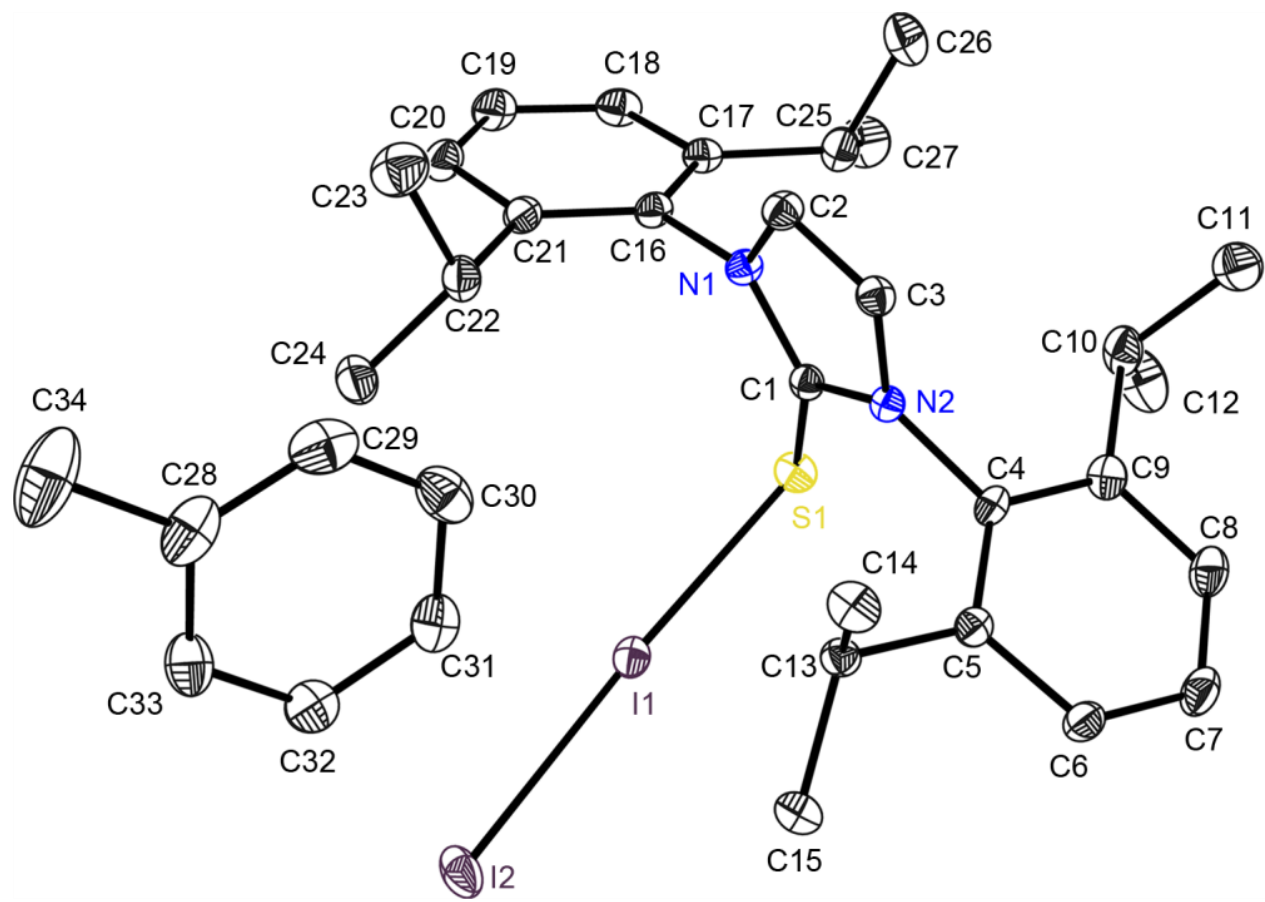

Figure 6.1: Solid state structure of $\mathbf{2 3}$. The anisotropic displacement parameters are depicted at the 50\% probability level. All hydrogen atoms are omitted for clarity. The structure is published in Dalton Transaction 2013, 42, 12940-12946. ${ }^{[168]}$

Table 6.1: Crystallographic information of 23.

\begin{tabular}{ll|ll}
\hline CCDC No. & 933418 & Density (calculated) & $1.494 \mathrm{Mg} / \mathrm{m}^{3}$ \\
Structure code & $\mathrm{AV} \_\mathrm{SN} 82$ & Absorption coefficient & $1.027 \mathrm{~mm}^{-1}$ \\
Empirical formula & $\mathrm{C}_{34} \mathrm{H}_{44} \mathrm{I}_{2} \mathrm{~N}_{2} \mathrm{~S}$ & $F(000)$ & 3072 \\
Formula weight & 766.57 & Crystal size & $0.178 \times 0.133 \times 0.063 \mathrm{~mm}^{3}$ \\
Temperature & $100(2) \mathrm{K}$ & $\Theta$ range for data collection & 0.867 to $23.633^{\circ}$ \\
Wavelength & $0.56086 \AA$ & Reflections collected & 103802 \\
Crystal system & monoclinic & Independent reflections & $10410\left[R_{\text {int }}=0.0635\right]$ \\
Space group & $C 2 / c$ & Completeness to $\Theta_{\max }$ & $100.0 \%$ \\
Unit cell dimensions & $a=37.684(3) \AA$ & Max. and min. transmission & 0.7449 and 0.6452 \\
& $b=11.414(2) \AA$ & Data / restraints / parameters & $10410 / 0 / 361$ \\
& $c=16.103(2) \AA$ & Goodness-of-fit on $F^{2}$ & 1.027 \\
& $\alpha=90.00^{\circ}$ & Final $R$ indices $[I>2 \sigma(I)]$ & $R_{1}=0.0304, \mathrm{w} R_{2}=0.0654$ \\
& $\beta=100.24(2)^{\circ}$ & $R$ indices (all data) & $R_{1}=0.0397, \mathrm{w} R_{2}=0.0687$ \\
Volume & $\gamma=90.00^{\circ}$ & Absolute structure parameter & $\mathrm{n} / \mathrm{a}$ \\
$\mathrm{Z}$ & $6816.0(16) \AA^{3}$ & Extinction coefficient & $\mathrm{n} / \mathrm{a}$ \\
\hline
\end{tabular}




\subsection{2 $\mathrm{I}_{2} \mathrm{~S}=\mathrm{C}\left(\mathrm{CH}_{2}\right)\left(\mathrm{CMe}_{2}\right)\left(\mathrm{C}_{6} \mathrm{H}_{10}\right) \mathrm{N}-2,6{ }^{i}{ }^{-} \mathrm{Pr}_{2}-\mathrm{C}_{6} \mathrm{H}_{3}(24)$}

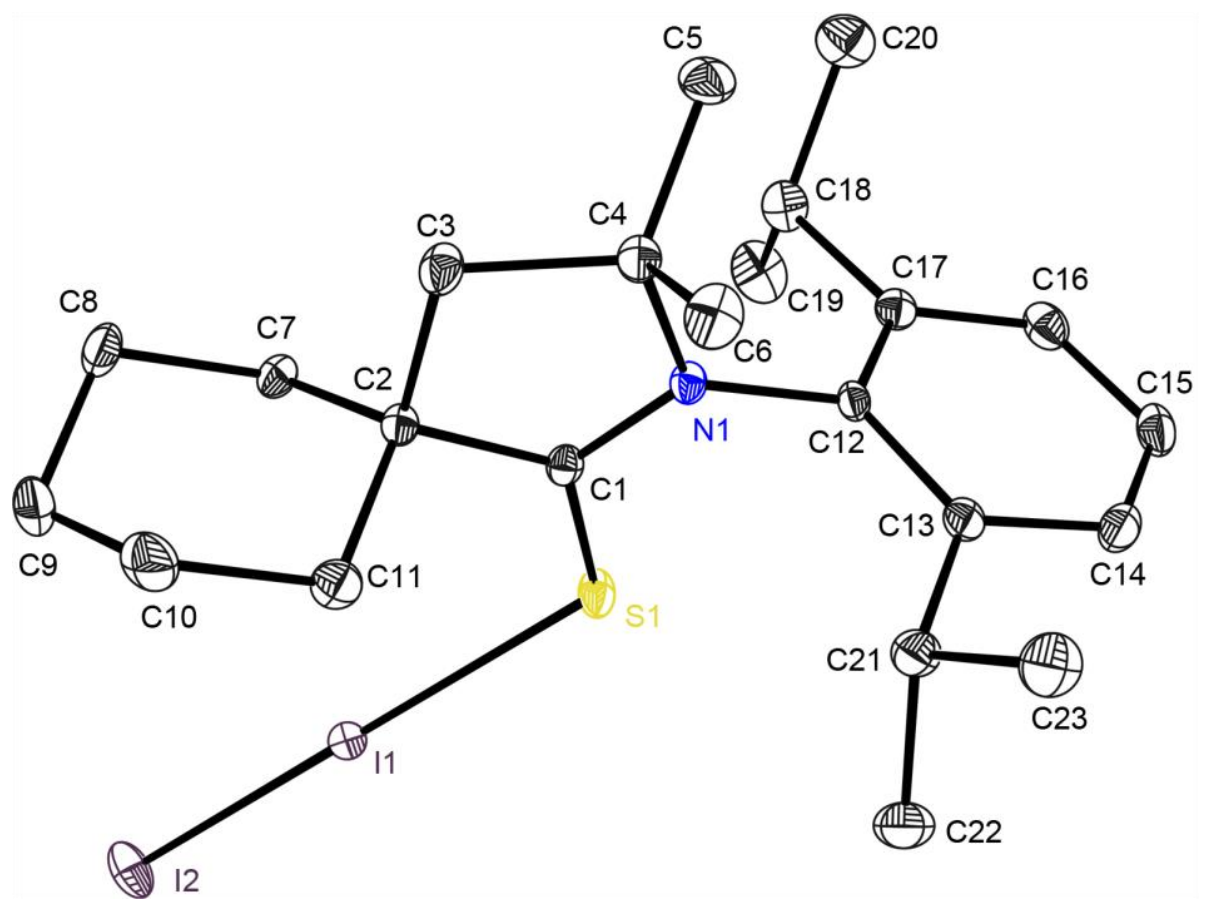

Figure 6.2: Solid state structure of $\mathbf{2 4}$. The anisotropic displacement parameters are depicted at the 50\% probability level. All hydrogen atoms are omitted for clarity. The structure is published in Dalton Transaction 2013, 42, 12940-12946. ${ }^{[168]}$

Table 6.2: Crystallographic information of $\mathbf{2 4}$.

\begin{tabular}{ll|ll}
\hline CCDC No. & 933419 & Density (calculated) & $1.657 \mathrm{Mg} / \mathrm{m}^{3}$ \\
Structure code & AV_SN84_1 & Absorption coefficient & $1.412 \mathrm{~mm}^{-1}$ \\
Empirical formula & $\mathrm{C}_{23} \mathrm{H}_{35} \mathrm{I}_{2} \mathrm{NS}$ & $F(000)$ & 604 \\
Formula weight & 611.38 & Crystal size & $0.118 \times 0.102 \times 0.088 \mathrm{~mm}^{3}$ \\
Temperature & $100(2) \mathrm{K}$ & $\Theta$ range for data collection & 1.351 to $25.679^{\circ}$ \\
Wavelength & $0.56086 \AA$ & Reflections collected & 50088 \\
Crystal system & triclinic & Independent reflections & $9450\left[R_{\text {int }}=0.0454\right]$ \\
Space group & $P \overline{1}$ & Completeness to $\Theta_{\text {max }}$ & $100.0 \%$ \\
Unit cell dimensions & $a=9.520(2) \AA$ & Max. and min. transmission & 1.0000 and 0.8996 \\
& $b=11.141(2) \AA$ & Data / restraints / parameters & $9450 / 0 / 250$ \\
& $c=12.138(2) \AA$ & Goodness-of-fit on $F^{2}$ & 1.054 \\
& $\alpha=91.69(2)^{\circ}$ & Final $R$ indices $[I>2 \sigma(I)]$ & $R_{1}=0.0255, \mathrm{w}_{2}=0.0527$ \\
& $\beta=100.62(2)^{\circ}$ & $R$ indices (all data) & $R_{1}=0.0357, \mathrm{w}_{2}=0.0552$ \\
Volume & $\gamma=103.70(2)^{\circ}$ & Absolute structure parameter & $\mathrm{n} / \mathrm{a}$ \\
$\mathrm{Z}$ & $1225.7(4) \AA^{3}$ & Extinction coefficient & $\mathrm{n} / \mathrm{a}$ \\
\hline
\end{tabular}




\subsection{3 $\mathrm{I}_{2} \mathrm{Te}=\mathrm{C}\left(\mathrm{CH}_{2}\right)\left(\mathrm{CMe}_{2}\right)_{2} \mathrm{~N}-2,6-{ }^{i} \mathrm{Pr}_{2}-\mathrm{C}_{6} \mathrm{H}_{3}(25)$}

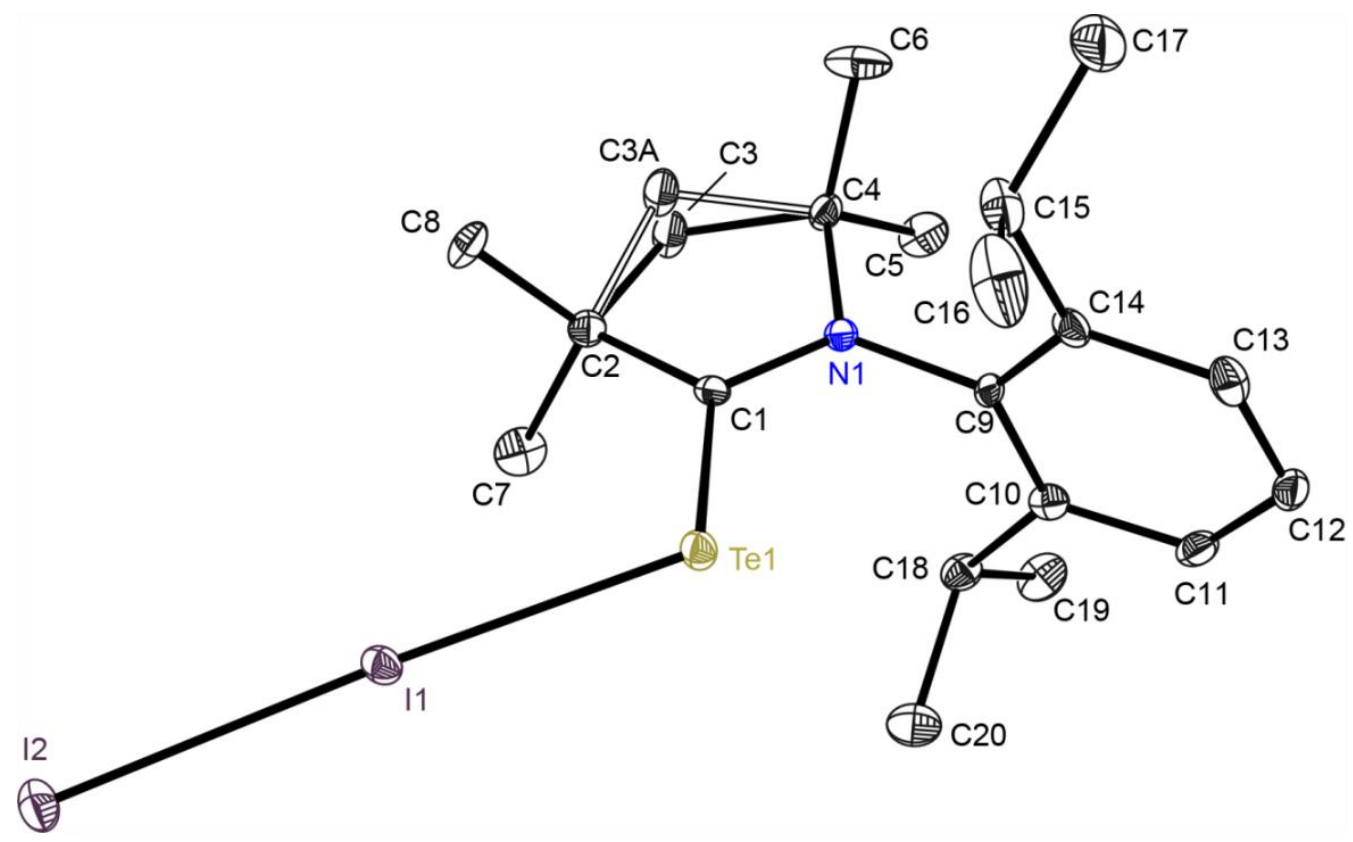

Figure 6.3: Solid state structure of 25. The anisotropic displacement parameters are depicted at the 50\% probability level. All hydrogen atoms are omitted for clarity. C3 is disordered over two positions (sof: 0.878(18)). The structure is published in Dalton Transaction 2013, 42, 12940-12946. ${ }^{[168]}$

Table 6.3: Crystallographic information of 25.

\begin{tabular}{ll|ll}
\hline CCDC No. & 933421 & Density (calculated) & $1.930 \mathrm{Mg} / \mathrm{m}^{3}$ \\
Structure code & $\mathrm{AV} \_\mathrm{SN} 94$ & Absorption coefficient & $2.116 \mathrm{~mm}^{-1}$ \\
Empirical formula & $\mathrm{C}_{20} \mathrm{H}_{31} \mathrm{I}_{2} \mathrm{NTe}$ & $F(000)$ & 1264 \\
Formula weight & 666.86 & Crystal size & $0.090 \times 0.085 \times 0.058 \mathrm{~mm}^{3}$ \\
Temperature & $100(2) \mathrm{K}$ & $\Theta$ range for data collection & 1.609 to $24.813^{\circ}$ \\
Wavelength & $0.56086 \AA$ & Reflections collected & 108910 \\
Crystal system & monoclinic & Independent reflections & $8016\left[R_{\text {int }}=0.0922\right]$ \\
Space group & $P 2_{1} / n$ & Completeness to $\Theta_{\max }$ & $100.0 \%$ \\
Unit cell dimensions & $a=11.108(2) \AA$ & Max. and min. transmission & 0.8140 and 0.7503 \\
& $b=16.440(2) \AA$ & Data / restraints / parameters & $8016 / 3 / 230$ \\
& $c=12.754(2) \AA$ & Goodness-of-fit on $F^{2}$ & 1.064 \\
& $\alpha=90.00^{\circ}$ & Final $R$ indices $[I>2 \sigma(I)]$ & $R_{1}=0.0313, \mathrm{w} R_{2}=0.0458$ \\
& $\beta=99.76(2)^{\circ}$ & $R$ indices (all data) & $R_{1}=0.0484, \mathrm{w}_{2}=0.0492$ \\
Volume & $\gamma=90.00^{\circ}$ & Absolute structure parameter & $\mathrm{n} / \mathrm{a}$ \\
$\mathrm{Z}$ & $2295.4(6) \AA^{3}$ & Extinction coefficient & $0.00047(6)$ \\
\hline
\end{tabular}




\subsection{Structures determined for Bastian Milde (Prof. Dr. D. B. Werz)}

6.2.1 Dispirane: $\mathrm{C}_{6} \mathrm{H}_{4} \mathrm{CCH}_{2} \mathrm{O}-\left(\mathrm{C}_{5} \mathrm{H}_{4}\left(\mathrm{CO}_{2} \mathrm{Me}\right)_{2}\right)_{2}-\mathrm{C}_{10} \mathrm{H}_{6} \mathrm{CCH}_{2} \mathrm{O}$ (26)

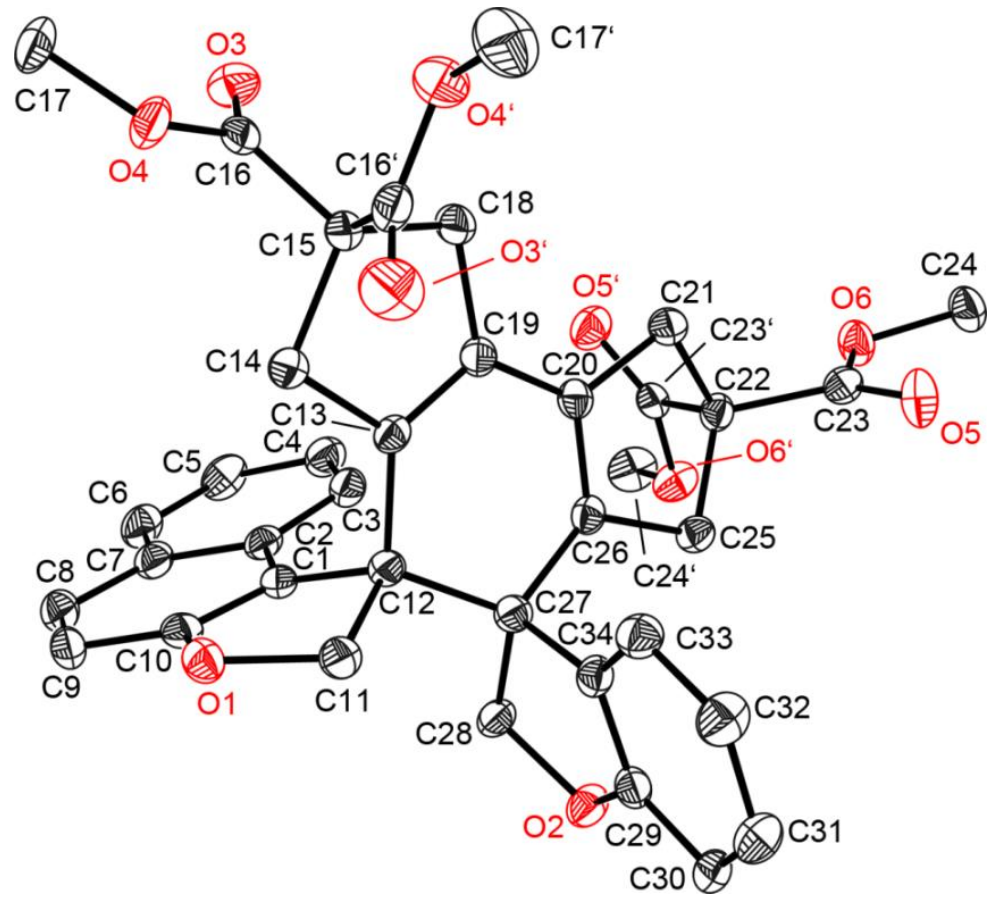

Figure 6.4: Solid state structure of 26. The anisotropic displacement parameters are depicted at the 50\% probability level. All hydrogen atoms are omitted for clarity. The structure is published in Chem.-Eur. J. 2015, 21, 16136-16146. ${ }^{[169]}$

Table 6.4: Crystallographic information of 26.

\begin{tabular}{ll|ll}
\hline CCDC No. & 1053289 & Density (calculated) & $1.364 \mathrm{Mg} / \mathrm{m}^{3}$ \\
Structure code & $\mathrm{BM} 2 \_04$ & Absorption coefficient & $0.099 \mathrm{~mm}^{-1}$ \\
Empirical formula & $\mathrm{C}_{38} \mathrm{H}_{34} \mathrm{O}_{10}$ & $F(000)$ & 1368 \\
Formula weight & 650.65 & Crystal size & $0.119 \times 0.117 \times 0.076 \mathrm{~mm}^{3}$ \\
Temperature & $100(2) \mathrm{K}$ & $\Theta$ range for data collection & 1.263 to $26.385^{\circ}$ \\
Wavelength & $0.71073 \AA$ & Reflections collected & 38399 \\
Crystal system & orthorhombic & Independent reflections & $6496\left[R_{\text {int }}=0.0482\right]$ \\
Space group & $P 2_{1} 2_{1} 2_{1}$ & Completeness to $\Theta_{\text {max }}$ & $99.9 \%$ \\
Unit cell dimensions & $a=9.221(2) \AA$ & Max. and min. transmission & 0.7446 and 0.7319 \\
& $b=10.655(2) \AA$ & Data / restraints / parameters & $6496 / 0 / 437$ \\
& $c=32.257(2) \AA$ & Goodness-of-fit on $F^{2}$ & 1.080 \\
& $\alpha=90.00^{\circ}$ & Final $R$ indices $[I>2 \sigma(I)]$ & $R_{1}=0.0376, \mathrm{w}_{2}=0.0893$ \\
& $\beta=90.00^{\circ}$ & $R$ indices (all data) & $R_{1}=0.0450, \mathrm{w} R_{2}=0.0925$ \\
Volume & $\gamma=90.00^{\circ}$ & Absolute structure parameter & $0.0(3)$ \\
$\mathrm{Z}$ & $3.1692(9) \mathrm{nm}^{3}$ & Extinction coefficient & $\mathrm{n} / \mathrm{a}$ \\
\hline
\end{tabular}




\subsubsection{Dispirane: $\mathrm{C}_{12} \mathrm{H}_{12}\left(\mathrm{CO}_{2} \mathrm{Me}\right)_{4}\left(\mathrm{CH}_{2} \mathrm{OC}_{6} \mathrm{H}_{3}\left(\mathrm{CO}_{2} \mathrm{Me}\right)\right)_{2}(27)$}

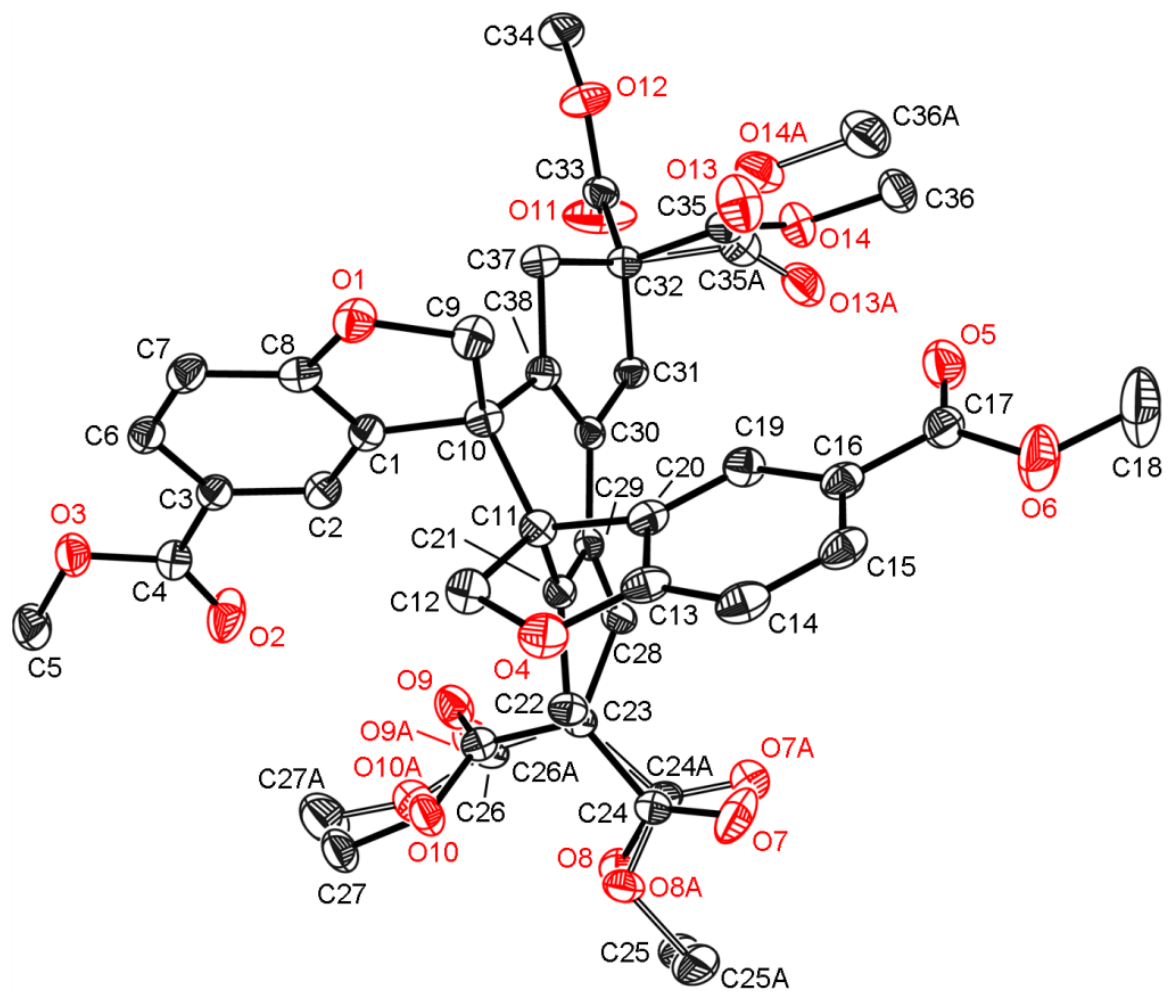

Figure 6.5: Solid state structure of 27. The anisotropic displacement parameters are depicted at the 50\% probability level. All hydrogen atoms are omitted for clarity. The ester groups at C22, C23, and C32 are disordered over two positions (sof: 0.883(17), 0.763(14), and 0.935(4), respectively). The structure is published in Chem.-Eur. J. 2015, 21, 16136-16146. ${ }^{[169]}$

Table 6.5: Crystallographic information of 27.

\begin{tabular}{ll|ll}
\hline CCDC No. & 1053290 & Density (calculated) & $1.436 \mathrm{Mg} / \mathrm{m}^{3}$ \\
Structure code & BM2_871b & Absorption coefficient & $0.929 \mathrm{~mm}^{-1}$ \\
Empirical formula & C38 H36 O14 & $F(000)$ & 1504 \\
Formula weight & 716.67 & Crystal size & $0.09 \times 0.07 \times 0.02 \mathrm{~mm}^{3}$ \\
Temperature & $100(2) \mathrm{K}$ & $\Theta$ range for data collection & 5.541 to $70.071^{\circ}$ \\
Wavelength & $1.54178 \AA$ & Reflections collected & 91199 \\
Crystal system & orthorhombic & Independent reflections & $6147\left[R_{\text {int }}=0.0388\right]$ \\
Space group & $P c a 2_{1}$ & Completeness to $\Theta_{\text {max }}$ & $98.1 \%$ \\
Unit cell dimensions & $a=17.674(2) \AA$ & Max. and min. transmission & 0.8644 and 0.7943 \\
& $b=7.983(2) \AA$ & Data / restraints / parameters & $6147 / 639 / 587$ \\
& $c=23.492(2) \AA$ & Goodness-of-fit on $F^{2}$ & 1.079 \\
& $\alpha=90.00^{\circ}$ & Final $R$ indices $[I>2 \sigma(I)]$ & $R_{1}=0.0289, \mathrm{w} R_{2}=0.0798$ \\
& $\beta=90.00^{\circ}$ & $R$ indices (all data) & $R_{1}=0.0295, \mathrm{w} R_{2}=0.0800$ \\
Volume & $\gamma=90.00^{\circ}$ & Absolute structure parameter & $0.38(2)$ \\
$\mathrm{Z}$ & $3314.5(10) \AA^{3}$ & Extinction coefficient & $\mathrm{n} / \mathrm{a}$ \\
\hline
\end{tabular}




\subsection{Structures determined for Tobias Schneider (Prof. Dr. D. B. Werz)}

\subsubsection{Spiro[anthracene-9(10H), $1^{\prime}\left(3^{\prime} H\right)$-isobenzofuran]-3'10-dione (28)}

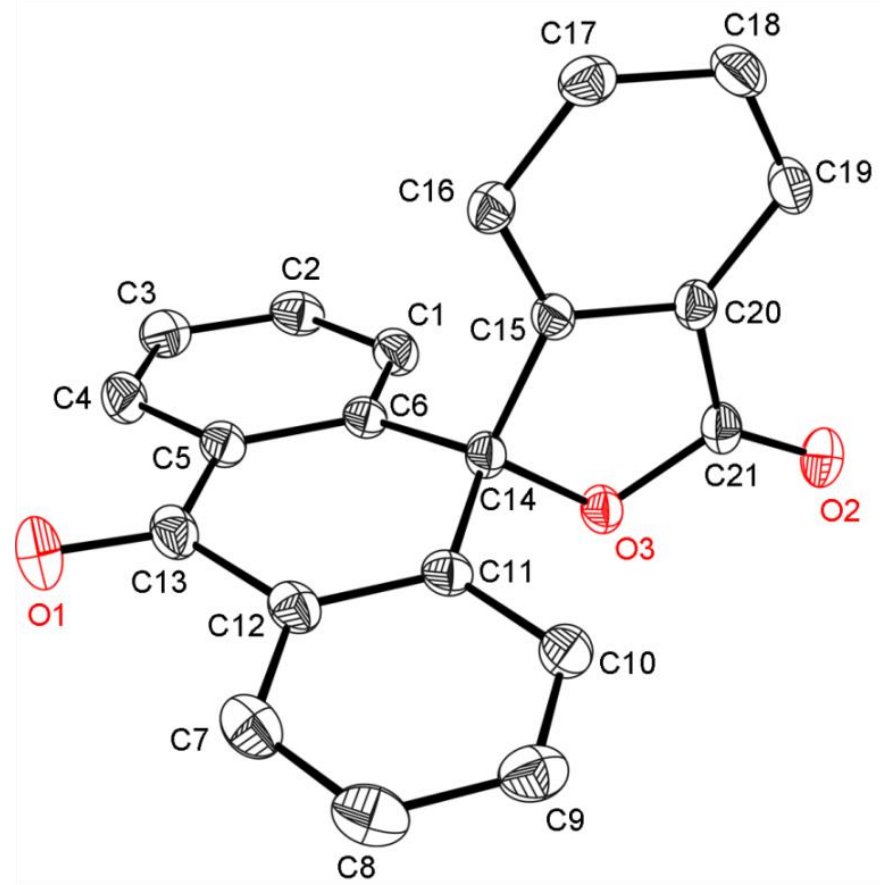

Figure 6.6: Solid state structure of $\mathbf{2 8}$. The anisotropic displacement parameters are depicted at the $50 \%$ probability level. All hydrogen atoms are omitted for clarity.

Table 6.6: Crystallographic information of 28.

\begin{tabular}{ll|ll}
\hline CCDC No. & $\mathrm{n} / \mathrm{a}$ & Density (calculated) & $1.449 \mathrm{Mg} / \mathrm{m}^{3}$ \\
Structure code & $\mathrm{ST}_{2} 01$ & Absorption coefficient & $0.097 \mathrm{~mm}^{-1}$ \\
Empirical formula & $\mathrm{C}_{21} \mathrm{H}_{12} \mathrm{O}_{3}$ & $F(000)$ & 324 \\
Formula weight & 312.31 & Crystal size & $0.182 \times 0.125 \times 0.057 \mathrm{~mm}^{3}$ \\
Temperature & $100(2) \mathrm{K}$ & $\Theta$ range for data collection & 1.974 to $25.349^{\circ}$ \\
Wavelength & $0.71073 \AA$ & Reflections collected & 11019 \\
Crystal system & triclinic & Independent reflections & $2617\left[R_{\text {int }}=0.0238\right]$ \\
Space group & $P \overline{1}$ & Completeness to $\Theta_{\text {max }}$ & $99.9 \%$ \\
Unit cell dimensions & $a=8.635(2) \AA$ & Max. and min. transmission & 1.0000 and 0.9285 \\
& $b=9.075(2) \AA$ & Data / restraints / parameters & $2617 / 0 / 217$ \\
& $c=10.553(2) \AA$ & Goodness-of-fit on $F^{2}$ & 1.038 \\
& $\alpha=78.25(2)^{\circ}$ & Final $R$ indices $[I>2 \sigma(I)]$ & $R_{1}=0.0338, \mathrm{w} R_{2}=0.0881$ \\
& $\beta=81.78(2)^{\circ}$ & $R$ indices (all data) & $R_{1}=0.0386, \mathrm{w} R_{2}=0.0910$ \\
Volume & $\gamma=62.31(2)^{\circ}$ & Absolute structure parameter & $\mathrm{n} / \mathrm{a}$ \\
$\mathrm{Z}$ & $715.8(3) \AA^{3}$ & Extinction coefficient & $\mathrm{n} / \mathrm{a}$ \\
\hline
\end{tabular}




\subsubsection{0,15-Dihydro-5 $H$-tribenzo $[a, d, g]$ cyclononen-5-ol (29)}

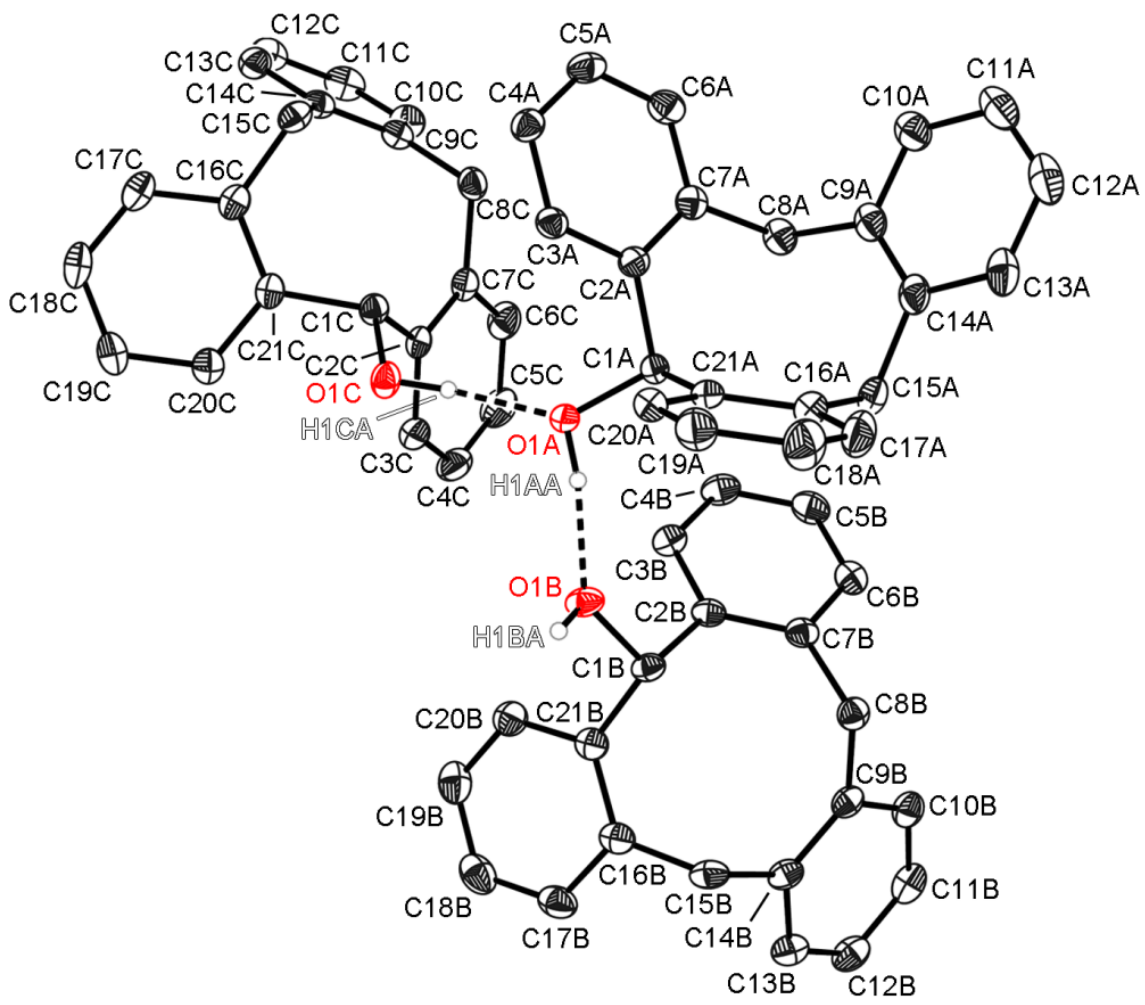

Figure 6.7: Solid state structure of 29. The anisotropic displacement parameters are depicted at the 50\% probability level. Not freely refined hydrogen atoms are omitted for clarity.

Table 6.7: Crystallographic information of 29.

\begin{tabular}{ll|ll}
\hline CCDC No. & $\mathrm{n} / \mathrm{a}$ & Density (calculated) & $1.204 \mathrm{Mg} / \mathrm{m}^{3}$ \\
Identification code & $\mathrm{ST} 4-\mathrm{X} 13$ & Absorption coefficient & $0.072 \mathrm{~mm}^{-3}$ \\
Empirical formula & $\mathrm{C}_{63} \mathrm{H}_{54} \mathrm{O}_{3}$ & $F(000)$ & 1824 \\
Formula weight & 859.06 & Crystal size & $0.408 \times 0.300 \times 0.072 \mathrm{~mm}^{3}$ \\
Temperature & $100(2) \mathrm{K}$ & $\Theta$ range for data collection & 1.606 to $27.282^{\circ}$ \\
Wavelength & $0.71073 \AA$ & Reflections collected & 87786 \\
Crystal system & monoclinic & Independent reflections & $10546\left[R_{\text {int }}=0.0332\right]$ \\
Space group & $P{ }_{1} / n$ & Completeness to $\Theta_{\max }$ & $100.0 \%$ \\
Unit cell dimensions & $a=16.786(2) \AA$ & Max. and min. transmission & 1.0000 and 0.9490 \\
& $b=16.518(3) \AA$ & Data / restraints / parameters & $10546 / 3 / 605$ \\
& $c=17.202(2) \AA$ & Goodness-of-fit on $F^{2}$ & 1.062 \\
& $\alpha=90.00^{\circ}$ & Final $R$ indices $[I>2 \sigma(I)]$ & $R_{1}=0.0404, \mathrm{w} R_{2}=0.0999$ \\
& $\beta=96.58(2)^{\circ}$ & $R$ indices (all data) & $R_{1}=0.0466, \mathrm{w} R_{2}=0.1033$ \\
Volume & $\gamma=90.00^{\circ}$ & Absolute structure parameter & $\mathrm{n} / \mathrm{a}$ \\
$\mathrm{Z}$ & $4.7382(12) \mathrm{nm}^{3}$ & Extinction coefficient & $0.0022(2)$ \\
\hline & 4 & Largest diff. peak and hole & 0.287 and $-0.217 \mathrm{e} \AA^{-3}$ \\
\hline
\end{tabular}




\subsection{Structures determined for Matheus Bukala}

\subsection{1 $\left[\mathrm{Ph}_{2} \mathrm{SP}\left(\mathrm{C}_{14} \mathrm{H}_{8}\right) \mathrm{CH}_{2} \mathrm{NH}\left(\left(\mathrm{CH}_{2}\right)_{2} \mathrm{OCH}_{3}\right)_{2}\right]^{+}\left[(\text {thf }) \mathrm{ZnBr}_{3}\right]^{-}(30)$}

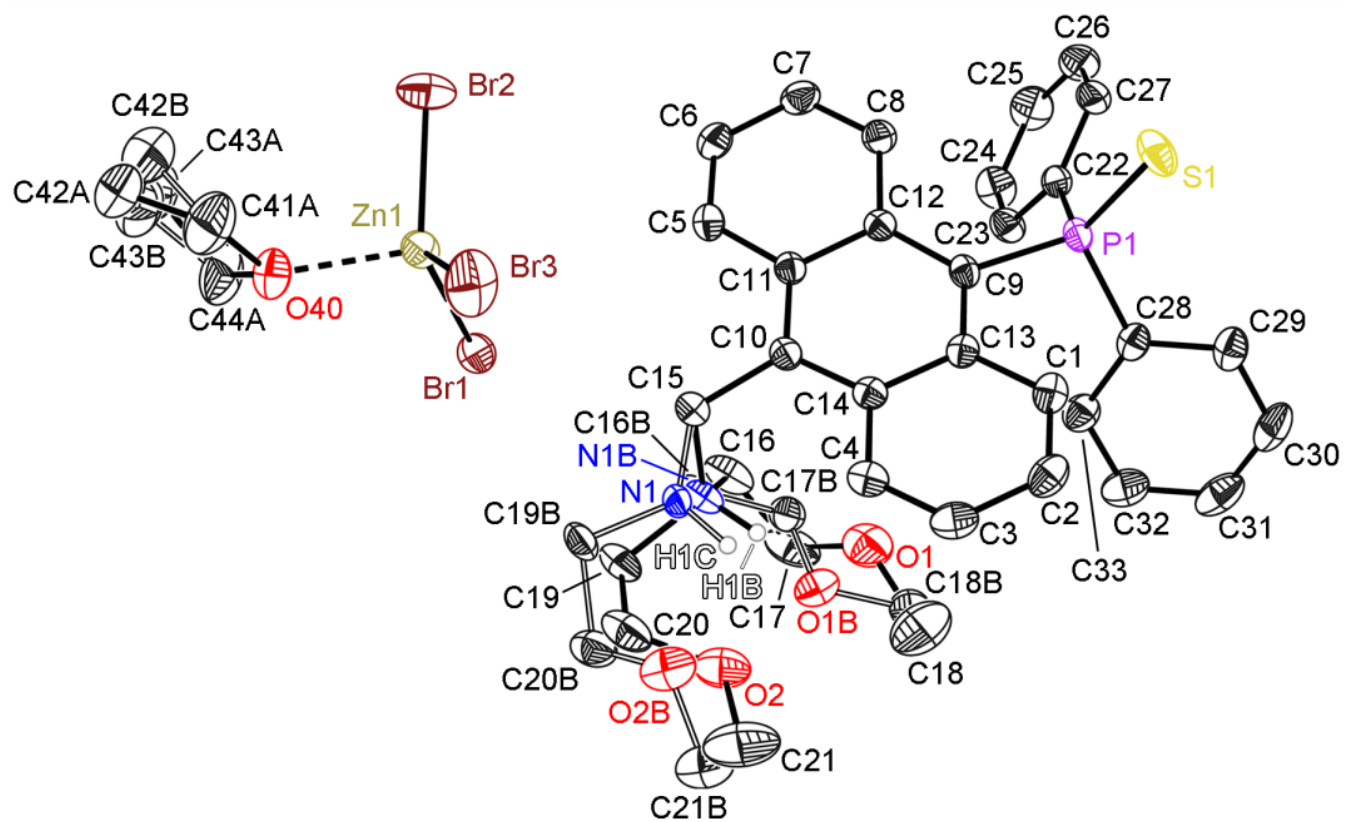

Figure 6.8: Solid state structure of 30. The anisotropic displacement parameters are depicted at the 50\% probability level. Not freely refined hydrogen atoms are omitted for clarity. The amine group (N1) and the THF molecule are disordered over two positions (sof: 0.897(4) and 0.742(21), respectively).

Table 6.8: Crystallographic information of $\mathbf{3 0}$.

\begin{tabular}{ll|ll}
\hline CCDC No. & $\mathrm{n} / \mathrm{a}$ & Density (calculated) & $1.554 \mathrm{Mg} / \mathrm{m}^{3}$ \\
Structure code & SPAnAmin_Zn & Absorption coefficient & $2.035 \mathrm{~mm}^{-1}$ \\
Empirical formula & $\mathrm{C}_{37} \mathrm{H}_{43} \mathrm{Br}_{3} \mathrm{NO}_{3} \mathrm{PSZn}$ & $F(000)$ & 924 \\
Formula weight & 917.85 & Crystal size & $0.425 \times 0.319 \times 0.269 \mathrm{~mm}^{3}$ \\
Temperature & $170(2) \mathrm{K}$ & $\Theta$ range for data collection & 1.531 to $20.594^{\circ}$ \\
Wavelength & $0.56086 \AA$ & Reflections collected & 71266 \\
Crystal system & triclinic & Independent reflections & $8086\left[R_{\text {int }}=0.0223\right]$ \\
Space group & $P \overline{1}$ & Completeness to $\Theta_{\text {max }}$ & $100.0 \%$ \\
Unit cell dimensions & $a=10.544(2) \AA$ & Max. and min. transmission & 0.7445 and 0.6315 \\
& $b=13.126(2) \AA$ & Data / restraints / parameters & $8086 / 584 / 531$ \\
& $c=15.345(3) \AA$ & Goodness-of-fit on $F^{2}$ & 1.024 \\
& $\alpha=79.08(2)^{\circ}$ & Final $R$ indices $[I>2 \sigma(I)]$ & $R_{1}=0.0289, \mathrm{w} R_{2}=0.0679$ \\
& $\beta=70.18(2)^{\circ}$ & $R$ indices (all data) & $R_{1}=0.0337, \mathrm{w} R_{2}=0.0713$ \\
Volume & $\gamma=85.37(3)^{\circ}$ & Absolute structure parameter & $\mathrm{n} / \mathrm{a}$ \\
$\mathrm{Z}$ & $1.9615(7) \mathrm{nm}^{3}$ & Extinction coefficient & $\mathrm{n} / \mathrm{a}$ \\
\hline
\end{tabular}




\subsection{Structures determined for Lianrong Liu}

\subsubsection{9,9'-Ethenediyldiacridine (31)}

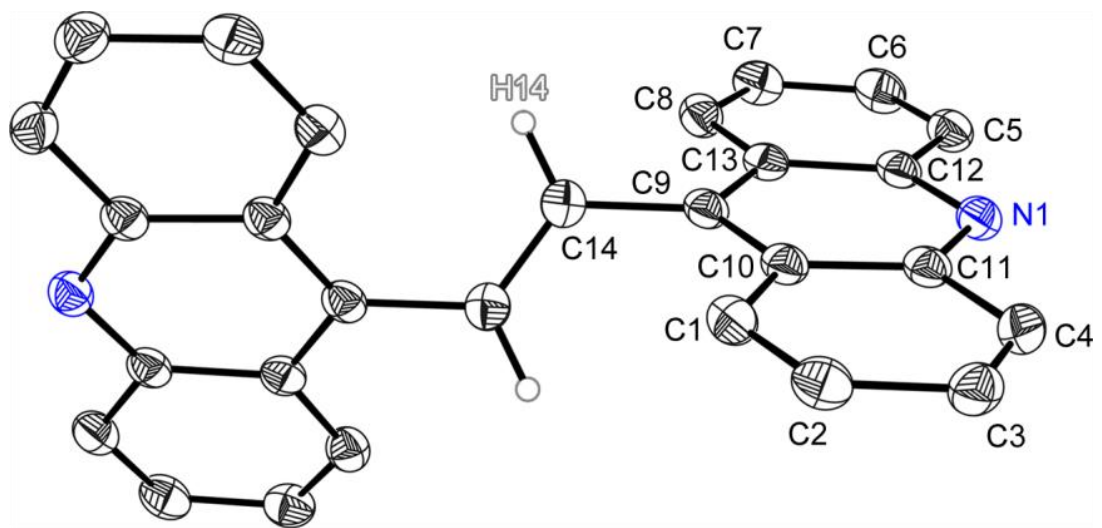

Figure 6.9: Solid state structure of 31. The anisotropic displacement parameters are depicted at the 50\% probability level. The hydrogen atoms at the aromatic system have been omitted for clarity. Only the half of the molecule is present in the asymmetric unit. The other half is generated by a symmetry operation (1- $\mathrm{x}$, $+\mathrm{y}, 3 / 2-\mathrm{z})$.

Table 6.9: Crystallographic information of $\mathbf{3 1}$.

\begin{tabular}{ll|ll}
\hline CCDC No. & $\mathrm{n} / \mathrm{a}$ & Density (calculated) & $1.384 \mathrm{Mg} / \mathrm{m}^{3}$ \\
Structure code & $\mathrm{LL} 14$ & Absorption coefficient & $0.081 \mathrm{~mm}^{-1}$ \\
Empirical formula & $\mathrm{C}_{28} \mathrm{H}_{18} \mathrm{~N}_{2}$ & $F(000)$ & 800 \\
Formula weight & 382.44 & Crystal size & $0.350 \times 0.256 \times 0.146 \mathrm{~mm}^{3}$ \\
Temperature & $100(2) \mathrm{K}$ & $\Theta$ range for data collection & 2.393 to $25.367^{\circ}$ \\
Wavelength & $0.71073 \AA$ & Reflections collected & 71266 \\
Crystal system & monoclinic & Independent reflections & $1674\left[R_{\mathrm{int}}=0.0256\right]$ \\
Space group & $C 2 / c$ & Completeness to $\Theta_{\max }$ & $99.9 \%$ \\
Unit cell dimensions & $a=13.550(3) \AA$ & Max. and min. transmission & $0.7452 \mathrm{and} 0.7091$ \\
& $b=12.211(2) \AA$ & Data / restraints / parameters & $1674 / 0 / 136$ \\
& $c=12.660(2) \AA$ & Goodness-of-fit on $F^{2}$ & 1.075 \\
& $\alpha=90^{\circ}$ & Final $R$ indices $[I>2 \sigma(I)]$ & $R_{1}=0.0387, \mathrm{w} R_{2}=0.1049$ \\
& $\beta=118.83(2)^{\circ}$ & $R$ indices (all data) & $R_{1}=0.0420, \mathrm{w} R_{2}=0.1083$ \\
& $\gamma=90^{\circ}$ & Absolute structure parameter & $\mathrm{n} / \mathrm{a}$ \\
Volume & $1.8351(7) \mathrm{nm}^{3}$ & Extinction coefficient & $\mathrm{n} / \mathrm{a}$ \\
$Z$ & 4 & Largest diff. peak and hole & $0.263 \mathrm{and}-0.188 \mathrm{e} \AA^{-3}$ \\
\hline
\end{tabular}




\subsubsection{Acridine-10-oxide $\cdot 2 \mathrm{H}_{2} \mathrm{O}(32)$}

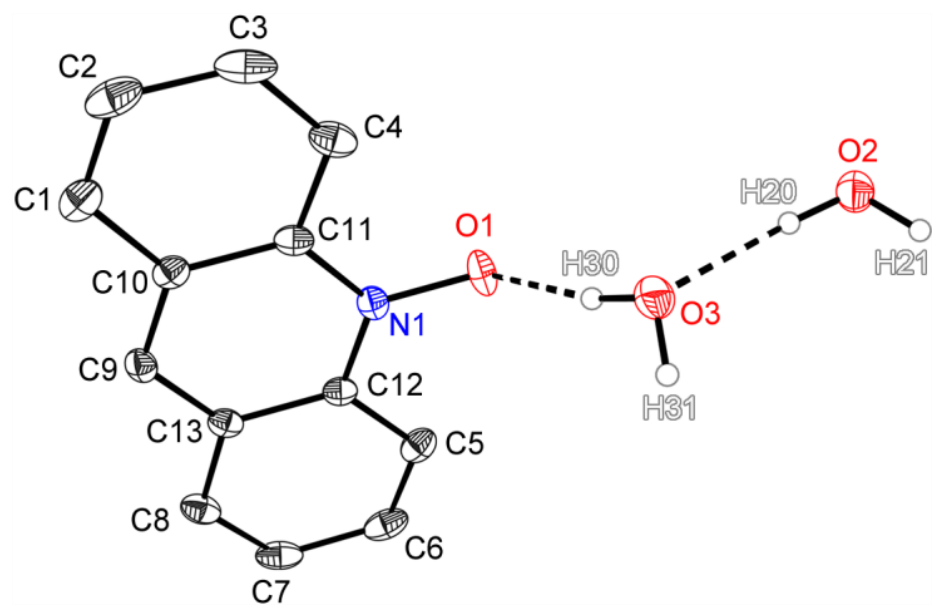

Figure 6.10: Solid state structure of 32. The anisotropic displacement parameters are depicted at the $50 \%$ probability level. Not freely refined hydrogen atoms have been omitted for clarity.

Table 6.10: Crystallographic information of $\mathbf{3 2}$.

\begin{tabular}{ll|ll}
\hline CCDC No. & $\mathrm{n} / \mathrm{a}$ & Density (calculated) & $1.385 \mathrm{Mg} / \mathrm{m}^{3}$ \\
Structure code & $\mathrm{LL17}$ & Absorption coefficient & $0.062 \mathrm{~mm}^{-1}$ \\
Empirical formula & $\mathrm{C}_{13} \mathrm{H}_{13} \mathrm{NO}_{3}$ & $F(000)$ & 488 \\
Formula weight & 231.24 & Crystal size & $0.529 \times 0.332 \times 0.156 \mathrm{~mm}^{3}$ \\
Temperature & $100(2) \mathrm{K}$ & $\Theta$ range for data collection & 1.707 to $20.308^{\circ}$ \\
Wavelength & $0.56086 \AA$ & Reflections collected & 44220 \\
Crystal system & monoclinic & Independent reflections & $2198\left[R_{\text {int }}=0.0434\right]$ \\
Space group & $P 2{ }_{1} / n$ & Completeness to $\Theta$ max & $99.9 \%$ \\
Unit cell dimensions & $a=11.565(3) \AA$ & Max. and min. transmission & 0.7445 and 0.6561 \\
& $b=6.762(2) \AA$ & Data / restraints / parameters & $2198 / 6 / 171$ \\
& $c=14.254(3) \AA$ & Goodness-of-fit on $F^{2}$ & 1.070 \\
& $\alpha=90^{\circ}$ & Final $R$ indices $[I>2 \sigma(I)]$ & $R_{1}=0.0375, \mathrm{w} R_{2}=0.1104$ \\
& $\beta=95.83(2)^{\circ}$ & $R$ indices (all data) & $R_{1}=0.0437, \mathrm{w} R_{2}=0.1155$ \\
& $\gamma=90^{\circ}$ & Absolute structure parameter & $\mathrm{n} / \mathrm{a}$ \\
Volume & $1.1089(5) \mathrm{nm}^{3}$ & Extinction coefficient & $0.006(3)$ \\
& 4 & Largest diff. peak and hole & 0.311 and $-0.233 \mathrm{e}^{-3}$ \\
\hline
\end{tabular}





\section{SUPPLEMENT}

\subsection{Lifetime measurement of $\mathbf{1 7}$}

The measurements were carried out during a workshop of the company Horiba Scientific in Essen 2014 at an exhibited spectrometer of the DeltaFlex series. Unfortunately, the company only handed over a screenshot of the results without any raw data. This is why no clear graph could be prepared. In Figure 7.1, the abscissa represents the measured channels and the counts are given on the vertical axis. A value of 13.9 ns was received for the lifetime of $\mathbf{1 7}$.

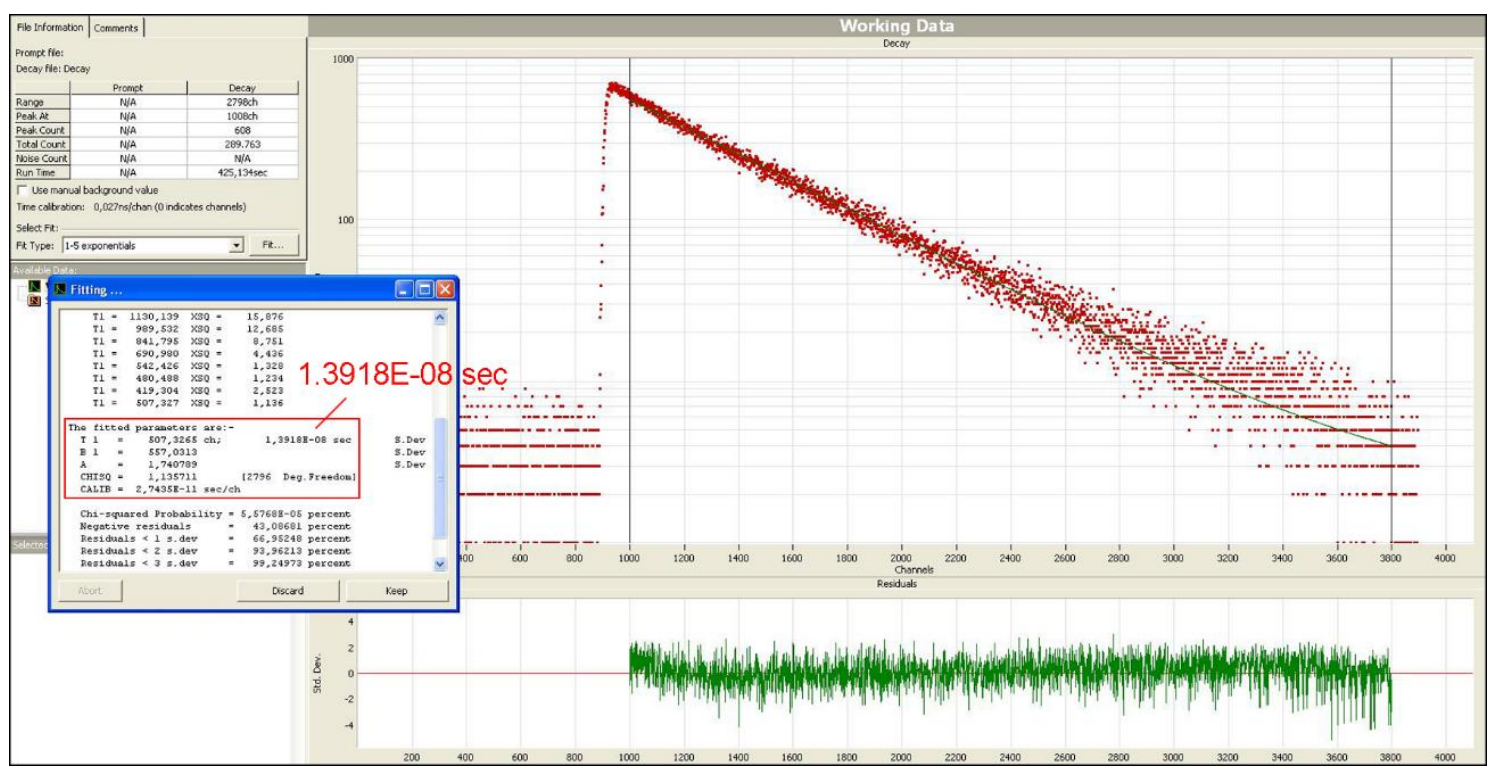

Figure 7.1: Lifetime measurement of 17 in methanol $\left(10^{-5} \mathrm{M}\right)$.

\subsection{NMR spectra}

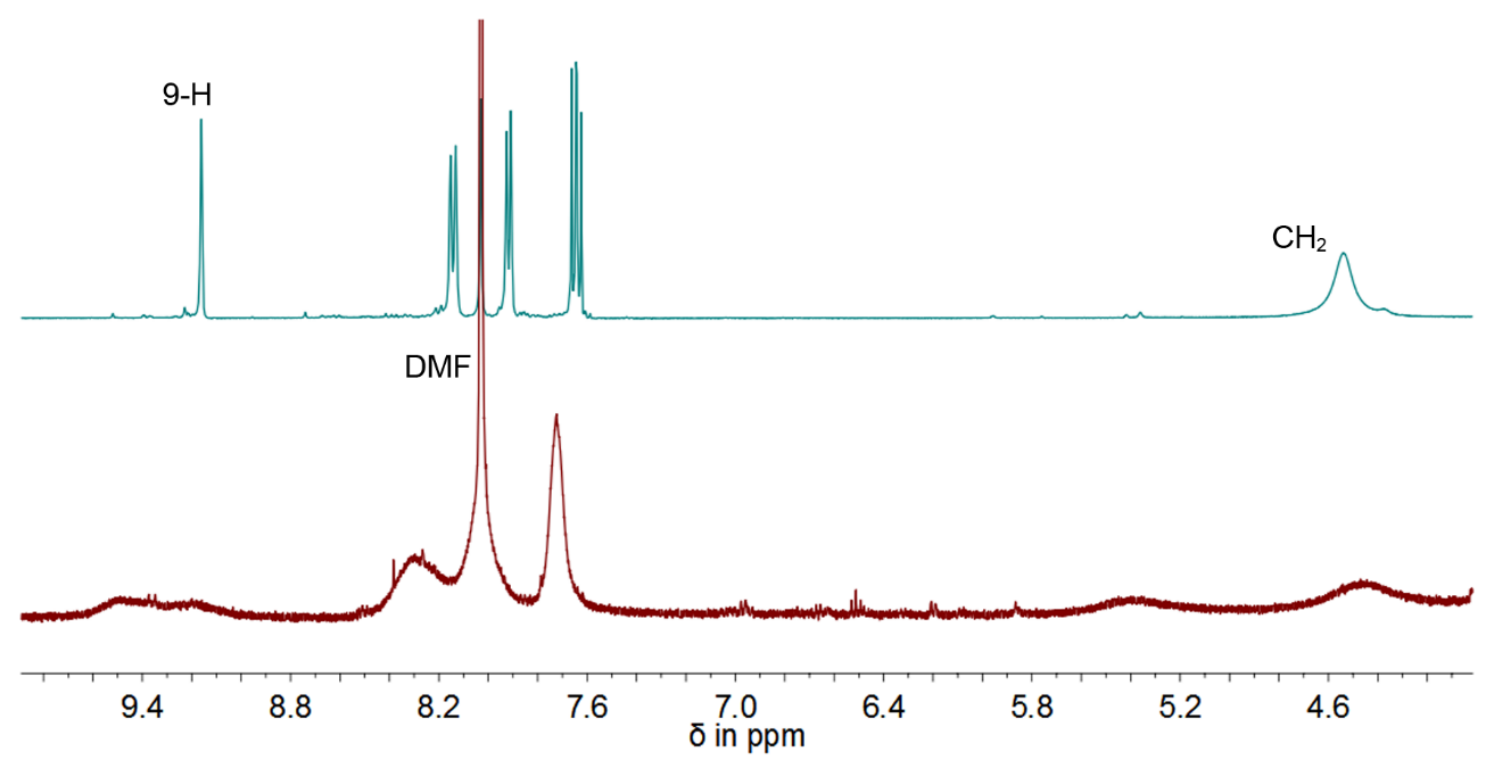

Figure 7.2: Stacked ${ }^{1} \mathrm{H}$ NMR spectra of 17 without (red) and with addition of 10 eq $\mathrm{NaBr}$ (turquois) in DMF- $d_{7}$ at ambient temperature. The signals are no longer broadened after the addition of $\mathrm{NaBr}$ which is why the dynamic processes can be attributed to the DMF/bromide exchange. 


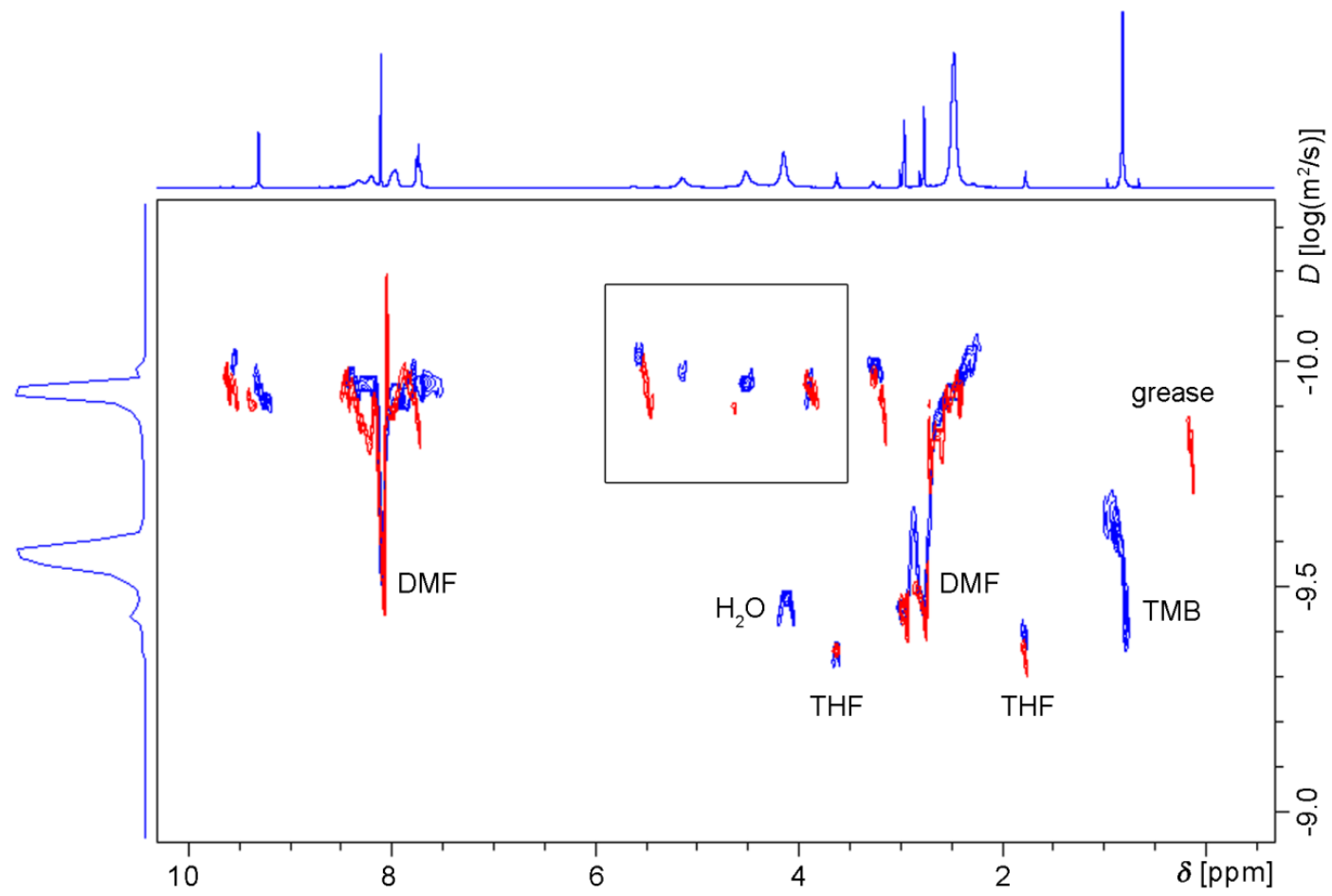

Figure 7.3: Overlay of ${ }^{1} \mathrm{H}$-DOSY NMR full spectra of 17 without (red) and with addition of $10 \mathrm{eq} \mathrm{NaBr}$ (blue) in DMF- $d_{7}$ at $243 \mathrm{~K}$.

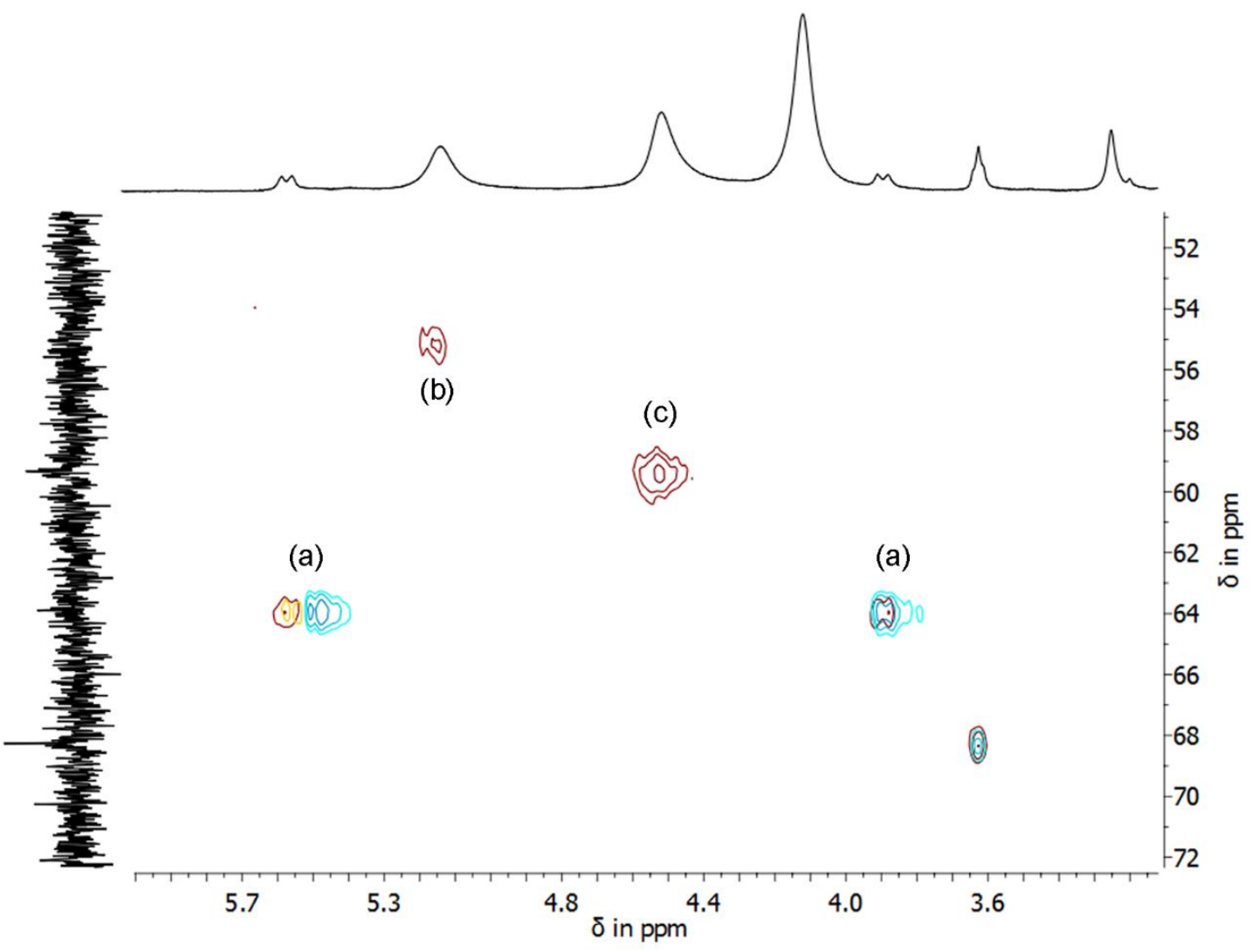

Figure 7.4: Superimposed ${ }^{1} \mathrm{H},{ }^{13} \mathrm{C}$-HSQC NMR spectra of $\mathbf{1 7}$ without (turquois) and with addition of $10 \mathrm{eq}$ $\mathrm{NaBr}$ (brown) in DMF- $d_{7}$ at $243 \mathrm{~K}$; a) $\left[(\mathrm{dmf})_{2} \mathrm{Zn}\left\{\left(\mathrm{Me}_{2} \mathrm{NCH}_{2}\right)_{2} \mathrm{Acr}\right\}\right]^{2+}$; b) $\left[(\mathrm{dmf}) \mathrm{ZnBr}\left\{\left(\mathrm{Me}_{2} \mathrm{NCH}_{2}\right)_{2} \mathrm{Acr}\right\}\right]^{+}$; c) $\left[\mathrm{ZnBr}_{2}\left\{\left(\mathrm{Me}_{2} \mathrm{NCH}_{2}\right)_{2} \mathrm{Acr}\right\}\right]$. This spectrum is in line with the theory of generating different species by adding $\mathrm{NaBr}$ to the NMR sample. The protons of (a), (b), and (c) couple to different carbon atoms and do not belong to one compound. 


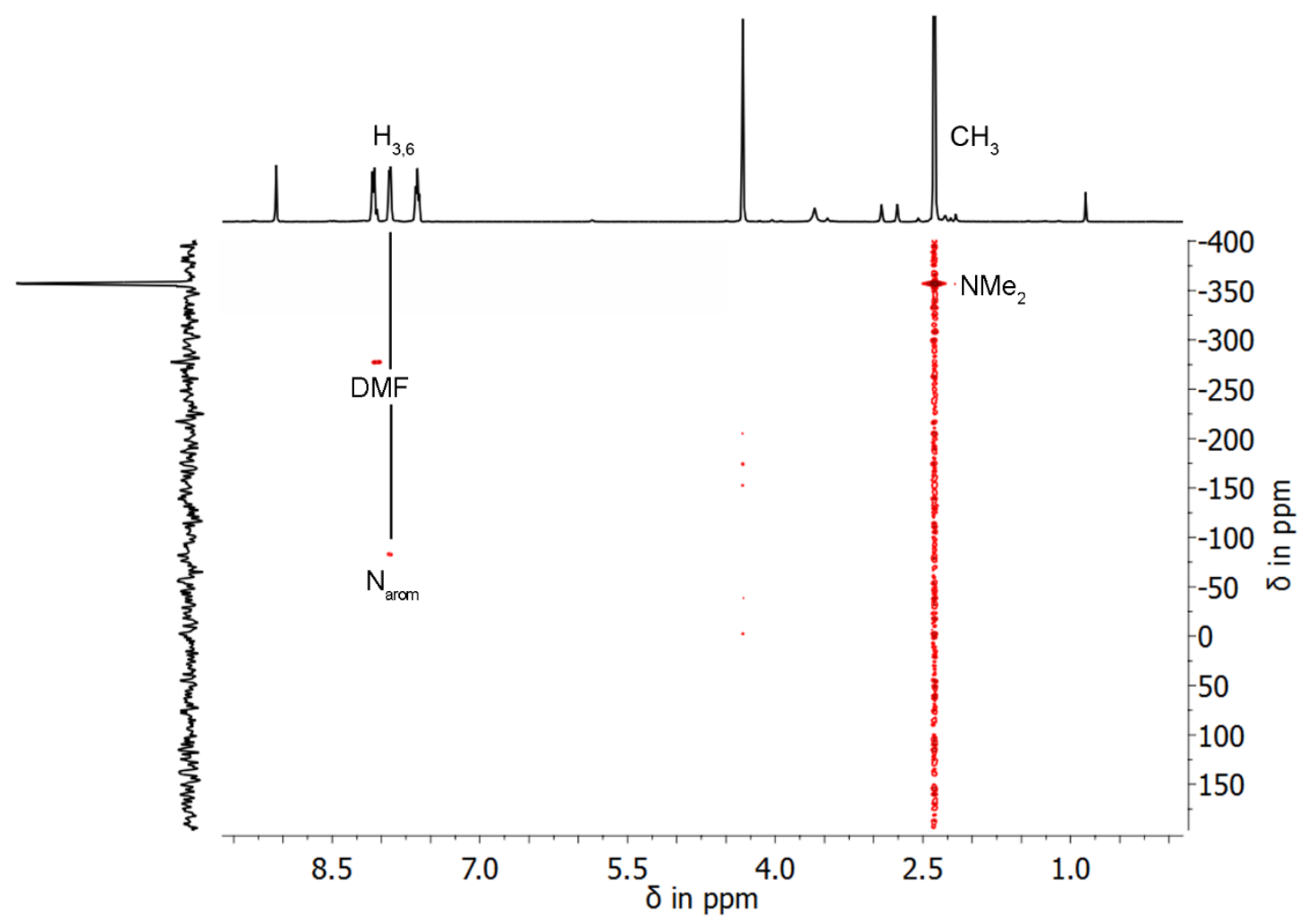

Figure 7.5: ${ }^{1} \mathrm{H},{ }^{15} \mathrm{~N}-\mathrm{HMBC}$ NMR spectrum of $\mathbf{1 5}$ in DMF- $d_{7}$ at $273 \mathrm{~K}$.

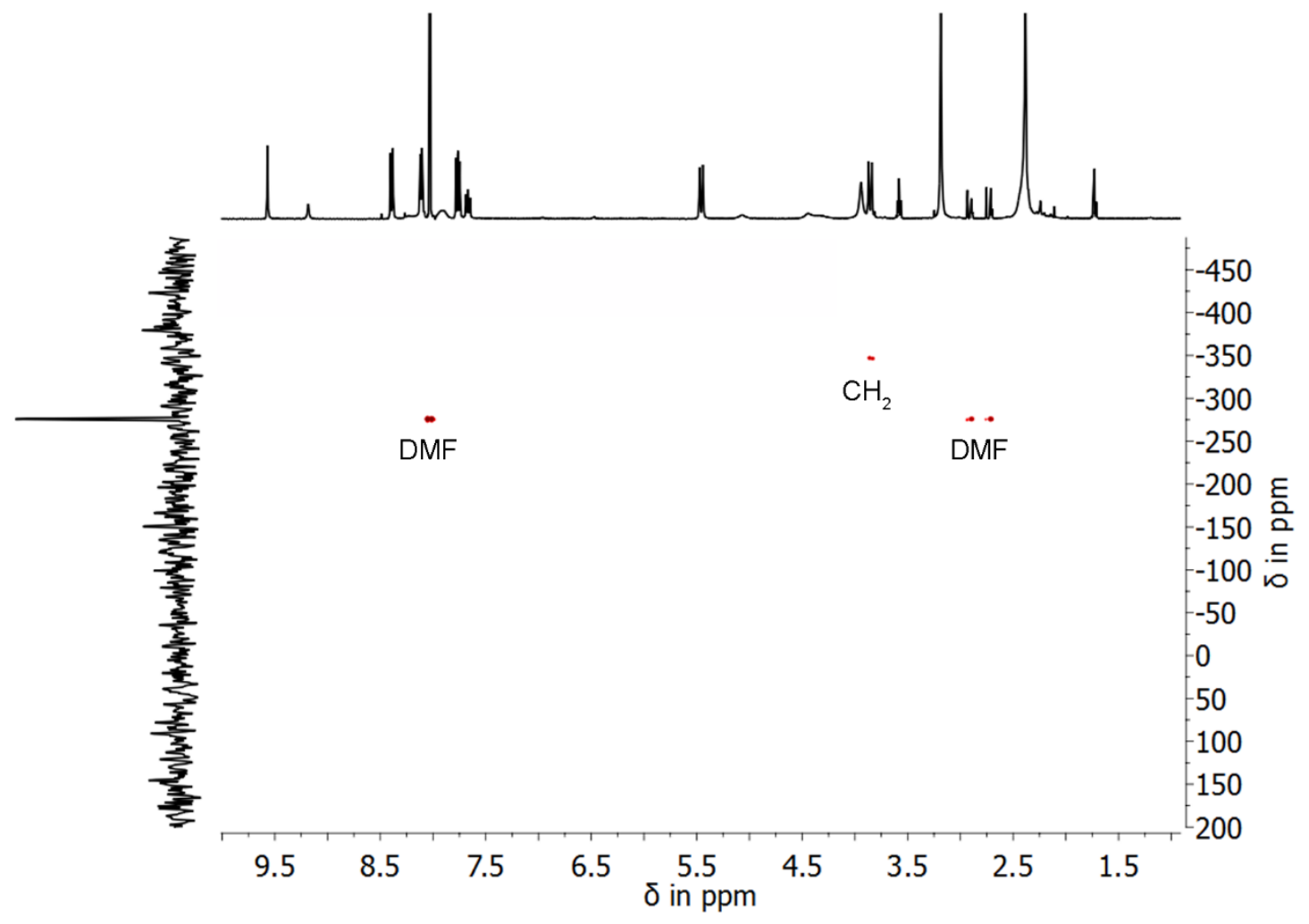

Figure 7.6: ${ }^{1} \mathrm{H},{ }^{15} \mathrm{~N}-\mathrm{HMBC}$ NMR spectrum of $\mathbf{1 7}$ in DMF- $d_{7}$ at $243 \mathrm{~K}$. 


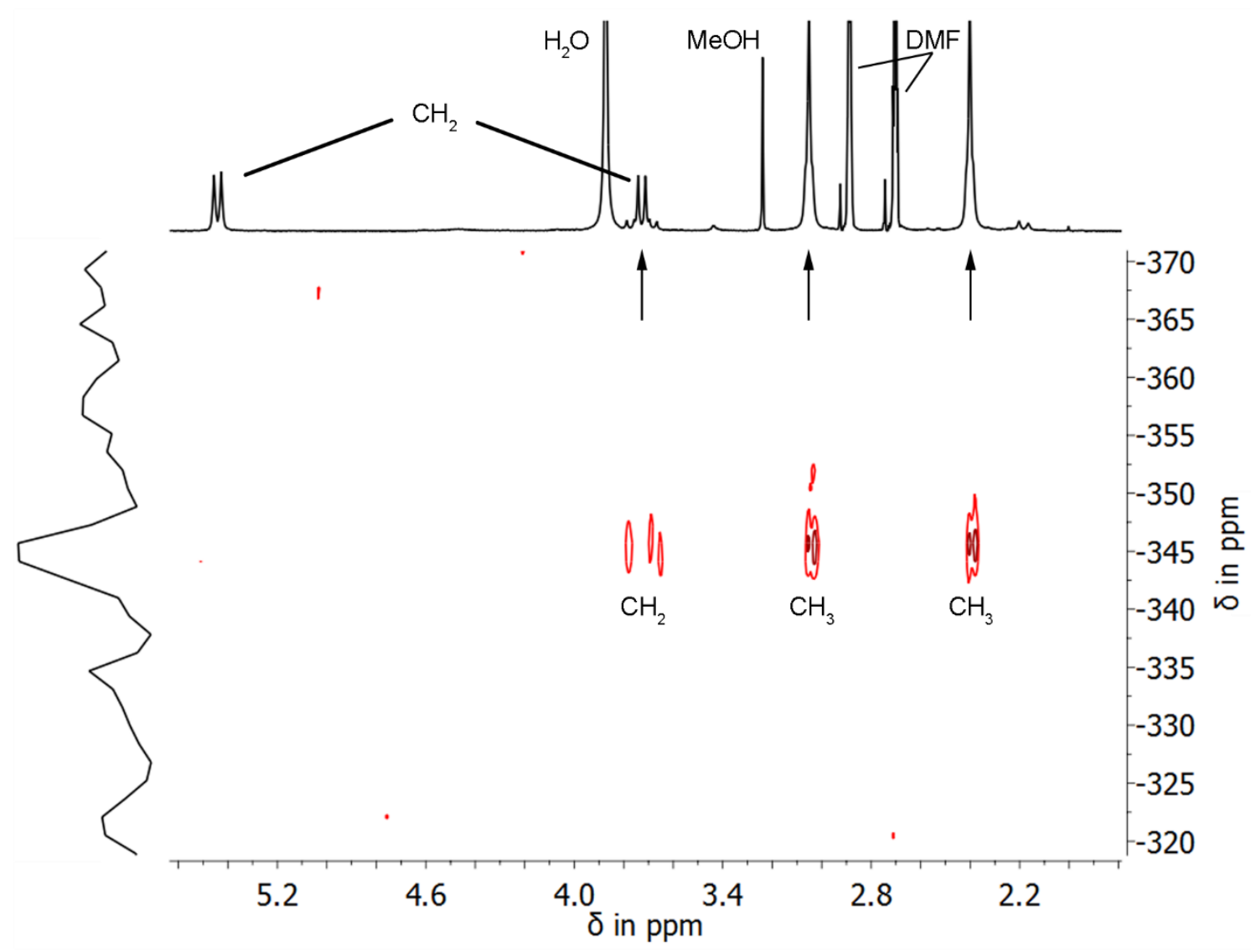

Figure 7.7: ${ }^{1} \mathrm{H},{ }^{113} \mathrm{Cd}-\mathrm{HMBC}$ NMR spectrum of 19 in DMF- $d_{7}$ at $243 \mathrm{~K}$. Only one proton of each $\mathrm{CH}_{2}$ group is coupled to the ${ }^{113} \mathrm{Cd}$ nucleus $(\delta=-345 \mathrm{ppm})$.

\subsection{Mass spectra}

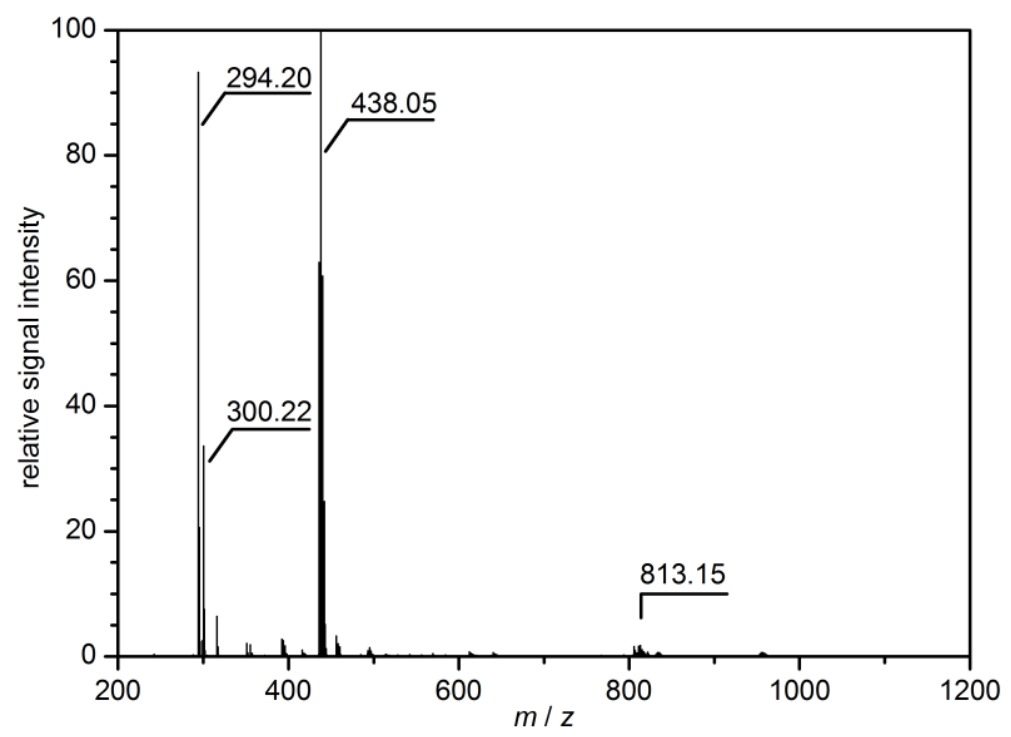

Figure 7.8: Positive-ion mode ESI-TOF mass spectrum of a solution of $\mathbf{1 7}$ in THF. 


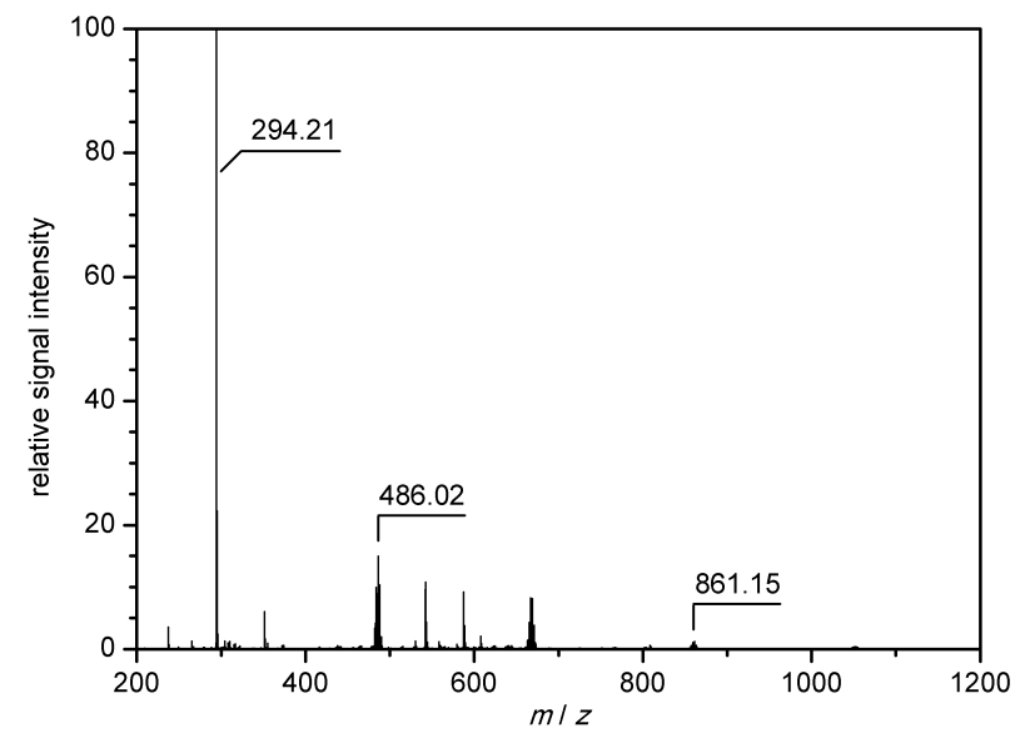

Figure 7.9: Positive-ion mode ESI-TOF mass spectrum of a solution of 19 in a $\mathrm{THF} / \mathrm{H}_{2} \mathrm{O}$ mixture.
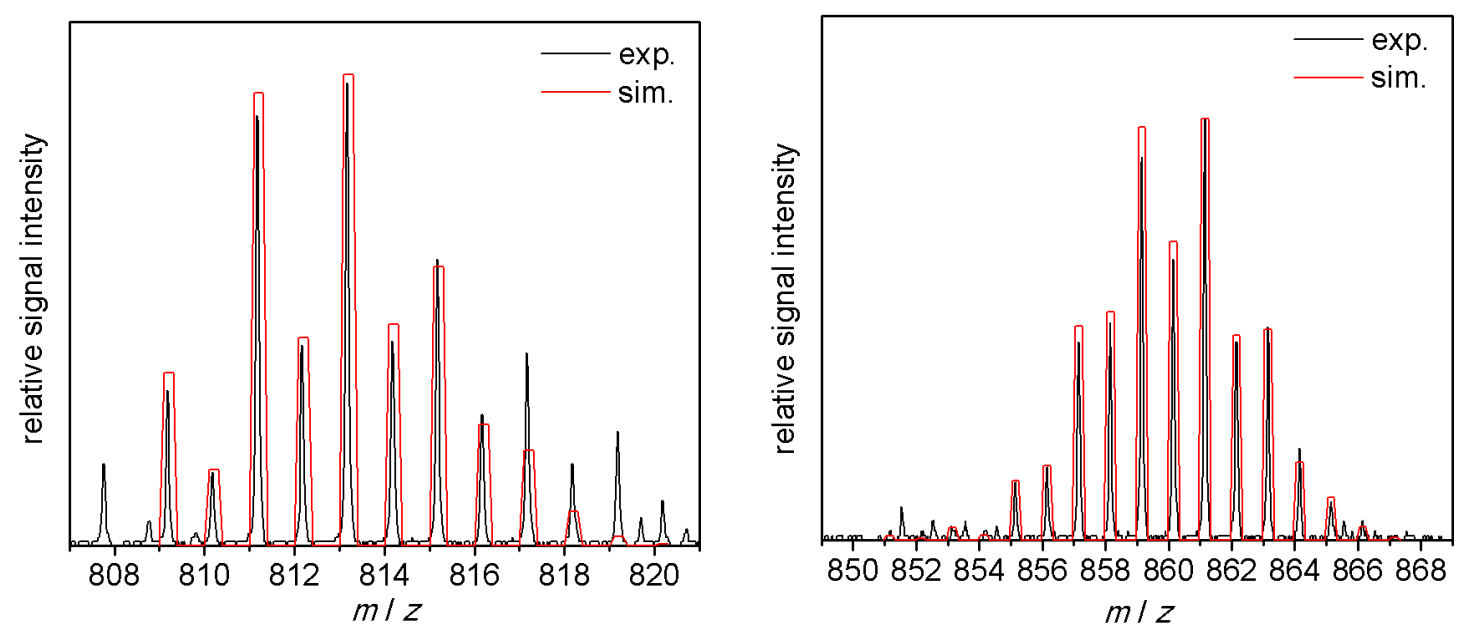

Figure 7.10: Parts of the mass spectra showing the simulated (red) and the experimental (black) isotope pattern of the cationic $\left[\left(\mathrm{C}_{19} \mathrm{H}_{23} \mathrm{~N}_{3}\right)_{2} \mathrm{HZnBr}_{2}\right]^{+}$(left) and $\left[\left(\mathrm{C}_{19} \mathrm{H}_{23} \mathrm{~N}_{3}\right)_{2} \mathrm{HCdBr}_{2}\right]^{+}$(right).
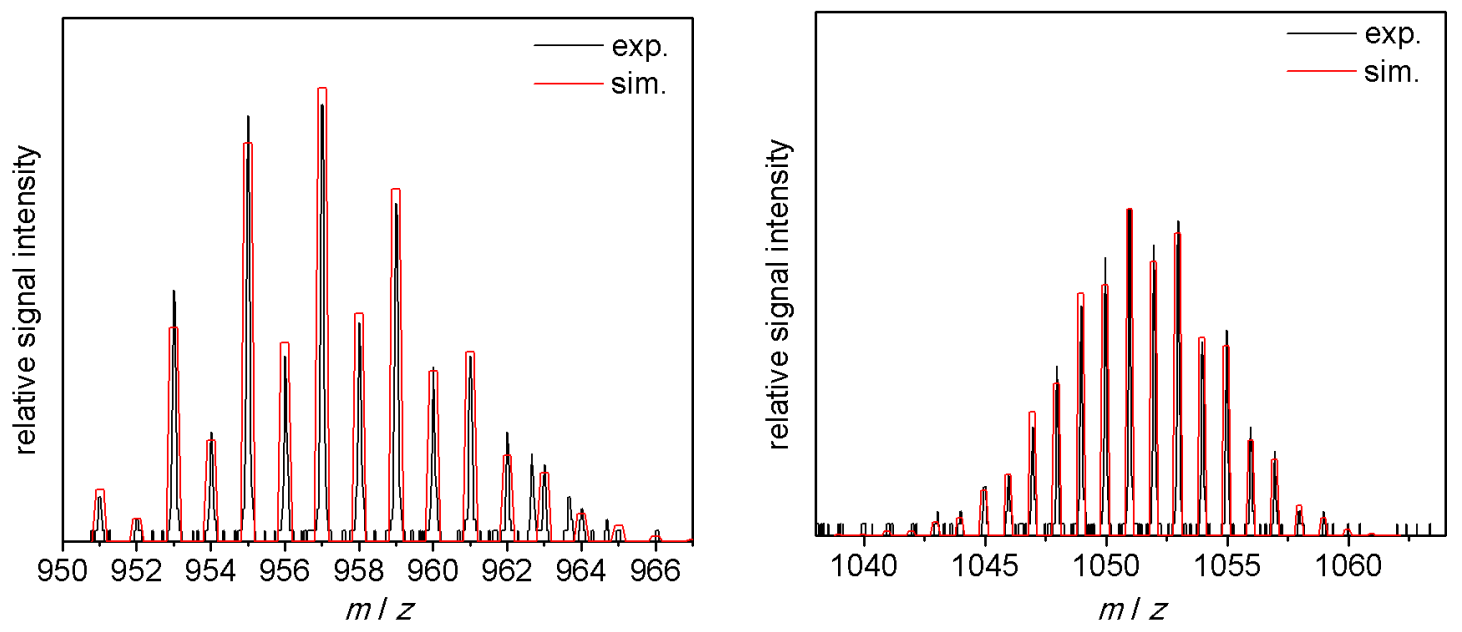

Figure 7.11: Parts of the mass spectra showing the simulated (red) and the experimental (black) isotope pattern of the cationic $\left[\left(\mathrm{C}_{19} \mathrm{H}_{23} \mathrm{~N}_{3}\right)_{2} \mathrm{Zn}_{2} \mathrm{Br}_{3}\right]^{+}$(left) and $\left[\left(\mathrm{C}_{19} \mathrm{H}_{23} \mathrm{~N}_{3}\right)_{2} \mathrm{Cd}_{2} \mathrm{Br}_{3}\right]^{+}$(right). 



\section{REFERENCES}

[1] B. Valeur, M. N. Berberan-Santos, J. Chem. Educ. 2011, 88, 731-738.

[2] A. U. Acuña, F. Amat-Guerri, in Fluorescence of Supermolecules, Polymers, and Nanosystems, Vol. 4 (Ed.: M. N. Berberan-Santos), Springer, Berlin, Heidelberg, 2008, pp. 3-20.

[3] A. U. Acuña, F. Amat-Guerri, P. Morcillo, M. Liras, B. Rodríguez, Org. Lett. 2009, 11, 3020-3023.

[4] (a) J. F. W. Herschel, Phil. Trans. R. Soc. London 1845, 135, 143-145; (b) D. Brewster, Phil. Mag. 1848, 32, 401-412.

[5] G. G. Stokes, Phil. Trans. R. Soc. London 1852, 142, 463-562.

[6] G. G. Stokes, Phil. Trans. R. Soc. London 1853, 143, 385-396.

[7] J. R. Lakowicz, Principles of Fluorescence Spectroscopy 3rd ed., Springer, New York, 2006, pp. 1-26.

[8] E. Becquerel, Ann. Chim. Phys. 1842, 9, 257-322.

[9] A. Jabłoński, Z. Physik 1935, 94, 38-46.

[10] M. Kasha, Discuss. Faraday Soc. 1950, 9, 14-19.

[11] N. J. Turro, V. Ramamurthy, J. C. Scaiano, Modern Molecular Photochemistry of Organic Molecules, 1st ed., University Science Books, Sausalito, 2010.

[12] (a) J. Franck, E. G. Dymond, Trans. Faraday Soc. 1926, 21, 536-542; (b) E. Condon, Phys. Rev. 1926, 28, 1182-1201.

[13] M. Sauer, J. Hofkens, J. Enderlein, Handbook of Fluorescence Spectroscopy and Imaging: From Single Molecules to Ensembles, Wiley-VCH, Weinheim, 2011, pp. 1-30.

[14] (a) A. P. de Silva, D. B. Fox, A. J. M. Huxley, N. D. McClenaghan, J. Roiron, Coord. Chem. Rev. 1999, 185-186, 297-306; (b) W.-Y. Wong, K. C.-F. Leung, J. F. Stoddart, Org. Biomol. Chem. 2010, 8, 23322343; (c) J. T. Hutt, J. Jo, A. Olasz, C.-H. Chen, D. Lee, Z. D. Aron, Org. Lett. 2012, 14, 3162-3165.

[15] A. P. de Silva, H. Q. N. Gunaratne, T. Gunnlaugsson, A. J. M. Huxley, C. P. McCoy, J. T. Rademacher, T. E. Rice, Chem. Rev. 1997, 97, 1515-1566.

[16] (a) R. A. Bissell, A. P. de Silva, H. Q. N. Gunaratne, P. L. M. Lynch, G. E. M. Maguire, K. R. A. S. Sandanayake, Chem. Soc. Rev. 1992, 21, 187-195; (b) J. F. Callan, A. P. de Silva, J. Ferguson, A. J. M. Huxley, A. M. O’Brien, Tetrahedron 2004, 60, 11125-11131; (c) A. Kumar, V. Kumar, K. K. Upadhyay, Analyst 2013, 138, 1891-1897.

[17] Y. Li, T. Liu, H. Liu, M.-Z. Tian, Y. Li, Acc. Chem. Res. 2014, 47, 1186-1198.

[18] T. Saha, A. Sengupta, P. Hazra, P. Talukdar, Photochem. Photobiol. Sci. 2014, 13, 1427-1433.

[19] J. B. Birks, Photophysics of Aromatic Molecules, 1st ed., Wiley - Interscience, London, New York, Sydney, Toronto, $\mathbf{1 9 7 0 .}$

[20] (a) R. A. Bissel, A. P. de Silva, H. Q. N. Gunaratne, P. L. M. Lynch, G. E. M. Maguire, C. P. McCoy, K. R. A. S. Sandanayake, in Photoinduced Electron Transfer V, Vol. 168 (Ed.: J. Mattay), Springer, Berlin, Heidelberg, 1993, pp. 229-230; (b) K. Kubo, in Topics in Fluorescence Spectroscopy, Vol. 9 (Eds.: C. D. Geddes, J. R. Lakowicz), Springer US, 2005, pp. 219-247; (c) A. P. de Silva, J. Phys. Chem. Lett. 2011, 2, 2865-2871.

[21] M. Asha Jhonsi, A. Kathiravan, J. Lumin. 2014, 145, 188-193.

[22] J. C. Beeson, M. E. Huston, D. A. Pollard, T. K. Venkatachalam, A. W. Czarnik, J. Fluoresc. 1993, 3, 65-68.

[23] R. S. Davidson, K. R. Trethewey, J. Chem. Soc., Chem. Commun. 1976, 827-829.

[24] (a) G. Greiner, I. Maier, J. Chem. Soc., Perkin Trans. 2 2002, 1005-1011; (b) A. P. de Silva, S. Uchiyama, in Luminescence Applied in Sensor Science, 1st ed. (Eds.: L. Prodi, M. Montalti, Z. Nelsi), Springer, Heidelberg, 2011.

[25] (a) Z. Fei, N. Kocher, C. J. Mohrschladt, H. Ihmels, D. Stalke, Angew. Chem. Int. Ed. 2003, 42, 783787; Angew. Chem. 2003, 115, 807-811; (b) G. Schwab, Dissertation, Göttingen, 2008; (c) D. Stern, Dissertation, Göttingen, 2009; (d) N. Finkelmeier, Dissertation, Göttingen, 2013; (e) S. Wandtke, Dissertation, Göttingen, 2014.

[26] N. Nijegorodov, R. Mabbs, D. P. Winkoun, Spectrochim. Acta, Part A 2003, 59, 595-606.

[27] (a) SciFinder, 2016; Chemical Abstracts Service: Columbus, OH, (accessed Feb 03, 2016); (b) The SciFinder search was refined by 'document types' with the filter categories 'book', 'journal', 'letter', 'report', and 'review'.

[28] A. Philips, Ber. Dtsch. Chem. Ges. 1895, 28, 1658-1660. 
[29] N. Beeharry, A. G. L. Di Rora, M. R. Smith, T. J. Yen, Cancer Biol. Ther. 2015, 16, 1397-1406.

[30] A. Opitz, E. Roemer, W. Haas, H. Görls, W. Werner, U. Gräfe, Tetrahedron 2000, 56, 5147-5155.

[31] (a) W. E. Rhine, J. Davis, G. Stucky, J. Am. Chem. Soc. 1975, 97, 2079-2085; (b) L.-J. Fan, Y. Zhang, W. E. Jones Jr., Macromolecules 2005, 38, 2844-2849.

[32] Y. Zou, D. D. Young, A. Cruz-Montanez, A. Deiters, Org. Lett. 2008, 10, 4661-4664.

[33] E. Campaigne, J. Ashby, J. Heterocycl. Chem. 1971, 8, 487-488.

[34] (a) J. v. Braun, J. Nelles, Ber. Dtsch. Chem. Ges. 1937, 70B, 1760-1766; (b) N. S. Prostakov, V. I. Kuznetsov, Chem. Heterocycl. Compd. 1980, 5, 525-529; (c) A.-S. Rebstock, F. Mongin, F. Trecourt, G. Queguiner, Org. Biomol. Chem. 2004, 2, 291-295.

[35] C. Graebe, H. Caro, J. Prakt. Chem. 1870, 2, 183-185.

[36] (a) A. Bernthsen, Ber. Dtsch. Chem. Ges. 1883, 16, 767-769; (b) A. Bernthsen, Liebigs Ann. Chem. 1884, 224, 1-56.

[37] (a) O. Tsuge, M. Nishinohara, M. Tashi, Bull. Chem. Soc. Jpn. 1963, 36, 1477-1485; (b) J. Joseph, E. Kuruvilla, A. T. Achuthan, D. Ramaiah, G. B. Schuster, Bioconjugate Chem. 2004, 15, 1230-1235; (c) J. Khalafy, R. H. Prager, N. M. Puspawati, Turk. J. Chem. 2008, 32, 755-763.

[38] (a) R. Fukuda, S. Takenaka, M. Takagi, J. Chem. Soc., Chem. Commun. 1990, 1028-1030; (b) E. Kikuta, M. Murata, N. Katsube, T. Koike, E. Kimura, J. Am. Chem. Soc. 1999, 121, 5426-5436; (c) K. E. Siters, M. A. Fountain, J. R. Morrow, Inorg. Chem. 2014, 53, 11540-11551.

[39] W. Kikuth, Dtsch. Med. Wochenschr. 1932, 58, 530-531.

[40] Z. Ma, J. R. Choudhury, M. W. Wright, C. S. Day, G. Saluta, G. L. Kucera, U. Bierbach, J. Med. Chem. 2008, 51, 7574-7580.

[41] J. Chiron, J.-P. Galy, Synlett 2003, 15, 2349-2350.

[42] V. Sourdon, S. Mazoyer, V. Pique, J.-P. Galy, Molecules 2001, 6, 673-682.

[43] (a) W. N. Lipscomb, N. Sträter, Chem. Rev. 1996, 96, 2375-2434; (b) R. McRae, P. Bagchi, S. Sumalekshmy, C. J. Fahrni, Chem. Rev. 2009, 109, 4780-4827.

[44] (a) J.-Y. Koh, S. W. Suh, B. J. Gwag, Y. Y. He, C. Y. Hsu, D. W. Choi, Science 1996, 272, 1013-1016; (b) A. I. Bush, Curr. Opin. Chem. Biol. 2000, 4, 184-191.

[45] (a) M. Cortesi, E. Fridman, A. Volkov, S. S. Shilstein, R. Chechik, A. Breskin, D. Vartsky, N. Kleinman, G. Kogan, E. Moriel, V. Gladysh, M. Huszar, J. Ramon, G. Raviv, The Prostate 2008, 68, 994-1006; (b) Z. Medarova, S. K. Ghosh, M. Vangel, R. Drake, A. Moore, Am. J. Cancer Res. 2014, 4, 385-393.

[46] (a) J. M. Berg, Y. Shi, Science 1996, 271, 1081-1085; (b) N. Wellinghausen, M. Martin, L. Rink, Eur. J. Immunol. 1997, 27, 2529-2535; (c) M. J. Salgueiro, M. Zubillaga, A. Lysionek, M. I. Sarabia, R. Caro, T. De Paoli, A. Hager, R. Weill, J. Boccio, Nutr. Res. 2000, 20, 737-755.

[47] (a) K. Nogawa, A. Ishizaki, E. Kobayashi, Environ. Res. 1979, 18, 397-409; (b) C. Tohyama, Z. A. Shaikh, K. Nogawa, E. Kobayashi, R. Honda, Arch. Toxicol. 1982, 50, 159-166.

[48] (a) A. Ajayaghosh, P. Carol, S. Sreejith, J. Am. Chem. Soc. 2005, 127, 14962-14963; (b) K. Ono, J. K. Klosterman, M. Yoshizawa, K. Sekiguchi, T. Tahara, M. Fujita, J. Am. Chem. Soc. 2009, 131, 1252612527; (c) Z. Xu, J. Yoon, D. R. Spring, Chem. Soc. Rev. 2010, 39, 1996-2006; (d) H. Woo, S. Cho, Y. Han, W.-S. Chae, D.-R. Ahn, Y. You, W. Nam, J. Am. Chem. Soc. 2013, 135, 4771-4787; (e) M. Yamashina, M. M. Sartin, Y. Sei, M. Akita, S. Takeuchi, T. Tahara, M. Yoshizawa, J. Am. Chem. Soc. 2015, 137, 9266-9269.

[49] M. S. Park, K. M. K. Swamy, Y. J. Lee, H. N. Lee, Y. J. Jang, Y. H. Moon, J. Yoon, Tetrahedron Lett. 2006, 47, 8129-8132.

[50] H. N. Lee, K. M. K. Swamy, S. K. Kim, J.-Y. Kwon, Y. Kim, S.-J. Kim, Y. J. Yoon, J. Yoon, Org. Lett. 2007, 9, 243-246.

[51] W. Scenger, Principles of Nucleic Acid Structure, Springer, New York, 1998.

[52] S. Szarvas, Z. Majer, P. Huszthy, B. Vermes, M. Hollósi, Enantiomer 2002, 7, 241-249.

[53] J. Kertész, I. Móczár, A. Kormos, P. Baranyai, M. Kubinyi, K. Tóth, P. Huszthy, Tetrahedron: Asymmetry 2011, 22, 684-689.

[54] T. Németh, S. Lévai, A. Kormos, J. Kupai, T. Tóth, G. T. Balogh, P. Huszthy, Chirality 2014, 26, 651654.

[55] I. Móczár, P. Huszthy, A. Mezei, M. Kádár, J. Nyitrai, K. Tóth, Tetrahedron 2010, 66, 350-358.

[56] J. Kertész, B. Bognár, A. Kormos, I. Móczár, P. Baranyai, M. Kubinyi, T. Kálai, K. Hideg, P. Huszthy, Tetrahedron 2011, 67, 8860-8864. 
[57] Y. Wang, X. Hu, L. Wang, Z. Shang, J. Chao, W. Jin, Sens. Actuators, B 2011, 156, 126-131.

[58] E. V. Solovyeva, L. A. Myund, G. L. Starova, E. M. Dem'yanchuk, A. A. Makarov, A. S. Denisova, J. Mol. Struct. 2014, 1063, 235-241.

[59] E. V. Solovyeva, G. L. Starova, L. A. Myund, A. S. Denisova, Polyhedron 2016, 106, 1-9.

[60] R. Ghosh, A. Nandi, D. K. Palit, Phys. Chem. Chem. Phys. 2016, 18, 7661-7671.

[61] Y. Jiao, B. Zhu, J. Chen, X. Duan, Theranostics 2015, 5, 173-187.

[62] N. Dash, A. Malakar, M. Kumar, B. B. Mandal, G. Krishnamoorthy, Sens. Actuators, B 2014, 202 , 1154-1163.

[63] A. Visscher, Master thesis, Göttingen, 2011.

[64] A. P. Krapcho, T. P. Gilmor, J. Heterocycl. Chem. 1998, 35, 669-674.

[65] A. A. Leon, G. Daub, I. R. Silverman, J. Org. Chem. 1984, 49, 4544-4545.

[66] (a) S. M. Duan, J. Turk, J. Speigle, J. Corbin, J. Masnovi, R. J. Baker, J. Org. Chem. 2000, 65, 30053009; (b) R. Brückner, Reaktionsmechanismen, Vol. 3, Elsevier, München, 2004.

[67] R. M. Acheson, The Chemistry of Heterocyclic Compounds, Acridines, 2nd ed., John Wiley \& Sons, Toronto, 2009.

[68] J.-P. Galy, J. Chiron, Synthesis 2004, 3, 313-325.

[69] K. Fukui, T. Yonezawa, C. Nagata, H. Shingu, J. Chem. Phys. 1954, 22, 1433-1442.

[70] (a) U. Groth, L. Richter, U. Schöllkopf, Liebigs Ann. Chem. 1992, 1992, 199-202; (b) J. K. Ray, B. C. Roy, G. K. Kar, J. Org. Chem. 1996, 61, 1863-1866.

[71] J. J. Gassensmith, E. Arunkumar, L. Barr, J. M. Baumes, K. M. DiVittorio, J. R. Johnson, B. C. Noll, B. D. Smith, J. Am. Chem. Soc. 2007, 129, 15054-15059.

[72] G. A. Olah, R. Malhotra, S. C. Narang, Nitration: Methods and Mechanisms, Wiley-VCH, New York, 1989.

[73] C. Graebe, H. Caro, Liebigs Ann. Chem. 1871, 158, 265-281.

[74] R. M. Acheson, The Chemistry of Heterocyclic Compounds, Acridines, 2nd ed., John Wiley \& Sons, Toronto, 2009, pp. 1-8.

[75] R. M. Acheson, B. Adcock, G. M. Glover, L. E. Sutton, J. Chem. Soc. 1960, 3367-3371.

[76] K. Lehmstedt, Ber. Dtsch. Chem. Ges. 1938, 71, 808-814.

[77] P. Strazzolini, A. G. Giumanini, A. Runcio, Tetrahedron Lett. 2001, 42, 1387-1389.

[78] M. J. Plater, P. Barnes, L. K. McDonald, S. Wallace, N. Archer, T. Gelbrich, P. N. Horton, M. B. Hursthouse, Org. Biomol. Chem. 2009, 7, 1633-1641.

[79] S. M. S. Chauhan, R. Singh, Geetanjali, Synth. Commun. 2003, 33, 2899-2906.

[80] (a) H. Rao, Y. Jin, H. Fu, Y. Jiang, Y. Zhao, Chem.-Eur. J. 2006, 12, 3636-3646; (b) X. Guo, H. Rao, H. Fu, Y. Jiang, Y. Zhao, Adv. Synth. Catal. 2006, 348, 2197-2202.

[81] (a) K. Shimi, G. Boyer, J.-P. Finet, J.-P. Galy, Lett. Org. Chem. 2004, 1, 34-36; (b) M. Binaschi, A. Boldetti, M. Gianni, C. A. Maggi, M. Gensini, M. Bigioni, M. Parlani, A. Giolitti, M. Fratelli, C. Valli, M. Terao, E. Garattini, ACS Med. Chem. Lett. 2010, 1, 411-415.

[82] Y. Kitahara, T. Mizuno, A. Kubo, Tetrahedron 2004, 60, 4283-4288.

[83] L. Krause, R. Herbst-Irmer, G. M. Sheldrick, D. Stalke, J. Appl. Cryst. 2015, 48, 3-10.

[84] P. Müller, R. Herbst-Irmer, A. L. Spek, T. R. Schneider, M. R. Sawaya, in Crytal Structure Refinement - A Crystallographer's Guide to SHELXL, 1st ed. (Ed.: P. Müller), Oxford University Press, New York, 2006.

[85] (a) H. Langhals, T. Potrawa, H. Nöth, G. Linti, Angew. Chem. Int. Ed. 1989, 28, 478-480; Angew. Chem. 1998, 101, 497-499; (b) X.-H. Bu, M.-L. Tong, J.-R. Li, H.-C. Chang, L.-J. Li, S. Kitagawa, CrystEngComm 2005, 7, 411-416; (c) B. Dong, M. Wang, C. Xu, Q. Feng, Y. Wang, Cryst. Growth Des. 2012, 12, 5986-5993; (d) M. Yamamura, S. Ikuma, T. Nabeshima, J. Mol. Struct. 2015, 1093, 5964.

[86] (a) A. Mukherjee, S. Tothadi, G. R. Desiraju, Acc. Chem. Res. 2014, 47, 2514-2524; (b) G. R. Desiraju, R. Parthasarathy, J. Am. Chem. Soc. 1989, 111, 8725-8726.

[87] A. Bondi, J. Phys. Chem. 1964, 68, 441-451.

[88] (a) A. Achari, S. Neidle, Acta Cryst. B 1977, 33, 3269-3270; (b) A. J. Adamson, Y. Archambeau, R. E. Banks, B. Beagley, Acta Cryst. C 1994, 50, 967-971.

[89] T. Steiner, Angew. Chem. Int. Ed. 2002, 41, 48-76; Angew. Chem. 2002, 114, 50-80. 
[90] F. Karagöz, O. Güney, M. Kandaz, A. T. Bilgiçli, J. Lumin. 2012, 132, 2736-2740.

[91] W. B. Jensen, The Lewis Acid-Base Concepts. An Overview, John Wiley \& Sons, New York, 1980.

[92] (a) Y. Umezawa, S. Tsuboyama, H. Takahashi, J. Uzawa, M. Nishio, Tetrahedron 1999, 55, $10047-$ 10056; (b) M. Nishio, Y. Umezawa, K. Honda, S. Tsuboyama, H. Suezawa, CrystEngComm 2009, 11, 1757-1788.

[93] M. Kasha, Radiat. Res. 1960, 2, 243-275.

[94] J. B. Birks, L. G. Christophorou, Spectrochim. Acta 1963, 19, 401-410.

[95] B. Cadioli, E. Gallinella, C. Coulombeau, H. Jobic, G. Berthier, J. Phys. Chem. 1993, 97, 7844-7856.

[96] M. D. Hanwell, D. E. Curtis, D. C. Lonie, T. Vandermeersch, E. Zurek, G. R. Hutchison, J. Cheminform. 2012, 4, 1-17.

[97] M. E. Huston, K. W. Haider, A. W. Czarnik, J. Am. Chem. Soc. 1988, 110, 4460-4462.

[98] (a) J. Hicks, E. J. Underhill, C. E. Kefalidis, L. Maron, C. Jones, Angew. Chem. Int. Ed. 2015, 54, 10000-10004; Angew. Chem. 2015, 127, 10138-10142; (b) B. Roy, A. K. Ghosh, S. Srivastava, P. D'Silva, P. S. Mukherjee, J. Am. Chem. Soc. 2015, 137, 11916-11919.

[99] M. Laronze-Cochard, Y.-M. Kim, B. Brassart, J.-F. Riou, J.-Y. Laronze, J. Sapi, Eur. J. Med. Chem. 2009, 44, 3880-3888.

[100] J. F. Mammone, S. K. Sharma, M. Nicol, J. Phys. Chem. 1980, 84, 3130-3134.

[101] R. K. Haynes, S. C. Vonwiller, M. R. Luderer, in e-EROS Encyclopedia of Reagents for Organic Synthesis, John Wiley \& Sons, 2006.

[102] U. Knips, F. Huber, Z. Naturforsch. 1983, 38b, 434-436.

[103] (a) A. Gafni, L. Brand, Chem. Phys. Lett. 1978, 58, 346-350; (b) J. R. Lakowicz, A. Balter, Biophys. Chem. 1982, 16, 117-132.

[104] D. D. Pant, G. C. Joshi, H. B. Tripathi, J. Phys. 1986, 27, 161-170.

[105] (a) P. Gangola, N. B. Joshi, D. D. Pant, Chem. Phys. Lett. 1979, 60, 329-331; (b) M. K. Sarangi, D. Dey, S. Basu, J. Phys. Chem. A 2011, 115, 128-135.

[106] (a) M. Kasha, J. Chem. Phys. 1952, 20, 71-74; (b) A. Martin, R. Narayanaswamy, Sens. Actuators, B 1997, 39, 330-333.

[107] J. R. Lakowicz, Principles of Fluorescence Spectroscopy 3rd ed., Springer, New York, 2006, pp. 205235.

[108] M. Hofmann, H. Graener, Chem. Phys. 1996, 206, 129-137.

[109] F. Neese, WIREs Comput. Mol. Sci. 2012, 2, 73-78.

[110] V. P. Zvolinskii, N. I. Nizhegorodov, V. V. Nikiforov, V. V. Dorogov, A. N. Gusarov, Sov. Phys. J. 1977, 20, 701-706.

[111] A. Visscher, S. Bachmann, C. Schnegelsberg, T. Teuteberg, R. A. Mata, D. Stalke, Dalton Trans. 2016, 45, 5689-5699.

[112] I. Negrón-Encarnación, R. Arce, M. Jiménez, J. Phys. Chem. A 2005, 109, 787-797.

[113] J. N. Murrell, Proc. Phys. Soc. A 1955, 68, 969-975.

[114] P. Nikolov, H. Görner, J. Photochem. Photobiol., A 1996, 101, 137-144.

[115] B. Ma, F. Zeng, X. Li, S. Wu, Chem. Commun. 2012, 48, 6007-6009.

[116] (a) S. Karuppannan, J.-C. Chambron, Chem. Asian J. 2011, 6, 964-984; (b) M. Shellaiah, Y.-H. Wu, A. Singh, M. V. Ramakrishnam Raju, H.-C. Lin, J. Mat. Chem. A 2013, 1, 1310-1318.

[117] H. Friebolin, Basic One- and Two-Dimensional NMR Spectroscopy, 5th ed., Wiley-VCH Verlag, Weinheim, 2011.

[118] J. Keeler, Understanding NMR Spectroscopy, 2nd ed., John Wiley \& Sons, 2010, pp. 300-303526.

[119] T. Steiner, Acta Cryst. B 1998, 54, 456-463.

[120] G. A. Jeffrey, An Introduction to Hydrogen Bonding, Oxford University Press, Oxford, 1997.

[121] Y. Wang, L. Shi, H. S. Sun, Z. Shang, J. Chao, W. Jin, J. Lumin. 2013, 139, 16-21.

[122] HORIBA, FluoroMax-4 Spectrofluorometer Operation Manual 2006, 2.2-2.6.

[123] G. A. Carlson, J. P. McReynolds, F. H. Verhoek, J. Am. Chem. Soc. 1945, 67, 1334-1339.

[124] R. D. Shannon, Acta Cryst. A 1976, 32, 751-767.

[125] H. Irving, R. J. P. Williams, J. Chem. Soc. 1953, 3192-3210. 
[126] (a) V. Balzani, Tetrahedron 1992, 48, 10443-10514; (b) L. Fabbrizzi, M. Licchelli, P. Pallavicini, A. Perotti, A. Taglietti, D. Sacchi, Chem.-Eur. J. 1996, 2, 75-82; (c) V. V. Volchkov, V. L. Ivanov, B. M. Uzhinov, J. Fluoresc. 2009, 20, 299-303.

[127] (a) J. D. Hoeschele, J. E. Turner, M. W. England, Sci. Total Environ. 1991, 109/1 10, 477-492; (b) R. M. Izatt, K. Pawlak, J. S. Bradshaw, R. L. Bruening, Chem. Rev. 1991, 91, 1721-2085.

[128] (a) C. Gunanathan, L. J. W. Shimon, D. Milstein, J. Am. Chem. Soc. 2009, 131, 3146-3147; (b) X. Ye, P. N. Plessow, M. K. Brinks, M. Schelwies, T. Schaub, F. Rominger, R. Paciello, M. Limbach, P. Hofmann, J. Am. Chem. Soc. 2014, 136, 5923-5929.

[129] Cambridge Structural Database, v5.37 (November 2015), Cambridge, UK, 2015.

[130] U. Flierler, D. Stalke, in Structure and Bonding, Vol. 146 (Ed.: D. Stalke), Springer, Berlin, New York, 2012, pp. 1-20.

[131] (a) A. D. Becke, Phys. Rev. A 1988, 38, 3098-3100; (b) C. Lee, W. Yang, R. G. Parr, Phys. Rev. B 1988, 37, 785-789; (c) A. D. Becke, J. Chem. Phys. 1993, 98, 5648-5652; (d) P. J. Stephens, F. J. Devlin, C. F. Chabalowski, M. J. Frisch, J. Phys. Chem. 1994, 98, 11623-11627; (e) F. Weigend, R. Ahlrichs, Phys. Chem. Chem. Phys. 2005, 7, 3297-3305; (f) S. Grimme, J. Antony, S. Ehrlich, H. Krieg, J. Chem. Phys. 2010, 132, 154104-154119.

[132] (a) J. P. Foster, F. Weinhold, J. Am. Chem. Soc. 1980, 102, 7211-7218; (b) A. E. Reed, R. B. Weinstock, F. Weinhold, J. Chem. Phys. 1985, 83, 735-746; (c) E. D. Glendening, J. K. Badenhoop, A. E. Reed, J. E. Carpenter, J. A. Bohmann, C. M. Morales, F. Weinhold, GenNBO 5.9, University of Wisconsin, Madison, 2009.

[133] A. E. Reed, L. A. Curtiss, F. Weinhold, Chem. Rev. 1988, 88, 899-926.

[134] C. Maaß, D. M. Andrada, R. A. Mata, R. Herbst-Irmer, D. Stalke, Inorg. Chem. 2013, 52, 9539-9548.

[135] A. Klamt, G. Schüürmann, J. Chem. Soc., Perkin Trans. 2 1993, 799-805.

[136] R. Neufeld, D. Stalke, Chem. Sci. 2015, 6, 3354-3364.

[137] (a) H. Gornitzka, D. Stalke, Eur. J. Inorg. Chem. 1998, 311-317; (b) T. E. Wood, B. Berno, C. S. Beshara, A. Thompson, J. Org. Chem. 2006, 71, 2964-2971.

[138] M. Karplus, J. Am. Chem. Soc. 1963, 85, 2870-2871.

[139] X. Lang, L. Li, Y. Chen, Q. Sun, Q. Wu, F. Liu, C. Tan, H. Liu, C. Gao, Y. Jiang, Bioorg. Med. Chem. 2013, 21, 4170-4177.

[140] (a) P. Job, Ann. Chim. Appl. 1928, 9, 113-203; (b) J. S. Renny, L. L. Tomasevich, E. H. Tallmadge, D. B. Collum, Angew. Chem. Int. Ed. 2013, 52, 11998-12013; Angew. Chem. 2013, 125, 12218-12234.

[141] C. Steinmetzger, Master thesis, Göttingen, 2015.

[142] P. Molz, H. J. Skrzipczyk, H. Luebbers, H. Strecker, G. Schnorr, T. Kinkel, Ger. Offen. DE 3628573, 1988; Chem. Abstr. 1988, 109: 89359.

[143] O. S. Radchenko, E. N. Sigida, N. N. Balaneva, P. S. Dmitrenok, V. L. Novikov, J. Heterocycl. Chem. 2011, 48, 209-214.

[144] L. Liu, Master thesis, Göttingen, 2015.

[145] C.-J. J. Wu, C. Xue, Y.-M. Kuo, F.-T. Luo, Tetrahedron 2005, 61, 4735-4741.

[146] (a) W. Schlenk, A. Thal, Ber. Dtsch. Chem. Ges. 1913, 46, 2840-2854; (b) T. T. Tidwell, Angew. Chem. Int. Ed. 2001, 40, 331-337; Angew. Chem. 2001, 113, 343-349; (c) Georg-August-University Göttingen, Virtuelles Labor, www.stalke.chemie.uni-goettingen.de/virtuelles_labor/en.html; access at 23/03/2016.

[147] A. Schäfer, H. Horn, R. Ahlrichs, J. Chem. Phys. 1992, 97, 2571-2577.

[148] S. Grimme, S. Ehrlich, L. Goerigk, J. Comput. Chem. 2011, 32, 1456-1465.

[149] (a) A. D. Becke, E. R. Johnson, J. Chem. Phys. 2005, 123, 154101; (b) E. R. Johnson, A. D. Becke, J. Chem. Phys. 2005, 123, 024101; (c) E. R. Johnson, A. D. Becke, J. Chem. Phys. 2006, 124, 174104.

[150] E. F. Valeev, LIBINT: A library for the evaluation of molecular integrals of many-body operators over Gaussian functions, Version 2.1.0 (beta), 2014; http://libint.valeyev.net/.

[151] K. Eichkorn, F. Weigend, O. Treutler, R. Ahlrichs, Theor. Chem. Acc. 1997, 97, 119-124.

[152] D. Andrae, U. Häußermann, M. Dolg, H. Stoll, H. Preuß, Theor. Chim. Acta 1990, 77, 123-141.

[153] (a) E. J. Baerends, D. E. Ellis, P. Ros, Chem. Phys. 1973, 2, 41-51; (b) J. L. Whitten, J. Chem. Phys. 1973, 58, 4496-4501; (c) F. Neese, F. Wennmohs, A. Hansen, U. Becker, Chem. Phys. 2009, 356, 98109.

[154] J. R. Lakowicz, Principles of Fluorescence Spectroscopy 3rd ed., Springer, New York, 2006, pp. $27-61$.

[155] HORIBA, FluorEssence v3.0, Edison, 2008. 
[156] H. J. Svec, Int. J. Mass Spectrom. Ion Processes 1985, 66, 3-29.

[157] J. F. d. 1. Mora, G. J. Van Berkel, C. G. Enke, R. B. Cole, M. Martinez-Sanchez, J. B. Fenn, J. Mass Spectrom. 2000, 35, 939-952.

[158] H. E. Gottlieb, V. Kotlyar, A. Nudelman, J. Org. Chem. 1997, 62, 7512-7515.

[159] (a) T. Kottke, D. Stalke, J. Appl. Cryst. 1993, 26, 615-619; (b) T. Kottke, R. J. Lagow, D. Stalke, J. Appl. Cryst. 1996, 29, 465-468; (c) D. Stalke, Chem. Soc. Rev. 1998, 27, 171-178.

[160] T. Schulz, K. Meindl, D. Leusser, D. Stern, J. Graf, C. Michaelsen, M. Ruf, G. M. Sheldrick, D. Stalke, J. Appl. Cryst. 2009, 42, 885-891.

[161] Bruker AXS Inc., SAINT v8.30C, Bruker Apex CCD, SAINT v8.30C, Madison, WI, USA, 2013.

[162] G. M. Sheldrick, XPREP in SHELXTL 2014/2, Madison, WI, USA, 2014.

[163] G. M. Sheldrick, Acta Cryst. A 2015, 71, 3-8.

[164] G. M. Sheldrick, SHELXL in SHELXTL v2014/7, Madison, WI, USA, 2014.

[165] C. B. Huebschle, G. M. Sheldrick, B. Dittrich, J. Appl. Cryst. 2011, 44, 1281-1284.

[166] (a) F. Hirshfeld, Acta Cryst. A 1976, 32, 239-244; (b) K. N. Trueblood, J. D. Dunitz, Acta Cryst. B 1983, 39, 120-133.

[167] G. M. Sheldrick, XP in SHELXTL v2014/7, Madison, WI, USA, 2014.

[168] M. Tretiakov, Y. G. Shermolovich, A. P. Singh, P. P. Samuel, H. W. Roesky, B. Niepötter, A. Visscher, D. Stalke, Dalton Trans. 2013, 42, 12940-12946.

[169] B. Milde, M. Leibeling, A. Hecht, P. G. Jones, A. Visscher, D. Stalke, J. Grunenberg, D. B. Werz, Chem.-Eur. J. 2015, 21, 16136-16146. 


\section{CURRICULUM VITAE}

Name: Arne Visscher

Anschrift: Kreuzbergring 68

37075 Göttingen

Email: avisscher@gwdg.de
Geburtsdatum: 03.02.1986

Geburtsort: Burgwedel

Familienstand: verheiratet

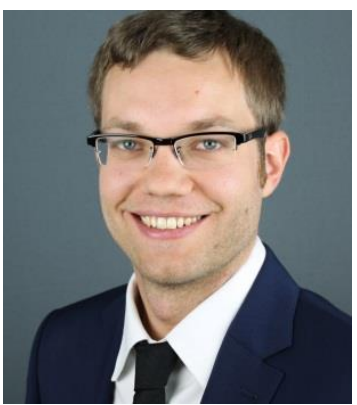

AKADEMISCHE AUSBILDUNG

$01 / 2011$ - heute

Promotion im Arbeitskreis von Prof. Dr. Dietmar Stalke, Institut für Anorganische Chemie, Georg-August-Universität Göttingen:

„Fluorescence Studies of Amine-substituted Azaanthracene Metal

Complexes"

$10 / 2009-11 / 2011$

Studium der Chemie an der Georg-August-Universität Göttingen,

Abschluss als M.Sc. mit der Masterarbeit ,Azaanthracene als

Chemosensoren" (Note: 1,0)

10/2006 - 08/2009 Studium der Chemie an der Georg-August-Universität Göttingen,

Abschluss als B.Sc. mit der Bachelorarbeit „Von lithiierten

Phenanthrenen zu Phosphanen" (Note: 1,0)

\section{ERFAHRUNG AUßERHALB UNIVERSITÄRER FORSCHUNG}

$01 / 2012-06 / 2015$

$10 / 2009-06 / 2015$

$11 / 2012-03 / 2014$

$03 / 2012-03 / 2014$

$10 / 2013$

$04 / 2011-03 / 2012$
Wissenschaftlicher Mitarbeiter am Institut für Anorganische Chemie, Georg-August-Universität Göttingen

Betreuung von Praktika und Durchführung von Seminaren für Studierende mit Haupt- oder Nebenfach Chemie

Gewählter Sprecher der Promovierenden im Vorstand von GAUSS (Georg-August University School of Science)

Gewählter Sprecher der Promovierenden der Fakultät für Chemie

Experimentelle Messungen an Röntgendiffraktometern, Firma Incoatec, Geesthacht

Planung, Projektleitung und Umsetzung des Lehrvideoprojekts „Virtuelles Labor" http://www.stalke.chemie.uni-goettingen.de/ virtuelles_labor/nmr/de.html 
ABITUR UND ZIVILDIENST

05/2006 - 07/2006 Angestellter bei der gGIS (gemeinnützige Gesellschaft für integrative Sozialdienste) in Hannover

08/2005 - 05/2006 Zivildienst bei der gGIS (gemeinnützige Gesellschaft für integrative Sozialdienste) in Hannover

08/1998 - 07/2005 Abitur am Gymnasium Isernhagen (Note: 2,1)

\section{ZUSATZQUALIFIKATIONEN UND AUSZEICHNUNGEN}

07/2015 Erhalt des Lehrpreises für die „, beste nicht-selbstständige Lehre im Institut für Anorganische Chemie"

$09 / 2013$

Seminarteilnahme „Interkulturelle Zusammenarbeit““

$12 / 2012$

Teilnahme am Hochschuldidaktikkurs „Meine Rolle als Lehrender“

$06 / 2012-05 / 2015$

Durchführung von Einkristall-Diffraktionsexperimenten von Service-

Partnern des Arbeitskreises

$06 / 2008$

Erhalt des Sachkundenachweises nach $§ 5$ ChemVerbotsV

\section{METHODEN UND FACHKENNTNISSE}

Analytik

Labor

\section{SPRACH- UND EDV-KENNTNISSE}

Sprachkenntnisse

EDV-Kenntnisse
Deutsch: Muttersprache

Englisch: fließend in Wort und Schrift

Französisch: Grundkenntnisse (A1)

sicherer Umgang mit MS Office

sicherer Umgang mit Chemie Programmen (ChemDraw, Origin, MestreNova, TopSpin)

sicherer Umgang mit kristallographischen Programmen (APEXII,

SHELX Suite, Mercury) 


\section{TEILNAHME AN KONFERENZEN}

9. Treffen des Center for Materials Crystallography, Åarhus (DK); Vortrag: "Sensing Metal Ions with Acridine Amines"

8. Treffen des Center for Materials Crystallography, Göttingen

$02 / 2014$ Central European Conference on Photochemistry, Bad Hofgastein (AU); Posterpräsentation: „New Acridinyl Amines as Potential Chemosensors"

6. Treffen des Center for Materials Crystallography, Göttingen Workshop der Firma Sartorius GmbH, Göttingen; Posterpräsentation: „Virtuelles Labor“"

$09 / 2012$ 16. Vortragstagung der Wöhler-Vereinigung, Göttingen; Posterpräsentation: „Virtuelles Labor“ 5. JungChemikerForum der GDCh, Göttingen; Posterpräsentation

$06 / 2011$

3. Treffen des Center for Materials Crystallography, Åarhus (DK)

$04 / 2010$

1. Treffen des Center for Materials Crystallography, Åarhus (DK)

\section{VERÖFFENTLICHUNGEN}

A. Visscher, S. Bachmann, C. Schnegelsberg, T. Teuteberg, R. Mata, D. Stalke „Highly selective and sensitive fluorescence detection of $\mathrm{Zn}^{2+}$ and $\mathrm{Cd}^{2+}$ ions by using an acridine sensor" in Dalton Trans. 2016, 45, 5689-5699.

N. Finkelmeier, A. Visscher, S. Wandtke, R. Herbst-Irmer, D. Stalke „Deprotonation of 9-Methylanthracene under Mild Conditions to give a Localized Carbanion" in Chem. Commun. 2016, $52,5440-5442$.

B. Milde, M. Leibeling, A. Hecht, P. G. Jones, A. Visscher, D. Stalke, J. Grunenberg, D. B. Werz „Oligoene-Based $\pi$-Helicenes or Dispiranes? Winding up Oligoyne Chains by a Multiple Carbopalladation/Stille/(Electrocyclization) Cascade" in Chem. Eur. J. 2015, 21, 16136-16146.

M. Tretiakov, Y. G. Shermolovich, A. P. Singh, P. P. Samuel, H. W. Roesky, B. Niepötter, A. Visscher, D. Stalke „Lewis-base stabilized diiodine adducts with $\mathrm{N}$-heterocyclic chalcogenamides" in Dalton Trans. 2013, 42, 12940-12946.

Göttingen, den 26.04.2016

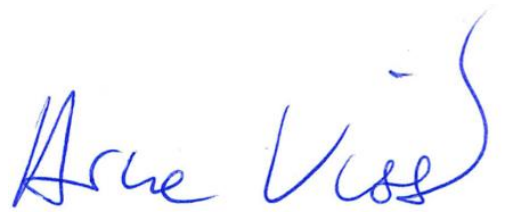


